Scenario Generation and Reduction for Long-term and Short-term Power System Generation Planning under Uncertainties

\author{
by \\ Yonghan Feng
}

A dissertation submitted to the graduate faculty

in partial fulfillment of the requirements for the degree of

DOCTOR OF PHILOSOPHY

Major: Industrial Engineering

Program of Study Committee:

Sarah M. Ryan, Major Professor

James D. McCalley

William Q. Meeker

Jo Min

Lizhi Wang

Iowa State University

Ames, Iowa

2014

Copyright (c) Yonghan Feng, 2014. All rights reserved. 


\section{DEDICATION}

To my beloved family. Thank you for your love, care, enlightenment and inspiration through my life. Thank you for your always encouraging and supporting at any time.

To all my dearest friends. Thank you for your guidance and encouragement through my graduate study. I appreciate those precious moments we spent together. 


\section{TABLE OF CONTENTS}

LIST OF TABLES $\ldots \ldots \ldots \ldots \ldots \ldots \ldots \ldots \ldots \ldots \ldots \ldots$

LIST OF FIGURES $\ldots \ldots \ldots \ldots \ldots \ldots \ldots \ldots \ldots \ldots \ldots$ viii

ACKNOWLEDGEMENTS $\ldots \ldots \ldots \ldots \ldots \ldots \ldots \ldots \ldots \ldots \ldots \ldots \ldots \ldots \ldots \ldots \ldots \ldots$

ABSTRACT $\ldots \ldots \ldots \ldots \ldots \ldots \ldots \ldots \ldots \ldots \ldots \ldots \ldots \ldots \ldots \ldots \ldots \ldots \ldots \ldots \ldots \ldots$

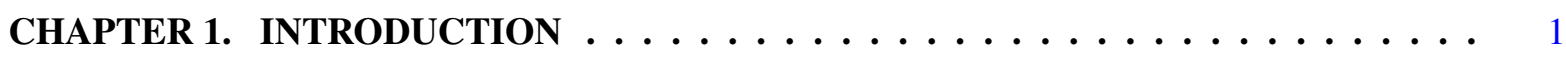

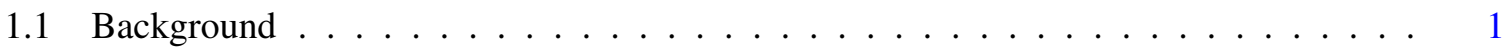

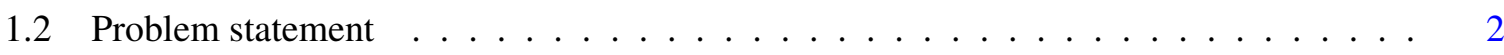

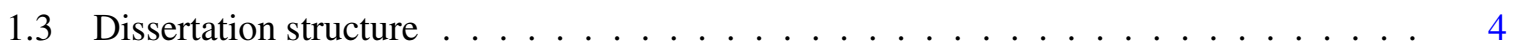

CHAPTER 2. SCENARIO CONSTRUCTION AND REDUCTION APPLIED TO STOCHAS-

TIC POWER GENERATION EXPANSION PLANNING . . . . . . . . . . . . 6

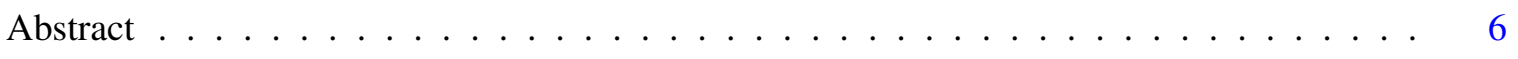

2.1 Introduction $\ldots \ldots \ldots \ldots \ldots \ldots \ldots \ldots \ldots \ldots \ldots \ldots \ldots \ldots \ldots \ldots \ldots \ldots \ldots$

2.2 Related literature . . . . . . . . . . . . . . . . . . . . . 9

2.3 Generation expansion planning model $\ldots \ldots \ldots \ldots \ldots \ldots$

2.4 Scenario generation . . . . . . . . . . . . . . . . . . 14

2.4.1 Optimization model for scenario tree generation . . . . . . . . . . 15

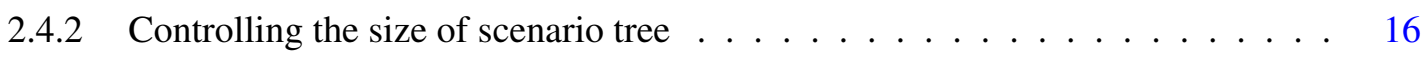

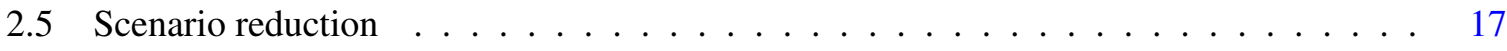

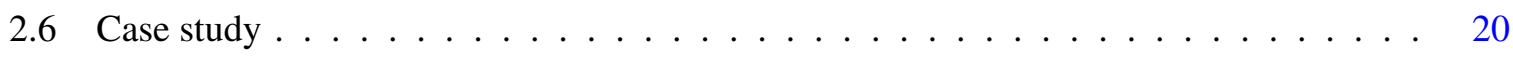

2.6.1 Geometric Brownian motion assumption and verification . . . . . . . . . 21

2.6.2 Important properties of GBM for scenario generation . . . . . . . . . . 22

2.6.3 Division of the planning horizon and scenario tree generation . . . . . . . . 24 


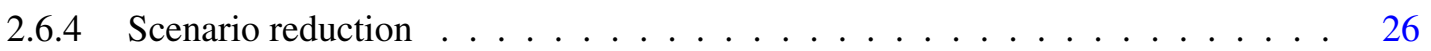

2.6 .5 Numerical results . . . . . . . . . . . . . . . . . . . . . 30

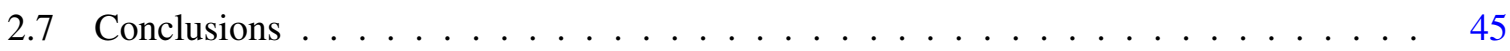

CHAPTER 3. DAY-AHEAD HOURLY ELECTRICITY LOAD MODELING BY FUNC-

TIONAL REGRESSION $\ldots \ldots \ldots \ldots \ldots \ldots \ldots$

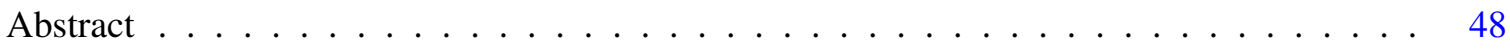

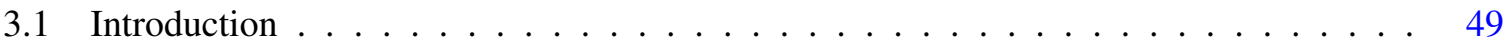

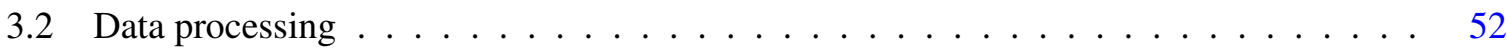

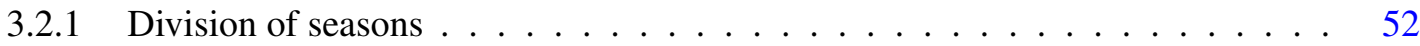

3.2 .2 Days segmentation . . . . . . . . . . . . . . . 53

3.2 .3 Load data transformation . . . . . . . . . . . . . . . . 54

3.3 Epi-spline based day-ahead hourly models . . . . . . . . . . . . . . . 55

3.3.1 Approximation by epi-spline . . . . . . . . . . . . . . . 57

3.3.2 Weather conditional epi-spline (WEp) load model . . . . . . . . . . . . . . 57

3.3.3 Fundamental load incorporated weather conditional epi-spline (FWEp) load model ............................ 59

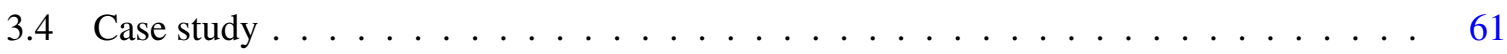

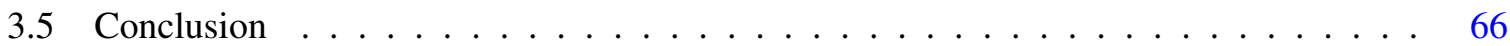

3.6 Remark: Scenario generation . . . . . . . . . . . . . . . . . 72

3.6.1 Density estimation of prediction errors . . . . . . . . . . . . . 72

3.6.2 Probabilistic load scenario generation . . . . . . . . . . . . . 74

3.7 Appendix A: Derivation of one-dimension epi-spline formulation . . . . . . . . . . 76

3.8 Appendix B: Some comments on high-dimension and multi-variable epi-spline con-

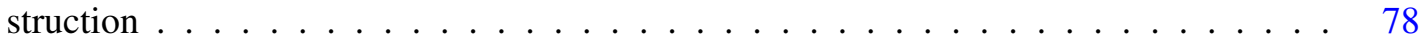

3.8.1 High-dimension and multi-variable epi-splines $\ldots \ldots \ldots \ldots$

3.8.2 Theory of constructing high-dimension and multi-variables epi-splines $\ldots . .80$

CHAPTER 4. SOLUTION SENSITIVITY-BASED SCENARIO REDUCTION FOR S-

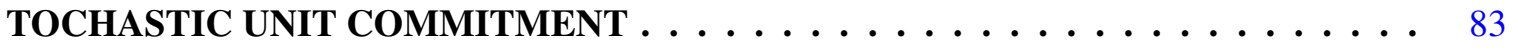




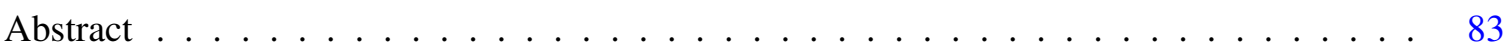

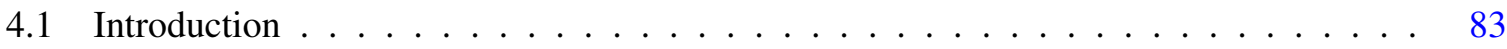

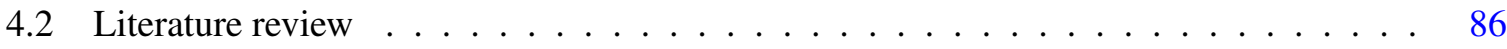

4.3 Two-stage stochastic reliability unit commitment model . . . . . . . . . . . . . . . 88

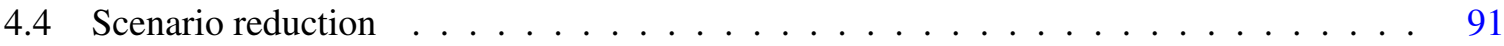

4.4.1 Forward selection in recourse clusters $($ FSRC $) \quad \ldots \ldots \ldots$. . . . . . . . 92

4.4 .2 Customization strategies of FSRC $\ldots \ldots \ldots \ldots$

4.4.3 Evaluation of selected scenarios . . . . . . . . . . . . . . . . . 98

4.5 Case studies . . . . . . . . . . . . . . . . . . . . . . . 100

4.5.1 Scenario generation . . . . . . . . . . . . . . 100

4.5.2 Independent daily SRUC . . . . . . . . . . . . . . . . . . . . . . 101

4.5.3 Rolling horizon SRUC over a week . . . . . . . . . . . . . . . . 107

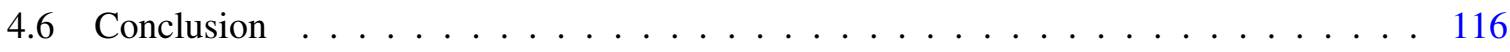

4.7 Remark: Extensions of FSRC . . . . . . . . . . . . . . . . . . . . . 117

4.8 Appendix: Concrete stochastic reliability unit commitment model f . . . . . . . . 122

4.8 .1 Notation . . . . . . . . . . . . . . . . . . 122

4.8.2 Mathematical model . . . . . . . . . . . . . . . . . . 123

CHAPTER 5. GENERAL CONCLUSIONS $\ldots \ldots \ldots \ldots \ldots \ldots \ldots$

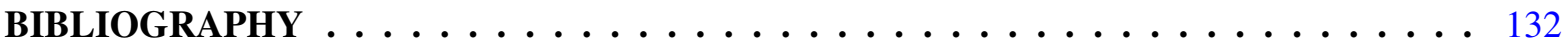




\section{LIST OF TABLES}

Table 2.1 The parameters for candidate generators \& existing generation capacity _ . . 21

Table 2.2 Statistical properties of log-ratios of annual electricity demand and natural gas

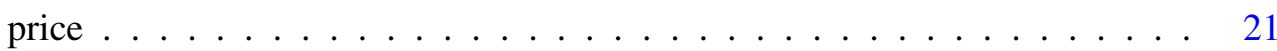

Table 2.3 The growth rates of annual electricity demand and natural gas price for periods

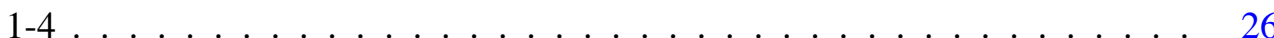

Table 2.4 The growth rates of annual electricity demand and natural gas price for periods

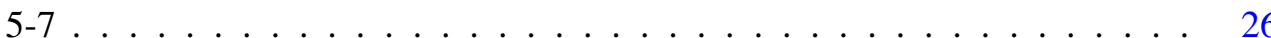

Table 2.5 The growth rates of annual electricity demand and natural gas price for periods

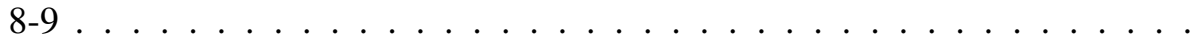

Table 2.6 The growth rates of annual electricity demand and natural gas price for period

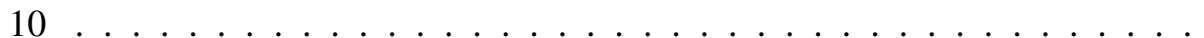

Table 2.7 The growth rates of annual electricity demand and natural gas price for even years in the second scenario tree $\ldots \ldots \ldots \ldots \ldots$

Table 2.8 The cumulative number of generators added based on FFS reduction of the unequal-length period tree $\ldots \ldots \ldots . \ldots . \ldots 34$

Table 2.9 The cumulative number of generators added based on FSWC reduction of the unequal-length period tree $\ldots \ldots \ldots \ldots$. . . . . . . . . . 34

Table 2.10 Expected costs and solution times based on FFS \& FSWC for the unequal-

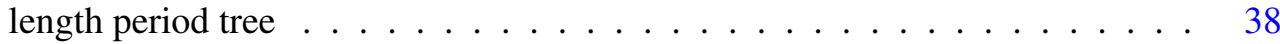

Table 2.11 The cumulative number of generators added based on FFS reduction of the equal-length period tree . . . . . . . . . . . . . . . . 39

Table 2.12 The cumulative number of generators added based on FSWC reduction of the equal-length period tree $\ldots \ldots \ldots \ldots$. . . . . . . . . . . . . 
Table 2.13 Expected costs and solution times based on FFS \& FSWC for the equal-length period tree ......................... 46

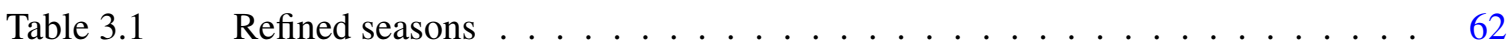

Table 3.2 MAPEs of prediction errors of WEp, FWEp and AL in Apr. 2011 - Mar. 2012

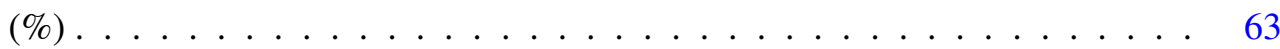

Table 3.3 MAPEs of prediction errors of FWEp, Hong and C\&S in Apr. 2011 - Mar.

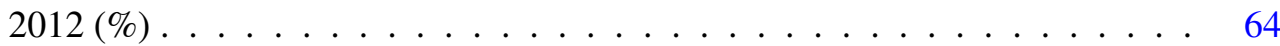

Table 3.4 RMSEs of prediction errors of FWEp and other two benchmark models in Apr. 2011 - Mar. $2012(\mathrm{MW}) \ldots \ldots \ldots$. . . . . . . . . . . . . 64

Table 4.1 Expected values of the all-scenarios based SRUC through a week, 2011-07-10 to $2011-07-16, n=80 \ldots \ldots \ldots \ldots \ldots \ldots$

Table 4.2 Expected values of UC from TC with respect to all scenarios through a week, 2011-07-10 to 2011-07-16, $n=40 \ldots \ldots \ldots \ldots \ldots \ldots \ldots \ldots \ldots$

Table 4.3 Expected values of UC from TC+PC with respect to all scenarios through a week, 2011-07-10 to 2011-07-16, $n=40 \ldots \ldots \ldots \ldots \ldots$. . . . . . . . . 


\section{LIST OF FIGURES}

Figure 2.1 Sample ACF of log growth ratios: (up) annual electricity demand, (down)

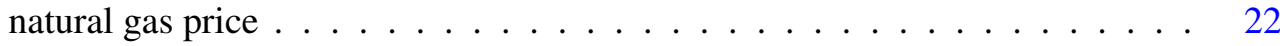

Figure 2.2 Division of the planning horizon time into increasing length periods . . . . 25

Figure 2.3 Division of the planning horizon time into equal length periods . . . . . . . . 25

Figure 2.4 Scenario tree with unequal length periods: (up) demand vs. time, (down) gas

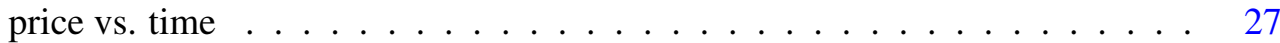

Figure 2.5 Scenario lattice with equal length periods: (up) demand vs. time, (down) gas

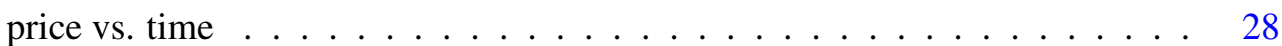

Figure 2.6 Scenarios selected by FFS method from the unequal-length scenario set (tree):

(up) demand vs. time, (down) gas price vs. time . . . . . . . . . . . . . 31

Figure 2.7 Scenarios selected by FSWC method from the unequal-length scenario set (tree): (up) demand vs. time, (down) gas price vs. time $\ldots \ldots . . \ldots 32$

Figure 2.8 Comparisons among scenarios from original set, FSWC and FFS methods based on the unequal-length scenario set (tree): (up) expected annual electricity demand, (down) expected average natural gas price . . . . . . . . . . 33

Figure 2.9 Illustration of expected energy generation of each type of generator based on the unequal-length period tree: (up) FFS method, (down) FSWC method . . . 35

Figure 2.10 Expected generation cost of each type of generator over selected scenarios from the unequal-length period tree: (up) FFS method, (down) FSWC method 36

Figure 2.11 Scenarios selected by FFS method from the equal-length period lattice: (up) demand vs. time, (down) gas price vs. time . . . . . . . . . . . . 40

Figure 2.12 Scenarios selected by FSWC method from the equal-length period lattice: (up) demand vs. time, (down) gas price vs. time . . . . . . . . . . . . . . 41 
Figure 2.13 Comparison of average electricity demand and natural gas price between two methods based on the equal-length period lattice: (up) expected annual electricity demand, (down) expected average natural gas price . . . . . . . . . . 42

Figure 2.14 Illustration of expected energy generation of each type of generator based on the equal-length period lattice: (up) FFS method, (down) FSWC method . . .

Figure 2.15 Expected generation cost of each type of generator over selected scenarios from the equal-length period lattice: (up) FFS method, (down) FSWC method

Figure 3.1 Scatter plot of load vs. temperature for each month in the training set, CT (Apr. 2009 - Mar. 2011) . . . . . . . . . . . . . . . . 53

Figure 3.2 24-hours temperature through summers in the training set, CT (Apr. 2009 -

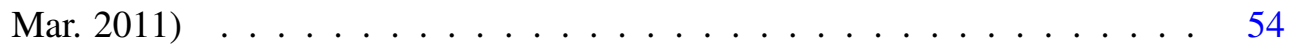

Figure 3.3 Average daily load sequence in each day type in Apr. 2009 - Mar. 2011, CT . 56

Figure 3.4 Load vs. dew point temperature forecast for hours within specified range of temperature forecast in summer, $\mathrm{CT} \ldots \ldots \ldots 58$

Figure 3.5 Average hourly temperature vs. average hourly load through a day in Apr. 2009 - Mar. 2011, CT . . . . . . . . . . . . . . . . . . 60

Figure 3.6 Box plots of prediction errors in Apr. 2011 - Mar. 2012 for FWEp and other two benchmark models: spring . . . . . . . . . . . . . . . . . 67

Figure 3.7 Box plots of prediction errors in Apr. 2011 - Mar. 2012 for FWEp and other two benchmark models: summer . . . . . . . . . . . . . . . . . . . . 68

Figure 3.8 Box plots of prediction errors in Apr. 2011 - Mar. 2012 for FWEp and other two benchmark models: fall . . . . . . . . . . . . . . . . . . . . . . . 69

Figure 3.9 Box plots of prediction errors in Apr. 2011 - Mar. 2012 for FWEp and other two benchmark models: winter . . . . . . . . . . . . . . . . . 70

Figure 3.10 Box plot of prediction errors of FWEp and two benchmark models for ISO-NE in test set (Apr. 2011 - Mar. 2012) _ . . . . . . . . . . . . . 71

Figure 3.11 InACF of actual and forecast daily load trajectory of ISO-NE in test set (Apr. 2011 - Mar. 2012) . . . . . . . . . . . . . . . . 71 
Figure 3.12 Average absolute errors in daily load trajectory of ISO-NE in test set (Apr. 2011 - Mar. 2012) . . . . . . . . . . . . . . . . 72

Figure $4.1 \quad$ Scenario reduction procedure of customized FSRC method . . . . . . . . . 98

Figure $4.2 \quad 80$ net load scenarios on $2011-07-11 \ldots \ldots \ldots \ldots$. . . . . . . . . 102

Figure 4.320 selected scenarios by FFS on 2011-07-11 . . . . . . . . . . . . . . 103

Figure 4.420 selected scenarios by FSRC: TC on 2011-07-11 . . . . . . . . . . . . . . 104

Figure 4.5 Deviations from optimal commitment and generation cost of FSRC and FFS through the summer week, 2011-07-10 to 2011-07-16, for different cardinality

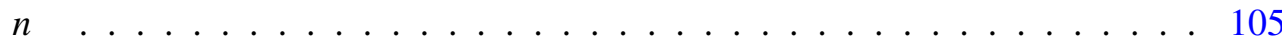

Figure 4.6 Deviation from optimal load imbalance of FSRC and FFS through the summer week, 2011-07-10 to 2011-07-16, for different cardinality $n$. . . . . . . . 106

Figure 4.720 Selected scenarios by FSRC: TC+PC on 2011-07-11 _ . . . . . . . . 107

Figure 4.8 Savings in commitment and generation cost of customized FSRC methods from FFS in evaluation, $n=20 \ldots \ldots \ldots \ldots$

Figure 4.9 Savings in shortage of FSRC from FFS in evaluations, $n=20 \ldots \ldots$

Figure 4.10 Savings in excess of FSRC from FFS in evaluations, $n=20 \ldots \ldots$. . . . . 109

Figure 4.11 Time spent on scenario clustering and selection by FSRC for different number$\mathrm{s}, n$, of scenarios selected from 1350 . The bar marker in each box represents the median and the round marker denotes the mean time over 12 randomly selected days in $2011 \ldots \ldots \ldots$. . . . . . . . . . . . . . . . 110

Figure 4.12 Expected savings in load imbalance of FSRC methods from FFS for 20 selected scenarios through different pairs of penalties for the week 2011-07-10 to

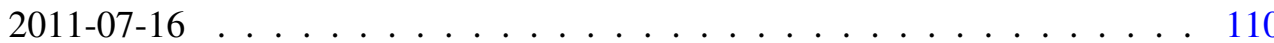

Figure 4.13 Relative differences in hourly capacity committed between half-scenarios based rolling horizon SRUC and all-scenarios based rolling horizon SRUC in week

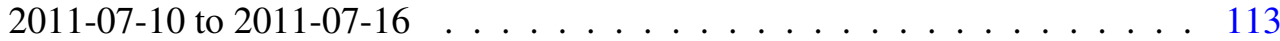

Figure $4.14 \quad \mathscr{Z}\left(\mathscr{S}_{F S R C: T C}^{\prime}\right)$ vs. $\mathscr{Z}\left(\mathscr{S}_{F S R C: T C+P C}^{\prime}\right)$ for different cardinality $n$ in week 201107-10 to 2011-07-16 . . . . . . . . . . . . . . . . . . . . 114 
Figure 4.15 Relative differences in expected load imbalance for different cardinalities, $n$, through the week 2011-07-10 to 2011-07-16: (a) $\mathscr{W}^{+}\left(\mathscr{S} \mid \mathscr{S}_{F S R C: T C}^{\prime}\right)$ vs. $\mathscr{W}^{+}\left(\mathscr{S} \mid \mathscr{S}_{F S R C: T C+P C}^{\prime}\right),(\mathrm{b}) \mathscr{W}^{-}\left(\mathscr{S} \mid \mathscr{S}_{F S R C: T C}^{\prime}\right)$ vs. $\mathscr{W}^{-}\left(\mathscr{S} \mid \mathscr{S}_{F S R C: T C+P C}^{\prime}\right) \ldots 114$

Figure $4.16 \mathscr{Z}\left(\mathscr{S}_{F S R C: T C}^{\prime}\right)$ vs. $\mathscr{Z}\left(\mathscr{S}_{F S R C: T C+P C}^{\prime}\right)$ over different penalty settings in week 2011-07-10 to 2011-07-16 . . . . . . . . . . . . . . . 115

Figure 4.17 Relative differences in load imbalances through different penalty settings:

(a) $\mathscr{W}^{+}\left(\mathscr{S} \mid \mathscr{S}_{F S R C: T C}^{\prime}\right)$ vs. $\mathscr{W}^{+}\left(\mathscr{S} \mid \mathscr{S}_{F S R C: T C+P C}^{\prime}\right)$

(b) $\mathscr{W}^{-}\left(\mathscr{S} \mid \mathscr{S}_{F S R C: T C}^{\prime}\right)$ vs. $\mathscr{W}^{-}\left(\mathscr{S} \mid \mathscr{S}_{F S R C: T C+P C}^{\prime}\right) \ldots \ldots \ldots$

Figure 4.18 Relative differences from the optimal commitment cost . . . . . . . . . . 118

Figure 4.19 Relative differences from the optimal expected generation cost . . . . . . . . 119

Figure 4.20 Relative differences from the optimal commitment and generation cost . . . . 119

Figure 4.21 Comparisons to the optimal shortage $\ldots \ldots \ldots \ldots \ldots$

Figure 4.22 Comparisons to the optimal excess f . . . . . . . . . . . . 120

Figure 4.23 Comparisons on total scenario time . . . . . . . . . . . . . . . . . 121 


\section{ACKNOWLEDGEMENTS}

I would like to take this great opportunity to express my gratitude to those who helped me with various aspects of my graduate study, academic research, and the writing of this dissertation.

First and foremost, I sincerely thank my major professor, Dr. Sarah Ryan, for her excellent guidance, patience and constant support throughout my graduate study and research. Her rich insights and rigorous scholarship have inspired me to pursue a higher academic goal. Without her patience and unlimited support, I would not have been able to complete this research and writing of this dissertation. I would like to thank my committee members for their efforts and contributions to this work: Dr. James McCalley, Dr. William Meeker, Dr. Jo Min and Dr. Lizhi Wang. I am also greatly thankful to my co-authors, Dr. Jean-Paul Watson at Sandia National Laboratory, Dr. David Woodruff and Dr. Roger Wets at the University of California Davis, from whom I gained enormous knowledge. I would also like to thank Dr. John Birge at the University of Chicago and Dr. Audun Botterud in the Decision and Information Sciences Division of Argonne National Laboratory for their consistent encouragement and supporting during my internship, and opening another window for my academic research work.

I greatly acknowledge the Power Systems Engineering Research Center (PSERC) and Advanced Research Projects Agency (ARPA-E) - Energy, U.S. Department of Energy for their sponsorship of my research. 


\begin{abstract}
This dissertation focuses on computational issues of applying two-stage stochastic programming for long-term and short-term generation planning problems from the perspective of scenario generation and reduction. It follows a three-paper format, in which each paper discusses approaches to generating probabilistic scenarios and then reducing the substantial computational burden caused by a huge number of scenarios for different applications in power systems.

The first paper investigates a long-term generation expansion planning model with uncertain annual load and natural gas price. A two-stage stochastic program is formulated to minimize the total expected expansion cost, generation cost and penalties on unserved energy while satisfying aggregated operational constraints. A statistical property matching technique is applied to simulate plausible future realizations of annual load and natural gas price over the whole planning horizon. To mitigate the computational complexity of a widely used classic scenario reduction method in this context, we firstly cluster scenarios according to the wait-and-see solution for each scenario and then apply the fast forward selection (FFS) method.

The second paper prepares a basis for load scenario generation for the day-ahead reliability unit commitment problem. For the purpose of creating practical load scenarios, epi-splines, based on approximation theory, are employed to approximate the relationship between load and weather forecasts. The epi-spline based short-term load model starts by classifying similar days according to daily forecast temperature as well as monthly and daily load patterns. Parameters of the epi-spline based short-term load model are then estimated by minimizing the fitted errors. The method is tested using day-ahead weather forecast and hourly load data obtained from an Independent System Operator in the U.S. By considering the non-weather dependent load pattern in the short-term load model, the model not only provides accurate load predictions and smaller prediction variances in the validated days, but also preserves similar intraday serial correlations among hourly forecast loads to those from actual load.

The last paper in this dissertation proposes a solution-sensitivity based heuristic scenario reduction
\end{abstract}


method, called forward selection in recourse clusters (FSRC), for a two-stage stochastic day-ahead reliability unit commitment model. FSRC alleviates the computational burden of solving the stochastic program by selecting scenarios based on their cost and reliability impacts. In addition, the variant of pre-categorizing scenarios improves the computational efficiency of FSRC by simplifying the clustering procedure. In a case study down-sampled from an Independent System Operator in the U.S., FSRC is shown to provide reliable commitment strategies and preserve solution quality even when the reduction is substantial. 


\section{CHAPTER 1. INTRODUCTION}

\subsection{Background}

As one of the major energy sources, electricity has dramatically changed people's daily lives, and rapidly improved human society. With the increasing growth of economy and production activities, the electricity usage has risen substantially in today's modern life. According to the estimation of the U.S. Energy Information Administration (EIA), nearly 4 trillion KWh of electricity was consumed in 2013 in the U.S., about 13 times greater than the electricity usage in 1950 (U.S. Energy Information Administration, 2012b, 2013a). However, the upward growth in electricity demand also leads to more challenging issues in operating modern power systems, such as extending generation capacity, security issues of daily power generation and cost of power generation. All of these issues have led major electricity consuming sectors to call for a more reliable, safe and economic power supply.

Fluctuations among electricity demand levels over time and intermittence of variable generation are two major factors making reliable and economical generation more challenging than ever before. From the statistical information of EIA, monthly electricity consumption level has fluctuated dramatically since 2000 (U.S. Energy Information Administration, 2013b). Many research papers have revealed that the electricity demand fluctuation is caused by alternations of seasons, changes of weather conditions, and human activities. However, it is still an ongoing work to learn exactly how these factors drive electricity consumption, as well as to identify other contributing factors. Because of these uncertainties, it is inevitable to have prediction errors in electricity demand forecasts for the next years, days or even hours. Therefore, it should not be a surprise that the inaccurate electricity predictions cause more actions to avoid load imbalance, and eventually result in higher operational cost. To achieve more reliable and economical generation plans, many efforts have been taken to improve the accuracy of electricity demand forecasting and create a more flexible generation schedule to handle uncertain demand. 
With the increasing growth of concerns about climate changes, new power generation technologies have been developed in past decades, which impose remarkable changes in modern power systems. Many countries and U.S. states have established their own standards for renewable energy penetration in power systems. The European Union set a Renewable Portfolio Standard (RPS) goal for 25\% share of power generation by 2030 (European Federation of Energy Traders, 2013). Most states in the U.S. also set their own RPS goals. Some of them even set aggressive goals for the share of renewable energy to equal $25 \%$ or $30 \%$ of total electricity by 2030 (Jaccard, 2004). Over the past years, electricity generation provided by renewable energy has substantially increased: the share of U.S. electricity generation from renewable generation grew to $13 \%$ in 2013, nearly doubled over ten years (U.S. Energy Information Administration, 2013a,c). Among these resources, the largest share, about 53\%, of the renewable electricity came from hydroelectric power, followed by wind (32\%), solar and biomass (U.S. Energy Information Administration, 2013c). However, the unpredictable intermittence of renewable energy, i.e. wind energy and solar energy, leads to more challenges to maintaining reliability. For instance, many ancillary services and prevention actions are required if the planned energy is not delivered successfully. Therefore, it is an urgent research work to discuss how to flexibly schedule the on/off status of thermal generators with penetration of renewable and variable generation to keep power systems reliable, while lowering the operational cost.

\subsection{Problem statement}

Many of the existing papers have addressed power generation with variable resources by adopting scenario based two-stage stochastic programming, and obtained promising numerical results and conclusions. However, most of these papers mainly focus on the modeling and algorithm design for the stochastic program, but only slightly touch on the topic of practical scenario generation and computation of power system generation planning with a large set of scenarios. Therefore, how to properly create a batch of practical scenarios and then maintain a tractable computation burden rises as another significant issue in applying stochastic programming to power systems.

This dissertation address several modeling and computational issues related to long-term generation expansion planning and day-ahead short-term generation planning of power systems using stochastic 
programming.

The dissertation begins with a long-term generation expansion planning model that assumes a central regulator targeting a system-wide cost minimization decision on how many units of what type of power generators to build in which year and how much electricity should be generated by each type of generator. Because the uncertainties through the long-term planning horizon impose substantial influences on investment and operational decisions, the first paper addresses the following issues:

1. Modeling an integrated multi-period generation expansion planning problem including multiple uncertain resources;

2. Modeling the evolutions of uncertain resources through a long-term planning horizon;

3. Achieving an acceptable tradeoff between computational complexity and solution accuracy when there are a large number of possible realizations of uncertain parameters in the generation expansion planning problem.

Compared to the long-term generation expansion planning problem, more concrete and sophisticated operational constraints need to be considered in a short-term generation planning problem with uncertainties, i.e. the stochastic reliability unit commitment (SRUC) problem. Uncertain factors, such as electricity demand and variable generation, will influence the commitment strategy and generation levels of all thermal generators. Therefore, the second paper prepares a day-ahead hourly load model as the prerequisite of generating possible and reasonable hourly electricity trajectories for SRUC, including:

1. An approach to modeling the complicated nonlinear relationship between hourly electricity demand and weather forecast, and predicting hourly load based on the day-ahead weather forecast;

2. Investigations on how similar the trajectory of predicted hourly electricity demand through a day is to the actual one.

Once the probabilistic scenarios of hourly load and variable generation are created, the day-ahead SRUC can be solved. However, if there are many scenarios input for SRUC, the optimization problem solving procedure can seldom be finished in a manageable time. Therefore, the third paper considers the following computational issues: 
1. Selecting a modest number of scenarios which follow decision maker's concerns on cost and reliability, as well as lead to a solution that is similar to the one from the whole set of scenarios;

2. Efficiently measuring impacts of scenarios on decisions, and improving the computational efficiency of the selection procedure;

3. Evaluating the quality of selected subset of scenarios.

\subsection{Dissertation structure}

This dissertation consists of three papers. The first paper, published in Computers \& Operations Research, is presented in Chapter 2. This paper identifies the numbers and building years of candidate technologies of generators through the planning horizon of a long-term generation expansion problem, while minimizing total expansion cost, expected generation cost and expected risk (measured by expected penalties on unserved energy). The major uncertainties of the generation expansion planning problem are assumed to be annual electricity demand and natural gas price throughout the whole planning period. Because the expansion strategies directly depend on the input scenarios, this paper first presents a way to generate annual electricity and price scenarios simultaneously by applying a statistical properties matching technique. However, given the huge set of multi-year scenarios generated, the exponentially increased scale of the extensive form of the two-stage stochastic program makes its solution procedure intractable. Instead of obtaining the optimal expansion strategy from the whole set of scenarios, the latter part of the first paper applies a heuristic scenario reduction method that accounts for the impacts from scenario parameters on first-stage solutions. In addition, because the widely used classical scenario reduction methods, forward selection and fast forward selection, still lack practical efficiency when a modest number of scenarios are to be selected from a huge set of scenarios, the first paper also targets on computational time savings.

The second paper, in preparation for submission to Applied Energy, appears in Chapter 3. This paper develops a tool that can approximate the features of the future possible hourly load realizations. Because the commonly used parametric regression technique either lacks flexibility to capture the load curve, or needs more improved prediction accuracy when there is a little longer prediction period, an approximation theory-based curve construction technique, called epi-spline approximation, is applied 
to model day-ahead short-term electricity demand. Prediction errors and intraday serial correlations of forecast hourly load that result from the proposed epi-spline based load model are also investigated to examine its suitability for generating load scenarios.

The last paper, published in Computational Management Science, is included in Chapter 4. It discusses a heuristic scenario reduction framework and two corresponding customizations for unit commitment. The framework reveals relative similarities among many scenarios by applying a baseline unit commitment to all scenarios, and tracks decision makers' concerns; namely, cost and reliability for operating power systems. The reliability is measured by the absence of shortage (curtailment in demand) and excess (curtailment in generation). One of the customizations focuses on reducing the complexity of assessing impacts of scenarios, while the other aims to cluster scenarios faster. The quality of a selected subset of scenarios is evaluated by solving SRUC based on the selected subsets, and then comparing commitment costs, expected shortage and excess to their optimal counterparts from the whole set of scenarios. Groups of case studies demonstrate that the proposed heuristic scenario reduction approach not only preserves solution quality and reliability from the perspective of less load imbalance, but also saves computational time.

Chapter 5 generally concludes the dissertation, and briefly introduces possible future research work. 


\title{
CHAPTER 2. SCENARIO CONSTRUCTION AND REDUCTION APPLIED TO STOCHASTIC POWER GENERATION EXPANSION PLANNING
}

\author{
A paper published in Computers \& Operations Research
}

Yonghan Feng and Sarah M. Ryan

\begin{abstract}
A challenging aspect of applying stochastic programming in a dynamic setting is to construct a set of discrete scenarios that well represents multivariate stochastic processes for uncertain parameters. Often this is done by generating a scenario tree using a statistical procedure and then reducing its size while maintaining its statistical properties. In this paper, we test a new scenario reduction heuristic in the context of long-term power generation expansion planning. We generate two different sets of scenarios for future electricity demands and fuel prices by statistical extrapolation of long-term historical trends. The cardinality of the first set is controlled by employing increasing length time periods in a tree structure while that of the second set is limited by its lattice structure with periods of equal length. Nevertheless, some method of scenario thinning is necessary to achieve manageable solution times. To mitigate the computational complexity of the widely-used forward selection heuristic for scenario reduction, we customize a new heuristic scenario reduction method named forward selection in wait-and-see clusters (FSWC) for this application. In this method, we first cluster the scenarios based on their wait-and-see solutions and then apply fast forward selection within clusters. Numerical results for a twenty year generation expansion planning case study indicate substantial computational savings to achieve similar solutions as those obtained by forward selection alone.
\end{abstract}




\subsection{Introduction}

A stochastic program is a mathematical program that involves some uncertain data. These parameters may be most accurately described as continuous random variables but, except in some trivial situations, it is difficult to optimize directly in terms of those distributions. Hence, in most applications, the continuous distributions are approximated by discrete distributions with a finite number of scenarios, or possible realizations, for the random variables. This discretization procedure is often called scenario generation. The stochastic programming model then can be specified by combining a deterministic formulation of the mathematical program, which could be solved for any scenario, with a set of scenarios for the uncertain data. Thus, a crucial step in the application of stochastic programming is to obtain a set of scenarios that realistically represents the distributions of the random parameters but is not too large.

Uncertainty is inescapable in long-term capacity planning applications. The random parameters can be conceived to follow a multidimensional stochastic process over the planning horizon, so that discrete scenarios represent sample paths. Even a moderate dimension for the process, when combined with a reasonable number of time steps in the planning horizon, can result in a very large number of such paths. In a two-stage stochastic programming formulation, the first-stage variables represent "here-and-now" decisions, such as facility investments, that must be taken before the realization of the uncertain data is known. The second stage,"wait-and-see" decisions represent system operation, within the constraints dictated by the investment decisions, as the random parameters evolve over time. In the extensive form of a two-stage stochastic program, a separate set of second stage decision variables is used for each possible scenario path and the problem can be solved as a (very high dimensional) deterministic mathematical program. Assuming scenario independence, the constraint coefficients form a large block-diagonal matrix in which each block describes the same constraint structure with scenariospecific parameters. Because of this special structure, several decomposition methods have been used to solve the two-stage model (Kall and Wallace, 1994). However, a large number of scenarios may limit the tractability of solution, even when using decomposition techniques. To attain reasonable computation times, it is natural to try to approximate the original scenario set with a much smaller subset that retains essential features, a process often called scenario reduction. 
Power generation expansion planning (GEP) is a complex multiple-year problem that aims to determine the timing and technology choices for generation investments over a long planning horizon under economic criteria while ensuring that expected electricity demand growth is met (Gonen, 1988). The traditional GEP involved solving centralized planning problems to identify plans that would minimize costs for the whole system even under adverse conditions. The typical objective has been to minimize the expected sum of discounted costs, including investment cost for generating units, operation and maintenance costs, and penalties for unserved energy (Gorenstin et al., 1993; Kagiannas et al., 2004; Park et al., 1999, 2000); more recent models have also considered risk (Jin et al., 2011). Recently, the electricity industry has undergone significant restructuring towards deregulation and competition, and attention has shifted to maximizing profits, which are composed of the revenues based on market prices and the costs, including both investment and operation, of individual generating companies in the market. However, restructuring has not yet taken hold in all regions of the US or in many parts of the world. The centralized GEP problem remains relevant, though it must increasingly account for growing uncertainties in fuel prices, availability of intermittent forms of renewable generation, possible environmental regulations, and demand stemming from increasing electrification (for example, of transportation) and implementation of smart grid technologies that increase consumer choice.

This paper investigates the application of a recently-developed scenario reduction heuristic, termed forward selection in wait-and-see clusters (FSWC) (Wang, 2010) to a two-stage stochastic programming formulation of GEP with uncertainty in demand growth and fuel prices. We generate scenarios for a case study by fitting a stochastic process model to historical data for electric load and natural gas price in the Midwest region of the US and construct scenario sets in the form of a tree or a lattice by a momentmatching method (Høyland and Wallace, 2001). The FSWC heuristic first solves a deterministic "waitand-see" subproblem for each scenario, in which both first- and second-stage decisions are chosen to minimize cost in that particular scenario. Then scenarios are clustered based on similarity of their "key" first-stage decisions. Identifying these key decisions is the application-specific aspect of FSWC. Finally, it applies the well-known forward selection heuristic (Dupačová et al., 2003; Heitsch and Römisch, 2003) to choose one representative scenario within each cluster. In our GEP case study with a 20 year planning horizon, the overall computational time required by applying FSWC can be up to two orders of magnitude smaller than that required by applying classical forward selection, depending on 
the cardinality of the reduced scenario set, while similar first-stage solutions are obtained

The contributions of this paper include statistically verifying that historical annual electricity demands and natural gas prices in the US have been consistent with correlated geometric Brownian motion (GBM) processes, and then generating scenario trees to extrapolate those processes by solving nonlinear programs derived from the moment matching method (Høyland and Wallace, 2001) under two methods of subdividing the planning horizon. In addition, the customization of FSWC for this application is described and tested.

The chapter is organized as follows. In Section 2.2 we place our research in context of the literature. Section 2.3 presents a simplified long term centralized GEP model. Section 2.4 reviews the general moment matching method for scenario tree generation. Section 2.5 describes a novel heuristic scenario reduction method based on wait-and-see clustering that applies the forward selection heuristic within clusters. For our case study, Section 2.6 first verifies the historical fit of correlated GBM processes for annual electricity demands and average natural gas prices in the U.S.. Then, a general scenario tree for unequal length sub-periods and a tree with lattice structure for equal-length sub-periods are generated for a twenty-year time horizon. Next, the FSWC heuristic is implemented for the two-stage stochastic programming model of GEP after defining the key first-stage variables. The related numerical results and comparisons between forward selection and FSWC are also provided. Finally, Section 2.7 provides conclusions.

\subsection{Related literature}

Many researchers have discussed approaches for generating appropriate scenarios (Kaut and Wallace, 2007). One of the most intuitive approaches is to sample directly from the specified marginal distribution and correlation matrix (Lurie and Goldberg, 1998). Another scenario generation method is based on optimal discretization. This method tries to approximate the stochastic process with finite scenarios by minimizing the pertinent error in the stochastic programming objective function of a discrete optimization model (Pflug, 2001). One of its advantages is to generate a whole multipleperiod scenario tree at once. In addition, scenarios can be generated by path-based methods. Instead of generating tree-structured scenarios, these methods evolve the stochastic process to generate complete 
paths (Dupačová et al., 2000) in a "fan" structure. A procedure called clustering or bucketing must be employed (Kaut and Wallace, 2007) to transform a fan into a scenario tree if the tree structure is preferred in an application. Alternatively, scenarios can be generated by a moment matching method even without knowing the marginal distribution functions exactly (Høyland and Wallace, 2001; Smith, 1993; Kouwenberg, 2001; Høyland et al., 2003). This method contours the marginal distribution functions by their moments (mean, variance, skewness, etc.) or other statistical properties. In particular, Høyland and Wallace (2001) proposed a moment matching method, which we use in this paper, to generate a finite number of scenarios with a tree structure. The underlying idea is to construct scenarios and their corresponding probabilities by approximating the specified statistical properties according to the available degrees of freedom. This technique has also been applied to generate scenarios for spot prices and prediction errors based on the first four central moments (Fleten and Pettersen, 2005). Several scenario reduction methods have been developed in the past decade. The idea in (Dupačová et al., 2003; Heitsch and Römisch, 2003) derives from quantitative stability results in terms of a particular probability metric, resulting in two scenario reduction approaches, termed forward selection and backward reduction. Heitsch and Römisch (2003) extends the original idea addressed in (Dupačová et al., 2003), and improves the iterative procedure, achieving a new version of forward selection and simultaneous backward reduction with considerable performance improvement. These scenario reduction techniques have been used in many power systems applications of stochastic programming, such as for power management (Growe-Kuska et al., 2003; Morales et al., 2009). However, they are based on upper bounds of the particular probability metrics in form of certain mass transportation problems rather than on the particular metrics themselves. Heitsch and Römisch (2007) extends the earlier work by relying directly on the probability metrics. Based on stability behavior of multi-stage stochastic programming models, Heitsch and Römisch (2011) argues that scenario tree reduction in a multi-stage model should not only rely on $L_{r}$-norms, and Heitsch and Römisch (2009b) derives a new scenario reduction method motivated by the idea mentioned in (Heitsch and Römisch, 2011).

The probability-based scenario reduction method focuses on only the scenario parameters, but fails to account for where the uncertainties appear in the mathematical formulation and their impacts on the solution. To incorporate the influences of scenarios on the first-stage decisions in the reduction process, a new heuristic scenario reduction method FSWC (Wang, 2010) is applied in this paper. An imple- 
mentation challenge is to track the influences of the stochastic parameters on every first-stage variable in a large-scale problem. Thus, it is reasonable to concentrate on a subset of first-stage decisions that dominate the others or can best reflect the essences of different scenarios. Accordingly, identification of key first-stage decisions is required. The details of identifying these key first-stage decisions in our GEP application are discussed in Section 2.6.

A number of papers focus on GEP, with scopes that range from problem formulation to solution algorithms. Due to the progress towards deregulation and competition, the conventional GEP models in some studies have been replaced by decentralized ones. Several papers have compared the optimal investment of centralized environment to that of decentralized environment, and developed new models and algorithms by including game theory and uncertain factors (Roh et al., 2007; Botterud et al., 2005; Chuang et al., 2001; Jin and Ryan, 2011). However, even in a decentralized environment, a centralized planning process can provide targets. Once these targets are identified, incentives and regulations can be designed to encourage decentralized decision makers to approach one of them (Jin and Ryan, 2011). Because the restructuring procedure has not taken hold in all regions of the US and large utilities still develop their own expansion plans, we maintain focus on this paper on a cost-minimization formulation that involves several uncertain factors. Among the papers addressing the centralized GEP issues, (Gorenstin et al., 1993) is one of the earliest papers to discuss minimizing the investment and operational cost under uncertainties. However, it only covers the formulation of a stochastic program for minimizing the expected cost, and ignores the computational issues related to scenarios. Park et al. $(1999,2000)$ serve the same purpose, but account for more factors - available capacities of each generation technology, loss of load probability and reserve margin. Nevertheless, they not only ignore the uncertainties of power demands and fuel prices, but also fail to guarantee that their solutions resulting from metaheuristic methods are optimal. To achieve a more reliable expansion strategy minimizing expected total cost for long-term centralized GEP under uncertainties, this paper shares a similar two-stage stochastic GEP model with (Jin et al., 2011). The model minimizes the sum of expected investment, operation cost and penalty for unmet energy while considering the balance of system demand over the whole planning horizon and some limitations on the number of generators added of each type. Rather than sampling scenarios as in (Jin et al., 2011), we explore the performance of the FSWC heuristic in this paper. 
Because a GEP model often includes a number of discrete or nonlinear components, implicit enumeration may be required to obtain the optimal investment strategy. Dynamic programming (DP) has been widely used for solving such GEP models (Dapklus and Bowe, 1984; Mo et al., 1991), but the so-called "curse of dimensionality" often hinders its direct application in GEP especially when uncertainties are involved. As does stochastic programming (SP), DP requires finite sets of discrete states, which correspond to nodes in the scenario tree, and can capture the dynamic aspects of a problem. DP allows general nonlinear costs (Mo et al., 1991), but SP is more flexible with respect to state variables (Kall and Wallace, 1994). Large scale SP formulations of GEP are also amenable to decomposition methods that can exploit the special structure of subproblems (Bloom, 1982; Dantzig et al., 1989).

\subsection{Generation expansion planning model}

The traditional GEP model aims to determine the ideal technologies, expansion sizes, and timing of new generator construction in an economic manner, while ensuring sufficient capacity to satisfy the projected demand growth (Gonen, 1988). As explained above, we investigate generation expansion from the centralized planning point of view in this paper. Our primary focus is to investigate the performance of our new scenario reduction method in this context. Developing a long term investment plan requires a model that includes extensive uncertainties. Among these uncertainties, electricity demand growth is often modeled as a stochastic process. Several additional sources of uncertainty such as the fuel cost, construction time, equipment outage rates, and government policies may also affect future planning activities. We neglect the equipment outages in our GEP model, and assume that government policies are stable. Thus, fuel cost is the other major uncertainty considered in this chapter. According to the information provided by the Energy Information Administration (EIA) of the US Department of Energy, coal and natural gas are the major fuels for electricity generation in the US, with coal accounting for $45 \%$ and gas used to generate $23 \%$ of electricity generated in 2009 (U.S. Energy Information Administration, 2010e). The price of coal has been relatively stable for the past 20 years but natural gas price has fluctuated considerably more (U.S. Energy Information Administration, 2010c,d). In this paper, we consider annual electricity demand and average natural gas price as the only uncertain quantities. Following the above assumptions, a simplified GEP model will be formulated after presenting 
the notations used in the whole chapter.

Sets:

$\mathscr{I} \quad$ Scenarios set

$\mathscr{T}$ Years set

$\mathscr{G}$ Generators set

\section{Indices:}

$i \quad$ Index for scenarios

$t \quad$ Index for years in the planning horizon

$g \quad$ Index for generator types

$\ell$ Index for branches from a node

\section{Decision variables:}

$U_{g t} \quad$ The number of generators of type $g$ to be built in year $t$, integer

$E_{g t i} \quad$ Energy provided by generator type $g$ in year $t$ under scenario $i, M W h$

$U E_{t i} \quad$ Unserved energy in year $t$ under scenario $i, M W h$

A simplified two-stage GEP model is formulated as (2.1)-(2.5):

$$
\begin{array}{ll} 
& \min _{U_{g t}, E_{g t i}, U E_{t i}} \sum_{i} p_{i} \xi_{i} \\
& \xi_{i}=\frac{1}{(1+r)^{t-1}}\left(\sum_{t} \sum_{g}\left(b_{g}+f m_{g}\right) m_{g}^{\max } U_{g t}+\sum_{t}\left(\sum_{g} c_{g t i} E_{g t i}+P c U E_{t i}\right)\right), \forall i \in \mathscr{I} \\
\text { s.t. } \quad & \sum_{g} E_{g t i}+U E_{t i}=d_{t i}, \forall t \in \mathscr{T}, i \in \mathscr{I} \\
& E_{g t i} \leq H_{t}\left(n_{g} m_{g}^{\max } \sum_{k \leq t} U_{g k}+I_{g}\right) \forall g \in \mathscr{G}, \forall t \in \mathscr{T}, \forall i \in \mathscr{I} \\
& \sum_{t} U_{g t} \leq u_{g}^{\max }, \forall g \in \mathscr{G}
\end{array}
$$

Formula (2.1) is the objective function which indicates the purpose of identifying an expansion and generation plan that achieves the minimum expected cost over all possible scenarios. The expected discounted cost in formula (2.2) includes investment cost, generation (including maintenance) cost and penalty cost from unserved energy over the whole planning horizon. The investment decisions, $U_{g t}$ are the first-stage "here and now" decision variables, while $E_{g t i}$ and $U E_{t i}$ are the second-stage "wait and see" decisions that depend on the scenario realization. Equation (2.3) requires that the annual electricity demand is the sum of provided energy and the unserved energy in each scenario. Constraint (2.4) represents capacity constraints of existing and new generators. Due to financial capacity, environmental 


\section{Parameters:}

$b_{g} \quad$ Total cost to build a generator of type $g$, discounted to beginning of construction period, $\$ / M W$

$\mathrm{fm}_{\mathrm{g}} \quad$ Fixed O\&M cost of generator type $g, \$ / M W$

$I_{g} \quad$ The total generation capacity of generator of type $g$ at the beginning of the planning horizon

$u_{g}^{\max } \quad$ The maximum number of generators of type $g$ to be built over the planning horizon

$m_{g}^{\max }$ Installed capacity of a new type $g$ generator, $M W$

$H_{t} \quad$ Total hours in year $t$

$r \quad$ Annual interest rate for cost discounting

$N \quad$ The total number of generated scenarios

$n_{g} \quad$ Capacity factor of type $g$ generator

$p_{i} \quad$ The probability that scenario $i$ occurs

$P c \quad$ Penalty for unserved energy, $\$ / M W h$

$c_{g t i} \quad$ Generation cost of generator type $g$ in year $t$ under scenario $i, \$ / M W h$

$d_{t i} \quad$ Annual electricity demand in year $t$ under scenario $i, M W h$

impacts, and other drivers, we bound the total number of each type of generator added, as formulated in $(2.5)$.

\subsection{Scenario generation}

It is generally unreasonable to solve a mathematical program with uncertain parameters described by continuous distributions. The common approach is to form an approximation of the original continuous stochastic process or underlying distribution by discretization. The procedure of discretizing the continuous distribution or stochastic process is termed scenario generation. We choose a tree or lattice rather than a fan structure in this paper because the size of a tree or lattice can be controlled by its structure.

In general, a scenario tree is a set of nodes and branches used in models of decision making under uncertainty. Every node in the tree represents a possible state of the world at a particular time point and a position where a decision can be made. Each tree node has only a single predecessor, but can have multiple successors, whereas a lattice node can have multiple predecessors. An arc emanating from a node indicates a possible realization of the uncertain variables from that state. The first node in the scenario tree is defined as the root node, representing the initial situation, being in this paper the values of annual electricity demand and natural gas price in the current year. Any possible forward path from the root node to a node at the last time point is defined as a scenario which describes a plausible realization of annual electricity demands and natural gas prices over the time horizon. The number of 
nodes in a tree at the final time point equals the total number of scenario paths. The nodes shared in a lattice illustrate that a plausible state may be achieved from the initial condition by multiple realization paths. Although the number of paths in the lattice is similar to the number of branches in the tree, the nodes at the final time point will be much fewer than the scenarios.

\subsubsection{Optimization model for scenario tree generation}

The moment matching method of generating scenarios (Høyland and Wallace, 2001) was adopted to generate the immediate successors from each node. The procedure to generate the whole scenario tree can be recursively applied from the root node to the nodes at the end of the final period. Since the proper statistical properties can partially describe the underlying distribution of data, we minimized a measure of distance between statistical properties of the fitted discrete distribution and the statistical specifications, subject to a constraint restricting the conditional branching probabilities to sum to one. Examples of statistical specifications are mean, variance, and skewness. We assume the statistical specifications of random variables have been obtained. The details of their computation for the case study are shown in Section 2.6. Let $S$ be the set of all specified statistical specifications, and $S_{V A L_{j}}$ be the value of the $j$ th statistical specification. The random variables corresponding to the same period are grouped into a vector, called the scenario node vector, denoted by $z_{\ell}$, where $\ell$ indicates the $\ell$ th branch. The corresponding conditional probability of the $\ell$ th branch is denoted by $\pi_{\ell}$. Notation $z_{0}$ represents annual electricity demand and natural gas price at the root node, and $\pi_{0}$ is 1 . The $j$ th statistical property of the fitted approximating distribution is denoted as $f_{j}(z, \pi)$. For example, if $f_{j}(z, \pi)$ represents mean, then it is computed as $\sum_{\ell} z_{\ell} \pi_{\ell}$. Thus, the general scenario generation model based on optimization may be formulated as follows (Høyland and Wallace, 2001), where the common square norm is adopted to measure the distance.

$$
\begin{array}{cc} 
& \min _{z \ell}, \pi_{\ell} \sum_{j}\left(w_{j}\left(f_{j}(z, \pi)-S_{V A L_{j}}\right)^{2}\right. \\
\text { s.t. } & \sum_{\ell} \pi_{\ell}=1 \\
& \pi_{\ell} \geq 0
\end{array}
$$

A set of weights $w_{j}$ represent the importance of satisfying different statistical specifications, which 
can be manually specified by individual preference. In general, model (2.6)-(2.8) must be solved at every node in the scenario tree except those at the last time point. However, if the stochastic processes describing the uncertain data are stationary, the solution obtained at the root node can be applied at every node. The nonconvex nonlinear program (2.6)-(2.8) was first solved at the root node by the Matlab fmincon function. Because we often found solutions with nonzero objective values (indicating that the statistical properties of the fitted distribution are not close to the statistical specifications), we tried multiple starting points, and selected the solution with the smallest objective value (near zero).

\subsubsection{Controlling the size of scenario tree}

Høyland and Wallace (2001) discussed potential pitfalls, such as underspecification and overspecification, when using the moment matching method to generate scenarios, and proposed an idea based on counting the degrees of freedom to control the size of scenario tree while avoiding the pitfalls. The idea is expressed in formula (2.9), where the symbol " " denotes "close to":

$$
(D+1) y-1 \sim \text { the number of specifications }
$$

where $D$ indicates the dimension of each scenario node vector, so that $D+1$ represents the number of random variables plus the branch probability; and $y$ represents the number of branches from each node. Since constraint (2.7) eliminates one degree of freedom, $(D+1) y$ is reduced by one to get the final degrees of freedom. A simple example of calculating the number of branches is illustrated. Suppose a period includes one year, and the two random variables, the annual electricity demand and natural gas price, are specified by their means, variances, skewnesses and correlation. Then the number of specifications is 7 . According to (2.9), we have $(2+1) y-1 \sim 7$, so $y$ should be 3 . If the branches cover a two-year period, the number of specifications is 14 , since four random variables are involved - two for annual electricity demands and two for average annual natural gas prices. The number of branches from a node should still be 3 , as $(4+1) \times 3-1=14$. Because the long time horizon with

annual periods results in a large scenario tree, we use longer periods in the later years of the case study to decrease the number of generated scenarios, as described in detail later. 


\subsection{Scenario reduction}

The computational effort for solving scenario-based stochastic program depends mainly on the number of scenarios even if decomposition techniques are used. The huge number of scenarios that frequently result from the scenario generation process limits tractability. Thus, it is essential to approximate the original scenarios with a much smaller subset that can well approximate the original scenario set. Existing scenario reduction methods control the approximation's goodness-of-fit according to probability metrics. The common scenario reduction methods, forward selection (FS), backward reduction and their variants, obtain the selected scenarios by recursive pairwise comparisons of the distances between scenarios. We review the fast forward selection (FFS) method as follows (Heitsch and Römisch, 2003). A scenario here is defined as a path from the root node to a node in the last stage, denoted as $\omega_{i}, i=1, \cdots, N$, and its corresponding probability by $p_{i}$ which is the product of conditional probabilities over the path. In the following, $J^{[s]}$ represents the scenario subset from which the next scenario will be selected after the sth selection and $\eta(\cdot)$ is a nonnegative, continuous and symmetric function, usually defined as some norm on $\mathbb{R}^{n}$. We use the $L_{2}$-norm. The intuitive idea of the FFS method is to select a subset $\Omega^{\prime}$ from the original finite scenario set $\Omega$ so that $\Omega^{\prime}$ is the subset of the prescribed size that has the shortest distance to the remaining scenarios. The FFS method, which focuses on the selection of $n$ scenarios beginning from an empty set, includes the following steps:

\section{Fast forward selection (FFS):}

1. Let $s=1$, calculate the distances of all scenario pairs $\delta_{i, k}^{[1]}=\eta\left(\omega_{i}, \omega_{k}\right), i, k=1, \cdots, N$, and compute the weighted distance of each scenario to the other scenarios $z_{k}^{[1]}=\sum_{i \neq k} p_{i} \delta_{i, k}^{[1]}, k=1, \cdots, N$. Select $k_{1}=\arg \min _{k \in 1, \cdots, N} z_{k}^{[1]}$, and set $J^{[1]}=\{1, \cdots, N\} \backslash\left\{k_{1}\right\}$;

2. Let $s=s+1$, and replace the scenario pair distance with the smaller one of the original pair distance and the distance to the selected scenario in $(s-1)$ th selection, $\delta_{i, k}^{[s]}=\min \left\{\delta_{i, k}^{[s-1]}, \delta_{i, k_{s-1}}^{[s-1]}\right\}$, $i, k \in J^{[s-1]}$. Compute $z_{k}^{[s]}=\sum_{i \in J^{[s-1]} \backslash\{k\}} p_{i} \delta_{i, k}^{[s]}, k \in J^{[s-1]}$ for every candidate scenario in the $s$ th selection, and choose $k_{s}=\arg \min _{k \in J^{[s-1]}} z_{k}^{[s]}, J^{[s]}=J^{[s-1]} \backslash\left\{k_{s}\right\}$, the one that minimizes the weighted distance to the remaining scenarios;

3. If the number of the selected scenarios is less than $n$, return to Step 2; 
4. Add to the probability of each selected scenario the sum of the probabilities of all unselected scenarios that are close to it; i.e. $q_{j}=p_{j}+\sum_{i \in L(j)} p_{i}$, for any $j \in \Omega^{\prime}$, where $\Omega^{\prime}$ is the set of selected scenarios, and $L(j):=\left\{i \in \Omega \backslash \Omega^{\prime}, j=j(i)\right\}, j(i)=\arg \min _{j \in \Omega^{\prime}} \eta\left(\omega_{i}, \omega_{j}\right)$ for any $i \in \Omega \backslash \Omega^{\prime}$.

Note that FFS only accounts for the paths $\left\{\omega_{i}\right\}$ and their corresponding probabilities $\left\{p_{i}\right\}$, but not for the decision variables, cost function or where the uncertainties appear in the mathematical programming formulation. Thus, FFS is a problem independent scenario reduction method, which will lead to the same subset of scenarios no matter whether the uncertain parameters exist in cost coefficients, constraint right-hand sides or the constraint coefficient matrix. Moreover, dramatic differences among the magnitudes of the uncertain parameters may distort the distance metric. Therefore, we use an alternative new heuristic scenario reduction method that considers the impacts of scenarios on first-stage decision variables instead of directly selecting scenarios based only on distances among them. The heuristic scenario reduction method we use here is called forward selection in wait-and-see clusters (FSWC) (Wang, 2010). As mentioned before, FSWC aims to incorporate the impact of each scenario on first-stage decision variables and, thus, may be especially effective when problems are solved repeatedly over with a rolling horizon. To measure the impacts of scenarios on the first-stage decision variables, a deterministic wait-and-see problem is defined for an individual scenario, $k$, by setting scenario probability $p_{k}=1$, and $p_{i}=0, i \neq k$, in (1)-(5), and deleting constraints (2)-(4) for $i \neq k$. The solution includes scenario-specific optimal values of the first-stage decisions.

This deterministic wait-and-see problem is solved for each scenario and the corresponding firststage decisions are recorded. Because a large scale stochastic program often includes a large number of first-stage variables, it is difficult to track the impact on each of them. Therefore, it is reasonable to focus on the impact on the so-called key first-stage variables which were originally defined in (Wang, 2010) to be those variables that determine the other first-stage variables. As a simple example, if there are only two kinds of variables involved in first-stage variables, where one is the fuel purchased from the market in each period and the other is the power generated from a given power plant, then fuel amounts purchased will be key variables since the amount of fuel limits the amount of generation. The definition of key first-stage variables is extended here to include those variables that differ significantly according to scenarios. For example, if the planned numbers of power plants to exist in each year are 
the only first-stage variables in a stochastic program, and the electricity demand in every year is the only uncertain parameter, then the planned number of power plants in the last few years of the planning horizon can be considered as key first-stage variables, as they represent the targets of the expansion plan. The ending numbers of power plants can reflect the trend of the electricity demand more clearly than the planned numbers of plants in earlier years. The FSWC method implements forward selection within clusters of scenarios that are formed according to the similarity of those key first-stage variables. The clustering process is repeated as necessary to force the number of clusters to satisfy the desired cardinality of the reduced scenario set. Suppose the two-stage stochastic program can be formulated as (2.10) - (2.14):

$$
\begin{gathered}
\min _{x} c^{\top} x+\mathscr{Q}(x, \mathscr{I}) \\
\text { s.t. } \quad A x=b \\
x \text { binary }
\end{gathered}
$$

where

$$
\begin{aligned}
\mathscr{Q}(x, \mathscr{I}) & =E_{\mathscr{I}}[Q(x, i)] \\
Q(x, i) & =\min _{y_{i}}\left\{q_{i}^{\top} y_{i} \mid W y_{i}=h_{i}-T_{i} x\right\} .
\end{aligned}
$$

As in the FFS method, the prescribed cardinality is set to $n$, then the details of the FSWC procedure are presented below:

\section{Forward selection in wait-and-see clusters (FSWC):}

1. Solve problem (2.15) - (2.18) for each scenario $i$, and retain the key first-stage decisions $\hat{x}_{i}$

$$
\begin{gathered}
\min _{x_{i}, y_{i}} c^{\top} x_{i}+q_{i}^{\top} y_{i} \\
\text { s.t. } A x_{i}=b \\
T_{i} x_{i}+W y_{i}=h_{i} \\
x_{i} \text { binary }
\end{gathered}
$$

2. Group scenarios into the same cluster, $C_{l}=\left\{i \mid \hat{x}_{j}=\hat{x}_{k}, \forall i, k \in I_{l}\right\}, l=1,2, \cdots, n_{m}$; if $n_{m}<=n$, go to step 4; 
3. Cluster the $n_{m}$ groups of $\left\{C_{l}\right\}_{n_{m}}$ into $n$ clusters by applying $k$-means method on $\hat{x}_{l}$ with an appropriate norm (e.g. $L_{2}$ norm) (Mathworks, 2011), and create the corresponding $n$ clusters of original scenarios at the same time;

4. Use the FFS method to select one scenario from each cluster of original scenarios.

\subsection{Case study}

We implemented the GEP model (2.1)-(2.5) on a hypothetical system involving six types of generators over a 20 year horizon. The data for annual electricity demands and natural gas prices from 1973 to 2010 were collected from The Energy Information Administration (U.S. Energy Information Administration, 2011, 2010b). The related data of each type of generators in the system were collected from reports (U.S. Energy Information Administration, 2008, 2010a; Tidball et al., 2010), and are given in Table 2.1. The generator types include baseload (coal), gas combined cycle (CC), gas combustion turbine (CT), nuclear, wind (farm) and integrated gasification combined cycle (IGCC). The building costs were obtained by following the computation method illustrated in (Jin et al., 2011). Because there is no evident intention to expand hydropower in the U.S. Midwest in the next decades (JCSP, 2012; U.S. Energy Information Administration, 2012a), we did not include it as an expansion option in our case

study. The maximum numbers of planned units of each type, $u_{g}^{\max }$, were set to reflect some practical considerations such as difficulty with obtaining permits for nuclear plants and hesitation to include too much wind generation because of its variable and intermittent nature. Restrictions on the other types of generators are relaxed in (Feng and Ryan, 2012), where additional characteristics of wind units, temporal variability in load, and risk considerations are also included.

As mentioned in Section 2.3, only annual electricity demand and average natural gas price were considered as random variables. To generate plausible scenarios, it is necessary to assume appropriate underlying distributions or stochastic processes for these two random variables. Similar to (Jin et al., 2011), but using updated data sets, correlated GBM processes were selected based on historical goodness of fit. The GBM process has often been invoked as a model for disparate qualities in financial markets, such as stock prices, natural resource prices, and the increasing rate of demand for products (Marathe and Ryan, 2005). The following subsections describe verification of the assumption that GBM 
Table 2.1 The parameters for candidate generators \& existing generation capacity

\begin{tabular}{r|r|r|r|r|r|r}
\hline \hline Generator type $g$ & Base-load(coal) & CC & CT & Nuclear & Wind & IGCC \\
\hline$m_{g}^{\max }$ & 600 & 400 & 230 & 1350 & 50 & 380 \\
$n_{g}(\%)$ & 84.6 & 84.6 & 80.0 & 90.2 & 30.0 & 81.0 \\
$c_{g 0}(\$ / M W h)$ & 14.5 & 22.6 & 37.8 & 0.4 & 0 & 11.1 \\
Growth rate of $c_{g 0}(\%)$ & 3 & $*$ & $*$ & 3 & 0 & 3 \\
$u_{g}^{\max }$ & 45 & 60 & 90 & 8 & 105 & 45 \\
$I_{g}(M W)$ & 21375 & 11000 & 11000 & 8800 & 2450 & 11250 \\
$f_{m g}(\$ / M W)$ & 28150 & 11960 & 10770 & 92040 & 30980 & 47150 \\
$b_{g}(\$ / M W)$ & 964922 & 371102 & 312963 & 1336759 & 895283 & 1078495 \\
\hline
\end{tabular}

*: generation cost of $\mathrm{CC}$ and $\mathrm{CT}$ are scenario dependent parameters.

Table 2.2 Statistical properties of log-ratios of annual electricity demand and natural gas price

\begin{tabular}{r|r|r|r|r}
\hline \hline & Mean & Std Dev & Shapiro-Wilk & p-value \\
\hline Electricity demand & 0.0214 & 0.0227 & 0.9571 & 0.1753 \\
Natural gas price & 0.0782 & 0.2503 & 0.9473 & 0.0782 \\
\hline
\end{tabular}

fits both the annual electricity demands and natural gas prices, scenario generation for these two random variables, and a comparison between the FFS and FSWC scenario reduction methods.

\subsubsection{Geometric Brownian motion assumption and verification}

According to (Ross, 2010), if $Y(t)$ is a Brownian motion with drift coefficient $\mu$ and variance parameter $\sigma^{2}$, then the stochastic process $X(t)=e^{Y(t)}$ follows a GBM. Because the GBM has a property that its $\log$ ratio, $\log \left(\frac{X(t+1)}{X(t)}\right)$, follows a normal distribution, it is necessary to verify normality of the annual growth log ratios of each variable's time series. Second, we can verify the independent increments of the underlying Brownian motion process by examining the autocorrelations of the log ratios. The JMP statistical software package was employed for these tests. The relevant parameters are presented in Table 2.2.

The last two columns in Table 2.2 present the Shapiro-Wilk test statistics computed by JMP. The $\mathrm{p}$-values for both series indicate failure to reject the null hypothesis that each data set is sampled from a normal distribution. Autocorrelation functions were computed to check the independence among successive log-ratios of each variable as well. As Fig.2.1 illustrates, the insignificant spikes at all lags for the sample auto-correlation function (ACF) indicate that there is no significant autocorrelation 
among log-ratios of either time series. Hence, it is reasonable to assume both electricity demands and natural gas prices satisfy GBM process. In addition, we used JMP to compute a correlation between electricity demand and natural gas price over the past years as 0.7774 . According to these models and parameters, scenarios of electricity demands and natural gas prices could be generated based on the method described in Section 2.4. The following subsection illustrates the steps of specifying statistical properties and then generating scenarios accordingly.
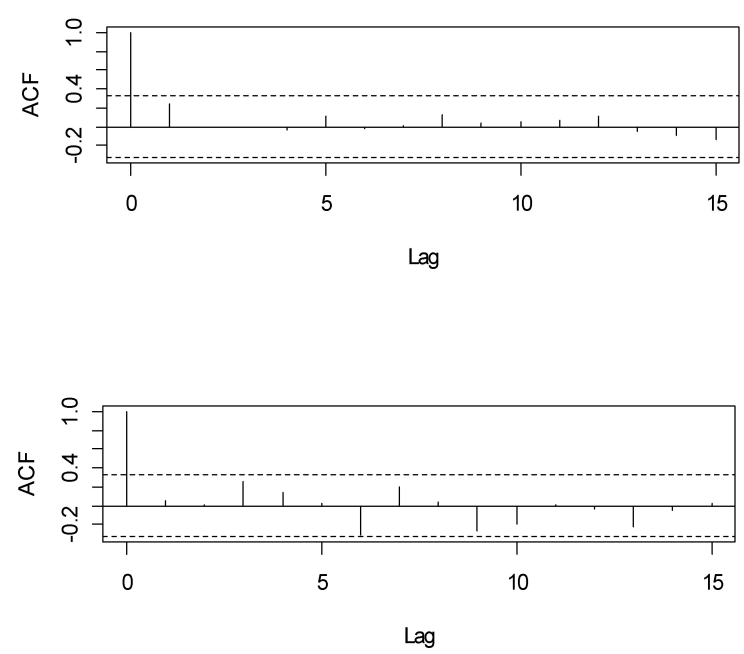

Figure 2.1 Sample ACF of log growth ratios: (up) annual electricity demand, (down) natural gas price

\subsubsection{Important properties of GBM for scenario generation}

The first step in generating scenarios by the moment matching method is to specify statistics, such as moments and correlations. According to formulas (2.6)-(2.8), for each scenario tree node, the values of important statistical specifications of its successors must be pre-calculated given the information about that node, including the lengths of the time periods between successive nodes. We included mean, variance, and skewness of each random variable as well as the correlation between them in the set of statistical specifications. According to the GBM process properties, the conditional central moments can be obtained explicitly. The moment generating function of a Brownian motion with drift is (Sigman, 2006): 


$$
M_{Y(t)}(s)=E\left[e^{s Y(t)}\right]=e^{\mu t s+\sigma^{2} s^{2} t / 2}, \forall-\infty<s<\infty
$$

It follows that the first two moments are given by:

$$
\begin{aligned}
& E[X(t)]=X_{0} e^{\left(\mu+\sigma^{2} / 2\right) t} \\
& E\left[X^{2}(t)\right]=X_{0}^{2} e^{\left(\mu+2 \sigma^{2} t\right)}
\end{aligned}
$$

Thus, the variance and skewness of a GBM process are:

$$
\begin{gathered}
\operatorname{Var}[X(t)]=X_{0}^{2} e^{2 \mu t+\sigma^{2} t}\left(e^{\sigma^{2} t}-1\right) \\
\operatorname{skew}[X(t)]=\left(e^{\sigma^{2} t}+2\right) \sqrt{e^{\sigma^{2} t}-1}
\end{gathered}
$$

Given the history of a GBM process up to time $s$, for $s<t$, the conditional expectation of $X(t)$ can be found as:

$$
\begin{aligned}
E[X(t) \mid X(u), 0 \leq u \leq s] & =E\left[e^{Y(t)} \mid Y(u), 0 \leq u \leq s\right] \\
& =E\left[e^{Y(s)+Y(t)-Y(s)} \mid Y(u), 0 \leq u \leq s\right] \\
& =e^{Y(s)} E\left[e^{Y(t)-Y(s)} \mid Y(u), 0 \leq u \leq s\right] \\
& =X(s) E\left[e^{Y(t)-Y(s)}\right] .
\end{aligned}
$$

From the normal distribution of $Y(t)-Y(s) \sim N\left(\mu(t-s), \sigma^{2}(t-s)\right)$, equation (2.24) can be rewritten as:

$$
X(s) E\left[e^{Y(t)-Y(s)}\right]=X(s) e^{\left(\mu+\sigma^{2} / 2\right)(t-s)}
$$

which expresses the stationary increments of the underlying Brownian motion process. Similarly, the conditional variance and conditional skewness of $X(t)$ can also be derived, given a previous value $X(s)$ :

$$
\begin{aligned}
\operatorname{Var}[X(t) \mid X(u), 0 \leq u \leq s] & =\operatorname{Var}\left[e^{Y(t)} \mid Y(u), 0 \leq u \leq s\right] \\
& =\operatorname{Var}\left[e^{Y(s)+Y(t)-Y(s)} \mid Y(u), 0 \leq u \leq s\right] \\
& =e^{2 Y(s)} \operatorname{Var}\left[e^{Y(t)-Y(s)} \mid Y(u), 0 \leq u \leq s\right] \\
& =X(s)^{2} \operatorname{Var}\left[e^{Y(t)-Y(s)}\right] .
\end{aligned}
$$


Thus,

$$
\operatorname{Var}[X(t) \mid X(u), 0 \leq u \leq s]=X(s)^{2} e^{2 \mu(t-s)+\sigma^{2}(t-s)}\left(e^{\sigma^{2}(t-s)}-1\right),
$$

and for conditional skewness, we have

$$
\begin{aligned}
\operatorname{skew}[X(t) \mid X(u), 0 \leq u \leq s] & =\operatorname{skew}\left[e^{Y(t)} \mid Y(u), 0 \leq u \leq s\right] \\
& =\operatorname{skew}\left[e^{Y(s)+Y(t)-Y(s)} \mid Y(u), 0 \leq u \leq s\right] \\
& =E\left[e^{Y(s)}\left(e^{Y(t)-Y(s)}-E\left[e^{Y(t)-Y(s)}\right]\right)\right]^{3} /\left(\operatorname{Var}\left(e^{Y(s)} e^{Y(t)-Y(s)}\right)\right)^{\frac{3}{2}} \\
& =\left(e^{\sigma^{2}(t-s)}+2\right) \sqrt{e^{\sigma^{2}(t-s)}-1} .
\end{aligned}
$$

According to (2.19)-(2.28), the conditional central moments can be computed for any length interval between the two time points.

The stationarity of the GBM process simplifies the scenario generation procedure. Rather than solving (2.6)-(2.8) at each node, the nonlinear optimization problem can be solved once for a given interval length and the results applied to each node whose branches span an interval of that length.

\subsubsection{Division of the planning horizon and scenario tree generation}

Even though the number of branches from each scenario node is controlled, the number of subperiods in the planning horizon also influences the size of scenario tree. In our 20-year case study, if every year in the planning horizon is viewed as an individual period, and just two realizations branch from each node, there would be $2^{20}$ leaves of the scenario tree. Thus, we wish to divide the planning horizon into fewer periods. In this paper, 20 years were divided into 10 periods with different structure by two methods. As the decision maker may mainly focus on the decisions in the immediate future, the first method is to divide the horizon into increasing length periods - short periods early in the planning horizon and long periods further in the future. Fig.2.2 shows one way of dividing the planning horizon. The second method is to divide the planning horizon into equal length periods. Each of the equal length periods involves two years, as Fig.2.3 presents.

In the first method of dividing the time horizon, each year was considered as a period in the first four years; then two years were viewed as a period in the latter six years; the length of the first three periods in the last ten years is 3 years, and the final period included four years. The different planning 


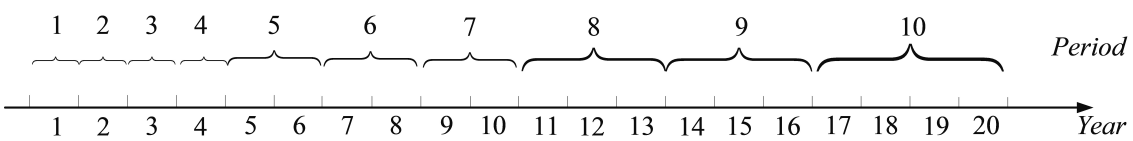

Figure 2.2 Division of the planning horizon time into increasing length periods

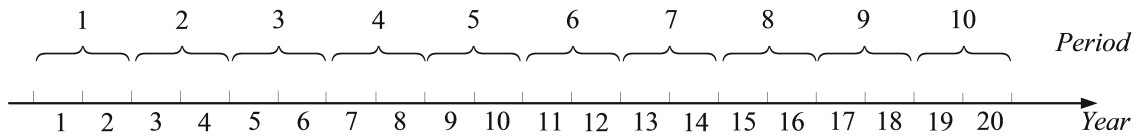

Figure 2.3 Division of the planning horizon time into equal length periods

horizon division methods resulted in different structures of the scenario sets. In the first, according to the notation defined in Section 2.4.2, the nodal dimension $D$ increases from 2 to 8 along the time horizon. In contrast, $D$ is constant in the second method. The number of statistical specifications used in the moment-matching procedure varies with $D$. These specifications are conditional on the realizations in the final year of the predecessor period which is defined as the reference year, and calculated according to (2.24), (2.26) and (2.28). For example, given the annual electricity demand and natural gas price in the reference year, then the specifications $S_{V A L_{j}}$ of those variables in target year $t$ ranging from year $s+1$ to $s+l$ (where $l$ is the length of the subperiod) can be found by using $t-s$ in equations (2.24), (2.26) and (2.28). After identifying the specifications $S_{V A L_{j}}$ and then computing $y$ according to equation (2.9), the nonlinear optimization problem (2.6)-(2.8) is solved at the root node for each subperiod length. Because of the stationarity of the GBM process, the tree can then be built by applying the growth ratios corresponding to each branch from the root node to the respective branches from subsequent nodes according to the subperiod lengths.

The growth rates of annual electricity demand and annual average natural gas price are given in Tables 2.7 through 2.6. Fig.2.4 shows the projections of the tree onto the demand-time plane and the price-time plane.

When generating the second set of scenarios, with equal length subperiods, we first realized possible states for even years, and applied equations (2.24), (2.26) and (2.28) with $t-s$ equal to 2 to compute data values for the subsequent even years. We then applied linear interpolation to find values of annual electricity demands and natural gas prices in odd years. According to equation (2.9), 3 branches were 
Table 2.3 The growth rates of annual electricity demand and natural gas price for periods 1-4

\begin{tabular}{l|r|r|r}
\hline \hline & Demand & Price & Conditional probability \\
\hline Branch 1 & 0.9991 & 0.8759 & 0.5045 \\
Branch 2 & 1.0488 & 1.1807 & 0.2871 \\
Branch 3 & 1.0400 & 1.6067 & 0.2084 \\
\hline
\end{tabular}

Table 2.4 The growth rates of annual electricity demand and natural gas price for periods 5-7

\begin{tabular}{l|r|r|r|r|r}
\hline \hline & \multicolumn{2}{|c|}{$t=s+1$} & \multicolumn{2}{c|}{$t=s+2$} & \multirow{2}{*}{ Conditional probability } \\
\cline { 2 - 5 } & Demand & Price & Demand & Price & \\
\hline Branch 1 & 0.9992 & 0.8812 & 1.0117 & 0.9418 & 0.5084 \\
Branch 2 & 1.0489 & 1.1422 & 1.0712 & 1.0884 & 0.2494 \\
Branch 3 & 1.0417 & 1.5806 & 1.0850 & 2.0423 & 0.0242 \\
\hline
\end{tabular}

made from each node. However, we found two of the three annual growth ratios for annual electricity demand were very similar in the solution to (2.6)-(2.8). Substituting one of the two growth ratios with the other one for demand resulted in little change to value of the objective function. Note that creating two branches for demand and three for gas price allows (2.9) to be satisfied exactly as

$$
2 \text { (demands) }+3 \text { (prices) }+3 \text { (probabilities) }-1=7 \text { (statistical specifications) } .
$$

Moreover, the stationarity and equal length subperiods allowed multiple tree nodes at the same time point to be collapsed into a single lattice node. Table 2.7 shows the growth rates for each branch from a lattice node. Fig. 2.5 shows the demand and price projections of the lattice.

As each set of scenarios covers 10 time periods with three branches from each node, each contains $3^{10}$ scenario paths. Section 2.6.5.1 numerically compares the effects of the two methods on thinning the scenario sets to a manageable size.

\subsubsection{Scenario reduction}

As a baseline for comparison, we applied fast forward selection (FFS) to select scenarios from each original scenario set. To implement the FSWC method, model (2.1)-(2.5) was solved for each scenario individually as a deterministic "wait-and-see" optimization problem by considering the set of scenarios as a singleton and setting that scenario probability to one. The optimal first-stage decisions $\left\{U_{g t}\right\}$ corresponding to each scenario were accumulated over 20 years to get the number of each generator 

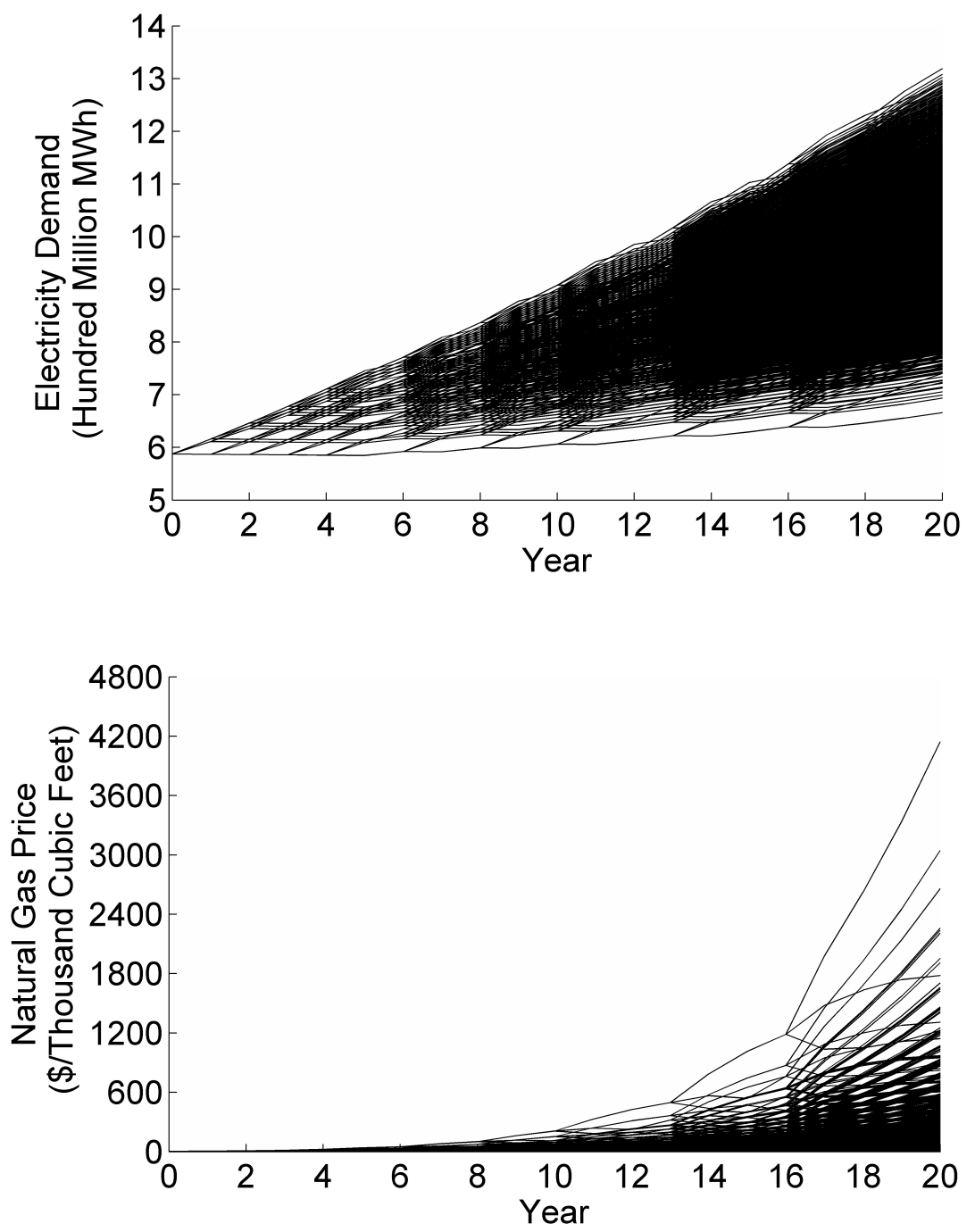

Figure 2.4 Scenario tree with unequal length periods: (up) demand vs. time, (down) gas price vs. time 

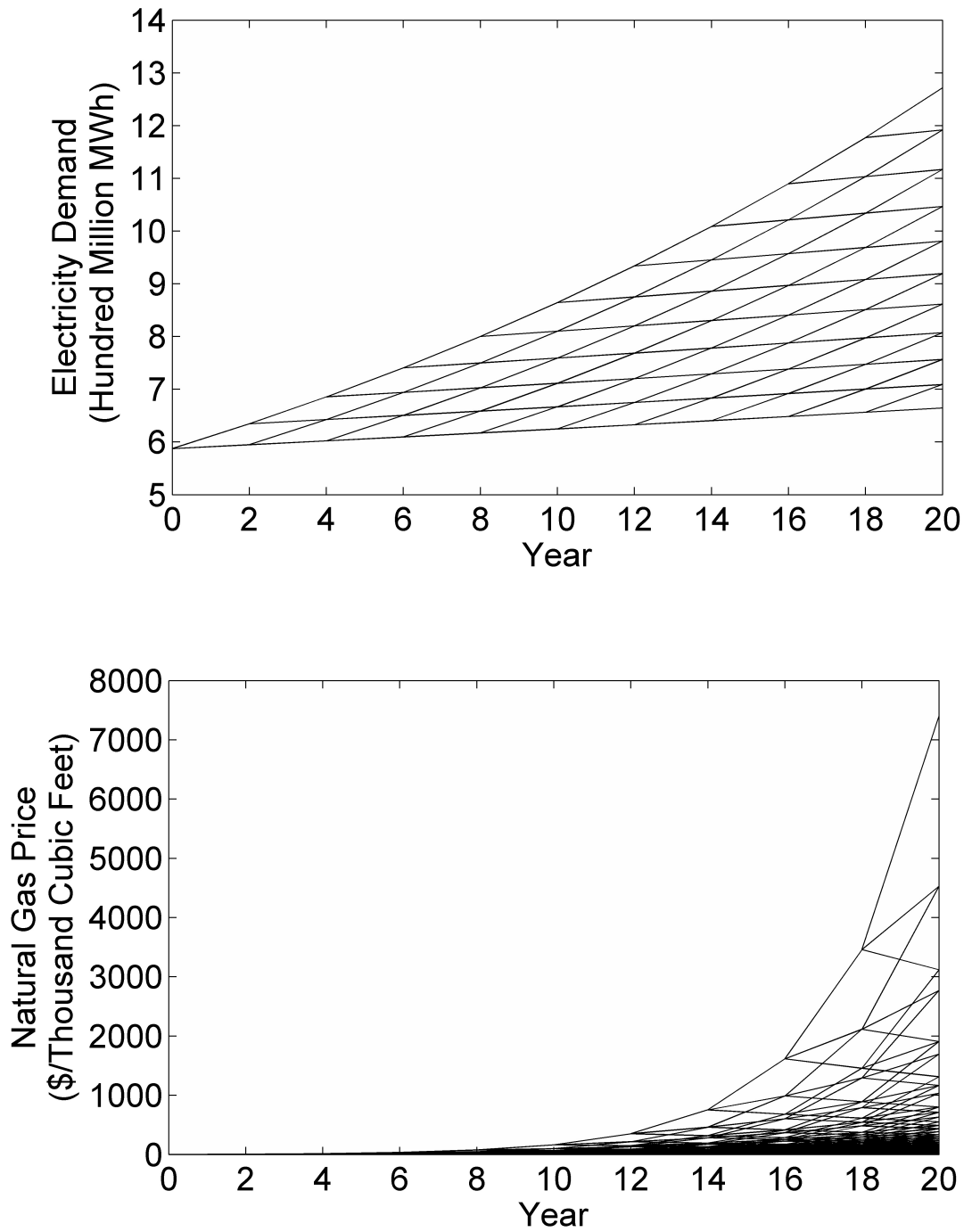

Figure 2.5 Scenario lattice with equal length periods: (up) demand vs. time, (down) gas price vs. time 
Table 2.5 The growth rates of annual electricity demand and natural gas price for periods 8-9

\begin{tabular}{l|r|r|r|r|r|r|r}
\hline \hline & \multicolumn{2}{|c|}{$t=s+1$} & \multicolumn{2}{c|}{$t=s+2$} & \multicolumn{2}{c|}{$t=s+3$} & \multirow{2}{*}{ Cond. Prob. } \\
\cline { 2 - 7 } & Demand & Price & Demand & Price & Demand & Price & \\
\hline Branch 1 & 0.9992 & 0.8812 & 1.0117 & 0.9428 & 1.0265 & 0.8414 & 0.5072 \\
Branch 2 & 1.0489 & 1.1422 & 1.0708 & 1.0839 & 1.1196 & 1.5309 & 0.2499 \\
Branch 3 & 1.0416 & 1.5801 & 1.0852 & 2.0414 & 1.0984 & 2.3866 & 0.2429 \\
\hline
\end{tabular}

Table 2.6 The growth rates of annual electricity demand and natural gas price for period 10

\begin{tabular}{l|r|r|r|r|r|r|r|r|r}
\hline \hline & \multicolumn{2}{|c|}{$t=s+1$} & \multicolumn{2}{c|}{$t=s+2$} & \multicolumn{2}{c|}{$t=s+3$} & \multicolumn{2}{c|}{$t=s+4$} & \multirow{2}{*}{ Cond. Prob. } \\
\cline { 2 - 8 } & Demand & Price & Demand & Price & Demand & Price & Demand & Price & \\
\hline Br. 1 & 0.9995 & 0.8729 & 1.0118 & 0.8862 & 1.0265 & 0.9381 & 1.0430 & 1.0322 & 0.4991 \\
Br. 2 & 1.0483 & 1.2465 & 1.0811 & 1.3756 & 1.1057 & 1.4653 & 1.1329 & 1.5016 & 0.3424 \\
Br. 3 & 1.0392 & 1.6696 & 1.0727 & 2.2109 & 1.1205 & 2.8114 & 1.1590 & 3.4928 & 0.1585 \\
\hline
\end{tabular}

type planned to exist in each year $\left\{V_{g t}\right\}$, where $V_{g t}=\sum_{s=1}^{t} U_{g s}$. Then, the key first-stage variables were defined to be the number of each type of generators in each of the last ten years, and they were formed to be a row vector, i.e. $\left[V_{1,11}, V_{2,11}, \cdots, V_{1,20}, V_{2,20}, V_{3,20}, V_{4,20}, V_{5,20}, V_{6,20}\right]$. The FSWC method described in Section 2.4 can be applied directly on the obtained vectors $\left[V_{i j}\right]$, and the $L_{2}$-norm is adopted in the $k$-means method in Step 3 of FSWC. As mentioned in Section 2.4, these quantities could partially reflect the growth trend of annual electricity demand, as higher annual electricity demand would require more generation capacity. Because the dramatic differences in annual electricity demand appear evident especially in the last ten years according to Fig.4 and Fig.5, we pay more attention to the numbers of generators added in these years. Since more generators of a given type of generator tend to be built if it has lower building cost and lower fuel price, the key first-stage variables could also partially reflect the relative differences between natural gas price and other fuel prices, which were considered as deterministic over the planning horizon. To compare the outputs of FFS and FSWC, various cardinalities

Table 2.7 The growth rates of annual electricity demand and natural gas price for even years in the second scenario tree

\begin{tabular}{l|r|r|r}
\hline \hline & Demand & Price & Conditional probability \\
\hline Branch 1 & 1.0124 & 0.9015 & 0.5237 \\
Branch 2 & 1.0803 & 1.3093 & 0.2969 \\
Branch 3 & 1.0803 & 2.1403 & 0.1794 \\
\hline
\end{tabular}


of selected scenario sets were obtained. Considering the computational complexity to select from the large original scenario sets, the selected sets had modest cardinalities of 10, 20,30, 50 and 100. Because one of the purposes of generation expansion planning is to satisfy demand over the long term, we set the penalty for unserved energy to a large value $P c=10^{7} \$ / M W h$. The scenario reduction procedures and stochastic programming solution process were implemented by using Matlab and CPLEX with the interface provided by Tomlab on computers with $3 \mathrm{GHz}$ CPU.

\subsubsection{Numerical results}

Scenarios selected by FFS and FSWC methods based on the generated scenario sets are presented in this subsection. Comparisons between these two methods were performed by solving (2.1)-(2.5) over a 20 year planning horizon.

\subsubsection{Numerical results for the unequal-length period scenario tree}

Fig.2.6 and 2.7 illustrate the selected scenarios according to FFS and FSWC methods which are projected on the annual electricity demand vs. time and natural gas price vs. time planes, respectively. Because of the space limitation, only the selected sets with cardinality 100 are shown.

According to Fig.2.6 and Fig.2.7, the reduced scenario sets obtained by both reduction heuristics have similar contours with respect to annual electricity demands, but differ somewhat according to the natural gas prices. To make closer comparisons between the reduction methods, expected annual electricity demands and average natural gas prices over the planning horizon based on the two selected subsets as well as the original scenarios have been plotted in Fig.2.8. The expected demands based on each selected set are very similar to the original (Fig.2.8(up)). Fig.2.8(down) illustrates that the expected gas prices according to scenarios selected by the FFS method are closer to the original scenarios than those selected by FSWC. One explanation is that, because of the high penalty for unserved energy, the electricity demand receives more attention in the FSWC method than the natural gas price does. In contrast, the FFS method measures scenario distances by a metric that weights annual electricity demand and natural gas price equally.

To assess the performance of solutions based on the reduced scenario sets, we solved the GEP model (1)-(5) using a reduced scenario set to obtain the optimal first-stage decisions. Tables 2.8 and 2.9, which 

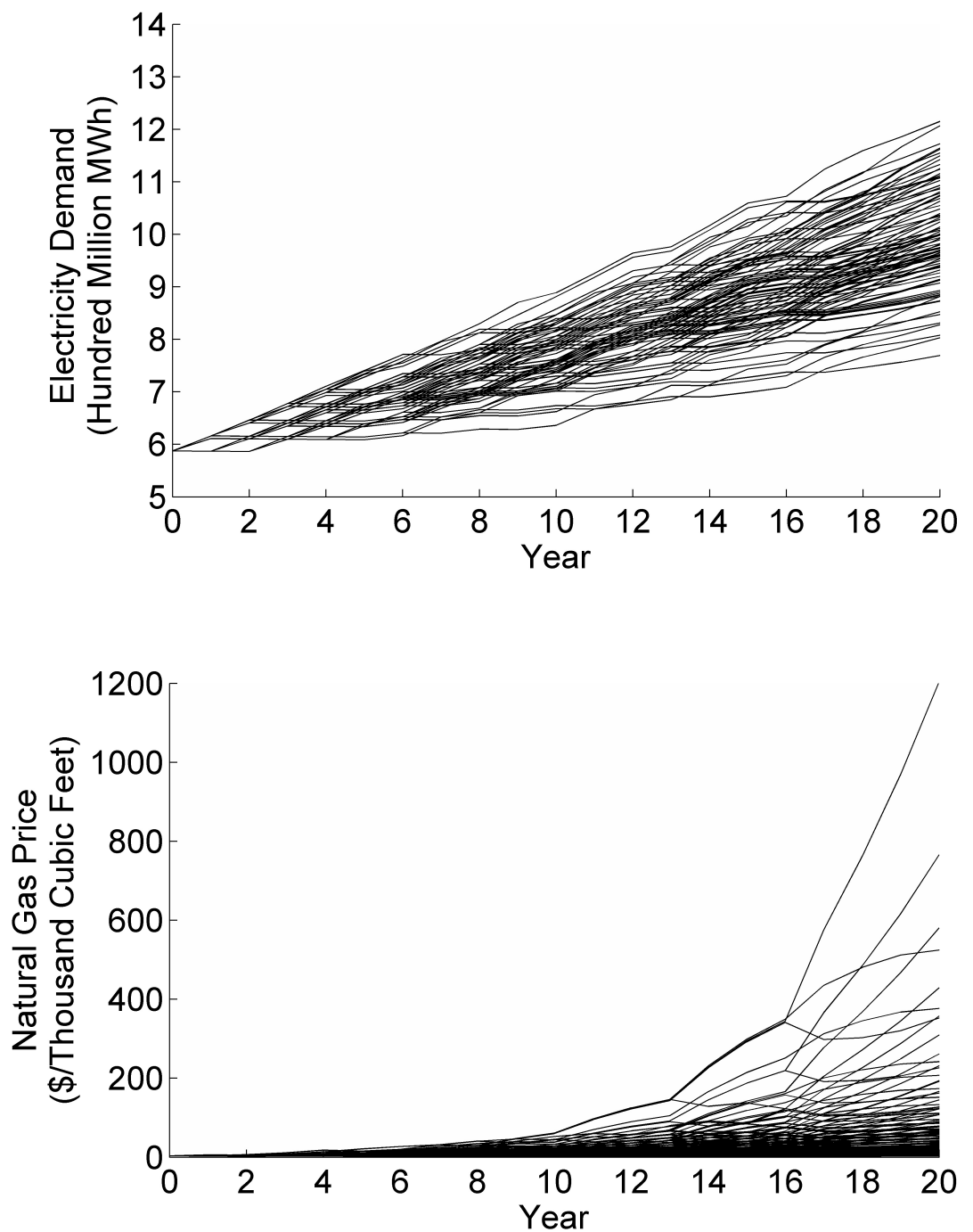

Figure 2.6 Scenarios selected by FFS method from the unequal-length scenario set (tree): (up) demand vs. time, (down) gas price vs. time 

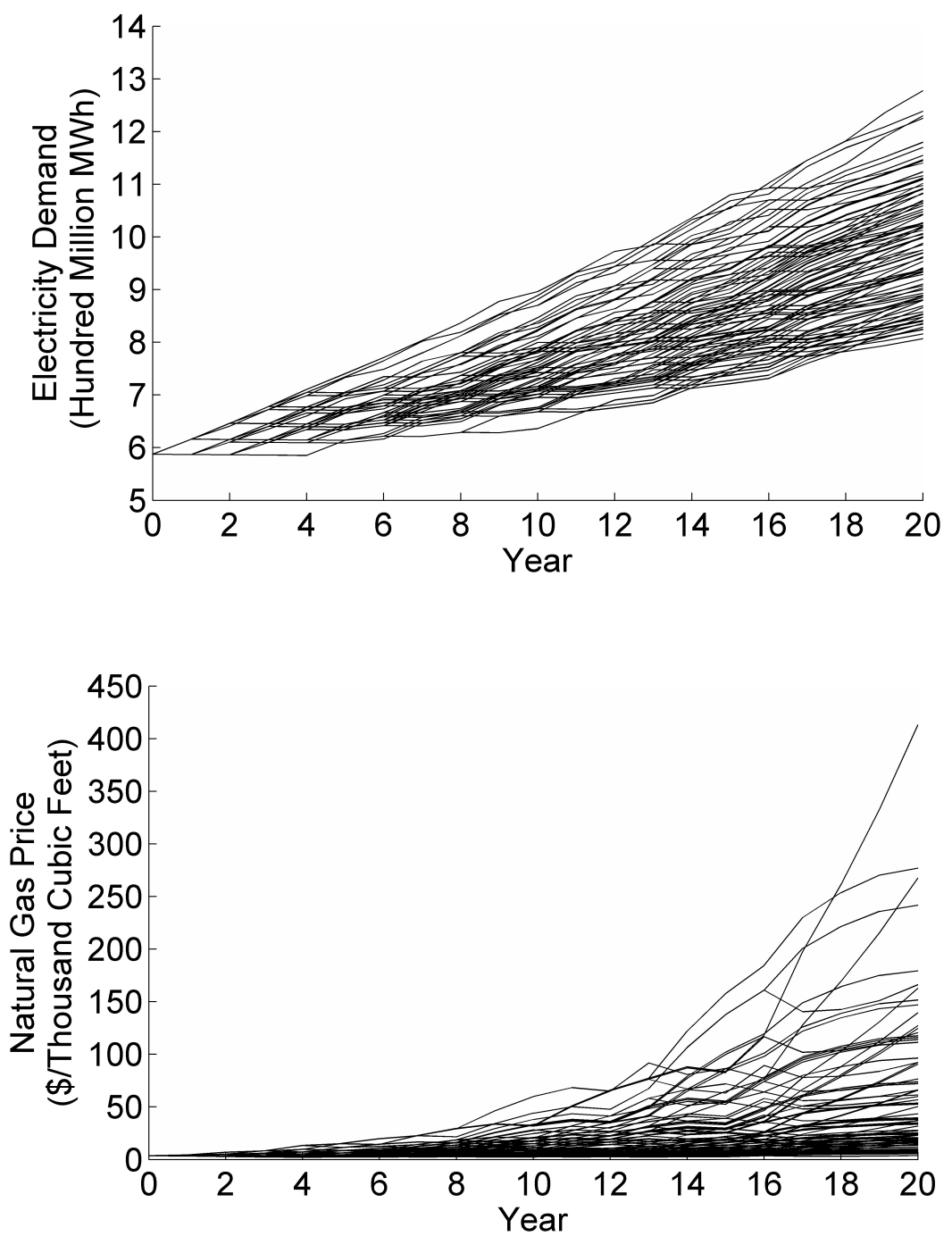

Figure 2.7 Scenarios selected by FSWC method from the unequal-length scenario set (tree): (up) demand vs. time, (down) gas price vs. time 

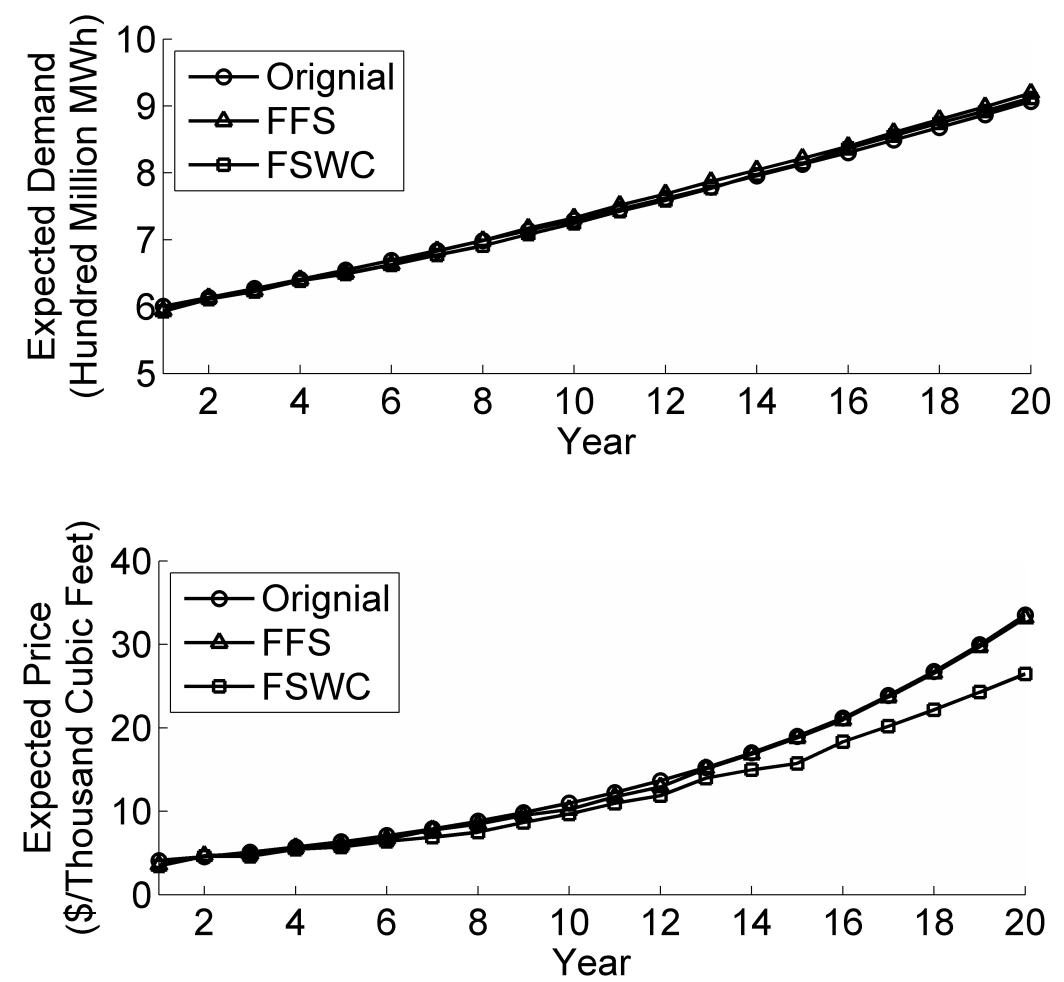

Figure 2.8 Comparisons among scenarios from original set, FSWC and FFS methods based on the unequal-length scenario set (tree): (up) expected annual electricity demand, (down) expected average natural gas price 
Table 2.8 The cumulative number of generators added based on FFS reduction of the unequal-length period tree

\begin{tabular}{l|r|r|r|r|r|r|r|r|r|r|r|r|r|r|r|r|r|r|r|r}
\hline \hline Year & 1 & 2 & 3 & 4 & 5 & 6 & 7 & 8 & 9 & 10 & 11 & 12 & 13 & 14 & 15 & 16 & 17 & 18 & 19 & 20 \\
\hline Base-load & 0 & 0 & 0 & 7 & 13 & 18 & 26 & 31 & 39 & 45 & 45 & 45 & 45 & 45 & 45 & 45 & 45 & 45 & 45 & 45 \\
Gas CC & 0 & 0 & 0 & 0 & 0 & 0 & 0 & 0 & 0 & 0 & 0 & 1 & 9 & 24 & 37 & 53 & 55 & 65 & 65 & 65 \\
Gas CT & 0 & 0 & 0 & 0 & 0 & 0 & 0 & 0 & 0 & 0 & 0 & 0 & 0 & 0 & 0 & 0 & 0 & 0 & 0 & 14 \\
Nuclear & 8 & 8 & 8 & 8 & 8 & 8 & 8 & 8 & 8 & 8 & 8 & 8 & 8 & 8 & 8 & 8 & 8 & 8 & 8 & 8 \\
Wind & 91 & 105 & 105 & 105 & 105 & 105 & 105 & 105 & 105 & 105 & 105 & 105 & 105 & 105 & 105 & 105 & 105 & 105 & 105 & 105 \\
IGCC & 13 & 45 & 45 & 45 & 45 & 45 & 45 & 45 & 45 & 45 & 45 & 45 & 45 & 45 & 45 & 45 & 45 & 45 & 45 & 45 \\
\hline
\end{tabular}

Table 2.9 The cumulative number of generators added based on FSWC reduction of the unequal-length period tree

\begin{tabular}{l|r|r|r|r|r|r|r|r|r|r|r|r|r|r|r|r|r|r|r|r|r}
\hline \hline Year & 1 & 2 & 3 & 4 & 5 & 6 & 7 & 8 & 9 & 10 & 11 & 12 & 13 & 14 & 15 & 16 & 17 & 18 & 19 & 20 \\
\hline Base-load & 0 & 0 & 0 & 7 & 13 & 15 & 20 & 25 & 32 & 39 & 45 & 45 & 45 & 45 & 45 & 45 & 45 & 45 & 45 & 45 \\
Gas CC & 0 & 0 & 0 & 0 & 0 & 0 & 0 & 0 & 0 & 0 & 0 & 0 & 12 & 17 & 31 & 43 & 55 & 65 & 65 & 65 \\
Gas CT & 0 & 0 & 0 & 0 & 0 & 0 & 0 & 0 & 0 & 0 & 0 & 0 & 0 & 0 & 0 & 0 & 0 & 0 & 23 & 23 \\
Nuclear & 8 & 8 & 8 & 8 & 8 & 8 & 8 & 8 & 8 & 8 & 8 & 8 & 8 & 8 & 8 & 8 & 8 & 8 & 8 & 8 \\
Wind & 105 & 105 & 105 & 105 & 105 & 105 & 105 & 105 & 105 & 105 & 105 & 105 & 105 & 105 & 105 & 105 & 105 & 105 & 105 & 105 \\
IGCC & 12 & 45 & 45 & 45 & 45 & 45 & 45 & 45 & 45 & 45 & 45 & 45 & 45 & 45 & 45 & 45 & 45 & 45 & 45 & 45 \\
\hline
\end{tabular}

show the optimal first-stage decisions based on each reduced set, illustrate that the expansion strategies based on the two scenario reduction methods have only minor differences.

Fig.2.9 shows the expected generation by generator type over the planning horizon from the solution to (2.1)-(2.5) based on the reduced scenario subsets obtained from FFS and FSWC, respectively, for the unequal-length period scenario tree. The figures show similar generation patterns result from applying reduced scenarios obtained from either scenario reduction method. IGCC generators start to increase generation from year 2, while the Base-load (coal) generators and those generators which consume natural gas decrease their generation at the same time. Generation from Base-load (coal) then increases after the fifth year. Fig.10 illustrates the expected generation costs resulting from the FFS- and FSWCbased expansion decisions for the unequal-length period tree.

According to Fig.2.10, the average generation costs of gas-fired CC and CT generators dramatically increase after the second year in each subplot, and from that year on, the generation costs of Baseload and IGCC generators are much lower than those of CC and CT generators. The potential for high natural gas prices later in the planning horizon favors energy production by coal-fueled Base-load and IGCC generators to minimize expected costs. The generation level of CC dramatically increases after the fifteenth year as the high electricity demand calls for more generators, and only CC and CT are available at that time.

Table 2.10 summarizes the investment cost, generation cost and penalty for unserved energy over the scenarios selected from the unequal-length period tree by the two reduction methods, respectively, 

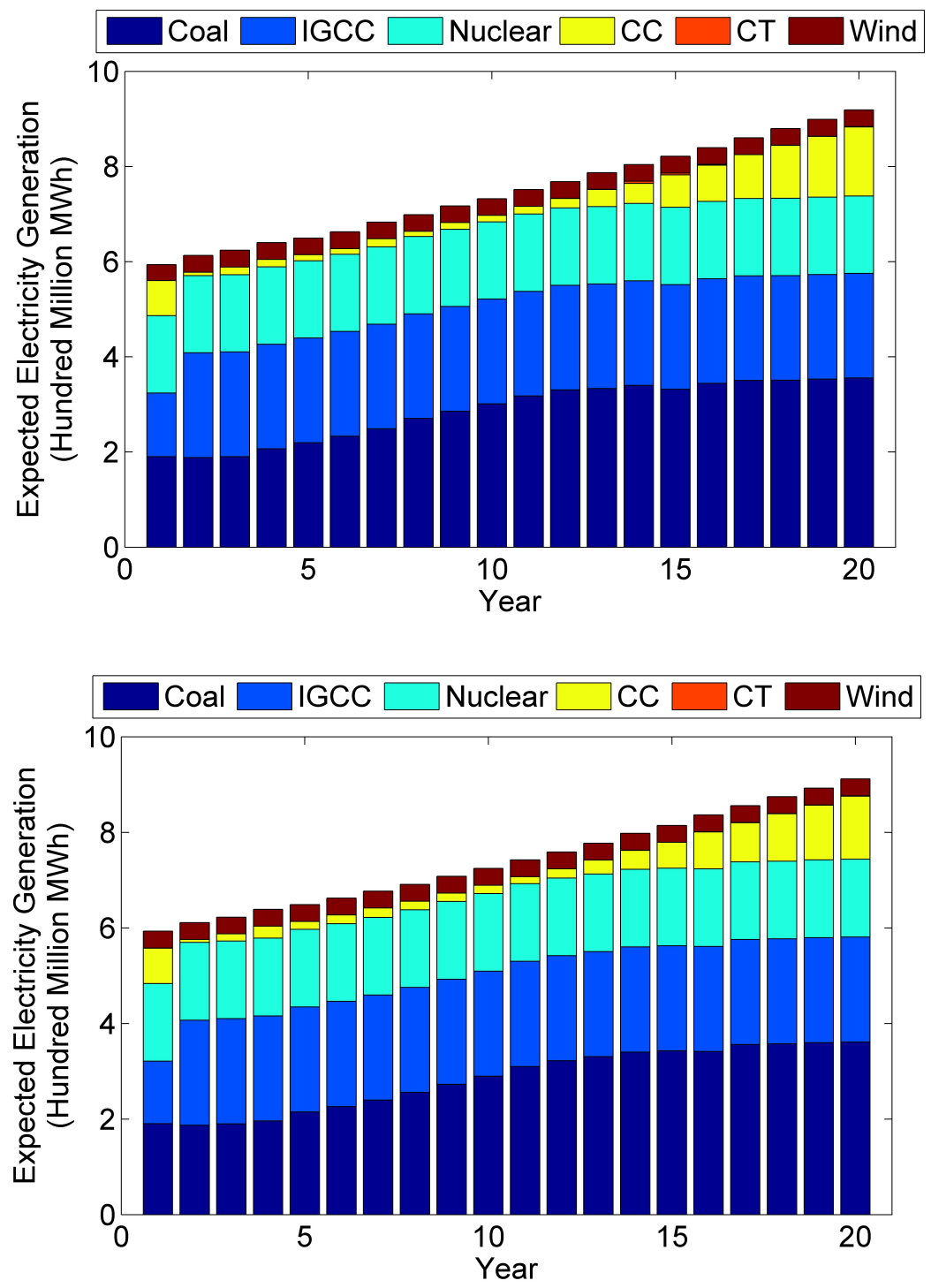

Figure 2.9 Illustration of expected energy generation of each type of generator based on the unequal-length period tree: (up) FFS method, (down) FSWC method 

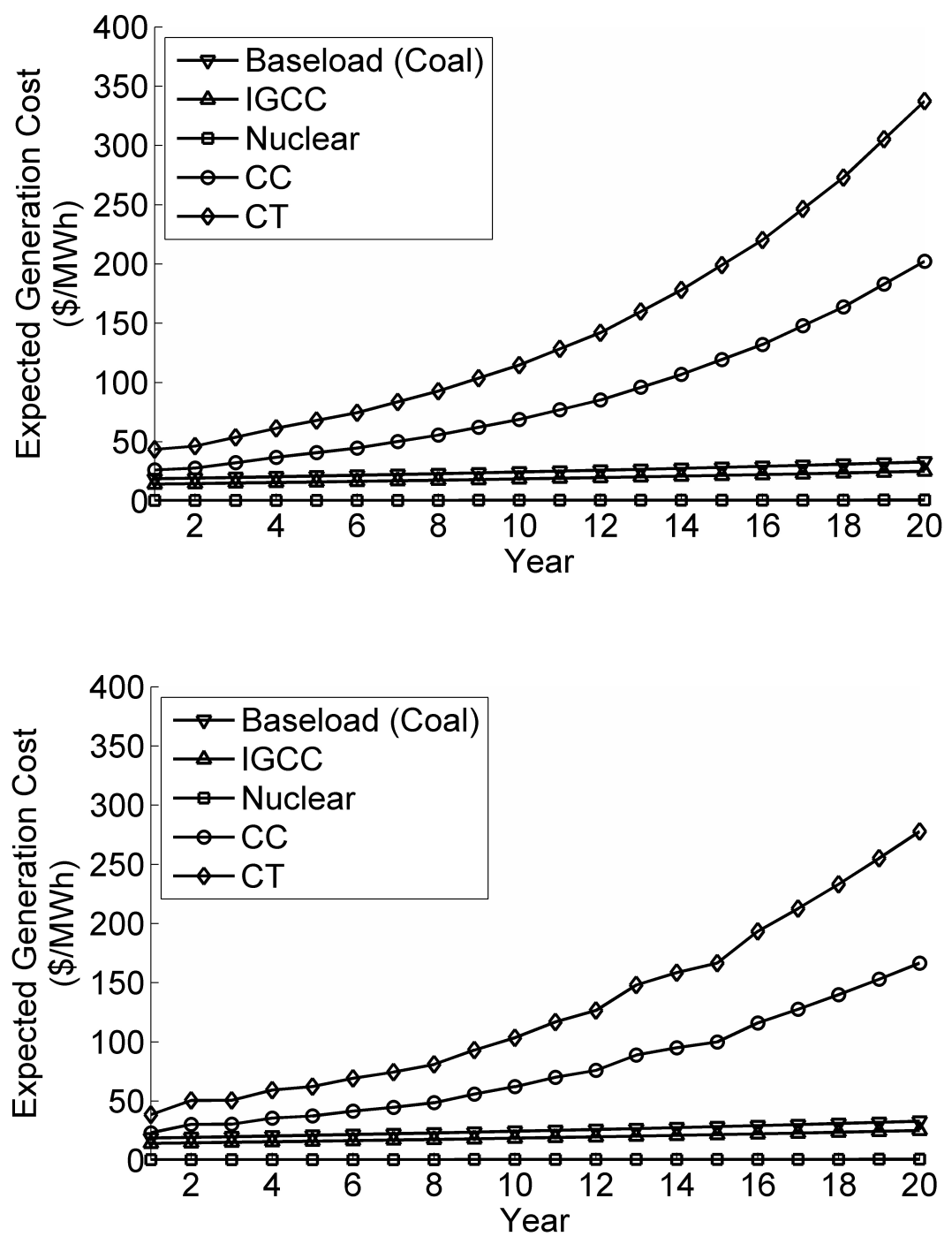

Figure 2.10 Expected generation cost of each type of generator over selected scenarios from the unequal-length period tree: (up) FFS method, (down) FSWC method 
and also illustrates the influences of expansion strategies on the original scenarios. Penalties occur when we solve the stochastic programm based on the selected scenarios derived from FSWC method. But it does not mean FSWC performs worse than FFS method does, because FSWC selected more extreme scenarios with higher electricity demand. Comparisons in Table 10 demonstrate that there are no major differences between FFS and FSWC in any cost category. The expected investment and generation costs from FSWC with respect to the selected scenarios are both smaller than those from FFS. One possible reason is that the generation costs of CC and CT from FSWC are lower than those from FFS, and no difference exists among the other types' generation costs, as Fig.10 illustrates. The lower costs from FSWC also derive from the cost discounting in objective function (1). The discount term $(1+r)^{(1-t)}$ indicates that the later the same type of generator is constructed, the lower the impact of investment cost for that type of generator. Therefore, despite the total number of Base-load and IGCC generators resulting from FFS and FSWC methods being similar, the discounted investment costs for the two types of generators are different. More CC and CT generators will be built in the strategy derived from FSWC, but because the building costs of Base-load and IGCC are much higher than those of CC and $\mathrm{CT}$, the total investment cost that results from the FFS method is still higher than that derived from FSWC method. The results of scenario reduction heuristics are further compared by fixing the firststage decisions according to the solution obtained with the reduced sets and then solving (2.1)-(2.5) as a continuous linear program using the original scenario tree. According to the expected penalty costs with respect to all scenarios, the expansion strategy obtained with the FSWC-reduced scenario subset can provide a more favorable outcome that not only leads to lower investment cost, but also to less unserved energy when the cardinality of the reduced scenario subset is greater than 10 .

The comparison of computational times for FFS and FSWC shown in Table 10 illustrates that FSWC may be more powerful than FFS in scenario reduction. The computational burden of FFS increases much more rapidly than the linear growth in $n$, the cardinality of the set of selected scenarios. Conversely, the computational times of FSWC may even decrease as $n$ increases. One possible reason is that selecting more scenarios in FSWC leads to a smaller size of each scenario cluster, and FFS must be applied only once in each cluster. Besides, the time spent on all pair-wise-distances comparison in each cluster is $O\left(N^{2}\right)$; thus, the smaller size of cluster may save much more time. 


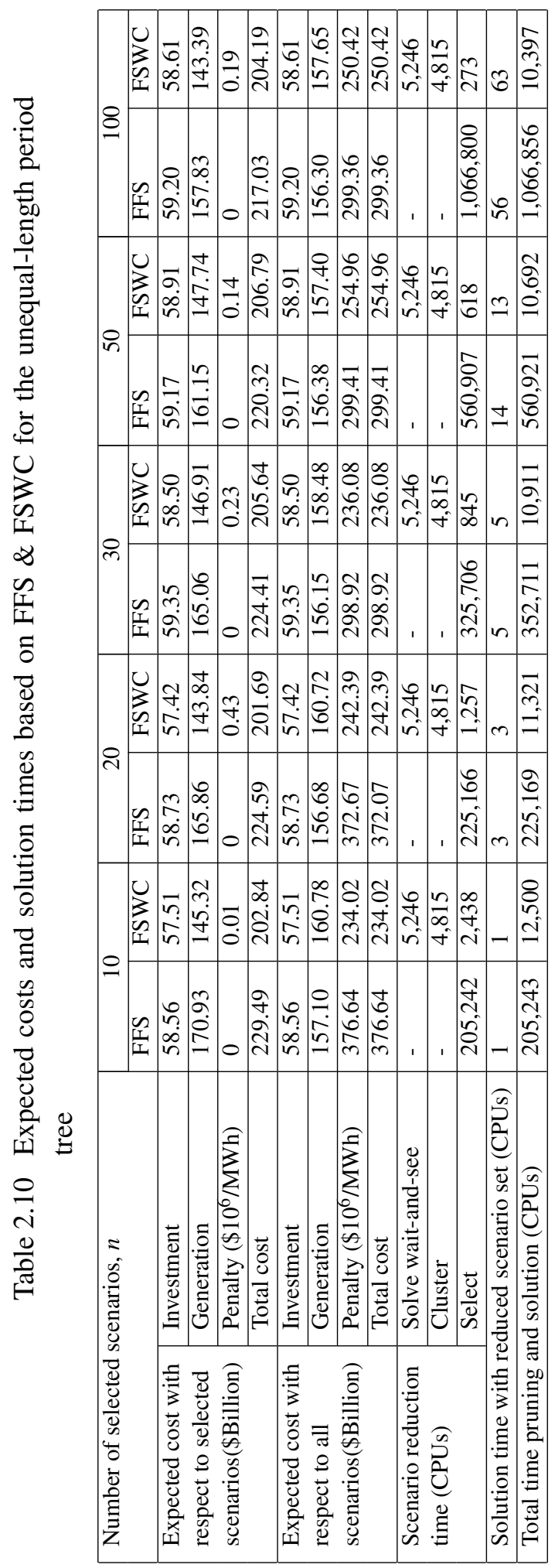


Table 2.11 The cumulative number of generators added based on FFS reduction of the equal-length period tree

\begin{tabular}{l|r|r|r|r|r|r|r|r|r|r|r|r|r|r|r|r|r|r|r|r}
\hline \hline Year & 1 & 2 & 3 & 4 & 5 & 6 & 7 & 8 & 9 & 10 & 11 & 12 & 13 & 14 & 15 & 16 & 17 & 18 & 19 & 20 \\
\hline Base-load & 0 & 0 & 3 & 8 & 14 & 20 & 26 & 32 & 39 & 45 & 45 & 45 & 45 & 45 & 45 & 45 & 45 & 45 & 45 & 45 \\
Gas CC & 0 & 0 & 0 & 0 & 0 & 0 & 0 & 0 & 0 & 0 & 0 & 10 & 22 & 35 & 48 & 62 & 65 & 65 & 65 & 65 \\
Gas CT & 0 & 0 & 0 & 0 & 0 & 0 & 0 & 0 & 0 & 0 & 0 & 0 & 0 & 0 & 0 & 0 & 0 & 0 & 20 & 49 \\
Nuclear & 8 & 8 & 8 & 8 & 8 & 8 & 8 & 8 & 8 & 8 & 8 & 8 & 8 & 8 & 8 & 8 & 8 & 8 & 8 & 8 \\
Wind & 100 & 105 & 105 & 105 & 105 & 105 & 105 & 105 & 105 & 105 & 105 & 105 & 105 & 105 & 105 & 105 & 105 & 105 & 105 & 105 \\
IGCC & 39 & 40 & 45 & 45 & 45 & 45 & 45 & 45 & 45 & 45 & 45 & 45 & 45 & 45 & 45 & 45 & 45 & 45 & 45 & 45 \\
\hline
\end{tabular}

Table 2.12 The cumulative number of generators added based on FSWC reduction of the equal-length period tree

\begin{tabular}{l|r|r|r|r|r|r|r|r|r|r|r|r|r|r|r|r|r|r|r|r|r}
\hline \hline Year & 1 & 2 & 3 & 4 & 5 & 6 & 7 & 8 & 9 & 10 & 11 & 12 & 13 & 14 & 15 & 16 & 17 & 18 & 19 & 20 \\
\hline Base-load & 0 & 0 & 2 & 8 & 9 & 10 & 16 & 22 & 29 & 34 & 41 & 45 & 45 & 45 & 45 & 45 & 45 & 45 & 45 & 45 \\
Gas CC & 0 & 0 & 0 & 0 & 0 & 0 & 0 & 0 & 0 & 0 & 0 & 0 & 22 & 35 & 49 & 62 & 65 & 65 & 65 & 65 \\
Gas CT & 0 & 0 & 0 & 0 & 0 & 0 & 0 & 0 & 0 & 0 & 0 & 0 & 0 & 0 & 0 & 0 & 0 & 0 & 20 & 49 \\
Nuclear & 8 & 8 & 8 & 8 & 8 & 8 & 8 & 8 & 8 & 8 & 8 & 8 & 8 & 8 & 8 & 8 & 8 & 8 & 8 & 8 \\
Wind & 93 & 105 & 105 & 105 & 105 & 105 & 105 & 105 & 105 & 105 & 105 & 105 & 105 & 105 & 105 & 105 & 105 & 105 & 105 & 105 \\
IGCC & 11 & 19 & 45 & 45 & 45 & 45 & 45 & 45 & 45 & 45 & 45 & 45 & 45 & 45 & 45 & 45 & 45 & 45 & 45 & 45 \\
\hline
\end{tabular}

\subsubsection{Numerical results for the equal-length period lattice}

The corresponding numerical results for the second scenario set are provided in this section. As for the first scenario set, there are minor differences between scenarios selected by the two reduction methods with respect to annual electricity demand, but larger differences in terms of the natural gas prices (Fig.2.11 and Fig.2.12). As Fig.2.13(up) shows, comparing to the scenarios selected by FFS, the expected annual electricity demand of the scenarios selected by FSWC is closer to the expected level based on the original scenarios. Similar to the numerical results in Section 2.6.5.1, the expected natural gas prices of scenarios selected by FFS are closer to the original scenarios, and the same explanation of the phenomenon applies.

Again, GEP model (2.1)-(2.5) was solved to obtain the optimal first-stage decisions to assess the performance of solutions based on the reduced scenario sets. Tables 2.11 and 2.12, which show the optimal first-stage decisions based on each reduced set from the equal-length period lattice, illustrate that the expansion strategies based on the two scenario reduction methods have only minor differences.

Like Fig.2.9, Fig.2.14 shows the expected generation by generator type over the planning horizon obtained by solving model (2.1)-(2.5) based on the reduced scenario subsets obtained from FFS and FSWC, respectively, for the equal-length period lattice. The figures show similar generation pattern$\mathrm{s}$ result from applying reduced scenarios obtained from either scenario reduction method. Similar to Fig.2.9, IGCC and Base-load generators start to increase their generation from year 2 and year 3 re- 

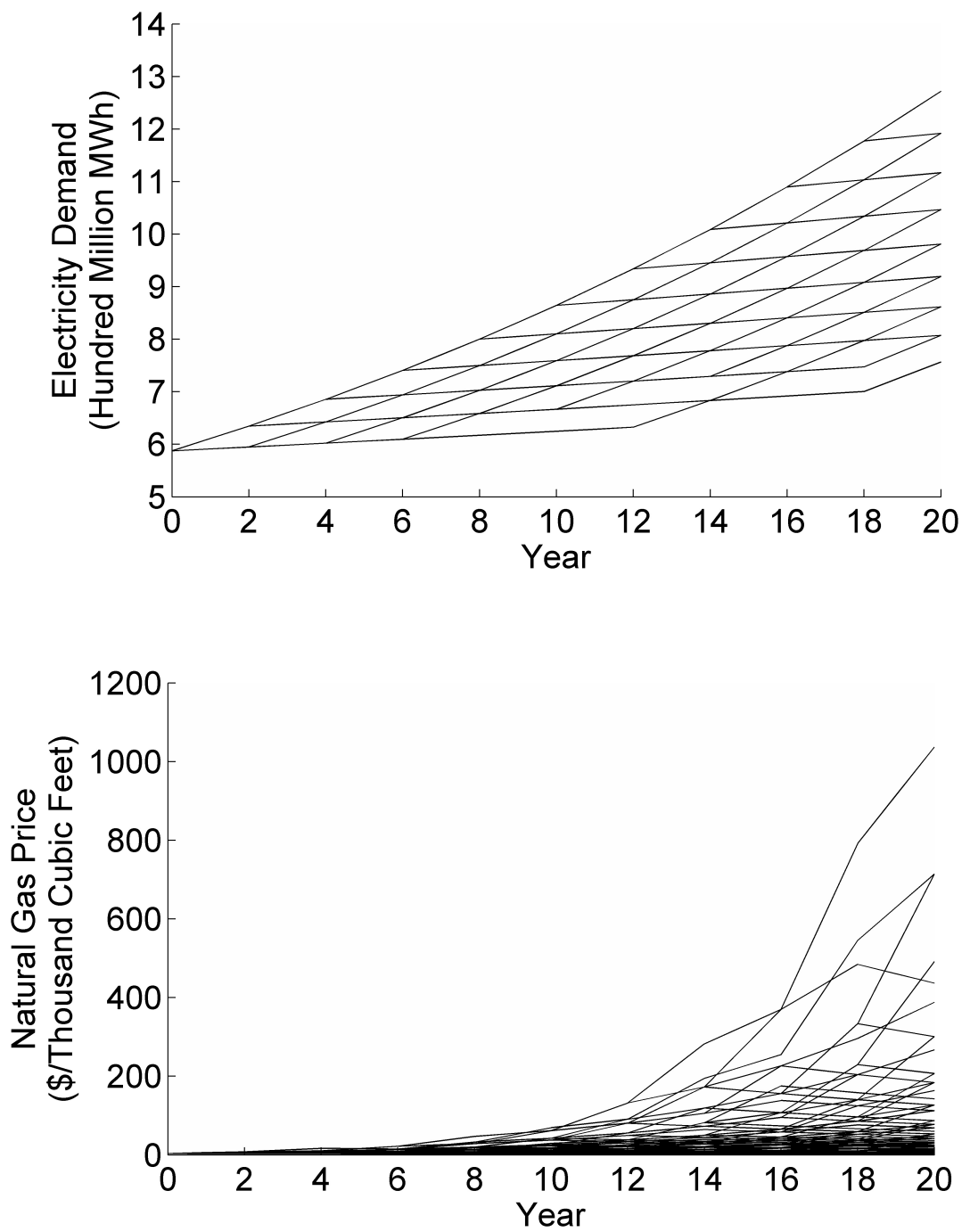

Figure 2.11 Scenarios selected by FFS method from the equal-length period lattice: (up) demand vs. time, (down) gas price vs. time 

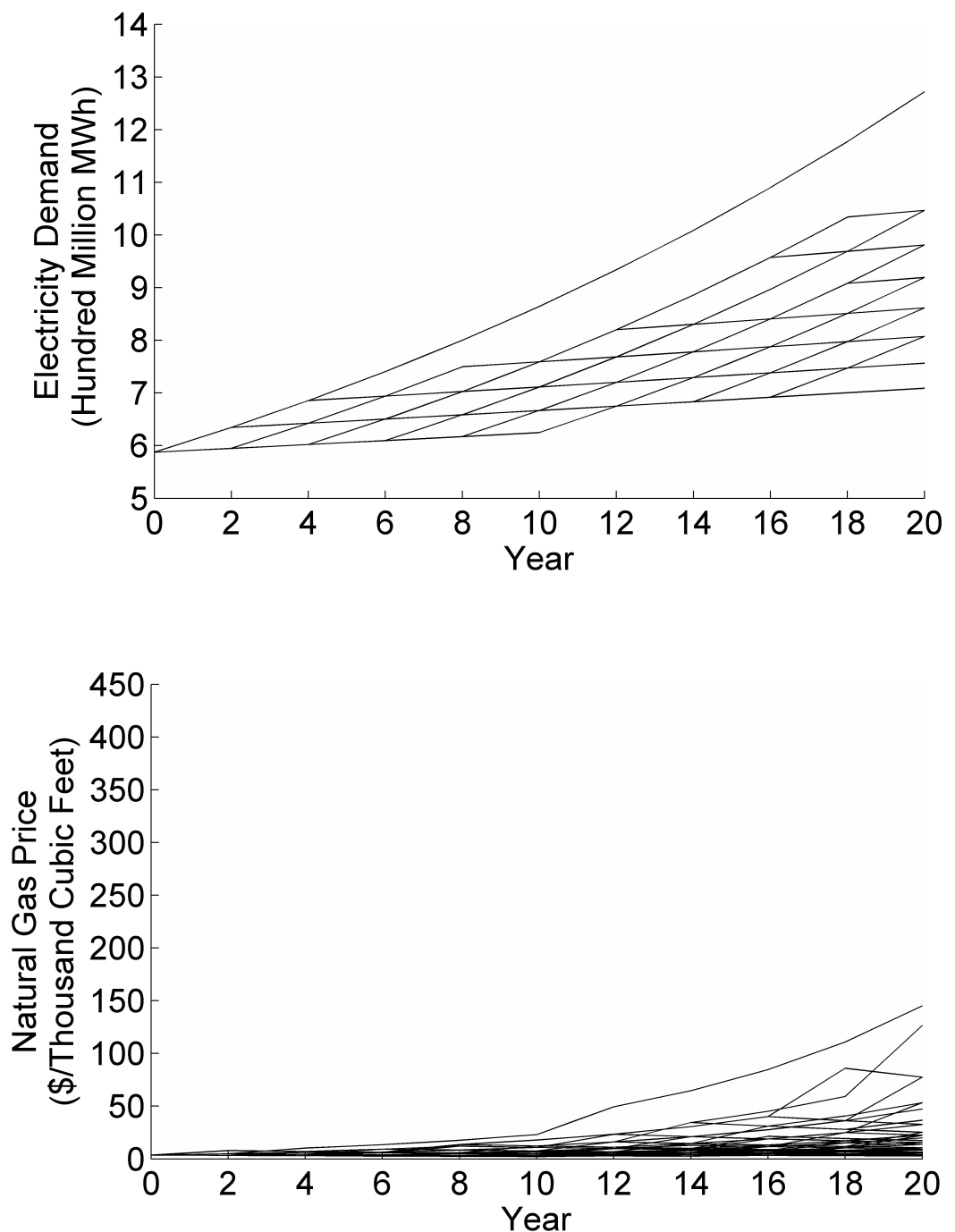

Figure 2.12 Scenarios selected by FSWC method from the equal-length period lattice: (up) demand vs. time, (down) gas price vs. time 

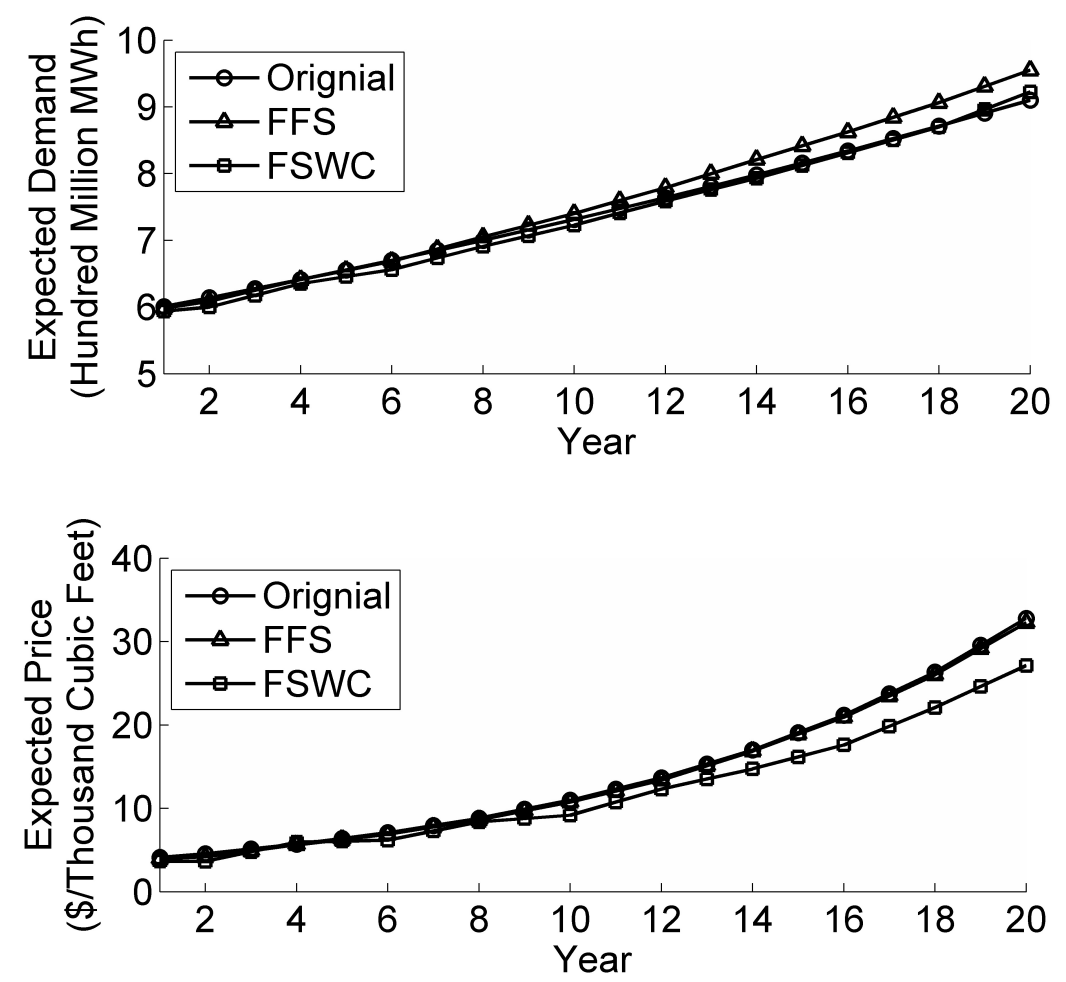

Figure 2.13 Comparison of average electricity demand and natural gas price between two methods based on the equal-length period lattice: (up) expected annual electricity demand, (down) expected average natural gas price 
spectively, while the generators which consume natural gas decrease their generation at the same time in equal-length period lattice. Fig.2.15 illustrates the expected generation costs resulting from the FFSand FSWC-based expansion decisions for the equal-length period lattice.
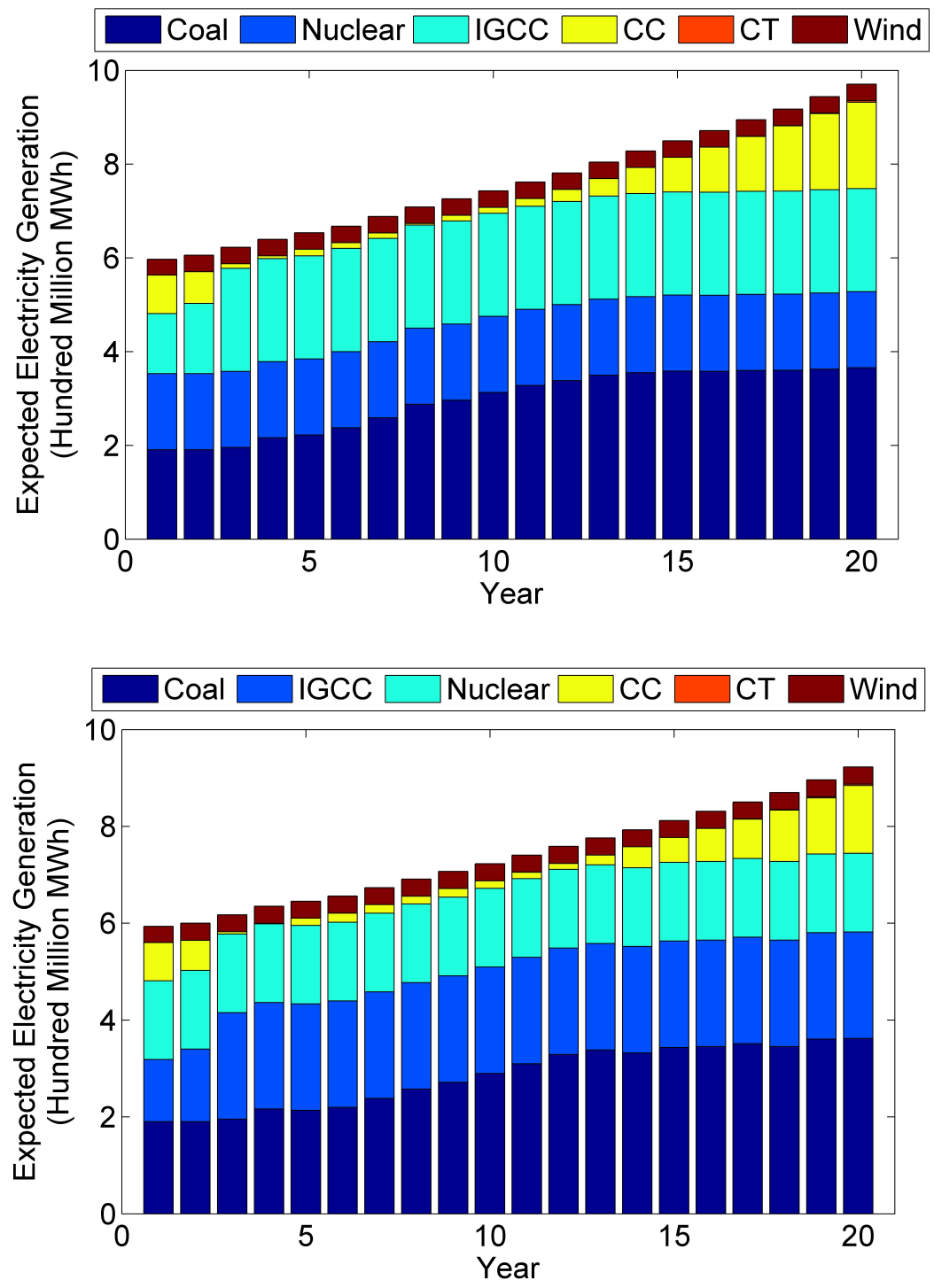

Figure 2.14 Illustration of expected energy generation of each type of generator based on the equal-length period lattice: (up) FFS method, (down) FSWC method

Similar to the pattern shown in Fig.2.10, the average generation costs of gas-fired CC and CT generators dramatically increase after the second year in each subplot of Fig.2.15, and from that year on, the generation costs of Base-load and IGCC generators are much lower than those of CC and CT 

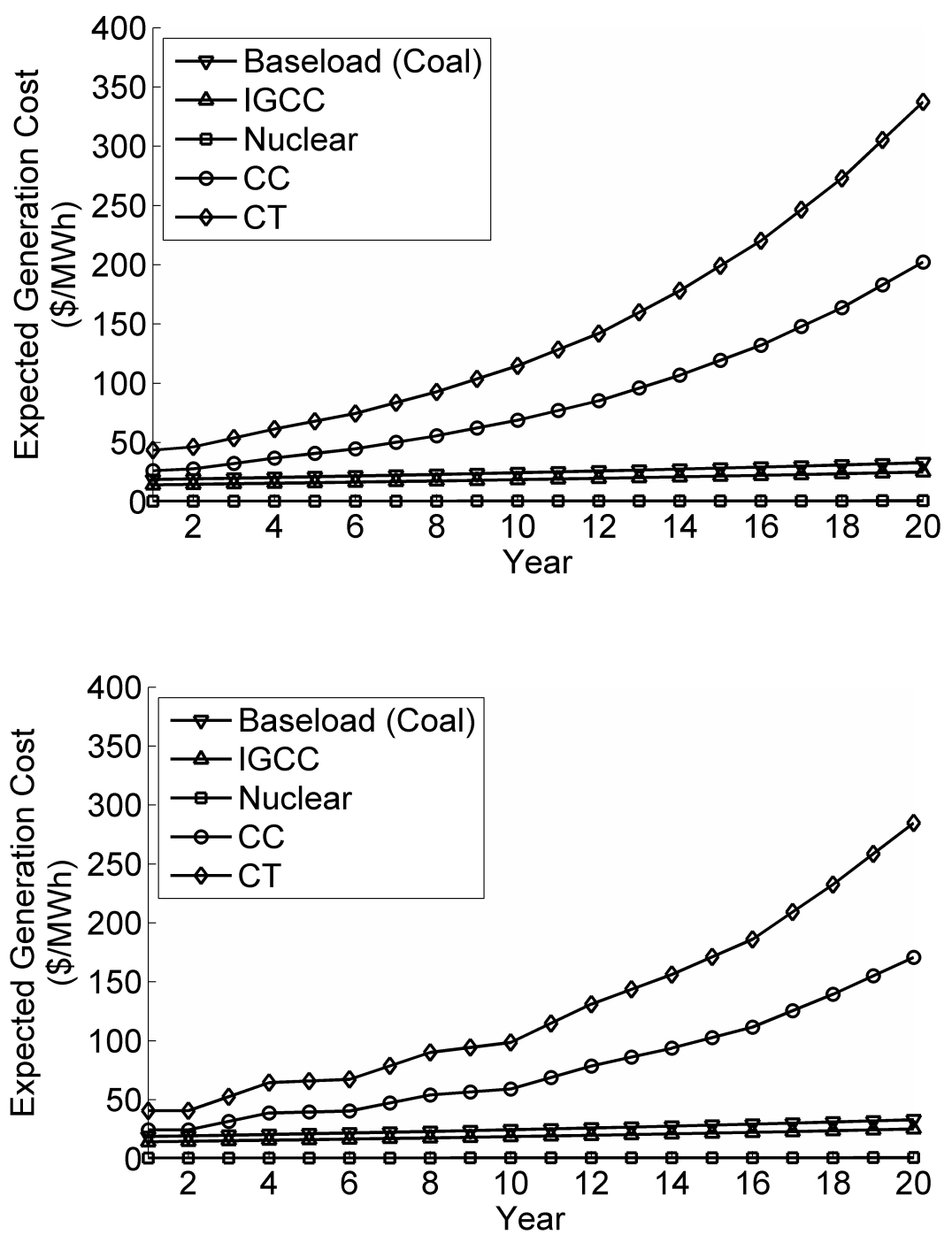

Figure 2.15 Expected generation cost of each type of generator over selected scenarios from the equal-length period lattice: (up) FFS method, (down) FSWC method 
generators. The potential for high natural gas prices later in the planning horizon leads to more energy production by Base-load and IGCC generators to minimize expected costs.

Like Table 2.10, Table 2.13 summarizes the investment cost, generation cost and penalty for unserved energy based on scenarios selected from the equal-length period lattice by two reduction methods respectively, and also illustrates the performance of the expansion strategies with respect to the original scenarios. For the equal-length period lattice, FSWC and FFS methods provide similar results in every cost category, especially when the reduced scenario subset cardinality is set to 20,50 or 100 . Since any unserved energy will be penalized by $10^{7} \$ / M W h$, the penalties in expected cost with respect to all scenarios indicate that only a small amount of demand would be unsatisfied over the planning horizon.

Considering the computational times summarized in Tables 10 and 13, FFS is impractical for selecting modest scenario subsets from large scenario sets. In contrast to the unsatisfactory performance of FFS for these large scenario sets, FSWC requires computational times ranging from $15 \%$ of that for FFS when selecting 10 scenarios to less than $1 \%$ when selecting 100 scenarios. But it results in similar first-stage decisions and expected costs with respect to the original scenario sets. Moreover, the time required for FSWC is approximately constant over different reduced set cardinalities.

\subsection{Conclusions}

This chapter provides an approach to generating scenario trees to cover a long time horizon with variable length periods under the assumption that the random quantities follow correlated GBM processes. The cardinalities of the generated scenario sets are controlled both by combining multiple years into periods of varying lengths and by matching the number of branches from each node to the degrees of freedom in the statistical specifications. The FSWC scenario reduction heuristic applied in this chapter clusters scenarios based on their wait-and-see solutions and then applies the fast forward selection heuristic within clusters. Thus, it incorporates the stochastic optimization context in addition to the probability distribution of scenarios. The numerical results obtained from a simplified generation expansion planning model indicate the usefulness and efficiency of the FSWC method. The first-stage solutions obtained from the FSWC-reduced scenarios are similar to those obtained from the 


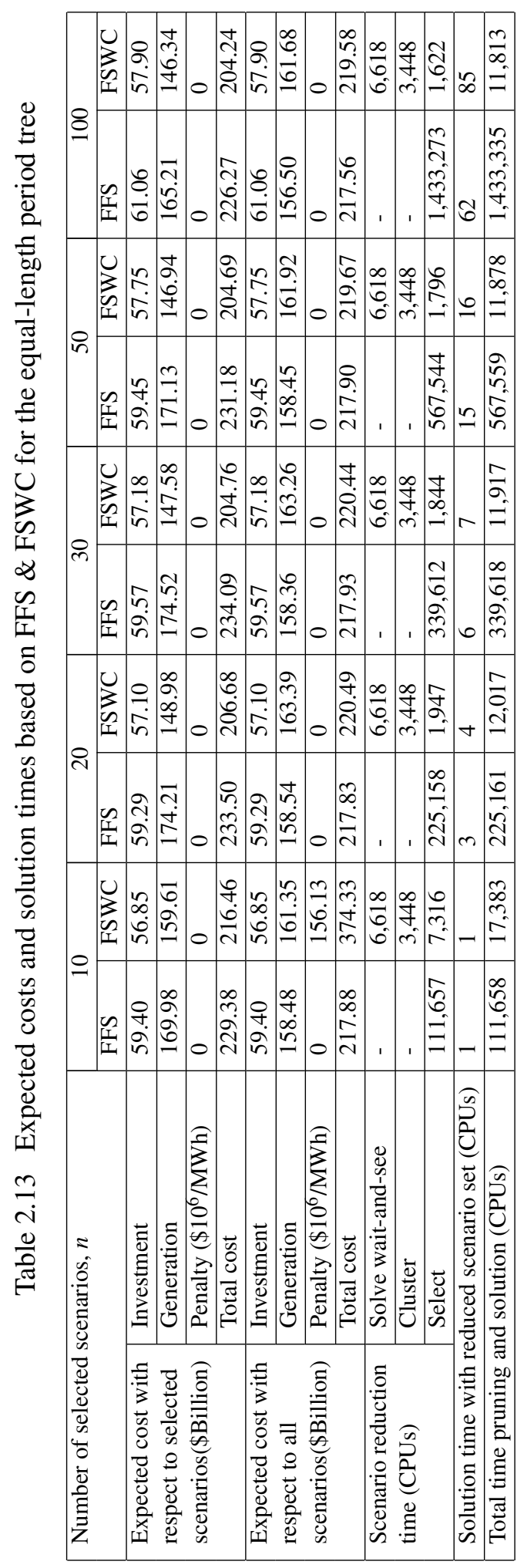


FFS-reduced scenarios, as are their expected costs when implemented on the whole set of scenarios. But the computational time for FSWC is substantially smaller and does not vary significantly with the cardinality of the reduced set.

In this chapter, risk associated with generation expansion decisions has been incorporated by including a penalty for unserved energy but only in terms of expectation. Some simplifying assumptions made in this chapter are relaxed and risk is included in terms of conditional value at risk Feng and Ryan (2012). Because the success of FSWC depends on choosing appropriate key first-stage variables on which to cluster, guidance for their selection in various applications is a significant research issue. 


\title{
CHAPTER 3. DAY-AHEAD HOURLY ELECTRICITY LOAD MODELING BY FUNCTIONAL REGRESSION
}

\author{
A paper prepared to submit to Applied Energy
}

Yonghan Feng and Sarah M. Ryan

\begin{abstract}
Short-term load forecasting is important for power system generation planning and operation. As the attention on economic and reliable unit commitment with uncertain load and variable generation increases, it is necessary to establish a short-term load model that not only provides accurate load predictions but also helps to generate reasonable probabilistic scenarios or ranges to be represented in uncertainty sets. This paper proposes a fundamental load incorporated weather conditional epispline load model (FWEp), which is based on weather forecast and function approximation. Load data transformations are first implemented between various day types and a specified reference day type in similar weather days in the same season in the same area, in order to capture the fundamental load pattern through enriched data. Instead of using common linear regression methods, FWEp fits functions from time to load, and from weather to load by approximating their second derivatives as piece-wise constant functions, separately in each group of similar weather days in each season and each geographical area. In an instance derived from an Independent System Operator in the U.S., FWEp not only provides accurate hourly load prediction and narrow band of prediction errors in the test set, but also preserves similar intraday serial correlations among hourly load values to those of actual hourly load.
\end{abstract}




\subsection{Introduction}

Short-term load forecasting is one of the significant tasks in operations of power systems. The forecasted load influences unit commitment, economic dispatch, maintenance plans for generators and electricity price forecasting in power markets. As emphasis on efficient and robust scheduling of thermal generators with uncertain load prediction increases, stochastic programming and robust optimization, have been extensively studied for use the operations of power systems (Takriti et al., 1996; Wu et al., 2007; Ruiz et al., 2009; Cheung et al., 2014; Bertsimas et al., 2013; Jiang et al., 2012; Guan and Wang, 2014; Chen et al., 2014). In the context of stochastic/robust unit commitment or economic dispatch, it is necessary to identify an appropriate short-term load model which provides not only an accurate hour-by-hour point forecast but also appropriate unbiased forecasting intervals to generate reasonable probabilistic scenarios or ranges with which to form uncertainty sets.

Various approaches have been proposed over the past decades to model or forecast short-term load. Most of the short-term load modeling approaches can be categorized as statistical methods or artificial intelligence (AI) based methods. The former often presume that load is a function of several explanatory variables; e.g., previous load values, temperature and humidity, and then estimate parameters of the specified function. In contrast, AI methods do not restrict themselves to specified functions. The AI methods include artificial neural networks (Fan et al., 2009; Chen et al., 2010), support vector machines (Mohandes, 2002; Fan and Chen, 2006; Wang et al., 2011; Che and Wang, 2014), and other techniques. See Feinberg and Genethliou (2005); Kyriakides and Polycarpou (2007); Hong (2011) for broad reviews of AI methods. Similar days methods also used by independent system operator and utilities to forecast future load and wind energy (Mahoney et al., 2012; ISO New England, 2013a). In these methods, similar days are often defined to be those historical days with similar weather and day type, and the actual load of a similar day is taken as a forecast. Similar days methods are often embedded in a more complicated load model, as in Chen et al. (2010), because the methods are not sufficient in themselves to capture complex load features if used alone.

Among statistical methods, time series and regression methods are widely used to build short-term load models. The autoregressive moving average (ARMA) model is one of the most used time series methods (Amjady, 2001; Huang and Shih, 2003; Taylor, 2003; Liu et al., 2006; Taylor, 2010). Amjady 
(2001) identified different ARMA models for hot days and cold days while considering effects from weekdays and weekends on daily load patterns. Huang and Shih (2003) presented a modified ARMA short-term model by considering non-Gaussian process. Taylor (2003) established a univariate time series load model which considered within-day and within-week seasonalities by using exponential smoothing. His work is further improved by accounting for triple seasonalities, the intraday, intraweek and intrayear effects, of short-term load in Britain and France (Taylor, 2010). Liu et al. (2006) improved upon the previous work by estimating the effects of temperature, hour-of-day and type-of-day on load level using nonparametric regression, and employed an ARMA model to capture the serial correlation in the load data. In general, time series methods predict well for the immediate future, but they may suffer from prediction precision issues for the multi-step ahead predictions because of the accumulated prediction errors.

Regression on various explanatory variables is another major direction of short-term load modeling. Usually, regression methods model a functional relationship between load and weather conditions, day types, holidays, economic conditions, and social habits of individuals. Papalexopoulos and Hesterberg (1990) and Engle et al. (1992) considered impacts of temperature and holidays on load. Haida and Muto (1994) applied transformations to include load changes in recent days and seasons, as well as annual load growth in a linear regression model. Charytoniuk et al. (1998) considered load forecast as a local average of observed past loads within the local neighborhood and the specific weights on the loads defined by a multivariate product kernel. Hong (2011) presented a multiple linear regression model that considered load as a polynomial function of temperature for each hour in each day type in each month. Black (2011) also applied multiple linear regression to examine the influence of weather on load, but focused on summer weekdays in the region served by ISO-New England. Charlton and Singleton (2014) refined the multiple linear regression model of Hong (2011), and achieved small prediction errors and weighted RMSE simultaneously in 2012 Global Energy Forecasting Competition, by taking into account multiple weather stations, day-of-season effects and smoother temperature forecasts. Fan and Hyndman (2012) proposed a semi-parametric additive model, which applied cubic splines to estimate relationship between load and temperatures, as well as effects from previous loads. Most of the multiple regression methods mentioned here achieved encouraging point predictions by establishing a load model for each hour (or each half hour) in a day, but with the exception of (Fan and Hyndman, 2012), did 
not take into account or assess forecast distribution for each hour. In order to generate realistic load scenarios, the proposed load model in our paper aims to provide not only accurate hourly load prediction but also less biased distribution of prediction errors, by extending and improving the load model in (Feng et al., 2013) which predicts the trajectory of hourly load in a group of similar days in each season and area.

Although many load backcasting studies that have presented encouraging matches to actual loads using actual weather records as input, the associated load models may not be satisfactory for constructing probabilistic load scenarios or uncertainty sets for day-ahead unit commitment. Even if the model accurately captures the effects of weather on electricity demand, the day-ahead weather forecast is still imperfect. In this paper we base the day-ahead load model that on the day-ahead weather forecast that is available to planners so that load forecast error distributions reflect the imprecision in both weather forecasting and modeling the relationship between weather and load. The contributions of this paper include the development of a short-term load model based on weather forecast and function approximation, which not only succeeds to accurately predict hourly load by modeling the complex nonlinearity between weather (i.e. temperature and dew point temperature) and electricity consumption level in multiple geographic zones, but also achieves similar intraday serial correlations among hourly load values to those of actual hourly load, as well as narrower and less biased prediction errors. In an case study derived from the Independent System Operator of New England (ISO-NE), the proposed short-term load model leads to a narrower and less biased distribution of prediction errors for each zone in each season, compared to two recent regression based short-term load models (Hong, 2011; Charlton and Singleton, 2014).

The rest of the paper is organized as follows. Section 3.2 partitions data into several segments according to calendar effects (day types and seasons), and enriches the data by transformation to a reference day type. Section 3.3 develops two weather conditional short-term load models by applying epi-spline approximation techniques. Section 3.4 compares performances of the two epi-spline based short-term load models over the data in each zone of ISO-NE in the test set, and shows that fundamental load pattern is another significant factor in short-term load model, in addition to the weather conditions. Moreover, Section 3.4 shows that our model succeeds in capturing the complex nonlinear relationship between electricity consumption level and weather conditions, and to attain narrower and less biased 
distributions of prediction errors, compared to those of two benchmark models. Section 3.5 concludes this paper. As supplementary and extension material to the journal paper draft, several remarks about scenario generation are presented in Section 3.6. Appendices to the paper, included in Sections 3.7 and 3.8 of this chapter, provide the relevant deductions of one-dimensional epi-splines and possible extensions to multiple factors.

\subsection{Data processing}

In this paper, a day-ahead hourly load model for 8 zones in ISO-NE is developed based on their corresponding weather forecasts. Although historical data back to 2003 can be found on the website of ISO-NE, we developed the load model based on historical load and weather forecast data which ranged from April 2009 to March 2011, and validated the model performance on data covering April 2011 March 2012. Because the relationships between load and predictor variables, e.g. weather conditions, are gradually changing over years.

\subsubsection{Division of seasons}

The relationship between weather forecast and electricity consumption changes during the year because of heating in winter versus cooling in summer, as well as diurnal lighting patterns. From the scatter plots of electricity consumption level vs. temperature forecast (Fig. 3.1) in zone CT which share the most, about $25 \%$, of electricity consumption in ISO-NE, it appears that electricity consumption will quickly increase as temperature rises in summer months (i.e., June through September), while decreasing with temperature in the winter. As discussed in Amjady (2001); Liu et al. (2006); Hong (2011), the accuracy of load prediction may be improved if calendar effects are considered in a load model. In this paper, calendar months with similar linear trends, of electricity consumption based on temperature are grouped into the same season. Although electricity consumption may be more accurately captured by a nonlinear function of temperature in each month, the linear trends appear sufficient for clustering similar months. The linear trend for each month is fitted by using simple linear regression of the actual load to the forecast temperature in the training set, which ranges from April, 2009, to March, 2011, for each zone in ISO-NE. Three seasons, winter, summer, and spring- 
fall (artificially combines April, May, October and November), are then created by clustering months according to their linear trends. The clustering is implemented by applying the $k$-means method to the slopes of the regression lines.
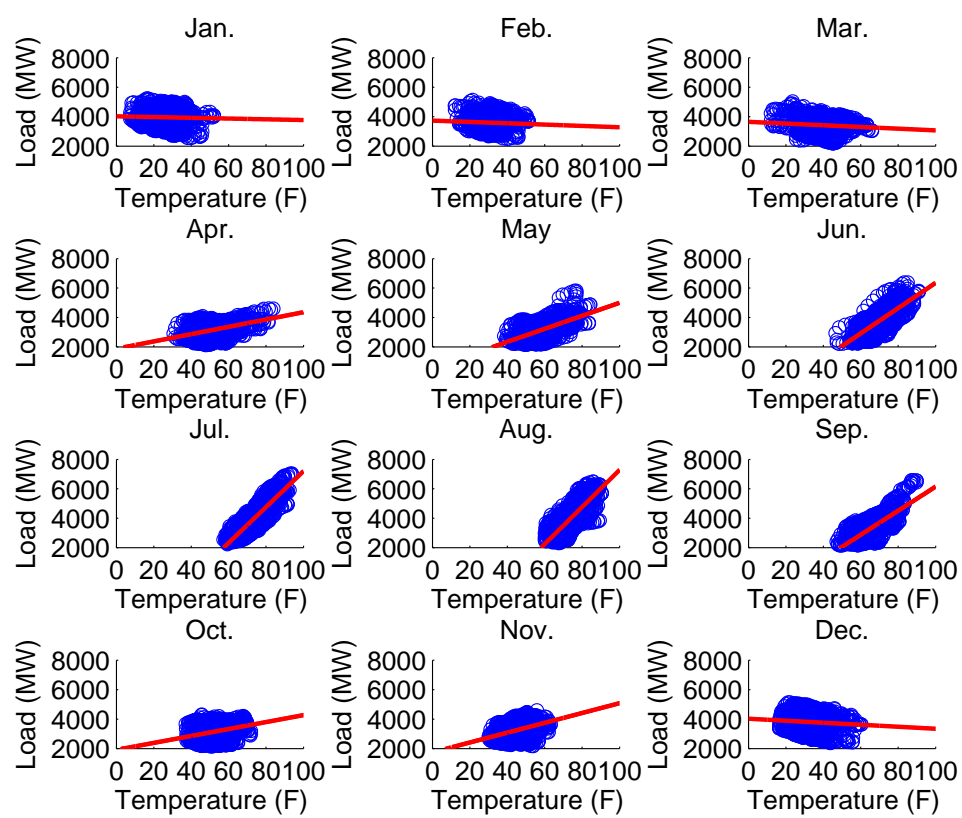

Figure 3.1 Scatter plot of load vs. temperature for each month in the training set, CT (Apr. 2009 - Mar. 2011)

\subsubsection{Days segmentation}

A short-term load model can be established for each specified season. However, Fig. 3.2 reveals that the 24-hour temperature vector of each day varies in a wide range even in the same season. The forecasting accuracy of a load model could be improved if it is specified separately in each range of temperatures. In this paper, days in a season are divided into 3 segments: low, moderate, and high temperature days, by applying $k$-means clustering to the mean temperature of each day. Clustering on 24-hour temperature vectors would be another way to group days in a season, but it is difficult to perform clustering on high dimensional vectors. Before proceeding to the mathematical expression of a short-term load model, a transformation process is introduced in Section 3.2.3 to enrich the data in each 
segment in a season.

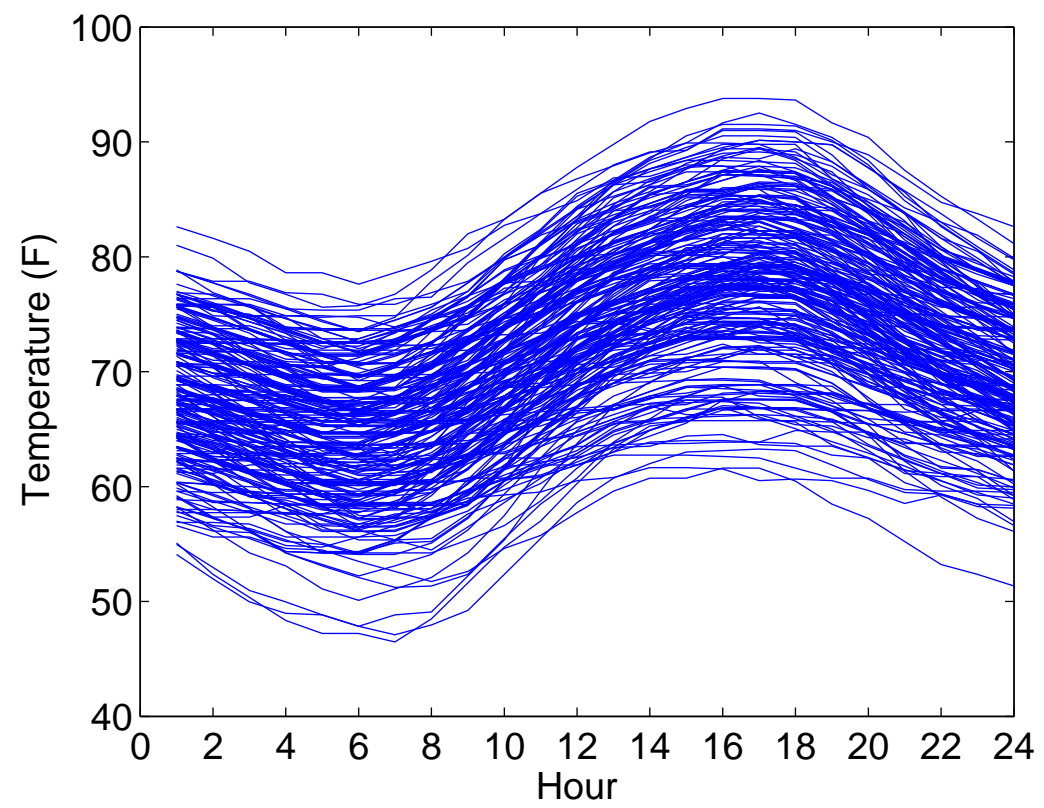

Figure 3.2 24-hours temperature through summers in the training set, CT (Apr. 2009 - Mar. 2011)

\subsubsection{Load data transformation}

Many short-term load model approaches identify an hourly load model for each day type, as in (Amjady, 2001; Liu et al., 2006; Hong, 2011; Fan and Hyndman, 2012). However, this requires a large amount of historical data. On the other hand, for a reliable prediction model, we wish to use only recent data to reflect current economic and demographic conditions. Average hourly load sequence for each day of the week are plotted for each season in Fig. 3.3, which shows that the pattern of the load sequence in each day type in a week in the same season tends to be similar, although the load levels in weekends (Saturday and Sunday) are lower than those from weekdays. Based on this similarity in load shapes, we enrich the data in each segment by applying a transformation among day types for each season. The rest of Section 3.2.3 presents the data transformation procedure briefly mentioned in Feng et al. (2014).

Let $\mathscr{D}$ be the set of days of a specified day type in a segment within a season, and $\mathscr{D}^{\prime}$ be the set of days of another day type in the same segment of the same season. Suppose $t_{\omega} \in \mathbb{R}^{24}$ is a sequence of 
hourly temperature forecast values in a day $\omega \in \mathscr{D}$, and let $\psi_{o}: \mathbb{R}^{24} \rightarrow \mathbb{R}^{24}$ be an appropriate operator that maps $t_{\omega}$ (or in addition to time periods) to the corresponding sequence of hourly load $l_{\omega} \in \mathbb{R}^{24}$ :

$$
l_{\omega}=\psi_{o}\left(t_{\omega}\right)+e_{\omega}, \forall \omega \in \mathscr{D}
$$

so that $\left\|e_{\omega}\right\|$ be minimized over $\mathscr{D}$. Then

$$
\tilde{l}_{\omega^{\prime}}=\psi_{o}\left(t_{\omega^{\prime}}\right), \forall \omega^{\prime} \in \mathscr{D}^{\prime}
$$

simulates a possible sequence of hourly loads $\tilde{l}_{\omega^{\prime}}$, if the same sequence of temperature forecasts of $\omega^{\prime} \in \mathscr{D}^{\prime}$ were to apply to $\mathscr{D}$. Once the simulated possible sequences of hourly load for each day in day type set $\mathscr{D}^{\prime}$ are obtained, a possible transformation between $\left\{\tilde{l}_{\omega^{\prime}}\right\}$ and $\left\{l_{\omega^{\prime}}\right\}$ can be found.

There are many possible approaches to transforming $l_{\omega^{\prime}}$ to $\tilde{l}_{\omega^{\prime}}$, but for easily transforming $\tilde{l}_{\omega^{\prime}}$ back to $l_{\omega^{\prime}}$ for the purpose of prediction in the original scale, the transform function is restricted to be linear:

$$
\tilde{l}_{\omega^{\prime}}=\Lambda l_{\omega^{\prime}}+\zeta_{\omega^{\prime}}, \forall \omega^{\prime} \in \mathscr{D}^{\prime}
$$

where $\Lambda$ is a matrix obtained by minimizing the error vectors $\left\{\zeta_{\omega^{\prime}}\right\}$ under a specified norm; i.e., $\|\cdot\|_{L_{1}}$. In addition, the transformation function is further designed to be a diagonal matrix to avoid singularity when computing $\Lambda^{-1}$. Although any day in a week can be viewed as a reference day type, we assign the reference days set $\mathscr{D}$ as all Wednesdays in a segment in a season to minimize the influences of weekends.

\subsection{Epi-spline based day-ahead hourly models}

Motivated by the power of epi-splines to derive term and volatility structures associated with financial markets (Wets and Bianchi, 2006), and its successful applications in numerous areas (Royset and Wets, 2014), in this paper epi-splines are applied to model hourly load on transformed reference day load data, corresponding weather forecast and time in each segment of a season for each zone. Section 3.3.1 introduces epi-splines. Sections 3.3.2 and 3.3.3 present two epi-spline based short-term load models. Unlike most short-term regression models for load, both of our models approximate the whole daily load curve at once to capture the serial correlations among hourly load values. 


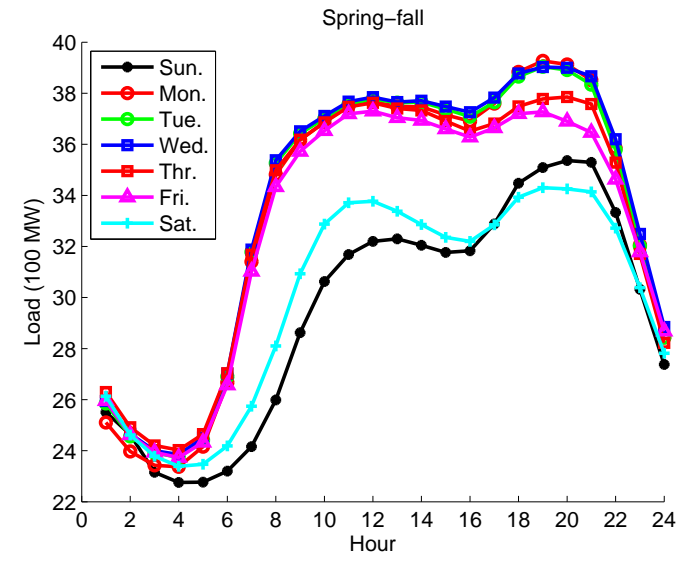

(a) Spring-fall

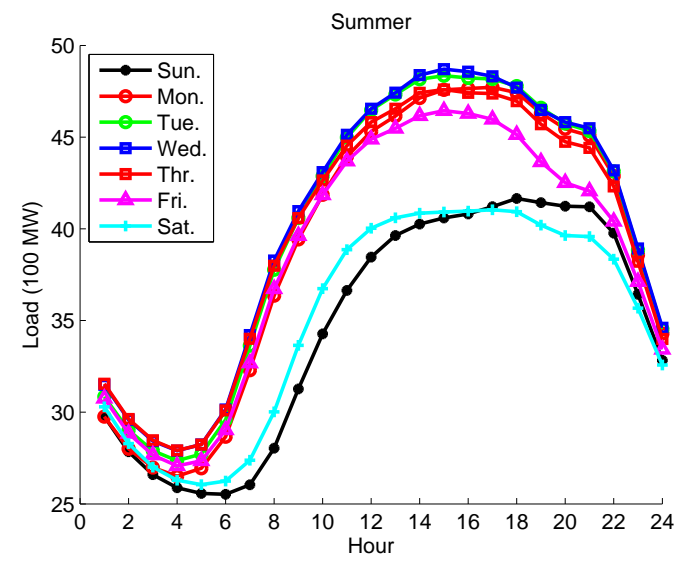

(b) Summer

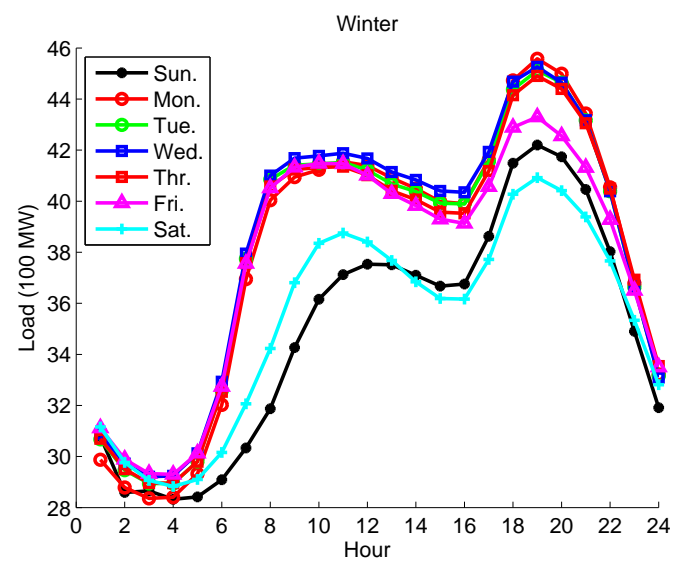

(c) Winter

Figure 3.3 Average daily load sequence in each day type in Apr. 2009 - Mar. 2011, CT 


\subsubsection{Approximation by epi-spline}

An epi-spline aims to approximate a twice-continuous differentiable function $f(r)$ over an interval $[0, \rho]$ (any interval $[\alpha, \beta]$ can be transformed to such interval). The whole interval $[0, \rho]$ is partitioned into $K$ equal subintervals $\mathscr{A}_{k}=\left(r_{k-1}, r_{k}\right]$ where $r_{0}=0$ and $r_{k}=r_{k-1}+\delta, k=1, \ldots, K$. By applying a piece-wise constant function

$$
a(r)=a_{k}, r \in \mathscr{A}_{k}
$$

to approximate the second derivative $f^{\prime \prime}(r)$ over each subinterval $\mathscr{A}_{k}$, the first derivative $f^{\prime}(r), r \in \mathscr{A}_{k}$ will be approximated by

$$
s^{\prime}(r)=w_{0}+\int_{0}^{r} a(\eta) d \eta=w_{0}+\delta \sum_{i=1}^{k-1} a_{i}+\left(r-r_{k-1}\right) a_{k} .
$$

Furthermore, the function $f(r), r \in \mathscr{A}_{k}$ is approximated by integrating $s^{\prime}(r)$ :

$$
\begin{aligned}
s(r) & =v_{0}+\int_{0}^{r} s^{\prime}(\eta) d \eta=v_{0}+\sum_{i=1}^{k-1} \int_{r_{i-1}}^{r_{i}} s^{\prime}(\eta) d \eta+\int_{r_{k-1}}^{r} s^{\prime}(\eta) d \eta \\
& =v_{0}+w_{0} r+\delta \sum_{i=1}^{k-1}\left(r-r_{i-1}+\frac{\delta}{2}\right) a_{i}+\frac{1}{2}\left(r-r_{k-1}\right)^{2} a_{k}
\end{aligned}
$$

where $v_{0}$ and $w_{0}$ are constants. In particular, if $r=r_{k}$, (3.6) can be simplified as:

$$
s\left(r_{k}\right)=v_{0}+r_{k} w_{0}+\delta^{2} \sum_{i=1}^{k}\left(k-i+\frac{1}{2}\right) a_{i} .
$$

Supposing the influence of weather condition; e.g., temperature, on load can be described as a continuous differentiable function of time, an epi-spline (3.6) can be used to model this effect.

\subsubsection{Weather conditional epi-spline (WEp) load model}

Figure 3.4 shows that in summer, the electricity consumption level increases as the forecast dew point temperature rises, even if the forecast temperature changes in a small range. Therefore, dew point temperature (humidity) is viewed as another factor in the short-term load model for summer.

Throughout this paper, $\mathscr{J}$ denotes the set of days, $\mathscr{H}=\{1,2, \cdots, 24\}$ the set of hours in a day, and

each hour in a day $j \in \mathscr{J}$ is divided into $\gamma$ equal subintervals, with $\gamma=1 / \delta$. Let $l_{j}^{h}$ be the actual load in hour $h \in \mathscr{H}$ in day $j \in \mathscr{J}$, and $t_{j}^{h}$ and $d_{j}^{h}$ be the forecast temperature and dew point temperature, respectively, for hour $h$ in day $j$ from the day ahead. Approximation functions $s_{t}(h)$ and $s_{d}(h)$ are 


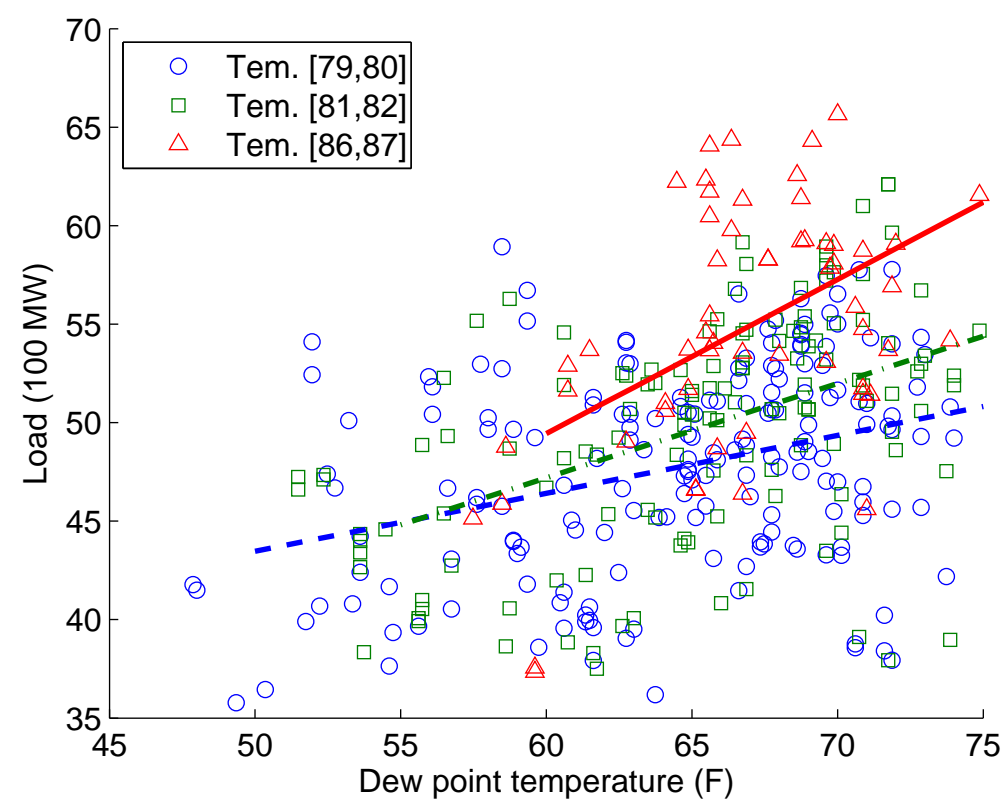

Figure 3.4 Load vs. dew point temperature forecast for hours within specified range of temperature forecast in summer, CT

found following (3.7) to model the impacts from temperature and dew point temperature, respectively, on electricity consumption for $h \in \mathscr{H}$. Because these weather variables may have different impacts on electricity consumption in a specified season, constants $\mu_{t}$ and $\mu_{d}$ are applied to weight such impacts. The short-term load model, WEp, is given as follows:

$$
l_{j}^{h}=\mu_{t} s_{t}(h) t_{j}^{h}+\mu_{d} s_{d}(h) d_{j}^{h}+e_{j}^{h}, \forall h \in \mathscr{H}, \forall j \in \mathscr{J}
$$

where $e_{j}^{h}$ represents the fitted model error in hour $h$ of day $j$. The related parameters of the load model are identified by minimizing $\left\|e_{j}^{h}\right\|_{1}$, in a linear program as follows: 


$$
\begin{aligned}
& \min _{\boldsymbol{v}_{\mathbf{0}}, \boldsymbol{w}_{\mathbf{0}}, \boldsymbol{a}, \boldsymbol{b}} \sum_{j \in \mathscr{J}} \sum_{h=1}^{24} \varepsilon_{j}^{h} \\
& \text { s.t. } \varepsilon_{j}^{h} \geq l_{j}^{h}-\mu_{t}\left(v_{0}^{t}+h w_{0}^{t}+\frac{1}{\gamma^{2}} \sum_{i=1}^{\gamma h}\left(\gamma h-i+\frac{1}{2}\right) a_{i}\right) t_{j}^{h} \\
& -\mu_{d}\left(v_{0}^{d}+h w_{0}^{d}+\frac{1}{\gamma^{2}} \sum_{i=1}^{\gamma h}\left(\gamma h-i+\frac{1}{2}\right) b_{i}\right) d_{j}^{h}, \forall j \in \mathscr{J}, h \in \mathscr{H} \\
& \varepsilon_{j}^{h} \geq-l_{j}^{h}+\mu_{t}\left(v_{0}^{t}+h w_{0}^{t}+\frac{1}{\gamma^{2}} \sum_{i=1}^{\gamma h}\left(\gamma h-i+\frac{1}{2}\right) a_{i}\right) t_{j}^{h} \\
& +\mu_{d}\left(v_{0}^{d}+h w_{0}^{d}+\frac{1}{\gamma^{2}} \sum_{i=1}^{\gamma h}\left(\gamma h-i+\frac{1}{2}\right) b_{i}\right) d_{j}^{h}, \forall j \in \mathscr{J}, h \in \mathscr{H} \\
& \varepsilon_{j}^{h} \geq 0, \forall j \in \mathscr{J}, h \in \mathscr{H}
\end{aligned}
$$

Clearly, $\varepsilon_{j}^{h}=\left|e_{j}^{h}\right|$ in (3.9) - (3.12). Once the model parameters $\boldsymbol{v}_{\mathbf{0}}, \boldsymbol{w}_{\mathbf{0}}, \boldsymbol{a}$ and $\boldsymbol{b}$ are obtained, a dayahead hourly load forecast for a specified day in the same segment is computed by applying formula (3.13) to the corresponding temperature and dew point temperature forecast.

$$
\hat{l}_{j}^{h}=\mu_{t} s_{t}(h) t_{j}^{h}+\mu_{d} s_{d}(h) d_{j}^{h}, \forall h \in \mathscr{H}, \forall j \in \mathscr{J}
$$

Parameters in the load model (3.8) can also be estimated by minimizing $\left\|e_{j}^{h}\right\|_{2}$ or $\left\|e_{j}^{h}\right\|_{\infty}$. The minimization of the fitted errors under $L_{2}$ norm results in a quadratic program, but minimizing $\left\|e_{j}^{h}\right\|_{\infty}$, the maximum fitted errors, over the training set leads to a linear program. The resulted linear program is modified by replacing $\varepsilon_{j}^{h}$ with single decision variable $\varepsilon$ in (3.10) - (3.12), and $\min _{\boldsymbol{v}_{\mathbf{0}}, \boldsymbol{w}_{\mathbf{0}}, \boldsymbol{a}, \boldsymbol{b}} \varepsilon$ in (3.9). This paper only presents load forecasts result from adopting $L_{1}$ norm, because it leads to better load forecast than the other two norms.

\subsubsection{Fundamental load incorporated weather conditional epi-spline (FWEp) load model}

Section 3.3.2 provides an epi-spline based hourly load model that depends only on weather conditions. However, electricity consumption is also strongly driven by human behavior in commercial, industrial and residential sectors over time. For example, higher load requirement tends to appear in the daytime and evening, while lower load requirements are seen in the late night and early morning in each season, as Fig. 3.5 shows. Moreover, peak load and peak temperature do not necessarily coincide. Therefore, an extra parameter may be needed to capture these non-weather dependent hourly load 
patterns; i.e., the average load for each hour in a given segment in each season, as Feng et al. (2014) proposes. However, the average load trajectory might not satisfactorily capture the non-weather dependent load pattern. In this paper we include this model-component in the error-minimizing procedure by using an additional epi-spline $s(h)$ that is a function of time only. For the convenience of terminology, we use fundamental load instead of non-weather dependent load pattern in the rest of the paper.
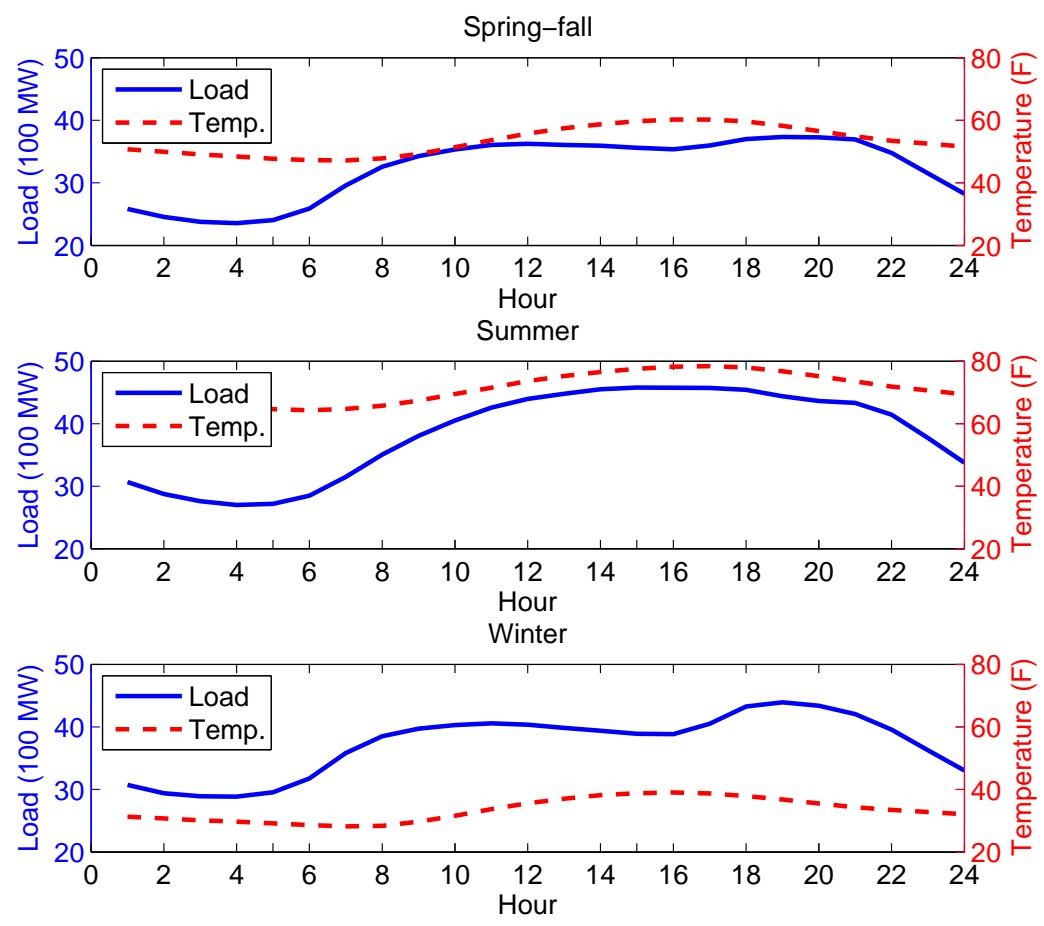

Figure 3.5 Average hourly temperature vs. average hourly load through a day in Apr. 2009 - Mar. 2011, CT

The FWEp model is given by:

$$
l_{j}^{h}=s(h)+\mu_{t} s_{t}(h) t_{j}^{h}+\mu_{d} s_{d}(h) d_{j}^{h}+e_{j}^{h}, \forall h \in \mathscr{H}, \forall j \in \mathscr{J}
$$

where $\mu_{t}$ and $\mu_{d}$ weight the impacts from temperature and dew point temperature on electricity consumption level, as before. Similar to model (3.9) - (3.12), model (3.15) - (3.17) is a linear program to 
estimate related parameters by minimizing the $L_{1}$ norm of errors, where $\varepsilon_{j}^{h}=\left|e_{j}^{h}\right|$.

$$
\begin{aligned}
\min _{v_{\mathbf{0}}, \boldsymbol{w}_{\mathbf{0}}, \boldsymbol{a}, \boldsymbol{b}, \boldsymbol{c}} \sum_{j \in \mathscr{J}} \sum_{h=1}^{24} \varepsilon_{j}^{h} \\
\text { s.t. } \varepsilon_{j}^{h} \geq l_{j}^{h}-\left(v_{0}^{h}+h w_{0}^{h}+\frac{1}{\gamma^{2}} \sum_{i=1}^{\gamma h}\left(\gamma h-i+\frac{1}{2}\right) a_{i}\right) \\
-\mu_{t}\left(v_{0}^{t}+h w_{0}^{t}+\frac{1}{\gamma^{2}} \sum_{i=1}^{\gamma h}\left(\gamma h-i+\frac{1}{2}\right) b_{i}\right) t_{j}^{h} \\
-\mu_{d}\left(v_{0}^{d}+h w_{0}^{d}+\frac{1}{\gamma^{2}} \sum_{i=1}^{\gamma h}\left(\gamma h-i+\frac{1}{2}\right) c_{i}\right) d_{j}^{h}, \forall j \in \mathscr{J}, h \in \mathscr{H} \\
\varepsilon_{j}^{h} \geq-l_{j}^{h}+\left(v_{0}^{h}+h w_{0}^{h}+\frac{1}{\gamma^{2}} \sum_{i=1}^{\gamma h}\left(\gamma h-i+\frac{1}{2}\right) a_{i}\right) \\
+\mu_{t}\left(v_{0}^{t}+h w_{0}^{t}+\frac{1}{\gamma^{2}} \sum_{i=1}^{\gamma h}\left(\gamma h-i+\frac{1}{2}\right) b_{i}\right) t_{j}^{h} \\
+\mu_{d}\left(v_{0}^{d}+h w_{0}^{d}+\frac{1}{\gamma^{2}} \sum_{i=1}^{\gamma h}\left(\gamma h-i+\frac{1}{2}\right) c_{i}\right) d_{j}^{h}, \forall j \in \mathscr{J}, h \in \mathscr{H} \\
\varepsilon_{j}^{h} \geq 0, \forall j \in \mathscr{J}, \forall h \in \mathscr{H}
\end{aligned}
$$

Similarly, if model parameters $\boldsymbol{v}_{\mathbf{0}}, \boldsymbol{w}_{\mathbf{0}}, \boldsymbol{a}, \boldsymbol{b}$ and $\boldsymbol{c}$ are obtained by optimizing (3.15) - (3.18), hourly load forecast for a day $j$ can be directly achieved given the corresponding temperature and dew point temperature forecast, as shown in formula (3.19).

$$
\hat{l}_{j}^{h}=s(h)+\mu_{t} s_{t}(h) t_{j}^{h}+\mu_{d} s_{d}(h) d_{j}^{h}, \forall h \in \mathscr{H}, \forall j \in \mathscr{J}
$$

Comparisons among WEp, FWEp, and the approach to estimating fundamental load using averaging load (AL) (Feng et al., 2014) are provided in Section 3.4.

\subsection{Case study}

Hourly load data and corresponding weather forecasts were collected from each zone in ISO-NE for the time period ranging from April 2009 to March 2012. As previous papers have discussed, public holidays must be differentiated from regular weekdays, because they have different electricity consumption patterns. In this paper, New Year's Day, Memorial Day, Independence Day, Labor Day, Thanksgiving and Christmas, are all considered as Sunday (Hong, 2011). Moreover, because of power system outages that resulted from extreme weather conditions, the day ranges $2010-2-5$ to $2010-2-7,2011-8-28$ to 
2011-8-31 and 2011-10-27 to 2011-11-4 were not considered in the short-term load model. The division of each season in Section 3.2.1 is further refined to create four seasons of a year, but a division point of a season may be in the middle of a month instead of at the beginning or at the end of a month because experimentation revealed that it would generally improve the prediction accuracy of FWEp.

The proposed two hourly load models, WEp and FWEp, were first fitted on a training set consisting of data from April 2009 to March 2011, and were then validated on the test set encompassing April 2011 to March 2012. The three treatments of fundamental load include ignoring it as in WEp, approximating it with an epi-spline as in FWEp, and substituting $s(h)$ in FWEp with the average load for each hour (Feng et al., 2014). All of these models are implemented in Python and Pyomo (Sandia National Laboratories, 2013a) on a Dell laptop with 6GB RAM. Table 3.2 summarizes the mean absolute percentage errors (MAPEs) of the predictions in the test set for each model. The MAPE is defined as:

$$
\text { MAPE }=\frac{\sum_{\mathscr{H}} \sum_{\mathscr{J}}\left|e_{j}^{h}\right|}{|\mathscr{H}| \times|\mathscr{J}|} .
$$

Table 3.1 Refined seasons

\begin{aligned} & \hline \hline Season name Range \\ & \hline Spring Apr.1 - May.14 \\ & Summer May.15 - Sep.14 \\ & Fall Sep.15 - Nov.30 \\ & Winter Jan.1 - Mar.31, Dec. \\ & \hline\end{aligned}

From Table 3.2, it is evident that more accurate hourly load predictions will result in the test set, Apr. 2011 - Mar. 2012, especially in seasons Spring, Fall and Winter, if extra parameters are used to estimate fundamental load. Table 3.2 also displays that estimating fundamental load in an optimization problem rather than using average load trajectory will improve accuracy further. Therefore, only performance of FWEp will be further investigated in the rest of the paper.

In practice, polynomial (Hagan and Behr, 1987; Moral-Carcedo and Vicens-Otero, 2005; Hong et al., 2011) is usually considered as one way to depict the nonlinear relationship between load and weather condition in production use. Therefore, two recent representative regression method based short-term load models (Hong, 2011; Charlton and Singleton, 2014) are considered as two benchmark models to show that FWEp can be competitive to capture the nonlinear relationship between temperature forecast and load, while attaining a narrower band of prediction errors. Unlike FWEp, either Hong 
Table 3.2 MAPEs of prediction errors of WEp, FWEp and AL in Apr. 2011 - Mar. 2012 (\%)

\begin{tabular}{lrrrrrrrrrrrr}
\hline \hline Zone & \multicolumn{3}{c}{ Spring } & \multicolumn{3}{c}{ Summer } & \multicolumn{3}{c}{ Fall } & \multicolumn{3}{c}{ Winter } \\
& WEp & FWEp & AL & WEp & FWEp & AL & WEp & FWEp & AL & WEp & FWEp & AL \\
\hline CT & 8.29 & 2.64 & 3.01 & 4.90 & 3.40 & 6.70 & 7.38 & 4.12 & 4.59 & 15.40 & 3.55 & 4.73 \\
ME & 10.23 & 5.02 & 4.34 & 4.93 & 3.81 & 4.95 & 9.93 & 3.92 & 3.94 & 19.57 & 3.19 & 3.80 \\
NH & 11.60 & 3.62 & 3.75 & 4.57 & 2.96 & 5.67 & 9.43 & 3.86 & 3.47 & 22.03 & 3.52 & 4.49 \\
NEMASSBOST & 8.70 & 2.55 & 2.84 & 4.10 & 2.86 & 5.96 & 6.85 & 3.43 & 3.55 & 15.63 & 3.28 & 4.02 \\
RI & 8.32 & 2.44 & 2.57 & 5.07 & 3.41 & 7.05 & 7.66 & 4.58 & 4.05 & 15.06 & 3.35 & 3.99 \\
SEMASS & 8.10 & 2.64 & 2.64 & 5.16 & 3.40 & 6.79 & 7.76 & 4.49 & 4.07 & 15.66 & 3.47 & 4.38 \\
VT & 11.38 & 2.44 & 2.48 & 3.89 & 2.44 & 3.86 & 10.77 & 2.85 & 2.69 & 28.02 & 4.21 & 5.16 \\
WCMASS & 9.60 & 2.61 & 2.75 & 4.11 & 3.03 & 5.82 & 8.21 & 3.54 & 4.00 & 18.79 & 3.36 & 4.47 \\
\hline
\end{tabular}

(2011) or (Charlton and Singleton, 2014) identifies a load model for each hour in a day type for each month or each season. The two load models are given in (3.21) and (3.22), and called the Hong and C\&S models, respectively.

\section{Hong model}

$$
\begin{aligned}
l_{j}^{h}= & \beta_{0}+\beta_{1} T+\beta_{2} D_{i(j)} H_{h}+\beta_{3} M_{k}+\beta_{4} M_{k} t_{j}^{h}+\beta_{5} M_{k} t_{j}^{h^{2}}+\beta_{6} M_{k} t_{j}^{h^{3}} \\
& +\beta_{7} H_{h} t_{j}^{h}+\beta_{8} H_{h} t_{j}^{h^{2}}+\beta_{9} H_{h} t_{j}^{h^{3}}+e_{j}^{h}, \forall j \in \mathscr{J}, h \in \mathscr{H}
\end{aligned}
$$

where $T$ is the load increasing trend, while $D_{i(j)}, i(j)=1, \cdots 7, H_{h}, h=1, \cdots, 24$ and $M_{k}, k=1, \cdots, 12$ are dummy variables, representing 7 day types in a week, 24 hours in a day, and 12 months in a year, respectively.

Charlton and Singleton (2014) divide a year into four seasons: spring, summer, fall and winter; and divide days into two types: weekday and weekend. For each hour of a day type in a season, the load model is given as follows:

\section{C\&S model}

$$
\begin{aligned}
l_{j}^{h}= & \alpha_{1}+\alpha_{2} \tau+\alpha_{3} t_{j}^{h}+\alpha_{4} t_{j}^{h} \tau+\alpha_{5} t_{j}^{h^{2}}+\alpha_{6} t_{j}^{h^{2}} \tau+\alpha_{7} \tau^{\prime}+\alpha_{8} t_{j}^{h} \tau^{\prime} \\
& +\alpha_{9} t_{j}^{h^{2}} \tau^{\prime}+e_{j}^{h}, \forall j \in \mathscr{J}, h \in \mathscr{H}
\end{aligned}
$$

where $\tau$ is the day number in the sequence of days, and $\tau^{\prime}$ the day number within the specified season. The above two benchmark models, (3.21) and (3.22), are both implemented by R. Charlton and Singleton (2014), employed several other approaches to improve performance of the short-term load model, 
including using forecasts from multiple weather stations instead of one, applying a local average technique, and smoothing the temperature forecast. To compare models on the same basis, we did not incorporate these techniques to forecast load ISO-NE in the test set. However, these useful techniques could be applied to improve accuracy of hourly load forecasting in future work.

Table 3.3 reports MAPEs for each zone in ISO-NE for all seasons in the test set. From Table 3.3, FWEp provides more accurate predictions in nearly all zones than the other two methods do in summer. In addition, FWEp can provide better or competitive predictions in spring and winter. In fall, FWEp seems to be slightly less accurate than the better of the two other methods.

Table 3.3 MAPEs of prediction errors of FWEp, Hong and C\&S in Apr. 2011 - Mar. 2012 (\%)

\begin{tabular}{|c|c|c|c|c|c|c|c|c|c|c|c|c|}
\hline \multirow[t]{2}{*}{ Zone } & \multicolumn{3}{|c|}{ Spring } & \multicolumn{3}{|c|}{ Summer } & \multicolumn{3}{|c|}{ Fall } & \multicolumn{3}{|c|}{ Winter } \\
\hline & FWEp & Hong & $\mathrm{C} \& \mathrm{~S}$ & FWEp & Hong & $C \& S$ & FWEp & Hong & $\mathrm{C} \& \mathrm{~S}$ & FWEp & Hong & $\mathrm{C} \& \mathrm{~S}$ \\
\hline CT & 2.64 & 3.07 & 3.30 & 3.40 & 4.26 & 4.97 & 4.12 & 3.31 & 3.24 & 3.55 & 3.06 & 3.53 \\
\hline ME & 5.02 & 3.35 & 4.29 & 3.81 & 5.38 & 3.65 & 3.92 & 5.36 & 3.44 & 3.19 & 3.38 & 3.08 \\
\hline $\mathrm{NH}$ & 3.62 & 3.29 & 4.56 & 2.96 & 3.41 & 4.25 & 3.86 & 2.72 & 2.92 & 3.52 & 2.66 & 3.56 \\
\hline NEMASSBOST & 2.55 & 2.48 & 2.88 & 2.86 & 4.06 & 4.38 & 3.43 & 3.95 & 2.98 & 3.28 & 3.90 & 3.18 \\
\hline RI & 2.44 & 2.62 & 3.10 & 3.41 & 4.29 & 5.27 & 4.58 & 3.70 & 3.11 & 3.35 & 2.92 & 3.05 \\
\hline SEMASS & 2.64 & 2.85 & 3.61 & 3.40 & 4.14 & 5.35 & 4.49 & 3.86 & 3.09 & 3.47 & 3.25 & 3.25 \\
\hline VT & 2.44 & 2.33 & 2.99 & 2.44 & 3.10 & 2.74 & 2.85 & 3.74 & 2.78 & 4.21 & 3.52 & 4.02 \\
\hline WCMASS & 2.61 & 2.93 & 3.47 & 3.03 & 3.61 & 4.01 & 3.54 & 4.00 & 3.31 & 3.36 & 4.04 & 3.35 \\
\hline
\end{tabular}

Root mean square errors (RMSE) for each zone of each short-term load model is summarized in Table 3.4. FWEp results in smaller RMSE in most zones, especially in CT, which accounts for approximately $25 \%$ of the load in ISO-NE. The RMSE is defined as:

$$
\mathrm{RMSE}=\sqrt{\frac{\sum_{j \in \mathscr{J}} \sum_{h \in \mathscr{H}}\left(e_{j}^{h}\right)^{2}}{|\mathscr{J}| \times|\mathscr{H}|-n}}
$$

where $n$ is the number of parameters of the model.

Table 3.4 RMSEs of prediction errors of FWEp and other two benchmark models in Apr. 2011 - Mar. 2012 (MW)

\begin{tabular}{lrrr}
\hline \hline Zone & FWEp & Hong & C\&S \\
\hline CT & 180.62 & 187.08 & 243.28 \\
ME & 61.77 & 68.69 & 67.03 \\
NH & 64.05 & 56.71 & 77.78 \\
NEMASSBOST & 133.54 & 155.86 & 184.05 \\
RI & 53.27 & 52.08 & 70.13 \\
SEMASS & 96.50 & 98.04 & 138.10 \\
VT & 28.49 & 27.92 & 32.06 \\
WCMASS & 93.50 & 102.51 & 118.28 \\
\hline
\end{tabular}

Because of the space limitation and the fact that CT has the highest load among ISO-NE zones, 
box plots of prediction errors for each method for all seasons in CT are given in Fig. 3.6 - Fig. 3.9. In Fig. 3.6, error bands from FWEp are narrower in hours 10 to 21, in which most residential, commercial and industrial electricity demands occur. Besides, the distributions of prediction errors from FWEp have less bias. In summer, as shown in Fig. 3.7, FWEp not only provides tighter error distributions in most hours, but also has less biased distributions of prediction errors for each hour. According to Fig. 3.8, wider distributions of prediction errors result from the C\&S model than from FWEp and Hong, and they are also highly biased. FWEp does not dominate the Hong model in load value prediction, but the boxplots of its prediction errors are similar. However, FWEp again provides less biased distributions of prediction errors, especially in hours 10 to 21. In winter, FWEp does not provide as accurate prediction as the other two methods do before 10 a.m., but it provides similarly accurate and less biased predictions between hours 10 and 21.

Fig. 3.10 summarizes the distributions of prediction errors in the test set for each model over ISONE. FWEp yields narrower distributions of prediction errors, and less biased error distribution compared to the benchmark models. The auto correlation function (ACF) is commonly used to compute serial correlations of a time series, but may be distorted in this application when lags span parts of consecutive days to which different weather forecasts apply. To restrict the attention only to the serial correlations among load values in the same day, a modified ACF, called intraday ACF (InACF), has been applied to measure the serial correlations. The sample InACF at lag $\kappa$ for load forecast $\left\{\hat{l}_{j}^{h}\right\}_{\mathscr{J}}^{\mathscr{H}}$ from a specified load model is calculated as:

$$
\hat{\rho}_{\kappa}=\frac{\sum_{j \in \mathscr{J}} \sum_{h=1}^{24-\kappa}\left(\hat{l}_{j}^{h}-\hat{\bar{l}}\right)\left(\hat{l}_{j}^{h+\kappa}-\hat{\bar{l}}\right)}{\sum_{j \in \mathscr{J}} \sum_{h=1}^{24}\left(\hat{l}_{j}^{h}-\hat{\bar{l}}\right)^{2}}, \kappa=1,2, \cdots, 23
$$

where $\hat{\bar{l}}$ is the average value of series $\left\{\hat{l}_{j}^{h}\right\}$.

The sample InACF, $\hat{\rho}_{\kappa}^{\prime}, \kappa=1,2, \cdots, 23$, is also calculated for actual hourly load in the test set for the comparison purposes, as displayed in Fig. 3.11. The InACF in Fig. 3.11 indicates that daily load trajectories from FWEp and the two benchmark models keep very similar serial correlations to those from the actual load in a day. The mean absolute errors are plotted by hour in Fig. 3.12 to check whether the forecast daily load trajectories are realistic. From Fig. 3.12, FWEp predictions are closer to the actual trajectory in spring, summer and fall, in hours 10 to 21. In winter, FWEp provides similar daily load trajectory to the one from C\&S. The daily load trajectory from FWEp is better than that 
from the Hong model in most of the hours between 10 and 21, but worse in hours 17 to 19 . With the observations that FWEp provides more accurate predictions, and less biased prediction intervals in most hours, it is expected that day-ahead hourly load scenarios created from FWEp will follow practical load shapes.

\subsection{Conclusion}

In this paper, two short-term load models, WEp and FWEp, are based on weather forecasts and function approximation. From the comparisons to the actual hourly load in Apr. 2011 - Mar. 2012, FWEp presents more accurate predictions in all seasons than those from WEp, because FWEp also considers fundamental load patterns that are not influenced by temperature or dew point temperature. Two recent representative regression short-term load models are selected to investigate distributions of prediction errors. Compared to the benchmarks, FWEp not only obtains less biased and narrower distributions of prediction errors, but also preserves similar serial correlations among forecasted hourly load to the actual ones. From the perspective accuracy in each hour and relationships among hours, FWEp is suitable for generating practical load scenarios for day-ahead stochastic unit commitment given temperature and dew point temperature forecasts. One possible way to generate load scenarios from load forecast is following (Rios et al., 2013). The authors applied another method to assess the fundamental load, and provided an approach to estimating forecast error distributions and then generating load scenarios.

This paper's scope is restricted to the influences of time, temperature and dew point temperature on load, but the model structure can be easily extended to investigate additional influences from wind speed and cloud cover on load if these data are available. If there are many contributing factors for load forecast, modeling their interaction effects might be useful to improve the accuracy of a load forecast. However, the current structure of epi-spline based load model does not support the approximations of these interaction effects. One area for future work is to develop multi-variable epi-splines to model the interaction effects of multiple contributing factors. 


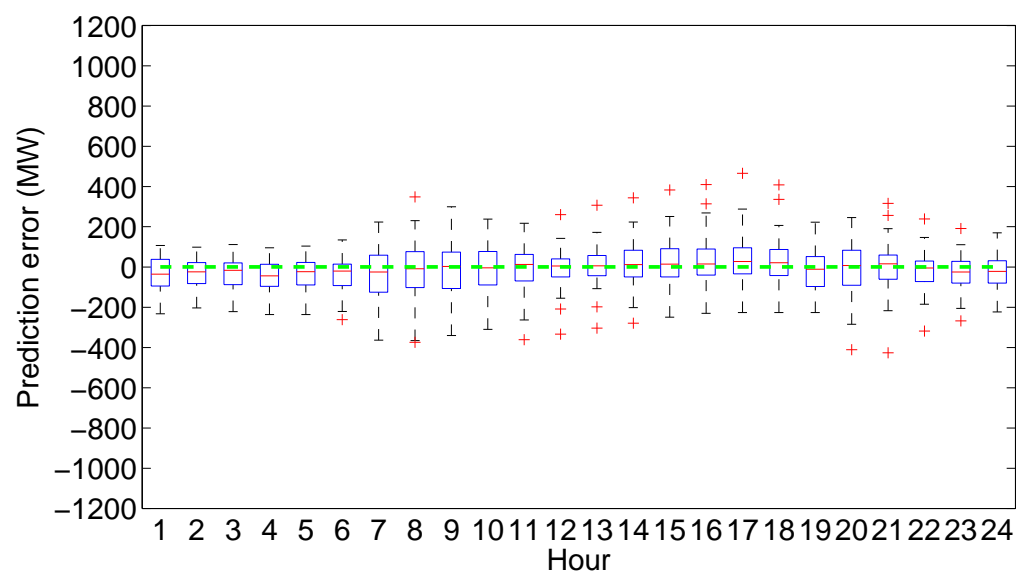

(a) FWEp: Zone CT

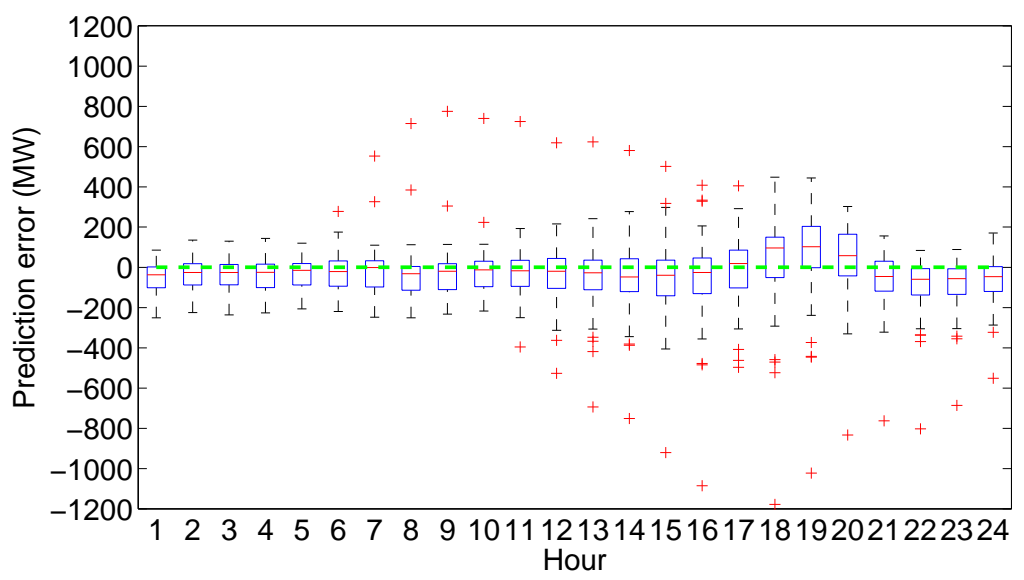

(b) Hong: Zone CT

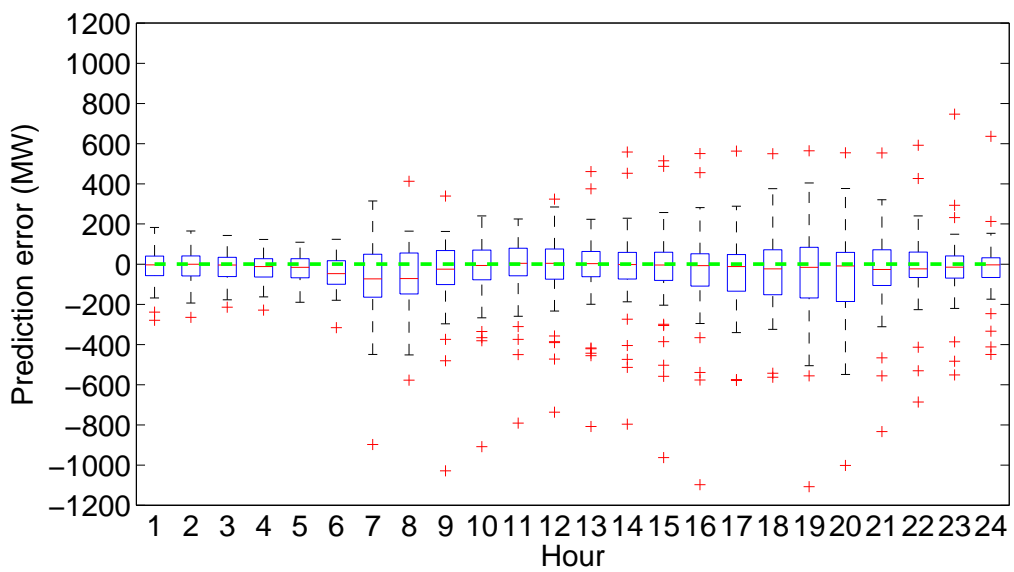

(c) C\&S: Zone CT

Figure 3.6 Box plots of prediction errors in Apr. 2011 - Mar. 2012 for FWEp and other two benchmark models: spring 


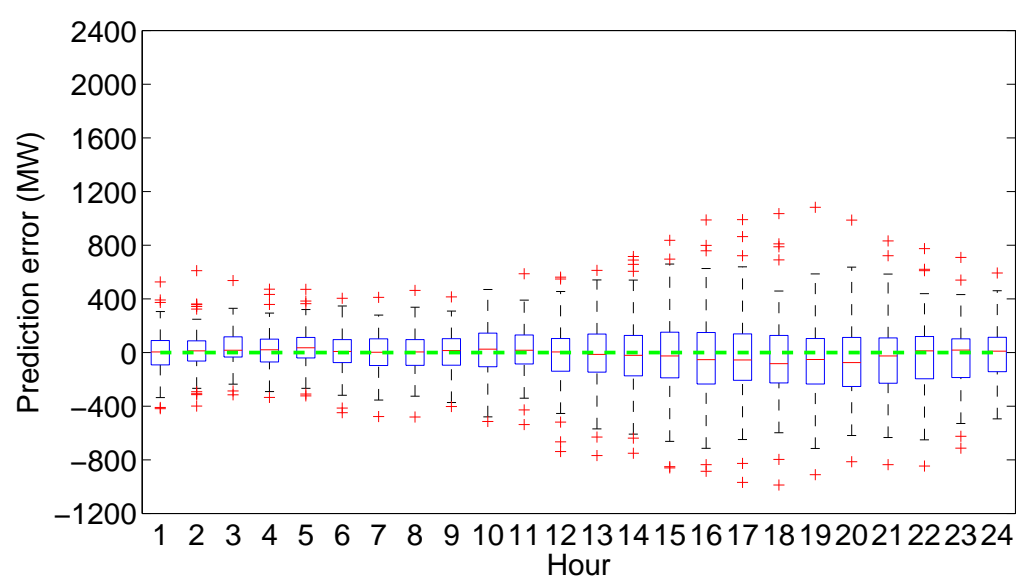

(a) FWEp: Zone CT

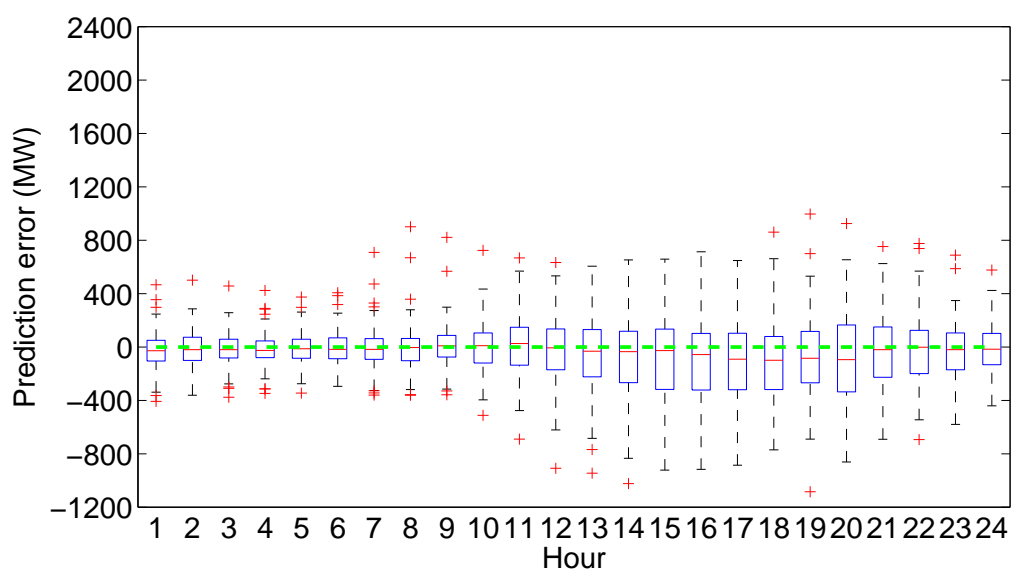

(b) Hong: Zone CT

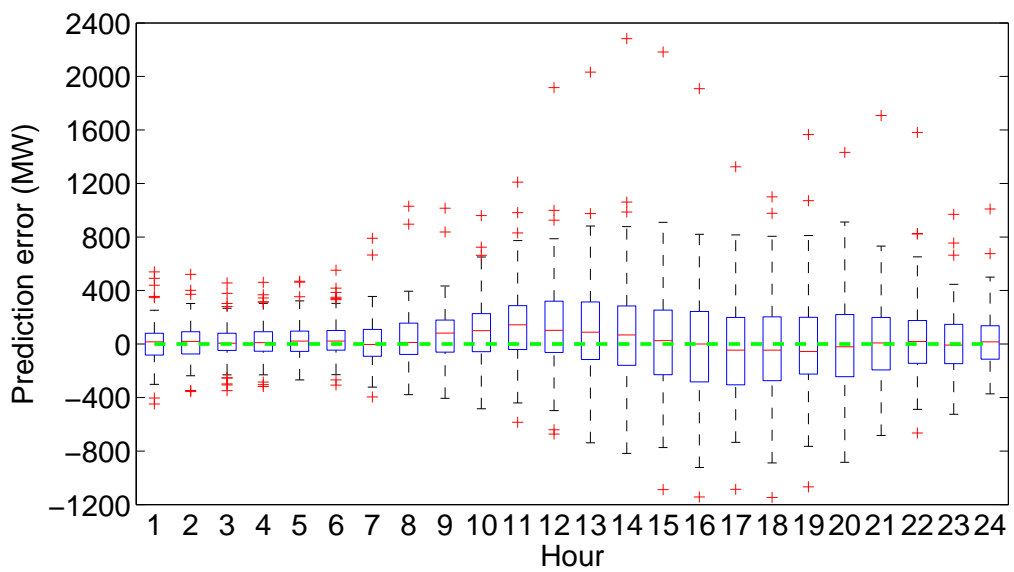

(c) C\&S: Zone CT

Figure 3.7 Box plots of prediction errors in Apr. 2011 - Mar. 2012 for FWEp and other two benchmark models: summer 


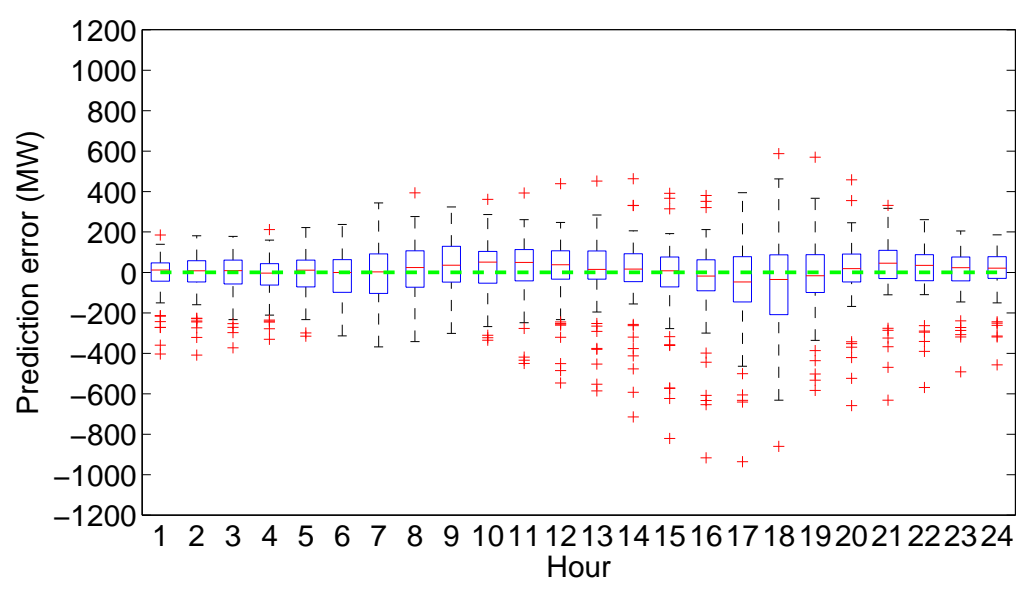

(a) FWEp: Zone CT

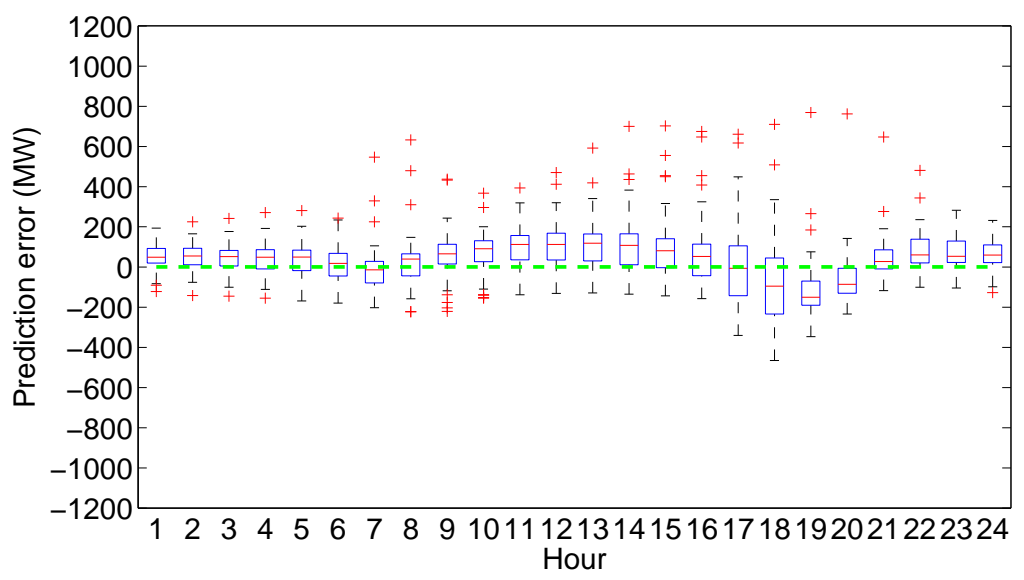

(b) Hong: Zone CT

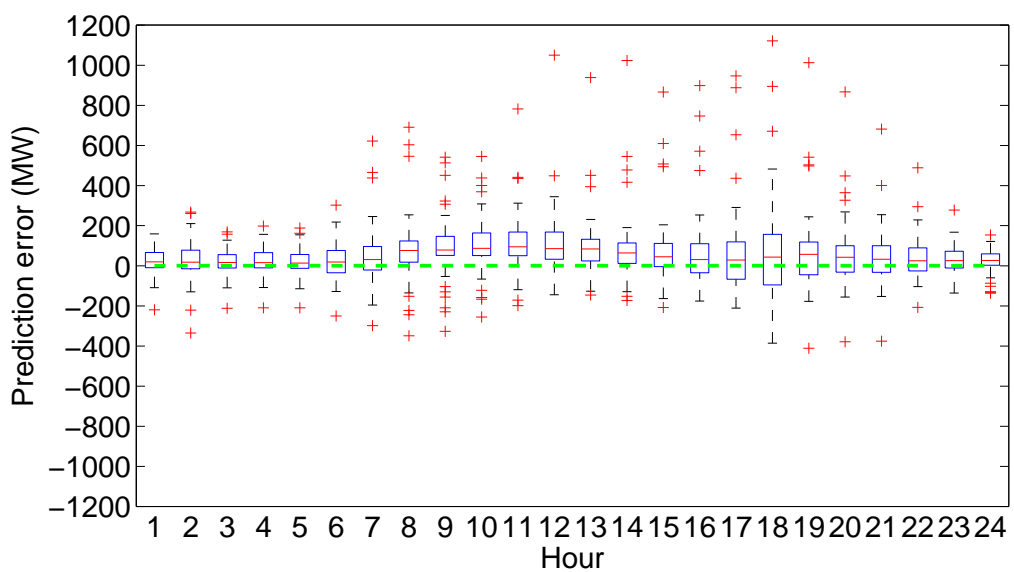

(c) C\&S: Zone CT

Figure 3.8 Box plots of prediction errors in Apr. 2011 - Mar. 2012 for FWEp and other two benchmark models: fall 


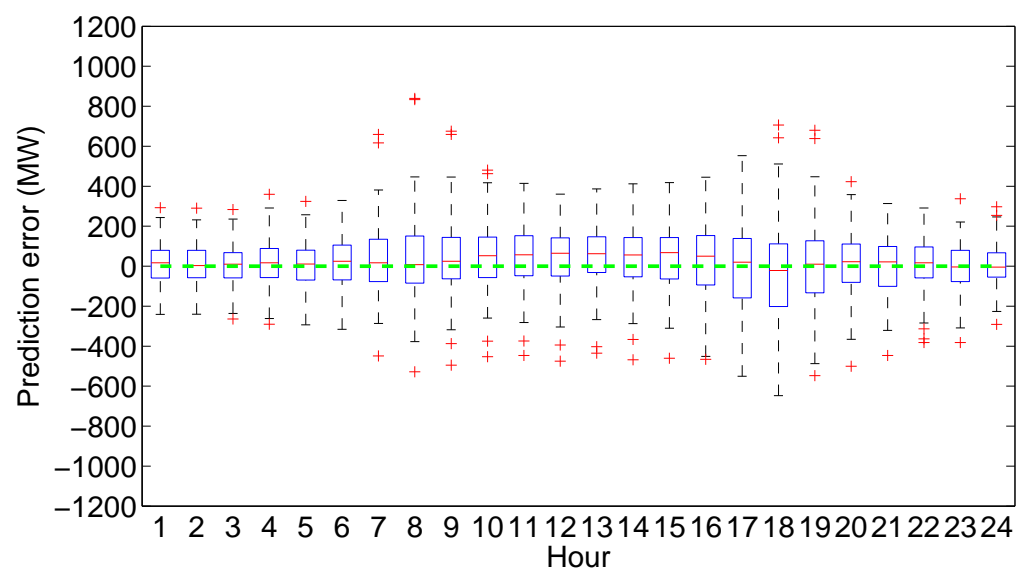

(a) FWEp: Zone CT

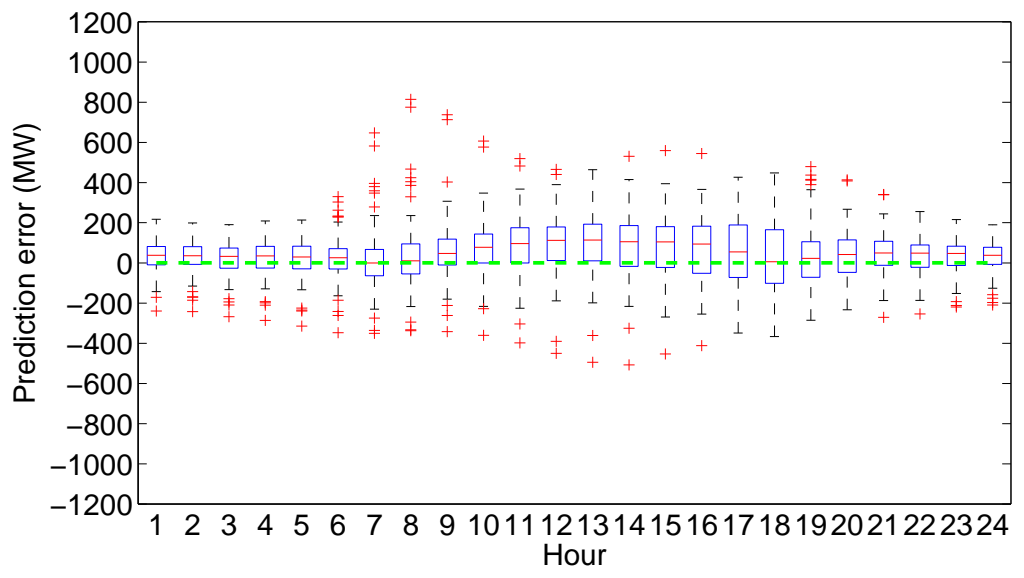

(b) Hong: Zone CT

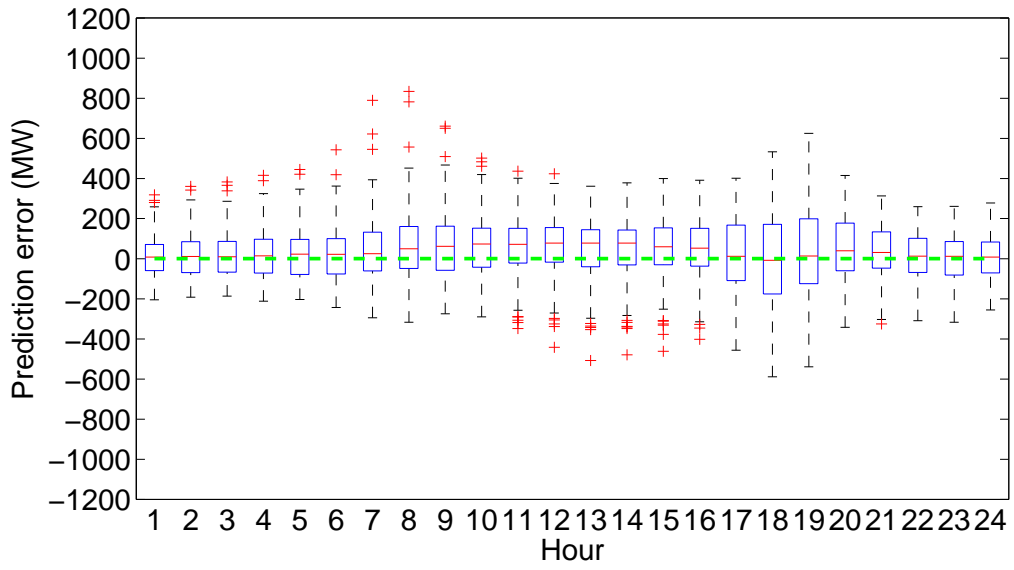

(c) C\&S: Zone CT

Figure 3.9 Box plots of prediction errors in Apr. 2011 - Mar. 2012 for FWEp and other two benchmark models: winter 


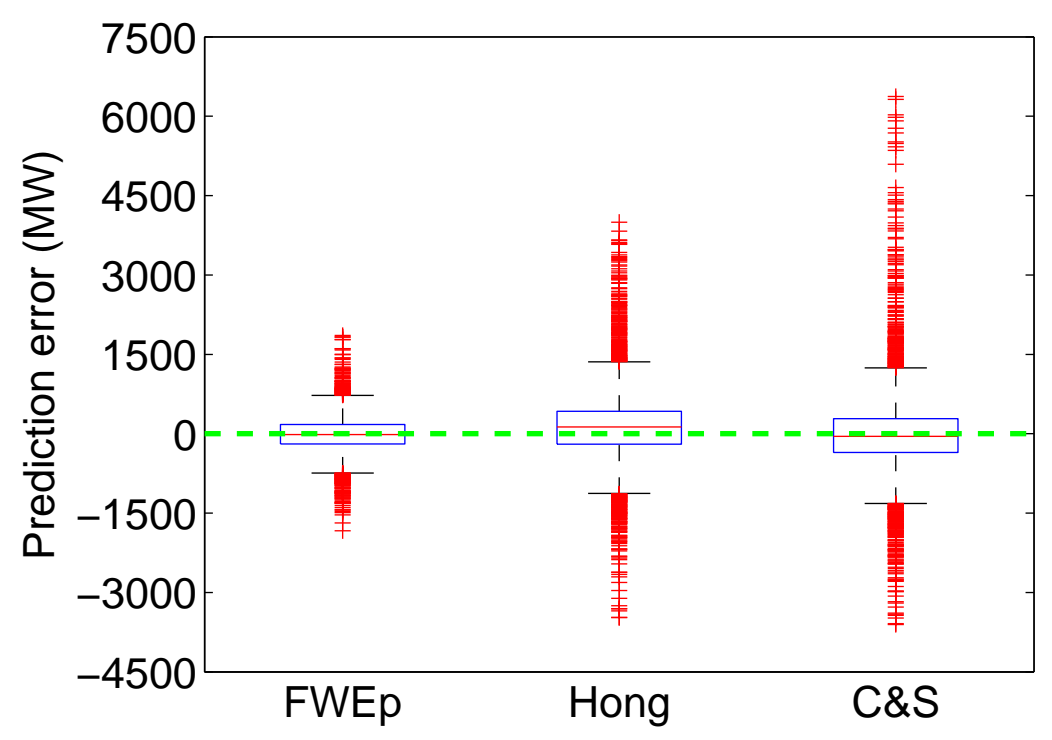

Figure 3.10 Box plot of prediction errors of FWEp and two benchmark models for ISO-NE in test set (Apr. 2011 - Mar. 2012)

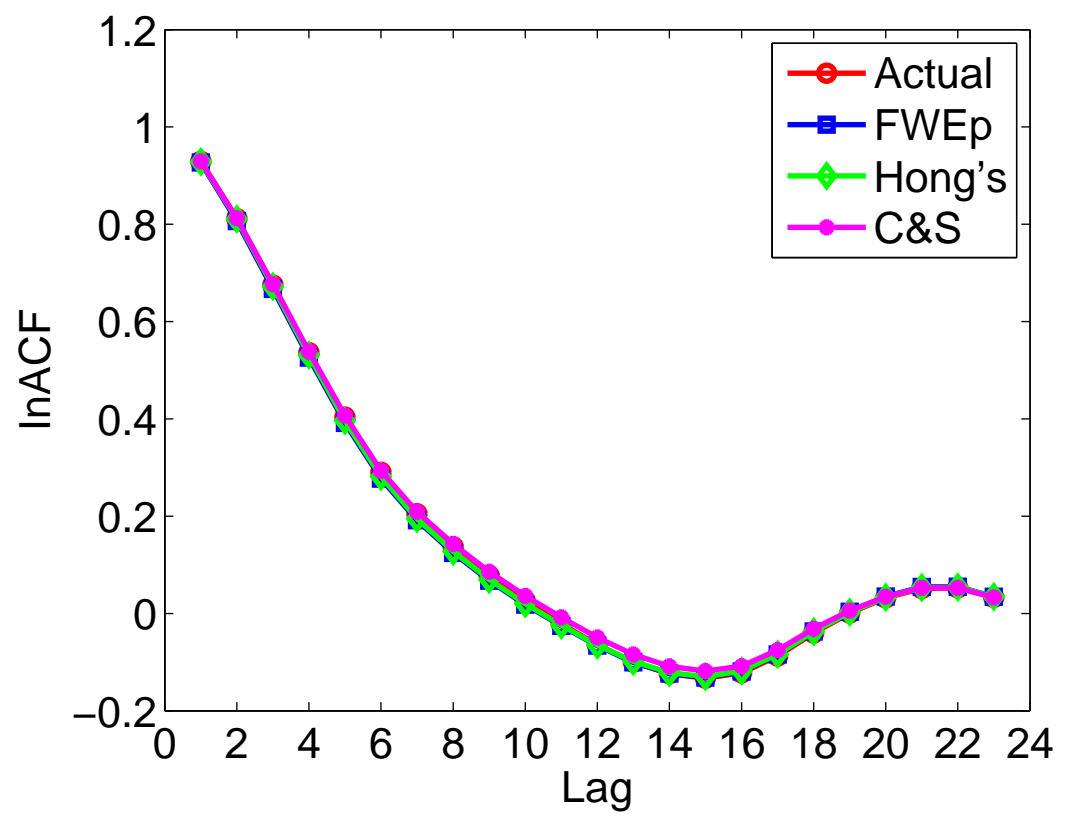

Figure 3.11 InACF of actual and forecast daily load trajectory of ISO-NE in test set (Apr. 2011 - Mar. 2012) 

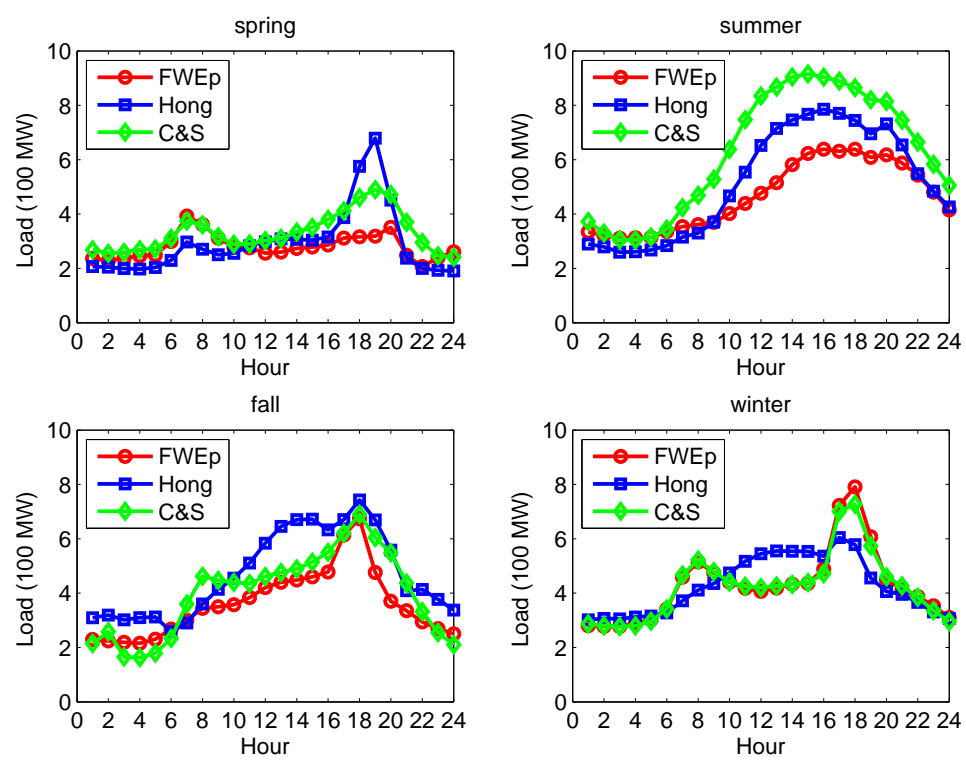

Figure 3.12 Average absolute errors in daily load trajectory of ISO-NE in test set (Apr. 2011 - Mar. 2012)

\subsection{Remark: Scenario generation}

The above sections, as parts of a journal paper draft, discuss a development of an appropriate dayahead short-term load model, the prerequisite of generating probabilistic load scenarios, for short-term day-ahead unit commitment. Because load scenario generation is a corporate work among Iowa State University, University of California at Davis and Sandia National Laboratories, we do not present every detail of scenario generation in this dissertation. For the detail, we refer to (Feng et al., 2014; Rios et al., 2013). This section only briefly introduces the mechanism of estimating density function of prediction errors, and the idea of generating load scenarios based on epi-spline model in this dessertation.

\subsubsection{Density estimation of prediction errors}

Similar to the idea shown in Section 3.3.1, it is also plausible to fit density of prediction errors using epi-spline. Instead of using the ordinary epi-spline, density function of prediction errors in hour $h, f(x)$, can be approximated by an exponential epi-spline for hour $h$ (Singham et al., 2013):

$$
\rho(x)=e^{-\varpi(x)}, x \in[\alpha, \beta]
$$


where $\varpi$ is the second-order one-dimension epi-spline. Suppose density function satisfies unimodal, then its curvature should be positive everywhere. By dividing the interval $[\alpha, \beta]$ into $N$ equal subintervals, and approximating the curvature by a piecewise constant function, epi-spline $\varpi(\cdot)$ will share the same formulation in (3.6).

To be consistent with the derivation process of (3.6), and implicitly assume errors will around the mean value before using maximum likelihood approach, the interval $[\alpha, \beta]$ is shifted to $[0, \beta-\alpha]$. Then an optimization problem is solved to estimate a finite number of parameters:

$$
\max \sum_{j \in \mathscr{J}} \ln e^{-\varpi\left(\tilde{e}^{j}\right)}
$$

s.t.

$$
\begin{aligned}
& \int_{0}^{\beta-\alpha} e^{-\varpi(\tau)} d \tau=1 \\
& \varpi\left(\tilde{e}^{j}\right)=\varpi_{0}+u_{0} \tilde{e}^{j}+\rho \sum_{l=1}^{k-1}\left(\tilde{e}^{j}-\tau_{l}+\frac{\rho}{2} a_{l}\right)+\frac{\left(\tilde{e}^{j}-\tau_{k-1}\right)^{2}}{2} a_{k} \\
& \varpi_{0} \geq 0, u_{0} \geq 0, a_{k} \in[0, \kappa], k=1, \cdots, N
\end{aligned}
$$

where set $\mathscr{J}$ represents days in the same segment, $\rho=\tau_{k}-\tau_{k-1}=(\beta-\alpha) / N ; \tau=0$ and $\tau_{k}=k \rho$ for $k=1, \cdots, N$. Problem (3.25) - (3.29) could be rewritten as the following problem:

$$
\begin{aligned}
& \quad \max \sum_{j \in \mathscr{J}} \ln e^{-\varpi\left(\tilde{e}_{j}\right)}+\int_{0}^{\beta-\alpha} e^{-\varpi(\tau)} d \tau \\
& \text { s.t. } \\
& \\
& \varpi\left(\tilde{e}^{j}\right)=\varpi_{0}+u_{0} \tilde{e}^{j}+\rho \sum_{l=1}^{k-1}\left(\tilde{e}^{j}-\tau_{l}+\frac{\rho}{2} a_{l}\right)+\frac{\left(\tilde{e}^{j}-\tau_{k-1}\right)^{2}}{2} a_{k} \\
& \varpi_{0} \geq 0, u_{0} \geq 0, a_{k} \in[0, \kappa], k=1, \cdots, N
\end{aligned}
$$

Once the probability density functions (PDF) for errors in each hour are obtained from problem (3.30) - (3.33), the cumulative distribution function (CDF) can be easily achieved. Instead of getting a closed form $\mathrm{CDF}$ for fitted errors for each hour in a day, a numerical CDF is obtained by cumulating probability to the interesting point after dividing the whole domain into a sequence of equal subintervals. To generate scenarios for day-ahead hourly load, we will first create a set of samples from the independent CDF of each hour we have estimated. 


\subsubsection{Probabilistic load scenario generation}

Once the prediction values, prediction errors and distribution of prediction errors are obtained, dayahead load scenarios could be generated following the procedure below (Feng et al., 2014).

\section{Scenario generation:}

1. Partition of day:

Suppose set $H=\left\{H_{i}\right\}, H_{1}=0, H_{|H|}=24, H_{i}<H_{i+1}$, includes all points dividing a day into several exclusive and exhaustive intervals, where $|H|$ represents the number of elements in set $H$. Fitting errors related to $H_{i}$ could be collected by applying

$$
\varepsilon_{d}^{H_{i}}=l_{d}^{H_{i}}-\psi_{H_{i}}(\omega, d, \mathscr{D})
$$

where function $\psi(\cdot)$ defines the epi-spline based function that approximates the relationship between load and weather condition, e.g (3.8), (3.14) or the epi-spline function built in ((Feng et al., 2014; Rios et al., 2013)). Thus, density function of prediction errors $\chi_{H_{i}}(\cdot)$, and corresponding distribution function $X_{H_{i}}(\cdot)$ could be estimated using the exponential epi-spline introduced in 3.6.1.

2. Division of prediction errors distribution function $X_{H_{i}}$ :

A sequence of points $\left\{c_{1}, c_{2}, \cdots, c_{K}\right\}$ divides interval $[0,1]$ into several exclusive and exhaustive subintervals. The resulted category $\mathscr{C}_{i}^{k}$ is defined by interval $\left[X_{H_{i}}^{-1}\left(c_{i}\right), X_{H_{i}}^{-1}\left(c_{i+1}\right)\right]$ for partition point $H_{i}$.

3. Classification of day $d$ :

For any day $d \in \mathscr{D}$, it will be classified to $\mathscr{D}_{i}^{k}$ if the following satisfies:

$$
d \in \mathscr{D}_{i}^{k} \leftrightarrow \varepsilon_{d}^{H_{i}} \in \mathscr{C}_{i}^{k}
$$

4. Combination of fitted values at limited hours:

A regression curve is fitted for each $\mathscr{D}_{i}^{k}$, and the fitted values $\hat{l}_{d}^{H_{i}}$ are calculated from $\psi\left(\omega, d, \mathscr{D}_{i}^{k}\right)$. Then, a convex combination is performed to avoid discontinuous at partition hours that define 
subinterval through a day, as below:

$$
\hat{l}_{d}^{h, k}=\frac{H_{i+1}-h}{H_{i+1}-H_{i}} \cdot \hat{l}_{d, i}^{h, k}+\frac{h-H_{i}}{H_{i+1}-H_{i}} \cdot \hat{l}_{d, i+1}^{h, k} .
$$

The fitted error for category $k$ in hour $h$ is calculated by

$$
\theta_{d}^{h, k}=l_{d}^{h}-\hat{l}_{d}^{h, k}
$$

to estimate corresponding density function $\chi_{h}(\cdot)$.

5. Division of error distribution function for category $k$ in hour $h$ :

The error distribution is divided several pieces by applying a set of points $\left\{m_{1}, m_{2}, \ldots, m_{N}\right\}$ to divide the interval $[0,1]$.

6. Calculation of conditional expectation:

The conditional expectation of each interval is calculated by following:

$$
\xi_{i, j}^{k}=\mathbb{E}\left[\theta_{j}^{k} \mid \theta_{j}^{k} \in\left[m_{z}, m_{z+1}\right]\right]=\frac{\int_{m_{z}}^{m_{z+1}} y \chi(y) d y}{\int_{m_{z}}^{m_{z+1}} \chi(y) d y} .
$$

These $\left\{\xi_{j, i}^{k}\right\}$ and $\hat{l}_{d}^{H_{i}, k}$ will lead to load scenarios. 


\subsection{Appendix A: Derivation of one-dimension epi-spline formulation}

In this part, the development of one-dimension epi-spline formulation (3.6) in Chapter 3 is pro-

vided in detail. Suppose the first derivative $f^{\prime}(r), r \in\left(r_{k-1}, r_{k}\right]$ is approximated by (3.5), then function value $f(r)$ is approximated by

$$
s(r)=v_{0}+\int_{0}^{r} s^{\prime}(\eta) d \eta=v_{0}+\sum_{i=1}^{k-1} \int_{r_{i-1}}^{r_{i}} s^{\prime}(\eta) d \eta+\int_{r_{k-1}}^{r} s^{\prime}(\eta) d \eta .
$$

We first calculate the third term of formulation (3.39), shown in (3.40):

$$
\begin{aligned}
\int_{r_{k-1}}^{r} s^{\prime}(\eta) d \eta & =\int_{r_{k-1}}^{r} w_{0}+\delta \sum_{i=1}^{k-1} a_{i}+\left(\eta-r_{k-1}\right) a_{k} d \eta \\
& =w_{0}\left(r-r_{k-1}\right)+\delta \sum_{i=1}^{k-1} a_{i}\left(r-r_{k-1}\right)+\frac{1}{2}\left(r-r_{k-1}\right)^{2} a_{k} .
\end{aligned}
$$

The second term of (3.39) follows:

$$
\begin{aligned}
\int_{r_{i-1}}^{r_{i}} s^{\prime}(\eta) d \eta & =\int_{r_{i-1}}^{r_{i}} w_{0}+\delta \sum_{j=1}^{i-1} a_{j}+\left(\eta-r_{i-1}\right) a_{i} d \eta \\
& =w_{0}\left(r_{i}-r_{i-1}\right)+\delta \sum_{j=1}^{i-1} a_{j}\left(r_{i}-r_{i-1}\right)+\frac{1}{2}\left(r_{i}-r_{i-1}\right)^{2} a_{i}
\end{aligned}
$$

The summation over $i=1, \cdots, k$ is made,

$$
\begin{aligned}
& \sum_{i=1}^{k-1} \int_{r_{i-1}}^{r_{i}} s^{\prime}(\eta) d \eta+\int_{r_{k-1}}^{r} s^{\prime}(\eta) d \eta \\
= & \sum_{i=1}^{k-1} w_{0}\left(r_{i}-r_{i-1}\right)+w_{0}\left(r-r_{k-1}\right)+\frac{1}{2}\left(r-r_{k-1}\right)^{2} a_{k}+\frac{1}{2} \sum_{i=1}^{k-1}\left(r_{i}-r_{i-1}\right)^{2} a_{i} \\
& +\delta \sum_{i=1}^{k-1} a_{i}\left(r-r_{k-1}\right)+\delta \sum_{i=2}^{k-1} \sum_{j=1}^{i-1} a_{j}\left(r_{i}-r_{i-1}\right) \\
= & w_{0} r+\frac{1}{2}\left(r-r_{k-1}\right)^{2} a_{k}+\frac{\delta^{2}}{2} \sum_{i=1}^{k-1} a_{i}+\delta \sum_{i=1}^{k-1} a_{i}\left(r-r_{i}\right)+\delta^{2} \sum_{i=2}^{k-1} \sum_{j=1}^{i-1} a_{j}
\end{aligned}
$$

the last term after the last " $=$ " derived from $\delta=r_{i}-r_{i-1}$.

Notice the following,

$$
\begin{aligned}
& a_{1}\left(r_{2}-r_{1}\right) \\
& +a_{1}\left(r_{3}-r_{2}\right) \quad+a_{2}\left(r_{3}-r_{2}\right) \\
& +a_{1}\left(r_{4}-r_{3}\right) \quad+a_{2}\left(r_{4}-r_{3}\right) \quad+a_{3}\left(r_{4}-r_{3}\right) \\
& \text { : } \quad \vdots \quad \ddots \\
& +a_{1}\left(r_{k-1}-r_{k-2}\right) \quad+a_{2}\left(r_{k-1}-r_{k-2}\right) \quad+a_{3}\left(r_{k-1}-r_{k-2}\right) \quad \cdots \quad+a_{k-2}\left(r_{k-1}-r_{k-2}\right)
\end{aligned}
$$


and according to (3.42), we could get the formulation (3.6)

$$
s(r)=v_{0}+w_{0} r+\delta \sum_{i=1}^{k-1}\left(r-r_{i}+\frac{\delta}{2}\right) a_{i}+\frac{1}{2}\left(r-r_{k-1}\right)^{2} a_{k} .
$$




\subsection{Appendix B: Some comments on high-dimension and multi-variable epi-spline construction}

This appendix provides a view of constructing epi-spline when multi-variables are considered. We first provide a way to construct 2-dimension epi-spline when two factors are considered in Section 3.8.1. An extension to multi-variables epi-spline is provided in this section, as well. The basis of constructing high-dimension and multi-variables epi-spline is discussed in Section 3.8.2.

\subsubsection{High-dimension and multi-variable epi-splines}

First, we suppose there are two factors which comprehensively influence the dependent variable, i.e. load value. Assuming there is a function $f(\cdot, \cdot)$ which could approximate such relationship, and $f \in \mathbb{C}^{2}$, then function $f$ could be written as:

$$
f(r, s)=\omega_{0}+v_{r} r+v_{s} s+\int_{r_{0}}^{r} \int_{\tau_{0}}^{\tau} a(\eta) d \eta d \tau+\int_{s_{0}}^{s} \int_{\varsigma_{0}}^{\varsigma} b(\zeta) d \zeta d \varsigma+\int_{r_{0}}^{r} \int_{s_{0}}^{s} c(\eta, \zeta) d \eta d \zeta
$$

where functions $a(\cdot), b(\cdot)$ and $c(\cdot)$ will be approximated by piece-wise constant functions along $r, s$ and $r \times s$ :

$$
\begin{gathered}
\hat{a}(r)=a_{i}, \forall r \in\left(r_{i-1}, r_{i}\right], \\
\hat{b}(s)=b_{j}, \forall s \in\left(s_{j-1}, s_{j}\right], \\
\hat{c}(r, s)=\alpha_{i j}, \forall(r, s) \in\left(r_{i-1}, r_{i}\right] \times\left(s_{j-1}, s_{j}\right] .
\end{gathered}
$$

Substituting (3.44)-(3.46) into (3.43), and replacing $\delta_{r}=r_{i}-r_{i-1}$ and $\delta_{s}=s_{j}-s_{j-1}$, we get a way to form two-dimension epi-spline:

$$
\begin{aligned}
\varpi(r, s) & =\omega_{0}+v_{r} r+v_{s} s+\frac{1}{2}\left(r-r_{k-1}\right)^{2} a_{k}+\frac{1}{2}\left(s-s_{l-1}\right)^{2} b_{l} \\
& +\delta_{r} \sum_{i=1}^{k-1}\left(r-r_{i}+\frac{\delta_{r}}{2}\right) a_{i}+\delta_{s} \sum_{j=1}^{l-1}\left(s-s_{j}+\frac{\delta_{s}}{2}\right) b_{j} \\
& +\delta_{r} \sum_{i=1}^{k-1} \alpha_{i l}\left(s-s_{l-1}\right)+\delta_{s} \sum_{j=1}^{l-1} \alpha_{k j}\left(r-r_{k-1}\right) \\
& +\delta_{r} \delta_{s} \sum_{i=1}^{k-1} \sum_{j=1}^{l-1} \alpha_{i j}+\alpha_{k l}\left(r-r_{k-1}\right)\left(s-s_{l-1}\right)
\end{aligned}
$$


From (3.47), load value $l$ in hour $h$ can be estimated as

$$
l_{h}=\psi(h)+\varpi(t, d)+e_{h}
$$

where $\psi(h)$ is the epi-spline from time, as discussed in Chapter 3 , and $\varpi(t, d)$ is the epi-spline related to weather variables, i.e. temperature and dew-point temperature.

According to (3.48), the load model can be obtained if the parameters are given by solving (3.49) - (3.51).

$$
\min \sum_{j \in \mathscr{J}} \sum_{h \in \mathscr{H}} e_{h}^{j}
$$

s.t.

$$
\begin{aligned}
& e_{h}^{j}>=l_{h}^{j}-\psi(h)-\varpi(t, d), \forall j \in \mathscr{J}, \forall h \in \mathscr{H} \\
& e_{h}^{j}>=\psi(h)+\varpi(t, d)-l_{h}^{j}, \forall j \in \mathscr{J}, \forall h \in \mathscr{H}
\end{aligned}
$$

Linear program (3.49) - (3.51) is employed to estimate parameters under $L_{1}$-norm, the formulations of other norms, i.e. $L_{2}$ and $L_{\infty}$, can also be achieved directly.

Some other possible information, i.e. first-order derivatives and control on peak-load hours, would be added to improve the models if they are necessary.

If one more factor needs to be considered in the load model, i.e. wind speed or cloud cover, a 3-dimension epi-spline seems to be necessary. Suppose $f \in \mathbb{C}^{2} \cap \mathscr{A}$ and $f: \mathbb{R}^{3} \mapsto \mathbb{R}$, where $\mathscr{A}$ is a set representing limitations on $f$, then function $f$ can be represented as

$$
\begin{aligned}
f(x, y, z) & =\omega_{0}+v_{x} x+v_{y} y+v_{z} z+\int_{x_{0}}^{x} \int_{\tau_{0}}^{\tau} x(s) d s d \tau+\int_{y_{0}}^{y} \int_{v_{0}}^{v} y(s) d s d v+\int_{z_{0}}^{z} \int_{\varsigma_{0}}^{\varsigma} z(s) d s d \varsigma \\
& +\int_{x_{0}}^{x} \int_{y_{0}}^{y} a(\tau, v) d \tau v+\int_{x_{0}}^{x} \int_{z_{0}}^{z} b(\tau, \varsigma) d \tau d \varsigma+\int_{y_{0}}^{y} \int_{z_{0}}^{z} c(v, \varsigma) d v d \varsigma
\end{aligned}
$$

Similar to the computation procedure of (3.43), we get the formulation of 3-dimension epi-spline 
(3.53).

$$
\begin{aligned}
\varpi(x, y, z) & =\omega_{0}+v_{x} x+v_{y} y+v_{z} z \\
& +\frac{a_{l}}{2}\left(x-x_{l-1}\right)^{2}+\frac{b_{m}}{2}\left(y-y_{m-1}\right)^{2}+\frac{c_{n}}{2}\left(z-z_{n-1}\right)^{2} \\
& +\delta_{x} \sum_{i=1}^{l-1}\left(x-x_{i}+\frac{\delta_{x}}{2}\right) a_{i}+\delta_{y} \sum_{j=1}^{m-1}\left(y-y_{j}+\frac{\delta_{y}}{2}\right) b_{j}+\delta_{z} \sum_{k=1}^{n-1}\left(z-z_{k}+\frac{\delta_{z}}{2}\right) c_{k} \\
& +\delta_{x} \sum_{i=1}^{l-1} \alpha_{i m}\left(y-y_{m-1}\right)+\delta_{y} \sum_{j=1}^{m-1} \alpha_{l j}\left(x-x_{l-1}\right)+\delta_{y} \sum_{j=1}^{m-1} \beta_{j n}\left(z-z_{n-1}\right) \\
& +\delta_{z} \sum_{k=1}^{n-1} \beta_{m k}\left(y-y_{m-1}\right)+\delta_{x} \sum_{i=1}^{l-1} \gamma_{i n}\left(z-z_{n-1}\right)+\delta_{z} \sum_{k=1}^{n-1} \gamma_{l k}\left(x-x_{l-1}\right) \\
& +\delta_{x} \delta_{y} \sum_{i=1}^{l-1} \sum_{j=1}^{m-1} \alpha_{i j}+\delta_{y} \delta_{z} \sum_{j=1}^{m-1} \sum_{k=1}^{n-1} \beta_{j k}+\delta_{x} \delta_{z} \sum_{i=1}^{l-1} \sum_{k=1}^{n-1} \gamma_{i k} \\
& +\alpha_{l m}\left(x-x_{l-1}\right)\left(y-y_{m-1}\right)+\beta_{m n}\left(y-y_{m-1}\right)\left(z-z_{n-1}\right)+\gamma_{l n}\left(x-x_{l-1}\right)\left(z-z_{n-1}\right)
\end{aligned}
$$

Analogously, the load model considering time $h$ and weather factors $t, d$ and $p$ can be written as:

$$
l_{h}=\psi(h)+\varpi(t, d, p)+e_{h} .
$$

The related parameters can be obtained by solving the following optimization problem (3.55) (3.57):

$$
\min \sum_{j \in \mathscr{J}} \sum_{h \in \mathscr{H}} e_{h}^{j}
$$

s.t.

$$
\begin{aligned}
& e_{h}^{j}>=l_{h}^{j}-\psi(h)-\varpi\left(t_{h}^{j}, d_{h}^{j}, p_{h}^{j}\right), \forall j \in \mathscr{J}, \forall h \in \mathscr{H} \\
& e_{h}^{j}>=\varpi\left(t_{h}^{j}, d_{h}^{j}, p_{h}^{j}\right)-l_{h}^{j}, \forall j \in \mathscr{J}, \forall h \in \mathscr{H} .
\end{aligned}
$$

\subsubsection{Theory of constructing high-dimension and multi-variables epi-splines}

The construction starts from the well known Second Fundamental Theorem of Calculus (Anton, 1999), which is the foundation of construction of one-dimension epi-spline. The Second Fundamental Theorem of Calculous is depicted as a lemma in this paper:

Lemma 1. Let $f$ and $F$ be real-valued function defined on $[a, b]$ such that the derivative of $F$ is $f$. That is, $F^{\prime}(x)=f(x), \forall x \in[a, b]$. If $f$ is Riemann integrable on $[a, b]$, then

$$
\int_{a}^{b} f(x) d x=F(b)-F(a) .
$$


The following part will discuss a way to establish high-dimension epi-spline and muti-variables epi-spline. Suppose a function $f(x, y)$ we are interested in can be separable into three exclusive parts $f_{1}(x), f_{2}(y)$ and $f_{3}(x, y)$. Here, the word "separable" indicates that the function $f$ can be written as a summation of several functions, i.e. $f(x, y)=f_{1}(x)+f_{2}(y)+f_{3}(x, y)$. In addition, we also require that the possible separable components of $f_{3}(x, y)$ can not be removed to either $f_{1}(x)$ or $f_{2}(y)$. Under the above requirements, we could claim that a function $f(x, y) \in C^{2}$ can be written as formulation (3.43) by the help from the following lemmas and theorems.

Lemma 2. Let function $f(x) \in C^{2}[a, b]$, then $f$ can be written as

$$
f(x)=v_{0}+w_{0} x+\int_{a}^{x} \int_{a}^{\tau} f^{\prime \prime}(\mu) d \mu d \tau .
$$

Proof. This lemma is just a straightforward consequence of Lemma 1.

Lemma 3. Let function $f(x, y)$ be twice differentiable over $[a, b] \times[c, d]$, and none of its component which could be separable can be written as $g(x)$ or $h(y)$, then $f(x, y)$ can be written as

$$
f(x, y)=v_{0}+\int_{c}^{x} \int_{d}^{y} r(\tau, \varsigma) d \tau d \varsigma
$$

where $r(x, y)=\frac{\partial^{2} f}{\partial x \partial y}$

Proof. It is also a straightforward result from any textbook on calculus. We do not prove it here.

Theorem 1. Let $f(x, y)$ be twice differentiable, and can be separated into three exclusive parts

$$
f(x, y)=f_{1}(x)+f_{2}(y)+f_{3}(x, y),
$$

where $f_{1}, f_{2}, f_{3} \in C^{2}$. In addition, none of components of $f_{3}$ which could be separated can be written as part of $f_{1}$ or $f_{2}$, then, $f(x, y)$ can be reformulated as

$$
f(x, y)=\omega_{0}+v_{x} x+v_{y} y+\int_{x_{0}}^{x} \int_{\tau_{0}}^{\tau} a(\eta) d \eta d \tau+\int_{y_{0}}^{y} \int_{\zeta_{0}}^{\zeta} b(\zeta) d \zeta d \varsigma+\int_{x_{0}}^{x} \int_{y_{0}}^{y} c(\eta, \zeta) d \eta d \zeta
$$

where

$$
a(x)=\frac{\partial^{2} f_{1}}{\partial x^{2}}
$$




$$
\begin{gathered}
b(y)=\frac{\partial^{2} f_{2}}{\partial y^{2}} \\
c(x, y)=\frac{\partial^{2} f_{3}}{\partial x \partial y} .
\end{gathered}
$$

Proof. Since $f_{1}$ and $f_{2}$ are both twice differentiable, according to Lemma 2, they can be written as

$$
\begin{aligned}
& f_{1}(x)=v_{x}+w_{x} x+\int_{x_{0}}^{x} \int_{x_{0}}^{\tau} a(\mu) d \mu d \tau \\
& f_{2}(x)=v_{y}+w_{y} y+\int_{y_{0}}^{y} \int_{y_{0}}^{\varsigma} b(\zeta) d \varsigma d \zeta .
\end{aligned}
$$

Moreover, because $f_{3}$ cannot be separated into parts which could be removed to $f_{1}$ or $f_{2}$, according to Lemma 3, it can be written as

$$
f_{3}(x, y)=v_{0}+\int_{x_{0}}^{x} \int_{y_{0}}^{y} c(\tau, \varsigma) d \tau d \varsigma
$$

After adding them together, we get the conclusion.

According to the above theorem, it is easy to get an extension form if $n$-dimension function is discussed. The related conclusion is summarized as the following corollary.

Corollary 1. Let $f: \mathbb{R}^{n} \mapsto \mathbb{R}$, and be twice differentiable. In addition, $f$ can be separated into several parts:

$$
f=\sum_{i=1}^{n} f_{i}+\sum_{j=1}^{n} \sum_{k>j}^{n} g_{i j}
$$

where, $f_{i}$ defined on $\mathbb{R}$ is a function of $x_{i}, g_{j k}\left(x_{j}, x_{k}\right)$ defined on $\mathbb{R}^{2}$, and $f_{i}, g_{j k} \in C^{2}$. Moreover, $g_{j k}$ is a function from which any split component can not be removed to $f_{i}, i=1, \cdots, n$. Then, function $f$ can be reformulated as

$$
\begin{aligned}
f\left(x_{1}, x_{2}, \cdots, x_{n}\right) & =\omega_{0}+\sum_{i=1}^{n} v_{i} x_{i}+\sum_{i=1}^{n} \int_{x_{i_{0}}}^{x_{i}} \int_{\eta_{i_{0}}}^{\eta_{i}} p\left(v_{i}\right) d v_{i} d \eta_{i} \\
& +\sum_{j=1}^{n} \sum_{k>j}^{n} \int_{x_{k_{0}}}^{x_{k}} \int_{x_{j_{0}}}^{x_{j}} q(\tau, v) d \tau d v
\end{aligned}
$$

The proof of Corollary 1 is intuitive by following the procedure of Theorem 1, we are not going to prove it. 


\title{
CHAPTER 4. SOLUTION SENSITIVITY-BASED SCENARIO REDUCTION FOR STOCHASTIC UNIT COMMITMENT
}

\author{
A paper published in Computational Management Science
}

Yonghan Feng and Sarah M. Ryan

\begin{abstract}
A two-stage stochastic program is formulated for day-ahead commitment of thermal generating units to minimize total expected cost considering uncertainties in the day-ahead load and the availability of variable generation resources. Commitments of thermal units in the stochastic reliability unit commitment are viewed as first-stage decisions, and dispatch is relegated to the second stage. It is challenging to solve such a stochastic program if many scenarios are incorporated. A heuristic scenario reduction method termed forward selection in recourse clusters (FSRC), which selects scenarios based on their cost and reliability impacts, is presented to alleviate the computational burden. In instances down-sampled from data for an Independent System Operator in the U.S., FSRC results in more reliable commitment schedules having similar costs, compared to those from a scenario reduction method based on probability metrics. Moreover, in a rolling horizon study, FSRC preserves solution quality even if the reduction is substantial.
\end{abstract}

\subsection{Introduction}

Unit commitment (UC) is a short-term scheduling problem in electric power systems. The goal is to identify on/off decisions for thermal generating units over a planning horizon to satisfy forecast demand, while minimizing the total startup, shutdown and generation costs under restrictions on unit operation and transmission over power lines. Physical constraints and wholesale electricity market rules require 
some of these scheduling decisions to be made on the day before they go into effect. As increasing amounts of renewable energy resources, such as wind and solar power, have been integrated into power systems, the uncertainty associated with conditions on the day ahead have increased - on the demand side due to load forecasting errors, and also on the supply side because of the intermittence of renewable energy and the difficulty of predicting its availability several hours in advance.

Stochastic programming is a promising approach to solving a UC problem under uncertainty from the perspective of minimizing total expected cost and/or some risk measures. In general, commitments are decided in the first stage before the real time information is realized, while decisions on generation amounts in each period, called economic dispatch (ED), can be delayed until the second stage after realizing actual information, such as load and variable resource availability. Compared to the deterministic UC, stochastic programming can achieve significant cost savings when uncertain resources are involved (Takriti et al., 1996; Carpentier et al., 1996). A crucial aspect of modeling to achieve a good solution in a stochastic program is to formulate a finite number of reasonable scenarios, or trajectories of the uncertain parameters over the scheduling horizon, from a stochastic process. Various methods have been developed for generating scenarios. A large number of paths can provide thorough coverage of the joint distributions but also severely limit the computational tractability of solving the stochastic program. To attain a reasonable amount of computation time, it is natural to attempt to approximate the original large set of scenarios with a smaller subset. Many researchers have contributed to the field of scenario reduction. A general approach based on stability analysis and probability metrics is represented by (Dupačová et al., 2003; Heitsch and Römisch, 2003), and has been widely used in power system studies. However, in recent studies of unit commitment with high levels of variable energy penetration, some doubts have been expressed about the practical utility of this approach (Botterud et al., 2011; Papavasiliou and Oren, 2013; Papavasiliou et al., 2011).

This paper develops a heuristic scenario reduction method for use with a two-stage stochastic program for unit commitment by following the decision maker's two major concerns: reliability and cost. We conjecture that better scenarios, as defined below, could be selected by considering their impacts on the first-stage decisions. The developed heuristic scenario reduction method, called forward selection in recourse clusters (FSRC), clusters scenarios according to their impact on solutions (measured by a solution sensitivity index), and then applies the well-known forward selection heuristic, fast forward 
selection (FFS) (Heitsch and Römisch, 2003), to select one representative scenario from each cluster. Similarity measurement is one of the application-specific aspects of FSRC; others aim to improve computational efficiency and clustering effectiveness. The FSRC method is investigated in the context of day-ahead stochastic reliability unit commitment (SRUC) with uncertain load and variable generation resources. In restructured wholesale electricity markets, reliability unit commitment problems are solved in the afternoon of the day before the target day, after the day-ahead market (based on demand bids and supply offers) has cleared, based on the system operator's forecast of conditions on the target day. In our case studies, we consider the variable generation resource, wind energy, to be nondispatchable - that is, we subtract the amount of available wind energy from the demand to yield net load to be satisfied by the thermal generators. But the scenario reduction heuristic can be adapted easily for models in which variable generation resources are dispatchable.

The contributions of this paper include the development and customization of a heuristic scenario reduction method that not only considers probabilities and distances among scenarios, but also follows the decision maker's concerns for reliability and economy. In case studies we find that more reliable commitment of units, from the perspective of less shortage and lower scheduling cost, can be obtained by optimizing against a subset of scenarios selected by FSRC rather than by FFS. In addition, numerical results for a rolling horizon SRUC show that FSRC keeps the solution time manageable while maintaining solution quality even when the reduction of the scenario set is substantial.

The rest of the paper is organized as follows. Section 4.2 reviews related literature on stochastic UC, in particular with high penetration of wind energy, and scenario reduction. Section 4.3 formulates a compact two-stage stochastic program for SRUC, the concrete counterpart of which is given in Appendix 4.8. Section 4.4 introduces a generic procedure of FSRC, its customization strategies and an approach for evaluating the sets of selected scenarios. Section 4.5 reports numerical results of investigations of applying FSRC to independent daily SRUC and rolling horizon SRUC over selected days based on data collected from an Independent System Operator in the U.S.. Section 4.6 concludes this paper. Finally, Section 4.7 remarks some extensions of FSRC. 


\subsection{Literature review}

Motivated by the uncertainties associated with variable generation as its penetration in power systems has increased, much attention recently has been devoted to applying stochastic programming in unit commitment. For instance, Bouffard et al. (2005) formulated a two-stage stochastic program for security-constrained unit commitment to address market-clearing, and Bouffard and Galiana (2008) analyzed the impact of wind energy penetration on reserve requirements in a small-scale model. Morales et al. (2012) used stochastic programming to co-optimize energy and reserve in an electricity pool with significant wind penetration. Tuohy et al. (2009) investigated the benefits of using stochastic UC to account for high penetration of wind energy. Ruiz et al. (2009) explicitly modeled uncertain resources and investigated the effect of reserve requirement in $\mathrm{UC}$ with penetration of wind energy from the perspective of an Independent System Operator, but the computational tests used only a small number of scenarios. Papavasiliou and co-authors used an alternative formulation obtained by distinguishing the commitment of slow-start and fast-start generators as first- and second-stage decisions, respectively (Papavasiliou and Oren, 2013; Papavasiliou et al., 2011). The economic effects of forecast accuracy on wind and uncertainty bounds have been analyzed by integrating a numerical weather prediction model into stochastic UC/ED (Constantinescu et al., 2011). Römisch and Vigerske (2010) summarized several techniques in stochastic programming applied to UC. While many of these studies employed stochastic UC models to investigate broader issues, such as the economic and reliability effects of incorporating large amounts of variable generation, our work is aimed at implementing stochastic unit commitment for daily use by system operators in their resource adequacy assessments.

In this context, the limited time available for computing a unit commitment schedule necessitates parsimony in the scenario set. The computation time strongly depends on the number of scenarios even if a decomposition method, such as Benders decomposition (Zheng et al., 2013; Liu et al., 2014), Lagrangian relaxation (Carøe and Schultz, 1998; Nowak and Römisch, 2000; Takriti et al., 2000), or a progressive hedging algorithm (Takriti et al., 1996) is applied. The size of the Benders master problem will increase dramatically if many scenarios are included. Progressive hedging requires some heuristic strategies to improve convergence if integer decision variables appear in the first-stage (Watson and Woodruff, 2011). Slow convergence in a Lagrangian relaxation algorithm may occur as the number 
of scenarios dramatically increases. Therefore, reducing the number of scenarios while closely approximating the stochastic processes of uncertain parameters becomes an attractive way to alleviate the computational burden.

An intuitive way to reduce the number of scenarios is to cluster them in specified periods according to their parameters, and represent scenarios in the same cluster by their expected values (Dupačová et al., 2000; Philpott et al., 2000; Latorre et al., 2007; Küchler and Vigerske, 2007). Sampling a subset of scenarios is another common approach (Kleywegt et al., 2002). Scenarios may be deleted based on an expected value of perfect information (EVPI) criterion (Dempster and Thompson, 1999). An importance sampling approach attempts to select scenarios according to their probability of occurrence and impact on operating cost (Papavasiliou and Oren, 2013). Other deletion rules include purely heuristic or ad hoc rules (Beltratti et al., 1999; Bertocchi et al., 2000) and ones that preserve the first and second order moments of the original scenarios (Carino et al., 1998).

A well-known line of research has developed scenario reduction methods to achieve stability in the objective function with respect to the scenarios used. The forward selection (FS) and backward reduction (BR) heuristics were developed to identify a subset of scenarios with minimal distance from the original set according to a mass transportation metric (Dupačová et al., 2003). Heitsch and Römisch (2003) proposed variants of these methods, including fast forward selection (FFS), which is more efficient in selection than FS and yields a reduced set more similar in distribution to the original set than BR does when the reduction is substantial. While these scenario reduction methods were derived according to the upper bound of Fortet-Mourier metrics instead of the metrics themselves, a refinement for twostage stochastic programs rigorously based on Fortet-Mourier metrics was also derived (Heitsch and Römisch, 2007). These methods were further extended to chance constrained and mixed-integer twostage stochastic programs, where they are stated with respect to cell discrepancy (or the Kolmogorov metric) (Henrion et al., 2009) or a certain polyhedral discrepancy (Henrion et al., 2008). Further extensions to multi-stage stochastic programs have been made (Heitsch and Römisch, 2011, 2009b; Heitsch et al., 2006; Heitsch and Römisch, 2009a). A nested distance between multistage scenario trees was introduced in (Pflug and Pichler, 2012) and algorithms for optimal quantization according to the nested distance were developed in (Timonina, 2013). Because of the encouraging numerical results reported in the initial work (Dupačová et al., 2003; Heitsch and Römisch, 2003), these methods have been ap- 
plied widely in power systems studies (Growe-Kuska et al., 2003; Morales et al., 2009; Wu et al., 2007; Tuohy et al., 2009; Wang et al., 2008; Fleten and Wallace, 2009).

Although the stability-based scenario reduction methods mentioned above have sound theoretical background, practical concerns have been raised concerning their use in two-stage stochastic unit commitment for large-scale integration of renewable energy. The modeler cannot explicitly identify scenarios that may impose significant influences on the performance of the unit commitment schedules, and scenarios selected by these methods may not be consistent with the moments of wind power time series (Papavasiliou et al., 2011; Papavasiliou and Oren, 2013). In addition, in a recent study FFS seemed not to dominate random sampling when reducing sets of scenarios to represent significant wind energy penetration (Botterud et al., 2011). To select scenarios that reflect the decision maker's concern in SRUC, it is plausible to apply a heuristic scenario reduction method designed for a two-stage stochastic generation expansion planning (Feng and Ryan, 2013). The heuristic method incorporates the impact of scenarios on first-stage decisions, as well as forward selection based on probability metrics. However, directly employing the same scenario reduction approach in SRUC would be computationally prohibitive because it measures scenario impacts by solving a mixed integer program for each scenario in the original set. To achieve tractable computation in the scenario reduction procedure itself, this paper proposes a related heuristic scenario reduction method, FSRC, which improves the computational efficiency of identifying scenarios with similar impacts on decisions. Moreover, FSRC also tracks the decision maker's concern for reliability in SRUC. Details of the FSRC method are provided in Section 4.4.

\subsection{Two-stage stochastic reliability unit commitment model}

The SRUC problem aims to identify a UC schedule that minimizes startup and shutdown costs as well as expected generation cost and penalties on load and reserve imbalances while satisfying operational restrictions over all scenarios. In this section, a compact two-stage SRUC model is given in (4.1) - (4.5), and its concrete counterpart, which extends the deterministic model in (Carrión and Arroyo, 2006), is provided in the Section 4.8. It is a two-stage stochastic program with relatively complete recourse provided by including slack variables in the energy balance and reserve requirement constraints. 
Scenarios represent different possible time series for load and renewable generation over the scheduling horizon. Uncertainties not explicitly modeled by the scenarios, such as generator and transmission line contingencies, are managed by including operating reserve requirements. The abstract form of the two-stage model is:

$$
\begin{gathered}
f(\mathscr{S})=\min _{x} c^{\top} x+\mathscr{Q}(x, \mathscr{S}) \\
\text { s.t. } \quad A x=b \\
x \text { binary }
\end{gathered}
$$

where

$$
\begin{aligned}
\mathscr{Q}(x, \mathscr{S}) & =E_{\mathscr{S}}[Q(x, s)] \\
Q(x, s) & =\min _{y_{s}}\left\{q_{s}^{\top} y_{s} \mid W y_{s}=h_{s}-T_{s} x\right\}
\end{aligned}
$$

The objective function (4.1) includes two parts: $c^{\top} x$, the costs related to commitment, $x$, of units; and $\mathscr{Q}(x, \mathscr{S})$, the expected value over a given set of scenarios in the second stage including optimal generation cost and penalties on load and reserve requirement imbalances given unit commitments in the first-stage, as shown in (4.4). The optimal commitment vector depends on $\mathscr{S}$, as we explore numerically in the case studies. Formula (4.2) describes the feasible region of $x$, following minimum up- and down-time constraints. Formula (4.5) minimizes generation cost and penalties on load and reserve requirement imbalances, $q_{s}^{\top} y_{s}$, after realizing each scenario given the commitment of units. Energy balance, transmission, and ramp rate constraints as well as generation level limitations, etc., related to every concrete scenario are also summarized in the feasible region described by (4.5). The proposed scenario reduction method is devised for model (4.1) - (4.5) but its steps must be customized for the concrete form described partially below in (4.6) - (4.11). Definitions of the following notation and a complete description of the constraints are included in Section 4.8.

Objective function The objective function of the concrete model is:

$$
\min \sum_{t \in \mathscr{T}} \sum_{g \in \mathscr{G}} c_{g t}^{u}\left(v_{g t}\right)+\sum_{s \in \mathscr{S}} \xi_{s} \zeta_{s}
$$


The first term of (4.6) is a piecewise linear cost function corresponding to $c^{\top} x$ in (4.1) and represents the total startup, shutdown, and no-load costs of committed units, represented by the binary unit status decision variables, $v_{g t}$, over all periods. The second term is the counterpart of $\mathscr{Q}(x)$, where $\xi_{s}$ is the scenario probability and $\zeta_{s}$ is the objective value upon realization of a specific scenario $s$ in the second stage. This consists of a piecewise linear generation cost, $c_{g t s}^{p}$, computed from the continuous generation levels $p_{g t s}$ for each unit $g$ in each period $t$, as well as penalties on imbalances in load satisfaction and reserve requirements, as described in (4.7):

$$
\zeta_{s}=\sum_{t \in \mathscr{T}} \sum_{g \in \mathscr{G}} c_{g t s}^{p}\left(p_{g t s}\right)+\sum_{t \in \mathscr{T}} \sum_{b \in \mathscr{B}}\left(\Gamma_{\alpha}^{+} \alpha_{b t s}^{+}+\Gamma_{\alpha}^{-} \alpha_{b t s}^{-}\right)+\sum_{t \in \mathscr{T}}\left(\Gamma_{\beta}^{+} \beta_{t s}^{+}+\Gamma_{\beta}^{-} \beta_{t s}^{-}\right) .
$$

Here, $\Gamma_{\alpha}^{+}$and $\Gamma_{\alpha}^{-}$are penalties set on shortage and excess in supply; i.e., $\alpha_{b t s}^{+}$and $\alpha_{b t s}^{-}$, respectively. Similarly, $\Gamma_{\beta}^{+}$and $\Gamma_{\beta}^{-}$are penalties on shortage and excess in required reserve, $\beta_{t s}^{+}$and $\beta_{t s}^{-}$.

Note that (4.7) requires as much demand for energy to be satisfied as possible. Energy balance and reserve requirement constraints are described in (4.8)-(4.9) and (4.10)-(4.11), respectively.

\section{Energy balance at each bus:}

$$
\begin{aligned}
\sum_{g \in \mathscr{G}(b)} p_{g t s} & +\sum_{\ell \in \mathscr{L}_{I}(b)} \omega_{\ell t s}-\sum_{\ell \in \mathscr{L}_{O}(b)} \omega_{\ell t s} \\
& +\alpha_{b t s}^{+}-\alpha_{b t s}^{-}=d_{b t s}, \forall b \in \mathscr{B}, \forall t \in \mathscr{T}, \forall s \in \mathscr{S} \\
& \alpha_{b t s}^{+}, \alpha_{b t s}^{-} \geq 0, \forall b \in \mathscr{B}, \forall t \in \mathscr{T}, \forall s \in \mathscr{S}
\end{aligned}
$$

The time unit of one hour is chosen so that generated power and energy share the same numerical value in each period for each unit. Formula (4.8) states that for each bus $b$, a shortage $\alpha_{b t s}^{+}$will result if the sum of energy amounts provided by each unit at that bus, $p_{g t s}$, and net energy transmitted to that bus on each line, $\omega_{\ell t s}$, is less than load $d_{b t s}$ in period $t$ in scenario $s$; or excess, $\alpha_{b t s}^{-}$, will occur if the sum is greater than the load. In this paper, the scenario-specific parameters $d_{b t s}$ represent net load computed by subtracting nondispatchable variable energy generation from load. With the increasing penetration of distributed variable generation, such as residential solar panels, uncertainty in the net load served by utilities and system operators will continue to increase. However, many operators are now able to dispatch wind and utility-scale solar plants. To model dispatchable variable generation, right-hand-sides of those generation limit constraints would also vary by scenario, as described in the Appendix. 


\section{Reserve requirements:}

$$
\begin{gathered}
\sum_{g \in \mathscr{G}} \bar{p}_{g t s}+\beta_{t s}^{+}-\beta_{t s}^{-}=\sum_{b \in \mathscr{B}} d_{b t s}+R_{t}, \forall t \in \mathscr{T}, \forall s \in \mathscr{S} \\
\beta_{t s}^{+}, \beta_{t s}^{-} \geq 0, \forall t \in \mathscr{T}, \forall s \in \mathscr{S}
\end{gathered}
$$

In formula (4.10), the reserve requirement, $R_{t}$, in each hour of the scheduling horizon requires that some spare capacity be available if needed to maintain reliability in case of contingencies that are not modeled in scenarios; e.g., outages of generators or transmission lines. The difference between the

maximum available generation level $\bar{p}_{g t s}$ and actual generation level $p_{g t s}$ represents the contribution of unit $g$ to meeting the reserve requirement in period $t$ in scenario $s$. Similar to (4.8), slack variables, $\beta_{t s}^{+}$ and $\beta_{t s}^{-}$, are introduced in (4.10), representing possible shortage and excess in the reserve requirement.

A motivation of employing stochastic programming in unit commitment is to determine an economical quantity of implicit reserves rather than explicitly requiring a fixed amount of reserve capacity to be available in case of errors in forecasting net load. In the absence of storage capabilities, physics requires that total generation equal total net load, so strictly speaking, "shortage" would mean shedding some load and "excess" would mean curtailing some generation. Practically, small positive values of the slack and surplus variables might simply result in tolerable short-term violations of capacity constraints or negotiated temporary reductions in consumption. Very risk-averse operators might prefer to include some fixed reserve constraints in the stochastic program to avoid either consequence (Ruiz et al., 2009). Besides providing relatively complete recourse, we use the slack and surplus variables to evaluate, against the whole set of scenarios, the quality of the unit commitment schedules obtained by solving with a subset of scenarios, as described in Section 4.4.3.

Details of other operational constraints, including transmission constraints, ramp rate constraints, etc., are described in Appendix 4.8.

\subsection{Scenario reduction}

A large number of scenarios may be generated to represent stochastic processes for the multiple uncertain parameters in a stochastic program. To reduce the computational effort for solving the stochastic mixed integer program, it is natural to explore methods to approximate a large number of 
generated scenarios with a modest-sized subset of scenarios, while keeping their main features. Our scenario reduction heuristic is developed in this section.

\subsubsection{Forward selection in recourse clusters (FSRC)}

Widely used methods for scenario reduction are based on probability metrics. Among these, the fast forward selection (FFS) method is often applied to select a subset of scenarios $\mathscr{S}^{\prime}$ from the original set $\mathscr{S}$, because numerical results indicate that the forward selection (FS) heuristic yields a more similar reduced distribution than the alternative, backward reduction, and FFS provides significant speedup over the original FS heuristic (Heitsch and Römisch, 2003). The distance between a subset $\mathscr{S}^{\prime}$ of the prescribed size and the remaining scenarios $\mathscr{S} \backslash \mathscr{S}^{\prime}$ can be computed by solving a mass transportation problem. Because identifying an optimal reduced set is a hard combinatorial problem, the FFS heuristic was developed as a tractable way to select one scenario at a time.

Although forward selection is based on stability analysis and, thus, indirectly considers the optimization objective, it accounts directly for only the scenario parameters and corresponding probabilities $\left\{\xi_{s}\right\}$. It does not directly consider the possible influences of scenarios on the decision variables or their costs. We conjecture that better performance could be achieved by considering these impacts in the selection process. Therefore, we propose a heuristic scenario reduction method, FSRC, which not only considers distances between selected scenarios and the deleted scenarios, but also directly measures influences from scenarios on decisions and costs.

Before introducing the FSRC algorithm, we discuss how to measure scenario impacts on decisions. A solution sensitivity index is created from decision variables or part of the objective function which could quantify differences among scenarios. For instance, in a UC problem, a scenario subproblem could be solved to identify the optimal hourly on/off status of each unit through the whole scheduling horizon assuming perfect information; thus, these decision variables could be considered as a solution sensitivity index. However, if there are hundreds of generators in the scheduling problem, considering the whole commitment decision vector would be unwieldy, and may allow features of scenarios to be blurred because of the inherent difficulties in high dimensional data analysis. Instead, total cost could serve to distinguish among scenarios because higher demand often results in higher generation cost. We denote the function that maps the first-stage decision, $x$, and the second-stage decision, $y_{s}$, to the $i$ th 
solution sensitivity index as $\mathscr{F}_{i}\left(x, y_{s}\right), i=1, \ldots, m$. Useful indices are problem-specific. Further discussion on forming solution sensitivity indices for stochastic unit commitment will be presented in section 4.4.2.2. To reduce the computational burden while maintaining solution quality, the selected subset of scenarios should retain some decision-relevant features of the whole set of scenarios. Therefore, it is intuitive to assign scenarios with similar characteristics, as measured by solution sensitivity indices, into the same group and select a representative scenario from each group. Our scenario reduction method follows this idea.

For a large mixed-integer program, solving the subproblem for each scenario may be too computationally intensive in itself. Instead, we find a feasible first-stage decision vector and then solve a dispatch linear program for each scenario. The corresponding optimal second stage decisions and costs are employed to reveal characteristics of scenarios.

Suppose the prescribed cardinality of selected scenario set $\mathscr{S}^{\prime}$ is $n$. For a two-stage stochastic program (4.1) - (4.5) with relatively complete recourse, a generic FSRC method is given in Algorithm 1.

\section{Algorithm 1. Forward Selection in Recourse Clusters (FSRC):}

1. Evaluate: For each $s \in \mathscr{S}$, identify an optimal $y_{s}^{*}$, given a feasible solution $\hat{x}$ of $A x=b$, by solving

$$
Q(\hat{x}, s)=\min _{y_{s}}\left\{q_{s}^{\top} y_{s} \mid W y_{s}=h_{s}-T_{s} \hat{x}\right\}
$$

2. Summarize: Compute solution sensitivity indices

$$
\mathscr{N}^{s}=\left[\mathscr{F}_{1}\left(\hat{x}, y_{s}^{*}\right), \mathscr{F}_{2}\left(\hat{x}, y_{s}^{*}\right), \cdots, \mathscr{F}_{m}\left(\hat{x}, y_{s}^{*}\right)\right]
$$

for $s \in \mathscr{S}$.

3. Cluster: Scale $\mathscr{F}_{i}, i=1, \cdots, m$, into similar magnitudes, denoted as $\hat{\mathscr{F}}_{i}, i=1, \cdots, m$. Assign weight $\omega_{i}$ to each $\hat{\mathscr{F}}_{i}, i=1, \cdots, m$, and compile them as

$$
V^{s}=\left[\omega_{1} \hat{\mathscr{F}}_{1}\left(\hat{x}, y_{s}^{*}\right), \omega_{2} \hat{\mathscr{F}}_{2}\left(\hat{x}, y_{s}^{*}\right), \cdots, \omega_{m} \hat{\mathscr{F}}_{m}\left(\hat{x}, y_{s}^{*}\right)\right]
$$

Form $n$ clusters on $\left\{V^{s}\right\}$ by the k-means method using an appropriate norm, and create the corresponding $n$ clusters in $\mathscr{S}$; 
4. Select: Use FFS to select one scenario from each cluster of the original scenarios.

Because characterization of scenario impact on decision variables is often problem-dependent, it is necessary to customize FSRC for different applications. The customization specifies how to identify solution sensitivity indices, and then create clusters accordingly. Discussion on customization strategies of FSRC for the SRUC problem follows.

\subsubsection{Customization strategies of FSRC}

\subsubsection{Assessment of scenarios}

It is essential to evaluate similarities among scenarios in the FSRC method. One intuitive way is to find an optimal UC strategy and corresponding optimal dispatch for each scenario, and then make comparisons among them (Feng and Ryan, 2013). However, this strategy will suffer from expensive computation time when each scenario subproblem is a large MIP. Instead, measurement of the relative performance of given first-stage decisions in the second stage for each scenario may suffice to distinguish among scenarios. Because the net load is the only uncertain parameter in this model, $q_{s}$ and $T_{s}$ in the general form (4.1) - (4.5) become scenario-independent $q$ and $T$, respectively. Therefore, the Evaluate step of FSRC is expanded as follows:

\section{Customization of the Evaluate step in FSRC:}

1. Find an optimal solution $\bar{x}^{*}$ of the expected value problem

$$
\begin{gathered}
\min _{x, y} c^{\top} x+q^{\top} y \\
\text { s.t. } \quad A x=b \\
\\
T x+W y=\bar{h} \\
x \text { binary }
\end{gathered}
$$

where $\bar{h}=E\left[h_{s}\right]$.

2. Obtain optimal values of the second-stage decisions, $y_{s}^{*}$, by solving a scenario subproblem (4.17) 
for each $s \in \mathscr{S}$ given $\bar{x}^{*}$ :

$$
Q\left(\bar{x}^{*}, s\right)=\min _{y_{s}}\left\{q^{\top} y_{s} \mid W y_{s}=h_{s}-T \bar{x}^{*}\right\}
$$

\subsubsection{Definition of solution sensitivity indices: total cumulation (TC)}

Creating solution sensitivity indices follows after solving the sequence of ED problems. As in the common practice of solving a deterministic unit commitment problem with an expected net load forecast, realization of higher net loads may require higher utilization of expensive generating units and, as a direct consequence, higher production cost and possible shortages will be realized; with lower net loads, lower generation levels and production cost will result along with possible excess generation. Therefore, we use the hourly generation cost of each generator and load imbalances throughout the scheduling horizon to distinguish net load levels among scenarios, and serve as the elementary entries to create solution sensitivity indices. To avoid high dimensional data analysis, total generation cost, total excess and total shortage form the corresponding solution sensitivity indices.

Once solution sensitivity indices have been created, a clustering algorithm is applied to identify scenarios with similar sensitivity index values. Due to different effects of excess and shortage on power systems, they are weighted differently in the clustering procedure. The excess generation could be alleviated by de-committing generators; e.g., curtailing renewable energy generation, and charging storage devices, such as batteries and pumped-storage hydro plants. Shortage will require more electric power to be transmitted from other areas or even load curtailment, which could impose high costs. Therefore, shortage is assigned a higher weight in the clustering procedure.

The customization of FSRC for stochastic unit commitment is completed as follows. The basic approach (TC) is to cluster scenarios based on total cost, shortage and excess generation. A variant that pre-categorizes scenarios based on their qualitative impacts $(\mathrm{TC}+\mathrm{PC})$ is also explored.

Strategy 1. (TC)

\section{Customization of the Summarize step in FSRC:}

\section{Define}

$$
\mathscr{F}_{1}\left(\bar{x}, y_{s}^{*}\right):=\sum_{t \in \mathscr{T}} \sum_{g \in \mathscr{G}} c_{g t s}^{p}\left(\bar{p}_{g t s}^{*}\right),
$$


the total cumulative generation cost through the whole scheduling horizon over all generators for $s \in \mathscr{S}$

2. Define

$$
\mathscr{F}_{2}\left(\bar{x}, y_{s}^{*}\right):=\sum_{t \in \mathscr{T}} \sum_{b \in \mathscr{B}} \bar{\alpha}_{b t s}^{*-}
$$

and

$$
\mathscr{F}_{3}\left(\bar{x}, y_{s}^{*}\right):=\sum_{t \in \mathscr{T}} \sum_{b \in \mathscr{B}} \bar{\alpha}_{b t s}^{*+}
$$

where $\bar{p}_{g t s}^{*}, \bar{\alpha}_{b t s}^{*-}$ and $\bar{\alpha}_{b t s}^{*+}$ are from $y_{s}^{*}$.

\section{Customization of the Cluster step in FSRC:}

1. Scale each scenario generation cost to the average generation cost over all scenarios, as

$$
\hat{\mathscr{F}}_{1}:=|\mathscr{S}| \sum_{t \in \mathscr{T}} \sum_{g \in \mathscr{G}} c_{g t s}^{p}\left(\bar{p}_{g t s}^{*}\right) / \sum_{s \in \mathscr{S}} \sum_{t \in \mathscr{T}} \sum_{g \in \mathscr{G}} c_{g t s}^{p}\left(\bar{p}_{g t s}^{*}\right), \forall s \in \mathscr{S}
$$

2. Obtain the average value $\bar{\Lambda}$ of nonzero load imbalances over all scenarios

$$
\bar{\Lambda}=\frac{\sum_{s \in \mathscr{S}} \sum_{t \in \mathscr{T}} \sum_{b \in \mathscr{B}}\left(\bar{\alpha}_{b+s}^{*+}+\bar{\alpha}_{b t s}^{*-}\right)}{\left|\mathscr{A}^{+}\right|+\left|\mathscr{A}^{-}\right|}
$$

where $\mathscr{A}^{+}=\left\{\bar{\alpha}_{b t s}^{*+} \mid \bar{\alpha}_{b t s}^{*+}>0\right\}$ and $\mathscr{A}^{-}=\left\{\bar{\alpha}_{b t s}^{*-} \mid \bar{\alpha}_{b t s}^{*-}>0\right\}$. Then scale $\mathscr{F}_{2}$ and $\mathscr{F}_{3}$ as

$$
\begin{aligned}
& \hat{\mathscr{F}}_{2}:=\sum_{t \in \mathscr{T}} \sum_{b \in \mathscr{B}} \bar{\alpha}_{b t s}^{*-} / \bar{\Lambda}, \forall s \in \mathscr{S} \\
& \hat{\mathscr{F}}_{3}:=\sum_{t \in \mathscr{T}} \sum_{b \in \mathscr{B}} \bar{\alpha}_{b t s}^{*+} / \bar{\Lambda}, \forall s \in \mathscr{S} ;
\end{aligned}
$$

3. Using weights for the scaled production cost and load imbalances, form the solution sensitivity index vector $V_{s}=\left[w_{c} \hat{\mathscr{F}}_{1}, w_{+} \hat{\mathscr{F}}_{2}, w_{-} \hat{\mathscr{F}}_{3}\right]$.

4. Use the $L_{2}$ norm in the k-means method.

If a reserve requirement $R_{t}$ is specified in each hour $t$ of the scheduling horizon, the solution sensitivity indices for each scenario can be extended to include total excess and total shortage in reserve 
requirement as well, to be weighted differently in the clustering process. Although transmission constraints are not included in the following case study, the proposed scenario reduction procedure can be extended to that case by grouping buses in specified zones together, and using cumulated shortage, excess and generation cost over each group as solution sensitivity indices. The dimension of $V_{s}$ increases accordingly.

\subsubsection{Pre-categorization of scenarios in clustering (PC)}

In the basic FSRC (TC) of Section 4.4.2.2, scenarios with similar solution sensitivity indices are directly clustered into the same group by the $k$-means method. However, the computational time spent on clustering will significantly rise as the size of the whole set of scenarios and dimension of solution sensitivity indices increase. The computational complexity of a practical $k$-means method is $O(|\mathscr{S}|$ Imn $)$ (Manning et al., 2008), where $I$ is the specified number of iterations, $m$ is the dimension of the measurement vector, and $n$ is the number of clusters. In our application, $m$ represents the number of solution sensitivity indices and $n$ is the number of scenarios to select from $\mathscr{S}$. If scenario set $\mathscr{S}$ is partitioned into subsets $\mathscr{S}_{i}, i=1, \cdots, \kappa$, and only $n_{\kappa}$ scenarios are selected from each, while satisfying $\sum_{i=1}^{\kappa}\left|\mathscr{S}_{i}\right|=|\mathscr{S}|$ and $\sum_{i=1}^{\kappa} n_{\kappa}=n$, the computational complexity follows $O\left(\sum_{i=1}^{\kappa}\left|\mathscr{S}_{i}\right| n_{\kappa} I m\right)$, which will be much smaller than $O(|\mathscr{S}| \operatorname{Imn})$ if $\mathscr{S}$ is large. Therefore, implementing $k$-means within a suitably chosen partition may save computational time in FSRC.

In the creation of solution sensitivity indices, scaled load imbalance of a scenario not only shows imbalance comparisons to other scenarios, but also indicates whether a UC strategy provides sufficient generation capacity. Hence, pre-categorizing scenarios by emphasizing directions of imbalance provides a possible partition of the whole scenario set $\mathscr{S}$; i.e., existence of shortage or excess. All scenarios can be grouped into four categories: only shortage existing, only excess existing, no imbalance existing, and existence of both shortage and excess, denoted as $\mathscr{M}_{i}$, with $i \in \mathfrak{C}=\{+,-, o, \pm\}$, respectively.

Following this categorization, it is necessary to identify the number of scenarios to be selected from each category. In this paper, we aim to match the frequency with which each category occurs in the whole scenario set. For each category $\mathscr{M}_{i}$, let $\varepsilon_{i}=\sum_{s \in \mathscr{M}_{i}} \xi_{s}$ be the total probability of scenarios in category $\mathscr{M}_{i}$. The following model assigns a number of selected scenarios, out of $n$ total, to each 
category.

$$
\begin{gathered}
\min _{z_{i}} \sum_{i \in \mathfrak{C}}\left|z_{i}-n \cdot \varepsilon_{i}\right| \\
\text { s.t. } \\
\sum_{i \in \mathfrak{C}} z_{i}=n \\
z_{i} \geq 1, \forall i \in \mathscr{J} \\
z_{i} \in \mathbb{Z}_{0}^{+}, \forall i \in \mathfrak{C}
\end{gathered}
$$

Here, $z_{i}$ denotes the number of selected scenarios from category $\mathscr{M}_{i}$ and $\mathscr{J}=\left\{i \in \mathfrak{C} \mid \varepsilon_{i}>0\right\}$ represents the categories represented in the original scenario set. Formula (4.20) requires that at least one scenario is selected from every nonempty category, which avoids ignoring categories with small probabilities, and in a certain way takes into account extreme scenarios. Notice that (4.18) - (4.21) is a simple mixed integer program which will not cost much computational time in general. Therefore, it is possible to save computational time of FSRC if pre-categorization of scenarios is applied to customize the Cluster step in FSRC.

Fig. 4.1 summarizes the scenario reduction procedure of the customized FSRC method. Note that pre-categorization of scenarios is an optional step during the clustering phase.

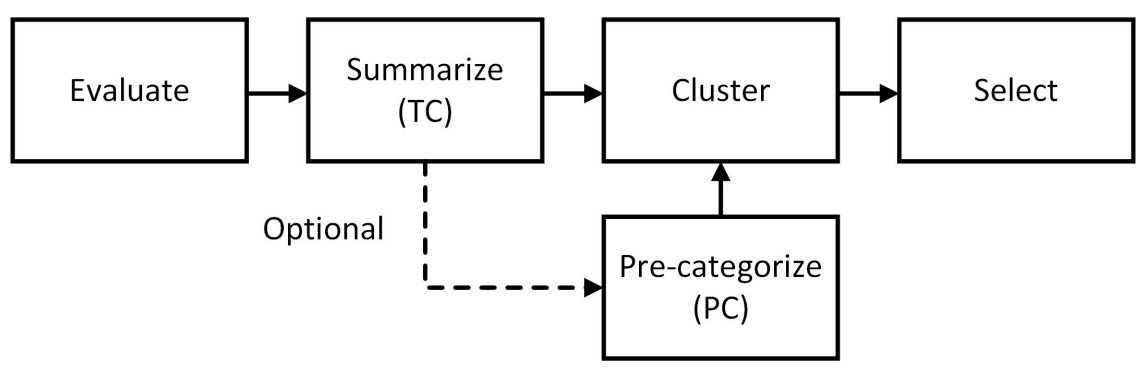

Figure 4.1 Scenario reduction procedure of customized FSRC method

\subsubsection{Evaluation of selected scenarios}

Instead of comparing similarity in the distributions between selected scenarios and the whole set of scenarios, we evaluate selected sets of scenarios by investigating the performance of the resulting UC schedules against the whole set of scenarios Kaut and Wallace (2007). 
For a scenario subset $\mathscr{S}^{\prime} \subseteq \mathscr{S}$, the evaluation procedure is given as below, where we use prime superscripts to denote quantities obtained from solving the problem with $\mathscr{S}^{\prime}$ and asterisks to denote corresponding quantities associated with $\mathscr{S}$ :

1. Find $f\left(\mathscr{S}^{\prime}\right)$ as in (4.1) and a corresponding optimal first-stage decision vector $x^{\prime}$. Extract $v_{g t}^{\prime}$ from $x^{\prime}$.

2. Evaluate $\mathscr{Q}\left(x^{\prime}, \mathscr{S}\right)$ as in (4.4), and obtain $p_{g t s}^{\prime}, \alpha_{b t s}^{\prime+}$ and $\alpha_{b t s}^{\prime-}, \forall g \in \mathscr{G}, \forall t \in \mathscr{T}, \forall s \in \mathscr{S}$.

3. Find $f(\mathscr{S})$ and a corresponding optimal $x^{*}$. Extract $v_{g t}^{*}$ from $x^{*}$, and obtain $p_{g t s}^{*}, \alpha_{b t s}^{*+}$ and $\alpha_{b t s}^{*-}$, $\forall g \in \mathscr{G}, \forall t \in \mathscr{T}, s \in \mathscr{S}$ simultaneously.

4. Compare $x^{\prime}$ to $x^{*}$, and performance measures $\mathscr{U}\left(\mathscr{S}^{\prime}\right)$ to $\mathscr{U}(\mathscr{S}), \Phi\left(\mathscr{S} \mid \mathscr{S}^{\prime}\right)$ to $\Phi(\mathscr{S} \mid \mathscr{S}), \Psi^{+}\left(\mathscr{S} \mid \mathscr{S}^{\prime}\right)$ to $\Psi^{+}(\mathscr{S} \mid \mathscr{S})$ and $\Psi^{-}\left(\mathscr{S} \mid \mathscr{S}^{\prime}\right)$ to $\Psi^{-}(\mathscr{S} \mid \mathscr{S})$.

Definitions of $\mathscr{U}\left(\mathscr{S}^{\prime}\right), \Phi\left(\mathscr{S} \mid \mathscr{S}^{\prime}\right), \Psi^{+}\left(\mathscr{S} \mid \mathscr{S}^{\prime}\right)$ and $\Psi^{-}\left(\mathscr{S} \mid \mathscr{S}^{\prime}\right)$ follow:

\section{Commitment cost}

$$
\mathscr{U}\left(\mathscr{S}^{\prime}\right)=\sum_{t \in \mathscr{T}} \sum_{g \in \mathscr{G}} c_{g t}^{u}\left(v_{g t}^{\prime}\right)
$$

\section{Expected generation cost against scenario set $\mathscr{S}$}

$$
\Phi\left(\mathscr{S} \mid \mathscr{S}^{\prime}\right)=\sum_{s \in \mathscr{S}} \sum_{t \in \mathscr{T}} \sum_{g \in \mathscr{G}} \xi_{s} c_{g t s}^{p}\left(p_{g t s}^{\prime}\right)
$$

\section{Expected shortage and excess against scenario set $\mathscr{S}$}

$$
\begin{aligned}
& \Psi^{+}\left(\mathscr{S} \mid \mathscr{S}^{\prime}\right)=\sum_{s \in \mathscr{S}} \sum_{t \in \mathscr{T}} \sum_{b \in \mathscr{B}} \xi_{s} \alpha_{b t s}^{++} \\
& \Psi^{-}\left(\mathscr{S} \mid \mathscr{S}^{\prime}\right)=\sum_{s \in \mathscr{S}} \sum_{t \in \mathscr{T}} \sum_{b \in \mathscr{B}} \xi_{s} \alpha_{b t s}^{\prime-}
\end{aligned}
$$

The measures $\mathscr{U}(\mathscr{S}), \Phi(\mathscr{S} \mid \mathscr{S}), \Psi^{+}(\mathscr{S} \mid \mathscr{S})$ and $\Psi^{-}(\mathscr{S} \mid \mathscr{S})$ are obtained by substituting $v_{g t}^{*}, p_{\text {gts }}^{*}$, $\alpha_{b t s}^{*+}$ and $\alpha_{b t s}^{*-}$ in (4.22) - (4.25). 


\subsection{Case studies}

The customized FSRC methods are applied to test systems down-sampled from the Independent System Operator of New England (ISO-NE). All 8 load zones in ISO-NE were treated as a single bus in the case studies. To focus on uncertainty associated with net load, outages of transmission and thermal units were not modeled in the case studies, and the associated reserve requirements also were omitted. This section is organized as follows. Section 4.5.1 briefly describes how net load scenarios were generated. To compare results between FSRC and FFS, we solved single-day SRUC problems on a sample of days from each season as reported in Section 4.5.2. Section 4.5.3 further investigates the quality of the scenario sets obtained by FSRC by solving SRUC on a rolling basis for both the selected scenarios and the whole set of original scenarios throughout a week, and comparing their solutions. All case studies of SRUC were solved in their extensive forms by PySP (Watson et al., 2012; Sandia National Laboratories, 2013b) using CPLEX in Windows on a Dell desktop with 8GB memory.

Because of the RAM limitation, subsets of 50 generators are selected from the whole fleet of over 300 generators for the single-day SRUC problems, and 20 generators for SRUC on a rolling horizon basis, to keep computation manageable. In addition, the 10 highest probability wind energy scenarios are selected from 50 wind energy scenarios to cross with 8 load scenarios which have been generated as described in (Feng et al., 2014), forming 80 hourly net load scenarios. The net load scenarios were scaled down to match the reduced generation capacity in both case studies. As discussed in Section

4.4.2, penalties on shortage and excess were initially set to $10^{7} \$ / \mathrm{MWh}$ and $10^{5} \$ / \mathrm{MWh}$ respectively, which are four and two, respectively, orders of magnitude larger than the marginal cost of the most expensive unit, to avoid shortage and excess and emphasize the negative impacts of shortage. In addition, the weights $w_{c}, w_{+}$and $w_{-}$were set to $0.3,0.4$ and 0.3 , respectively, to strengthen the emphasis on shortage in clustering.

\subsubsection{Scenario generation}

Load scenario generation in this paper started from a historical database of day-ahead hourly weather forecast and corresponding actual hourly load sequences in 2011 in ISO-NE (ISO New England, 2013b). Date ranges were identified first to group days according to similarity of the relationship be- 
tween hourly weather and load, forming "seasons." This identification of seasons accounted for ad hoc characterizations such as diurnal lighting patterns, heating vs. cooling by using air conditioning, and sociological factors including holiday lighting and school being in session or not. In each season, transformations were performed to aggregate data across days of the week and geographic zones. Days in each season were then segmented according to temperature forecast bands. The relationship between hourly loads and weather forecast variables over a day were approximated by a nonparametric regression function and distributions of hourly residuals were approximated as well. Having identified the regression functions and hourly error distributions for each hour in each segment, scenarios were generated as follows, for a given day $D$ (Feng et al., 2014):

1. Identify the season to which day $D$ belongs and the segment to which its weather forecast generated on day $D-1$ belongs.

2. Apply the approximated regression function to the weather forecast to get a time-series forecast, and generate the desired number of load scenarios by approximating the distributions of the forecast errors.

3. Invert the transformations to match the day of the week and geographic zone.

Hourly wind scenarios were obtained from a commercial vendor (3TIER Inc., 2012) according to an analogue method (Mahoney et al., 2011). These scenarios were designed to represent a future representing 20\% penetration of wind energy in the eastern U.S. in 2024 (Corbus et al., 2011). Generated load scenarios for 2011 were scaled by the $2.27 \%$ increase per year Corbus et al. (2011) to approximate demand levels in 2024. Wind energy was assumed to be nondispatchable, and thereby considered as negative load in this paper. The net load scenarios representing demands in model (4.33)-(4.58) were obtained by subtracting wind energy from load in crossed sets of scenarios.

\subsubsection{Independent daily SRUC}

A typical summer week, ranging from 2011-07-10 to 2011-07-16, is selected to test customized FSRC in independent daily SRUC first, and several days are randomly selected from other seasons for testing as well. In independent daily SRUC, the initial status of each generating unit on day $D$ is 
independent of its status in the last period on day $D-1$. The initial conditions are identified by solving an ED problem for the initial period in which each unit is set on and demand is the expected value for that period over all scenarios. All units for which generation levels are higher than corresponding minimum output, $\underline{P}_{g}$, will be set on, and initial generation levels will be the values derived from the expected value ED problem. To investigate how FSRC performs, subsets of 10, 20, 30, 40, 50 and 60 scenarios are selected from the $|\mathscr{S}|=80$ net load scenarios by customized FSRC and FFS methods separately. For illustration, the whole set of net load scenarios on day 2011-07-11 are displayed in Fig. 4.2, and 20 scenarios selected by FFS on the same day are shown in Fig. 4.3. Assessment of selected scenarios follows the procedure described in Section 4.4.3.

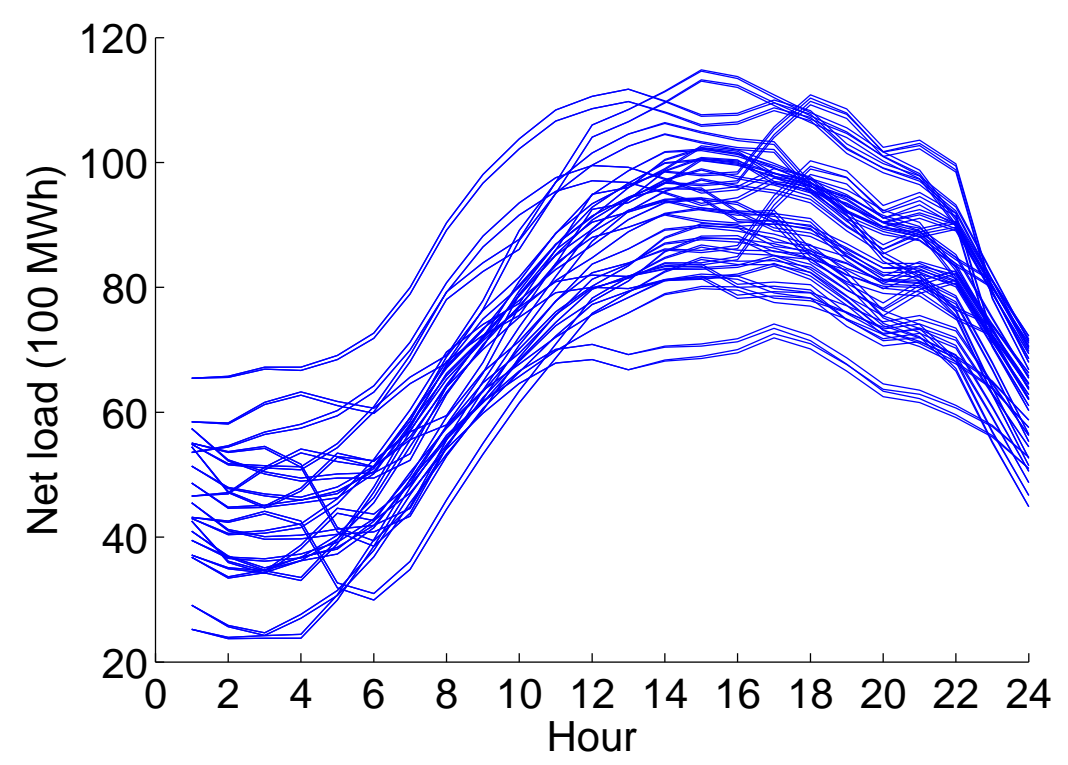

Figure 4.2 80 net load scenarios on 2011-07-11

Section 4.5.2.1 provides numerical results of reduced scenarios resulting from solution sensitivity indices created by TC. Section 4.5.2.2 displays scenario reduction effects of FSRC when the optional PC step is included.

\subsubsection{Independent daily SRUC: applying TC}

The subset of 20 scenarios on 2011-07-11 selected by FSRC with strategy TC is displayed in Fig. 4.4. Comparisons between Figs. 4.3 and 4.4 suggest that scenarios selected by applying TC in 


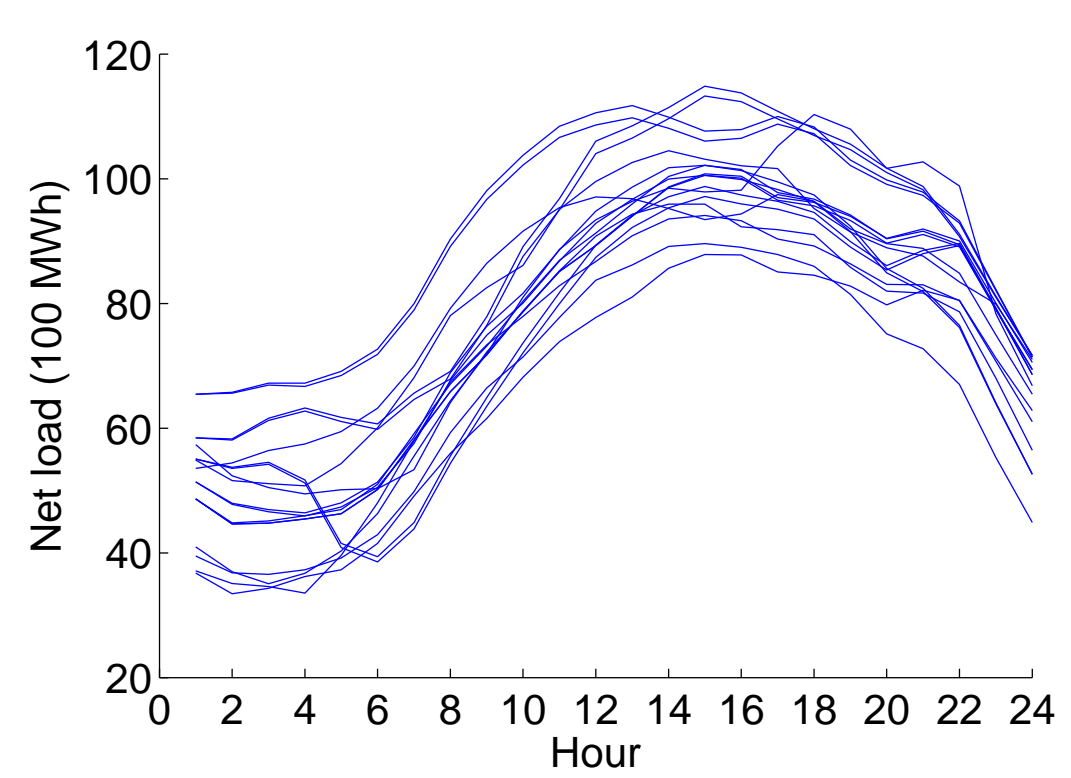

Figure 4.3 20 selected scenarios by FFS on 2011-07-11

FSRC have a wider range than those from FFS, which may indicate that more extreme situations have been retained by FSRC.

For convenience in describing the comparisons of scenario subsets obtained by different methods, $\mathscr{S}_{F F S}^{\prime}$ denotes a subset of scenarios selected by FFS, and $\mathscr{S}_{F S R C}^{\prime}$ a subset of scenarios selected by FSRC. Notations $\mathscr{S}_{F S R C: T C}^{\prime}$ and $\mathscr{S}_{F S R C: T C+P C}^{\prime}$ further distinguish the two variants of FSRC.

Scenario subsets are evaluated following the procedure in Section 4.4.3. Given the whole set of scenarios $\mathscr{S}$, the commitment cost, $\mathscr{U}\left(\mathscr{S}_{F F S}^{\prime}\right)$ and $\mathscr{U}\left(\mathscr{S}_{F S R C: T C}^{\prime}\right)$, and expected generation costs, $\Phi\left(\mathscr{S} \mid \mathscr{S}_{F F S}^{\prime}\right)$ and $\Phi\left(\mathscr{S} \mid \mathscr{S}_{F S R C: T C}^{\prime}\right)$, are accumulated through the week and displayed in Fig. 4.5 for each cardinality, $n$, of the selected scenario sets. Deviations from the optimal shortage, $\Psi^{+}\left(\mathscr{S} \mid \mathscr{S}_{F F S}^{\prime}\right)-$ $\Psi^{+}(\mathscr{S} \mid \mathscr{S})$ and $\Psi^{+}\left(\mathscr{S} \mid \mathscr{S}_{F S R C: T C}^{\prime}\right)-\Psi^{+}(\mathscr{S} \mid \mathscr{S})$, and deviation from optimal excess, $\Psi^{-}\left(\mathscr{S} \mid \mathscr{S}_{F F S}^{\prime}\right)-$ $\Psi^{-}(\mathscr{S} \mid \mathscr{S})$ and $\Psi^{-}\left(\mathscr{S} \mid \mathscr{S}_{F S R C: T C}^{\prime}\right)-\Psi^{-}(\mathscr{S} \mid \mathscr{S})$, of FFS and FSRC with TC over the summer week are displayed in Fig. 4.6 for each cardinality $n$, as well. Fig. 4.5 shows that, when evaluated over the whole scenario set, commitments from FSRC with the TC strategy and FFS result in similar cost over the summer week for each $n$. However, the FSRC method causes less expected shortage while resulting in similar levels of excess, as shown in Fig. 4.6. Fig. 4.6 also illustrates that the expected shortage, $\Psi^{+}\left(\mathscr{S} \mid \mathscr{S}_{F S R C}^{\prime}\right)$, result from FSRC will better approximate $\Psi^{+}(\mathscr{S} \mid \mathscr{S})$ as the cardinality, $n$, increases 


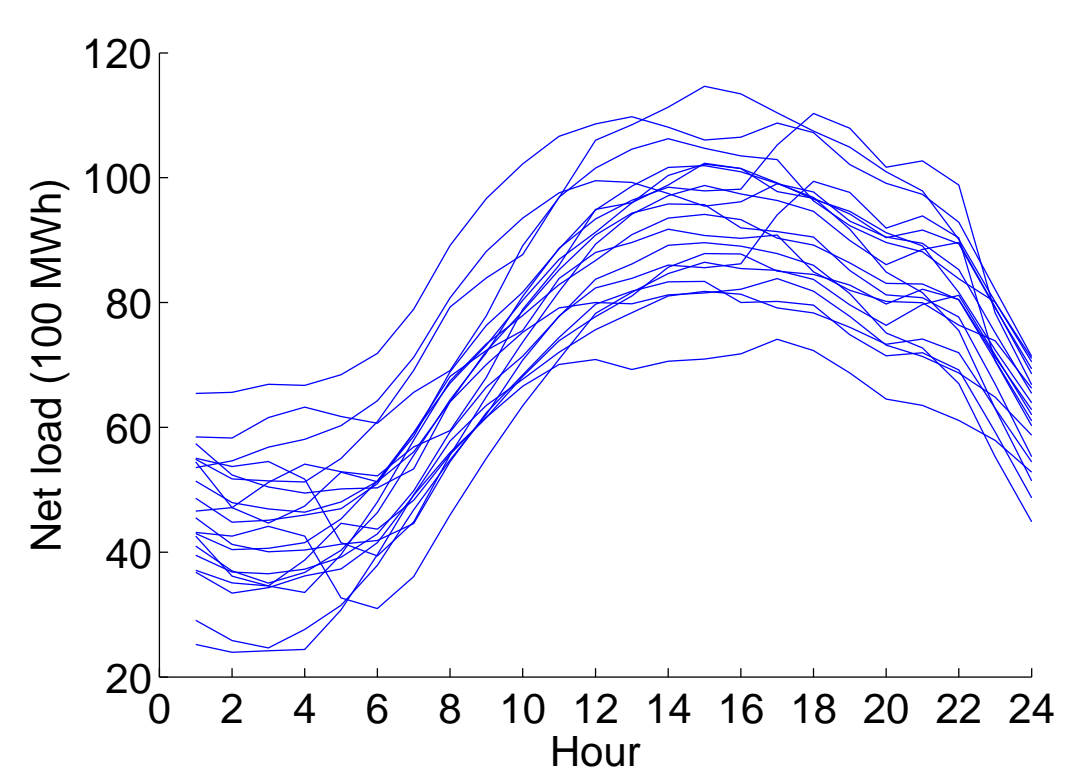

Figure 4.4 20 selected scenarios by FSRC: TC on 2011-07-11

from 10 to 60.

\subsubsection{Independent daily SRUC: applying TC and PC}

Pre-categorizing scenarios before clustering is applied to customize FSRC in this section. For comparison, 20 scenarios selected by FSRC with TC and PC on 2011-07-11 are displayed in Fig. 4.7. Only minor differences can be observed between the sets of selected scenarios shown in Fig. 4.4 and Fig.4.7.

Similar to Section 4.5.2.1, commitment cost, $\mathscr{U}\left(S_{F F S}^{\prime}\right)$ and $\mathscr{U}\left(S_{F S R C: T C+P C}^{\prime}\right)$, expected generation cost, $\Phi\left(\mathscr{S} \mid \mathscr{S}_{F F S}^{\prime}\right)$ and $\Phi\left(\mathscr{S} \mid \mathscr{S}_{F S R C: T C+P C}^{\prime}\right)$, expected shortage, $\Psi^{+}\left(\mathscr{S} \mid \mathscr{S}_{F F S}^{\prime}\right)$ and $\Psi^{+}\left(\mathscr{S} \mid \mathscr{S}_{F S R C: T C+P C}^{\prime}\right)$, and expected excess, $\Psi^{-}\left(\mathscr{S} \mid \mathscr{S}_{F F S}^{\prime}\right)$ and $\Psi^{-}\left(\mathscr{S} \mid \mathscr{S}_{F S R C: T C+P C}^{\prime}\right)$, are accumulated separately through the week, and their comparisons are displayed in Figs. 4.5 and 4.6. From Fig. 4.5, applying TC and PC in FSRC will also result in similar commitment and expected generation cost to their counterparts from FFS for different $n$. But Fig. 4.6 shows that FSRC with TC and PC results in less shortage while yielding not much more excess generation, especially when the reduction is substantial.

According to these results, the FSRC methods result in less shortage and more economical unit commitments than FFS if a small set of scenarios are selected from the whole set; i.e., fewer than $25 \%$ of the whole set of scenarios are applied to obtain the UC strategy. The performances of FFS and FSRC are 


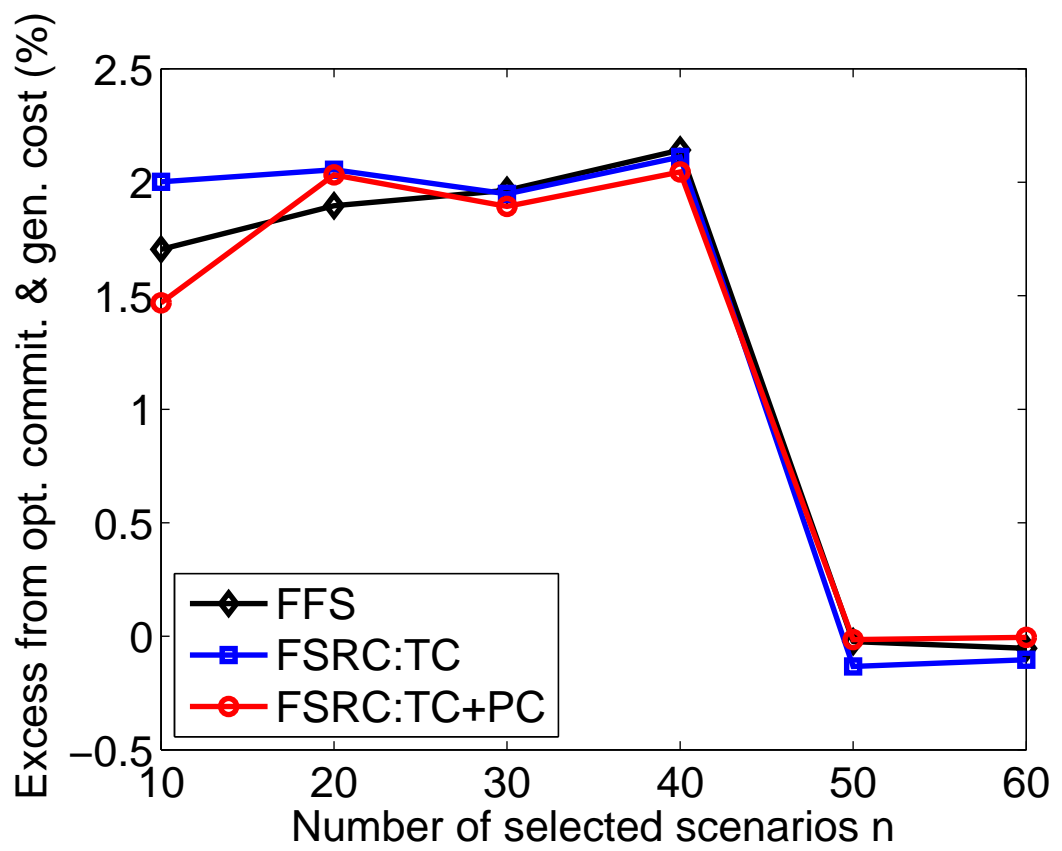

Figure 4.5 Deviations from optimal commitment and generation cost of FSRC and FFS through the summer week, 2011-07-10 to 2011-07-16, for different cardinality $n$

more similar when the cardinality of the selected set is larger (greater than $40 \%$ of the total scenarios). To assess the performance of selected scenarios especially when $n$ is small, 20 scenarios were selected from the whole set in randomly selected days in spring, fall and winter to run independent daily SRUC. Fig. 4.8 shows the relative differences in commitment and expected generation cost between FFS and FSRC:

$$
\frac{\mathscr{U}\left(\mathscr{S}_{F F S}^{\prime}\right)+\Phi\left(\mathscr{S} \mid \mathscr{S}_{F F S}^{\prime}\right)-\mathscr{U}\left(\mathscr{S}_{F S R C}^{\prime}\right)-\Phi\left(\mathscr{S} \mid \mathscr{S}_{F S R C}^{\prime}\right)}{\mathscr{U}\left(\mathscr{S}_{F F S}^{\prime}\right)+\Phi\left(\mathscr{S} \mid \mathscr{S}_{F F S}^{\prime}\right)} \times 100 \%
$$

for each selected day. The small percentages displayed indicate that scenarios selected by both FSRC variants result in similar costs to those that result from scenarios selected by FFS.

Fig. 4.9 and Fig. 4.10 contrast solutions optimized using scenarios selected by FFS and FSRC by showing $\Psi^{+}\left(\mathscr{S} \mid \mathscr{S}_{F F S}^{\prime}\right)-\Psi^{+}\left(\mathscr{S} \mid \mathscr{S}_{F S R C}^{\prime}\right)$ and $\Psi^{-}\left(\mathscr{S} \mid \mathscr{S}_{F F S}^{\prime}\right)-\Psi^{-}\left(\mathscr{S} \mid \mathscr{S}_{F S R C}^{\prime}\right)$, respectively. Fig. 4.9 illustrates that both of the FSRC variants usually result in less shortage than FFS in the selected days, while providing similar excess amounts on all but two days (in Fig. 4.10). The results taken together indicate that FSRC results in more conservative solutions than FFS; i.e., more generation capacity is scheduled to be available. This is consistent with the smaller penalties applied to excess generation than to shortage. 

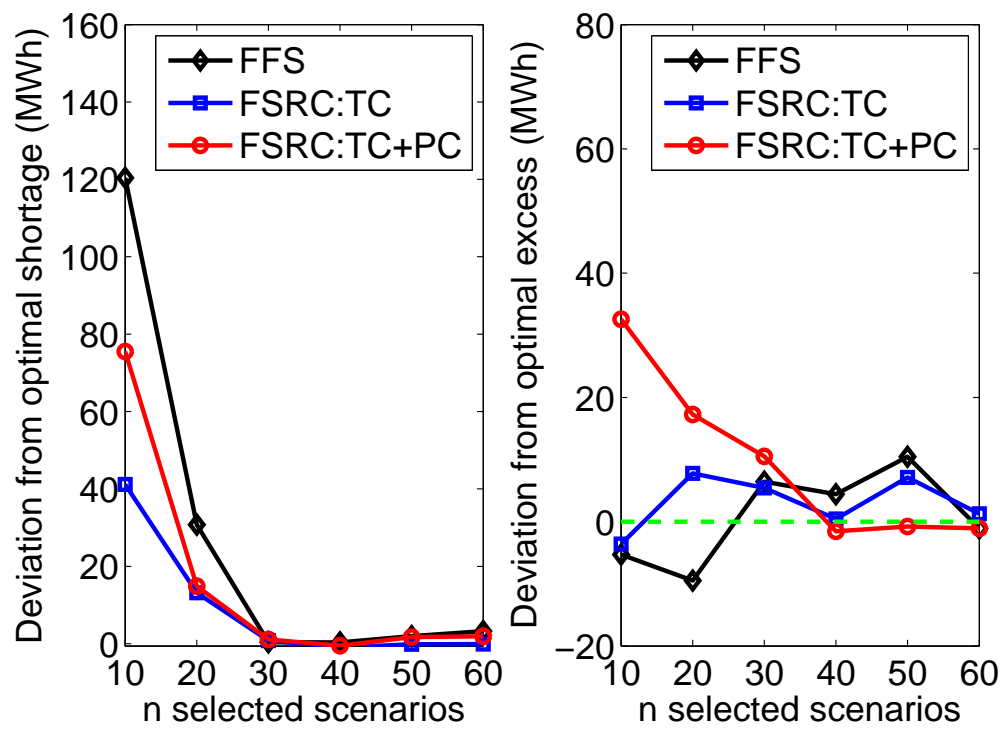

Figure 4.6 Deviation from optimal load imbalance of FSRC and FFS through the summer week, 2011-07-10 to 2011-07-16, for different cardinality $n$

To investigate the clustering and selection time demands of FSRC:TC and FSRC:TC+PC, more load scenarios (27 load scenarios) were generated by the same procedure (Feng et al., 2014) for 12 selected days in 2011 (2011-6-22, 2011-7-19 and 2011-8-13 in summer and all days in Fig. 4.10), and then crossed with their corresponding 50 wind energy scenarios to form 1350 net load scenarios. Because of physical RAM limitations, UC strategies are not computed for 1350 -scenarios cases but only clustering time and selection time of FSRC are displayed in Fig. 4.11. The computational time of FSRC is significantly decreased if pre-categorization is applied in the Cluster step especially for large $n$. The time savings is approximately $80 \%$ when 540 scenarios are selected out of 1350 net load scenarios.

To investigate the sensitivity of FSRC to the penalty parameters used in clustering, different pairs of these parameters were tested. These studies were done for $n=20$ because performance of FSRC differs more from FFS when selecting smaller subsets. Fig. 4.12 displays expected savings in shortage, $\Psi^{+}\left(\mathscr{S} \mid \mathscr{S}_{F F S}^{\prime}\right)-\Psi^{+}\left(\mathscr{S} \mid \mathscr{S}_{F S R C}^{\prime}\right)$, and excess $\Psi^{-}\left(\mathscr{S} \mid \mathscr{S}_{F F S}^{\prime}\right)-\Psi^{-}\left(\mathscr{S} \mid \mathscr{S}_{F S R C}^{\prime}\right)$ of FSRC in the summer week which ranges from 2011-07-10 to 2011-07-16. In Fig. 4.12, UC schedules obtained from both of the FSRC variants result in lower levels of shortage than the schedule obtained from FFS. Moreover, both of FSRC variants lead to similar levels of shortage and excess through the pairs of penalties. Overall, the numerical results for the independent daily SRUC testing indicate that the FSRC methods 


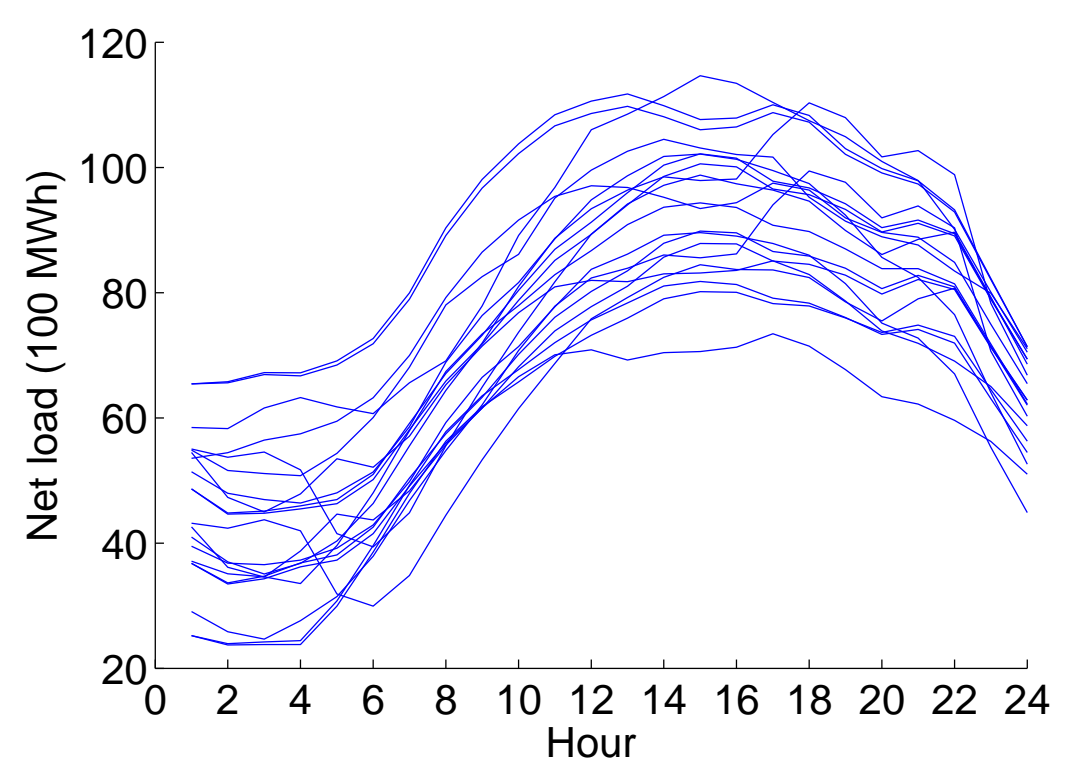

Figure 4.7 20 Selected scenarios by FSRC: TC+PC on 2011-07-11

can result in more economical and reliable schedules compared to FFS.

\subsubsection{Rolling horizon SRUC over a week}

Rolling horizon SRUC is performed in the same summer week as tested in Section 4.5.2. The rolling horizon procedure starts by solving SRUC over 36 hours on days $D$ and $D+1$, where net load values in a scenario from hour 25 to hour 36 are duplicated from hour 1 to hour 12 in the same scenario. This extension of the daily planning horizon avoids the shut-down of units toward the end of the day that might otherwise occur due to end-of-study effects. The commitment states of units at hour 24 on day $D$ are adopted as initial states of units on day $D+1$, and the initial generation level (relevant to ramping constraints) of each unit for day $D+1$ is set to its expected generation over all scenarios at hour 24 of day $D$. The next two sections describe the performance of the two variants of FSRC in selecting 40 scenarios from the total of 80 for each day. The UC schedules obtained by optimizing with the reduced sets are evaluated against all scenarios. As in Section 4.5.2, for scenario subset $\mathscr{S}^{\prime} \subseteq \mathscr{S}, \mathscr{U}\left(\mathscr{S}^{\prime}\right), \Phi\left(\mathscr{S} \mid \mathscr{S}^{\prime}\right)$, $\Psi^{+}\left(\mathscr{S} \mid \mathscr{S}^{\prime}\right)$ and $\Psi^{-}\left(\mathscr{S} \mid \mathscr{S}^{\prime}\right)$ are compared to their counterparts if the whole set of scenarios $\mathscr{S}$ are used in SRUC. Note that, to evaluate the selected scenarios in rolling SRUC, $\mathscr{T}=\{1, \cdots, 24\}$ for each day $D$ in formulas (4.22) - (4.25). Table 4.1 shows the commitment cost $\mathscr{U}(\mathscr{S})$, expected generation 


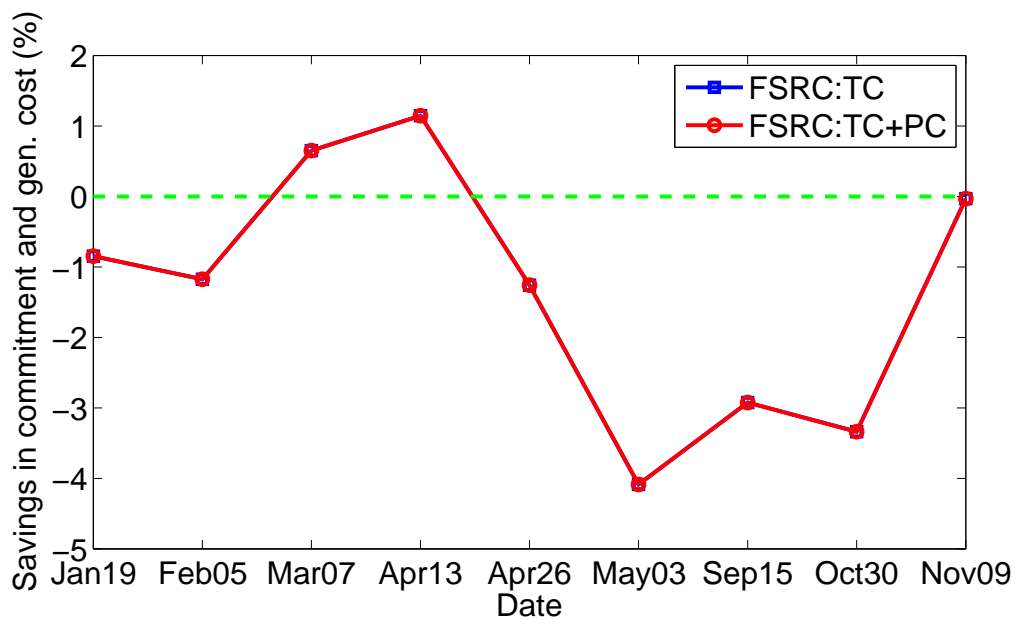

Figure 4.8 Savings in commitment and generation cost of customized FSRC methods from FFS in evaluation, $n=20$

$\operatorname{cost} \Phi(\mathscr{S} \mid \mathscr{S})$, expected shortage $\Psi^{+}(\mathscr{S} \mid \mathscr{S})$ and excess $\Psi^{-}(\mathscr{S} \mid \mathscr{S})$ evaluated for each day during the week.

Table 4.1 Expected values of the all-scenarios based SRUC through a week, 2011-07-10 to 2011-07-16, $n=80$

\begin{tabular}{lrrrr}
\hline Date & $\mathscr{U}(\mathscr{S})(\mathrm{K} \$)$ & $\Phi(\mathscr{S} \mid \mathscr{S})(\mathrm{K} \$)$ & $\Psi^{+}(\mathscr{S} \mid \mathscr{S})(\mathrm{MWh})$ & $\Psi^{-}(\mathscr{S} \mid \mathscr{S})(\mathrm{MWh})$ \\
\hline & & & & \\
$2011-07-10$ & 15 & 712 & 0 & 1288 \\
$2011-07-11$ & 27 & 943 & 96 & 367 \\
$2011-07-12$ & 32 & 873 & 13 & 341 \\
$2011-07-13$ & 43 & 1522 & 318 & 37 \\
$2011-07-14$ & 26 & 912 & 0 & 0 \\
$2011-07-15$ & 32 & 1309 & 3 & 90 \\
$2011-07-16$ & 43 & 1220 & 431 & 0 \\
Total & 216 & 7500 & & 2124 \\
\hline
\end{tabular}

\subsubsection{Rolling horizon SRUC: applying TC}

Although the scheduling horizon used in the rolling horizon test includes 36 hours, solution sensitivity indices are computed over 24 hours to select scenarios for each day. Table 4.2 summarizes the evaluated daily commitment cost, expected generation cost, and expected load imbalance through the summer week when the number of selected scenarios is $n=40$. Compared to results from the com- 


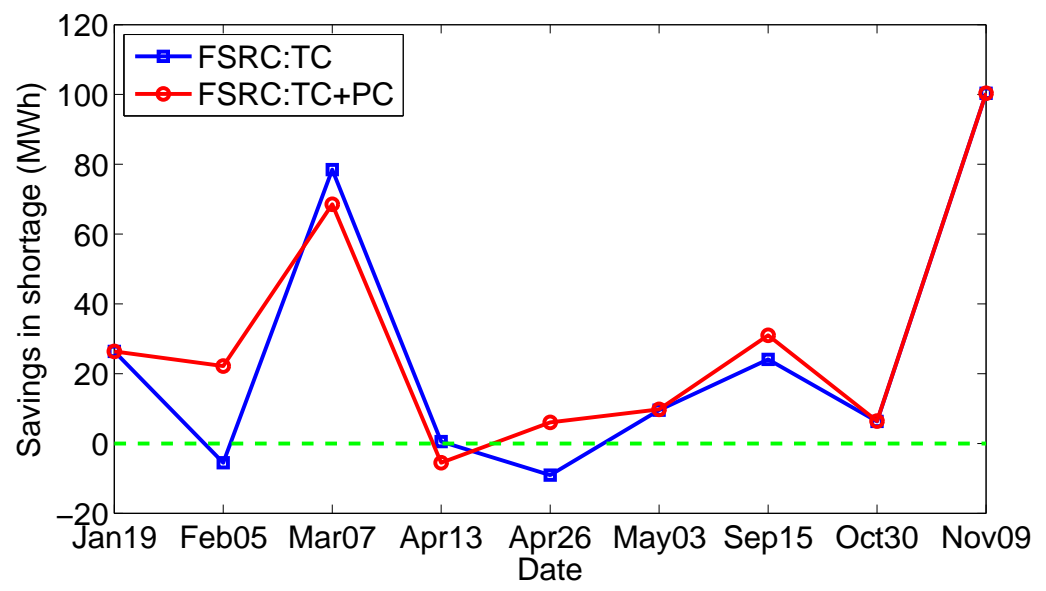

Figure 4.9 Savings in shortage of FSRC from FFS in evaluations, $n=20$

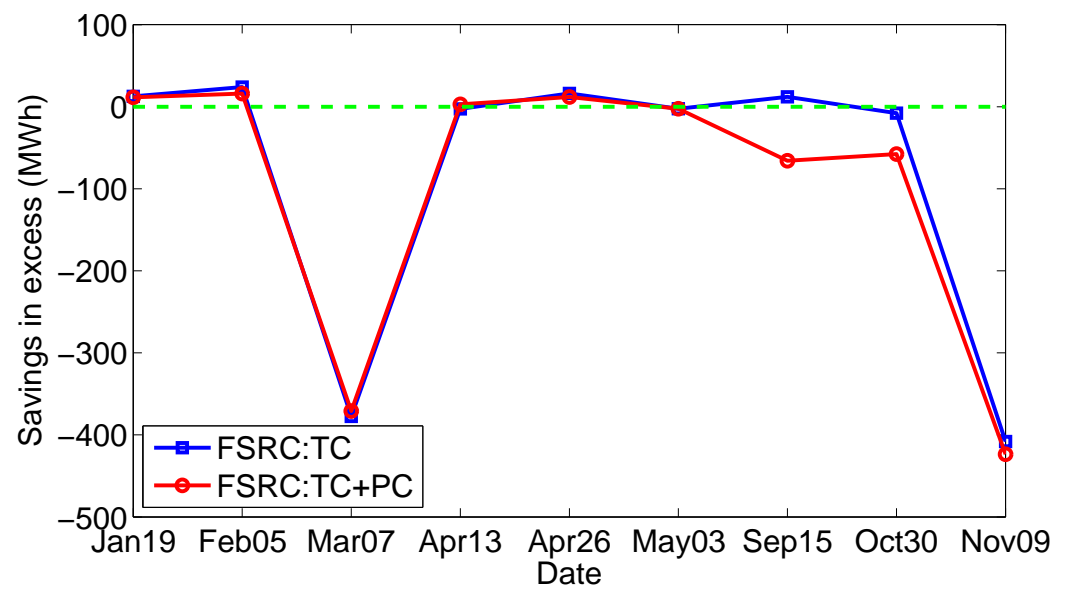

Figure 4.10 Savings in excess of FSRC from FFS in evaluations, $n=20$ 


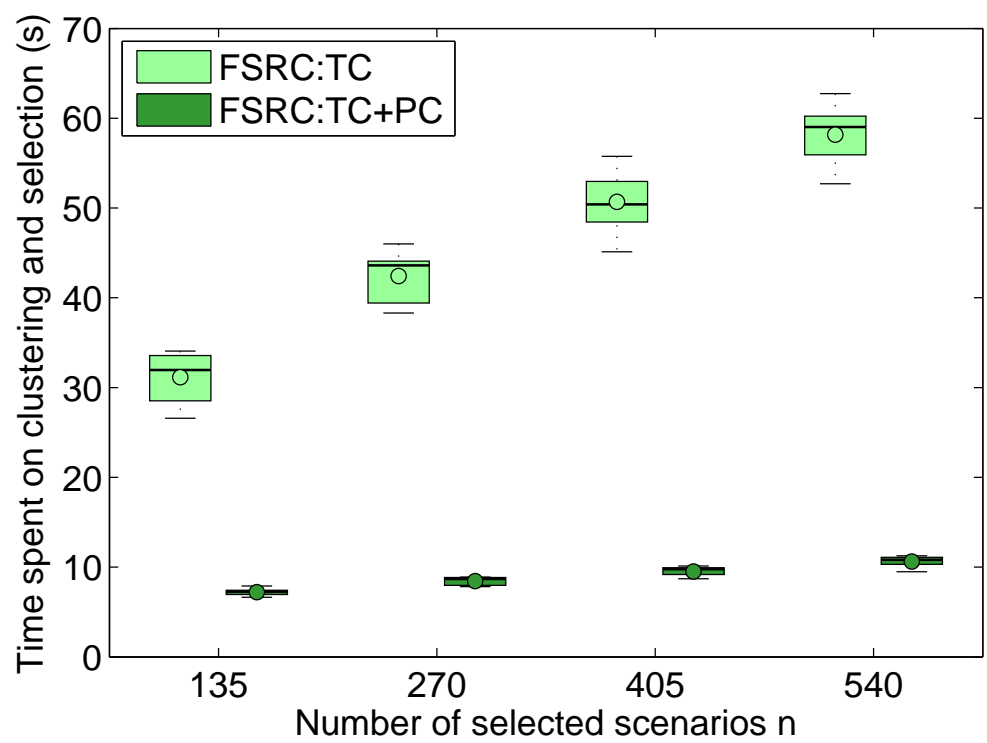

Figure 4.11 Time spent on scenario clustering and selection by FSRC for different numbers, $n$, of scenarios selected from 1350. The bar marker in each box represents the median and the round marker denotes the mean time over 12 randomly selected days in 2011.
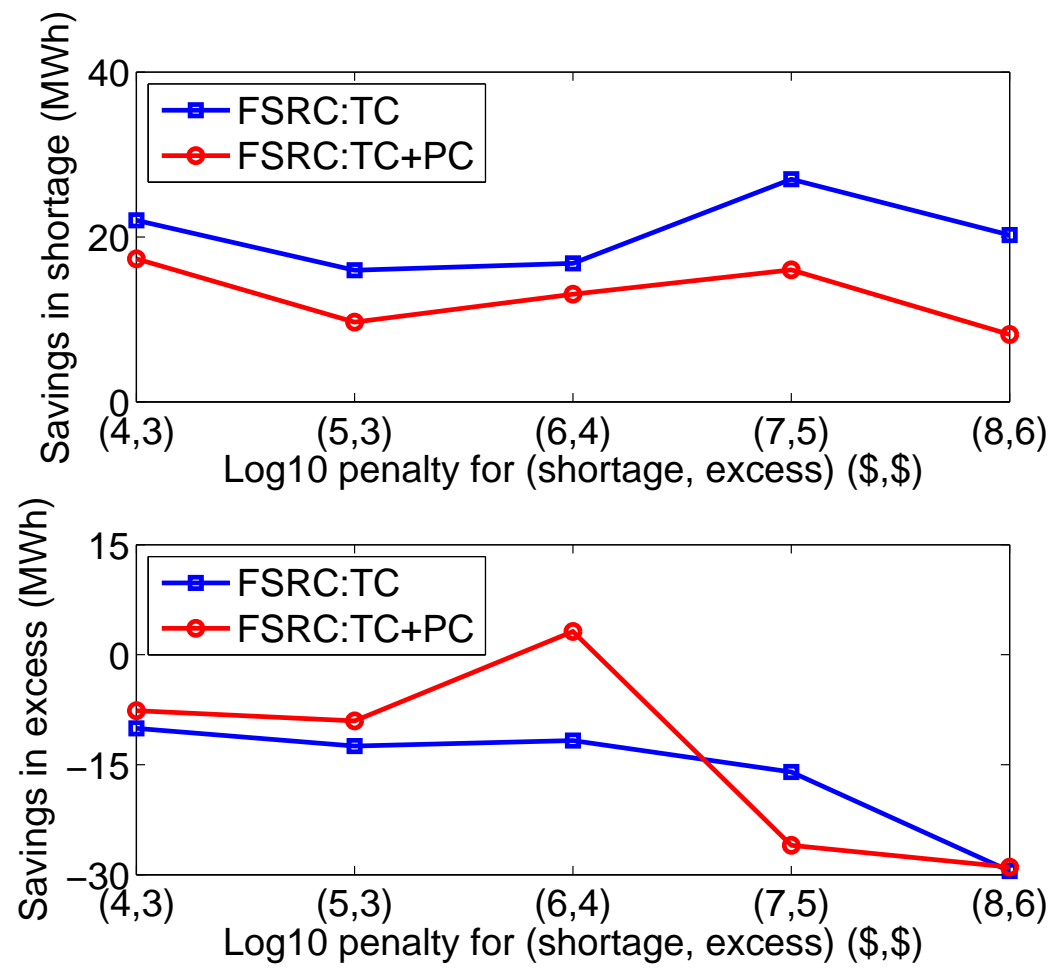

Figure 4.12 Expected savings in load imbalance of FSRC methods from FFS for 20 selected scenarios through different pairs of penalties for the week 2011-07-10 to 2011-07-16 
mitment schedule optimized over all 80 scenarios, entries in Table 4.2 correspond closely to those in Table 4.1. Thus, applying TC only in FSRC can yield an acceptable UC strategy even when half of the scenarios are ignored in the optimization.

Table 4.2 Expected values of UC from TC with respect to all scenarios through a week, 2011-07-10 to 2011-07-16, $n=40$

\begin{tabular}{lrrrr}
\hline Date & $\mathscr{U}\left(\mathscr{S}^{\prime}\right)(\mathrm{K} \$)$ & $\Phi\left(\mathscr{S} \mid \mathscr{S}^{\prime}\right)(\mathrm{K} \$)$ & $\Psi^{+}\left(\mathscr{S} \mid \mathscr{S}^{\prime}\right)(\mathrm{MWh})$ & $\Psi^{-}\left(\mathscr{S} \mid \mathscr{S}^{\prime}\right)(\mathrm{MWh})$ \\
\hline & & & & \\
$2011-07-10$ & 15 & 711 & 0 & 1289 \\
$2011-07-11$ & 27 & 965 & 96 & 361 \\
$2011-07-12$ & 31 & 873 & 15 & 335 \\
$2011-07-13$ & 44 & 1531 & 318 & 36 \\
$2011-07-14$ & 27 & 909 & 1 & 0 \\
$2011-07-15$ & 31 & 1249 & 4 & 94 \\
$2011-07-16$ & 43 & 1218 & 434 & 2115 \\
Total & 217 & 7456 & & 0 \\
\hline
\end{tabular}




\subsubsection{Rolling horizon SRUC: applying TC and PC}

Like Table 4.2, Table 4.3 shows the evaluation results of applying PC together with TC in FSRC when the cardinality of selected scenarios $n=40$. Similar values to those in Table 4.1 support the use of this variant. Comparisons between Tables 4.2 and 4.3 show that both FSRC variants result in similar commitment costs as well as expected generation costs, excess generation and shortage across the whole set of scenarios to those from the solution found with all scenarios.

Table 4.3 Expected values of UC from TC+PC with respect to all scenarios through a week, 2011-07-10 to 2011-07-16, $n=40$

\begin{tabular}{lrrrr}
\hline Date & $\mathscr{U}\left(\mathscr{S}^{\prime}\right)(\mathrm{K} \$)$ & $\Phi\left(\mathscr{S} \mid \mathscr{S}^{\prime}\right)(\mathrm{K} \$)$ & $\Psi^{+}\left(\mathscr{S} \mid \mathscr{S}^{\prime}\right)(\mathrm{MWh})$ & $\Psi^{-}\left(\mathscr{S} \mid \mathscr{S}^{\prime}\right)(\mathrm{MWh})$ \\
\hline & & & & \\
$2011-07-10$ & 15 & 712 & 0 & 1288 \\
$2011-07-11$ & 26 & 972 & 96 & 360 \\
$2011-07-12$ & 31 & 872 & 13 & 341 \\
$2011-07-13$ & 44 & 1530 & 318 & 36 \\
$2011-07-14$ & 27 & 909 & 0 & 0 \\
$2011-07-15$ & 33 & 1243 & 4 & 05 \\
$2011-07-16$ & 42 & 1216 & 432 & 2120 \\
Total & 216 & 7454 & & 0 \\
\hline
\end{tabular}

Another way to compare the results of different selected subsets is to examine the UC schedules directly rather than their evaluation against the whole set of scenarios. Upon concatenating the unit commitment vectors over the days in $\mathscr{D}$, we have $\mathscr{T}=\{1,2, \cdots, 168\}$. Equation (4.27) computes the optimal committed capacity given scenario set $\mathscr{S}^{\prime} \subseteq \mathscr{S}$.

$$
\phi_{t}\left(\mathscr{S}^{\prime}\right)=\sum_{g \in \mathscr{G}} v_{g t}^{\prime} \bar{P}_{g}, \forall t \in \mathscr{T}
$$

The amount of capacity committed in each hour summarizes the schedule. Relative differences of $\phi_{t}\left(\mathscr{S}_{F S R C: T C}^{\prime}\right)$ and $\phi_{t}\left(\mathscr{S}_{F S R C: T C+P C}^{\prime}\right)$ to $\phi_{t}(\mathscr{S})$ through the week are displayed in Fig. 4.13. In most hours, both FSRC variants selecting half of the total scenarios provide similar amounts of committed capacity as the optimal schedule.

The quality of scenarios selected by FSRC is further investigated by running rolling horizon SRUC on smaller subsets of selected scenarios. Fig. 4.14 displays the performances of selected subsets $\mathscr{S}_{F S R C: T C}^{\prime}$ and $\mathscr{S}_{F S R C: T C+P C}^{\prime}$ over days in $\mathscr{D}$ according to average hourly absolute relative difference in 


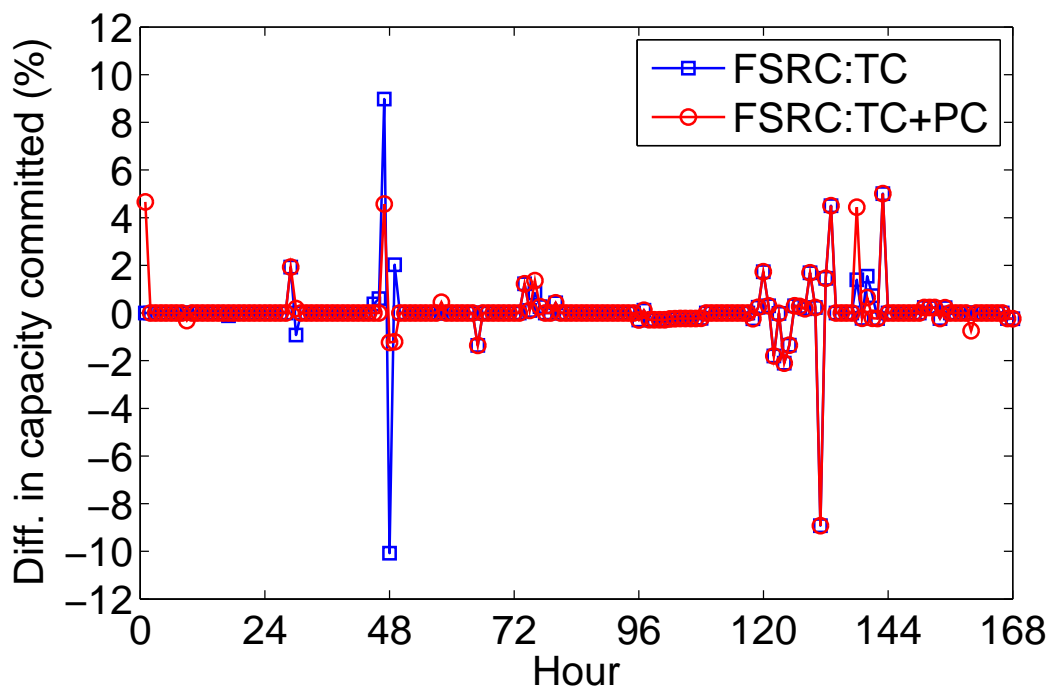

Figure 4.13 Relative differences in hourly capacity committed between half-scenarios based rolling horizon SRUC and all-scenarios based rolling horizon SRUC in week 2011-07-10 to 2011-07-16

committed capacity:

$$
\mathscr{Z}\left(\mathscr{S}^{\prime}\right)=\frac{1}{|\mathscr{T}|} \sum_{t \in \mathscr{T}} \frac{\left|\phi_{t}\left(\mathscr{S}^{\prime}\right)-\phi_{t}(\mathscr{S})\right|}{\phi_{t}(\mathscr{S})}, \mathscr{S}^{\prime} \subset \mathscr{S}
$$

Figure 4.15 compares expected shortage, $\Phi^{+}\left(\mathscr{S} \mid \mathscr{S}^{\prime}\right)$, and excess, $\Phi^{-}\left(\mathscr{S} \mid \mathscr{S}^{\prime}\right)$ for subsets $\mathscr{S}_{\text {FSRC:TC }}^{\prime}$ and $\mathscr{S}_{F S R C: T C+P C}^{\prime}$ to their counterparts from set $\mathscr{S}$, according to measurements (4.29) and (4.30). Either variant of FSRC provides similar shortage and excess to those from the whole scenario set, if the cardinality $n \geq 20$.

$$
\begin{aligned}
\mathscr{W}^{+}\left(\mathscr{S} \mid \mathscr{S}^{\prime}\right) & =\frac{\Psi^{+}\left(\mathscr{S} \mid \mathscr{S}^{\prime}\right)-\Psi^{+}(\mathscr{S} \mid \mathscr{S})}{\Psi^{+}(\mathscr{S} \mid \mathscr{S})}, \mathscr{S}^{\prime} \subset \mathscr{S} \\
\mathscr{W}^{-}\left(\mathscr{S} \mid \mathscr{S}^{\prime}\right) & =\frac{\Psi^{-}\left(\mathscr{S} \mid \mathscr{S}^{\prime}\right)-\Psi^{-}(\mathscr{S} \mid \mathscr{S})}{\Psi^{-}(\mathscr{S} \mid \mathscr{S})}, \mathscr{S}^{\prime} \subset \mathscr{S}
\end{aligned}
$$

Finally, different pairs of penalty settings on shortage and excess are set to conduct sensitivity investigations on customized FSRC for rolling horizon SRUC. Fig. 4.16 shows that the optimal average hourly committed capacity from the subset of scenarios selected by FSRC is close to that from the whole set of scenarios, and subplots in Fig. 4.17 show that $\Psi^{+}\left(\mathscr{S} \mid \mathscr{S}_{F S R C}^{\prime}\right)$ and $\Psi^{-}\left(\mathscr{S} \mid \mathscr{S}_{F S R C}^{\prime}\right)$ are not very sensitive to particular values of the penalty factors. 


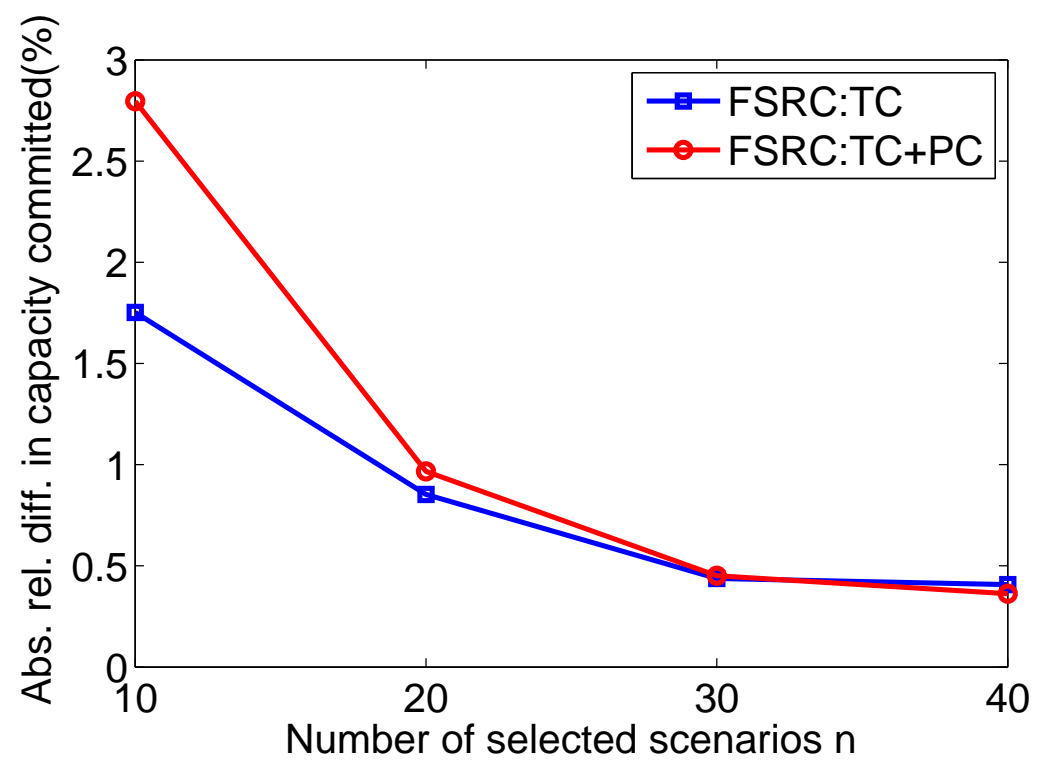

Figure $4.14 \mathscr{Z}\left(\mathscr{S}_{F S R C: T C}^{\prime}\right)$ vs. $\mathscr{Z}\left(\mathscr{S}_{F S R C: T C+P C}^{\prime}\right)$ for different cardinality $n$ in week $2011-07-10$ to 2011-07-16

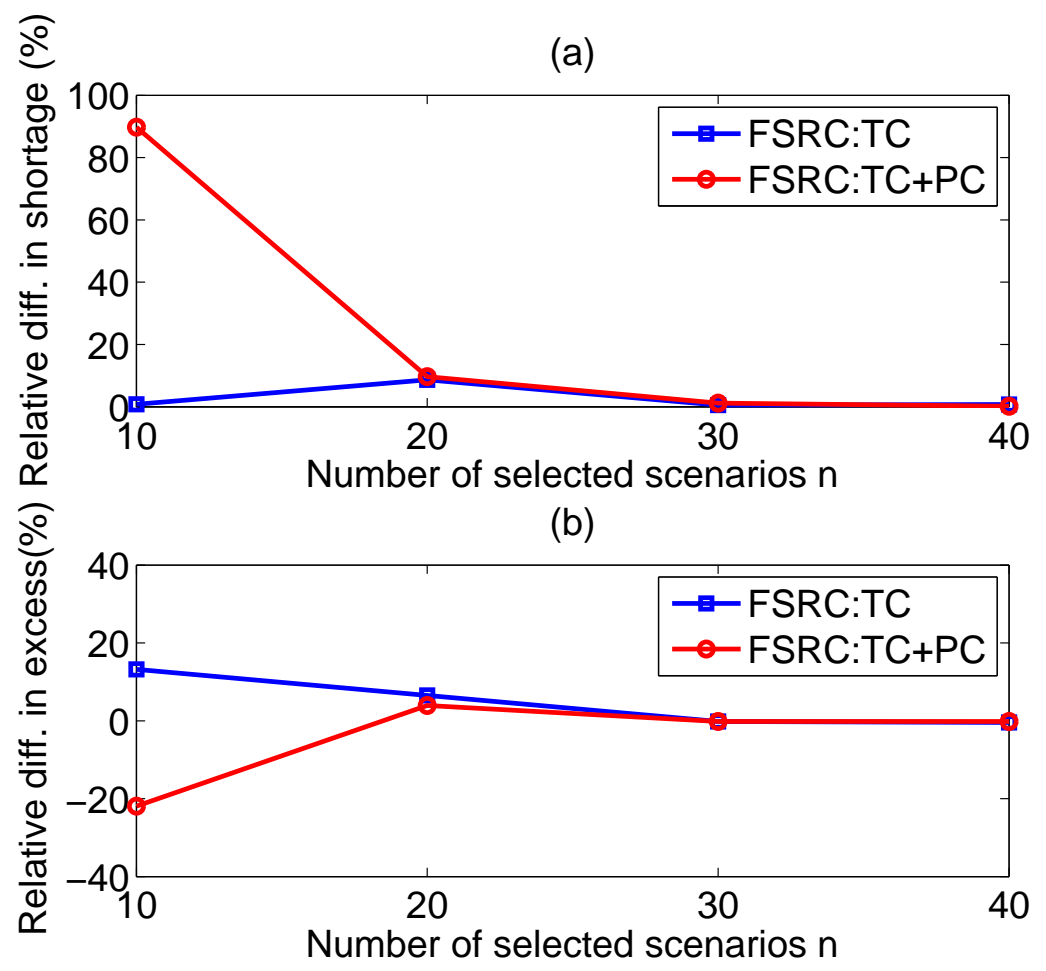

Figure 4.15 Relative differences in expected load imbalance for different cardinalities, $n$, through the week 2011-07-10 to 2011-07-16: (a) $\mathscr{W}^{+}\left(\mathscr{S} \mid \mathscr{S}_{F S R C: T C}^{\prime}\right)$ vs. $\mathscr{W}^{+}\left(\mathscr{S} \mid \mathscr{S}_{F S R C: T C+P C}^{\prime}\right)$, (b) $\mathscr{W}^{-}\left(\mathscr{S} \mid \mathscr{S}_{F S R C: T C}^{\prime}\right)$ vs. $\mathscr{W}^{-}\left(\mathscr{S} \mid \mathscr{S}_{F S R C: T C+P C}^{\prime}\right)$ 


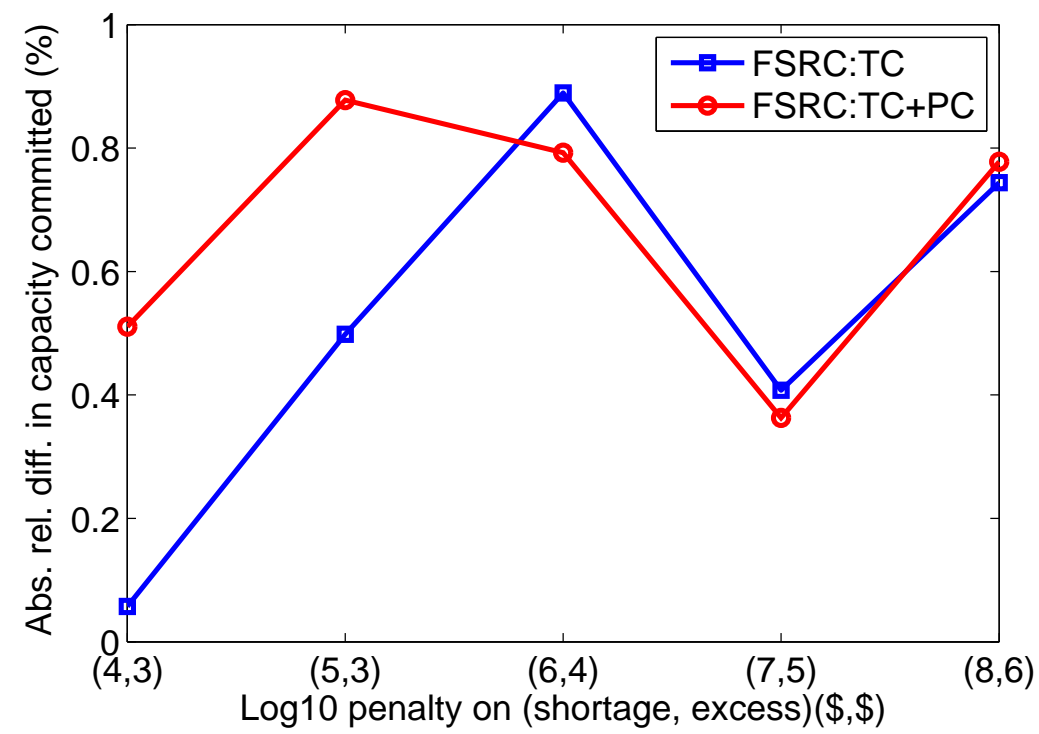

Figure $4.16 \mathscr{Z}\left(\mathscr{S}_{F S R C: T C}^{\prime}\right)$ vs. $\mathscr{Z}\left(\mathscr{S}_{F S R C: T C+P C}^{\prime}\right)$ over different penalty settings in week 2011-07-10 to 2011-07-16
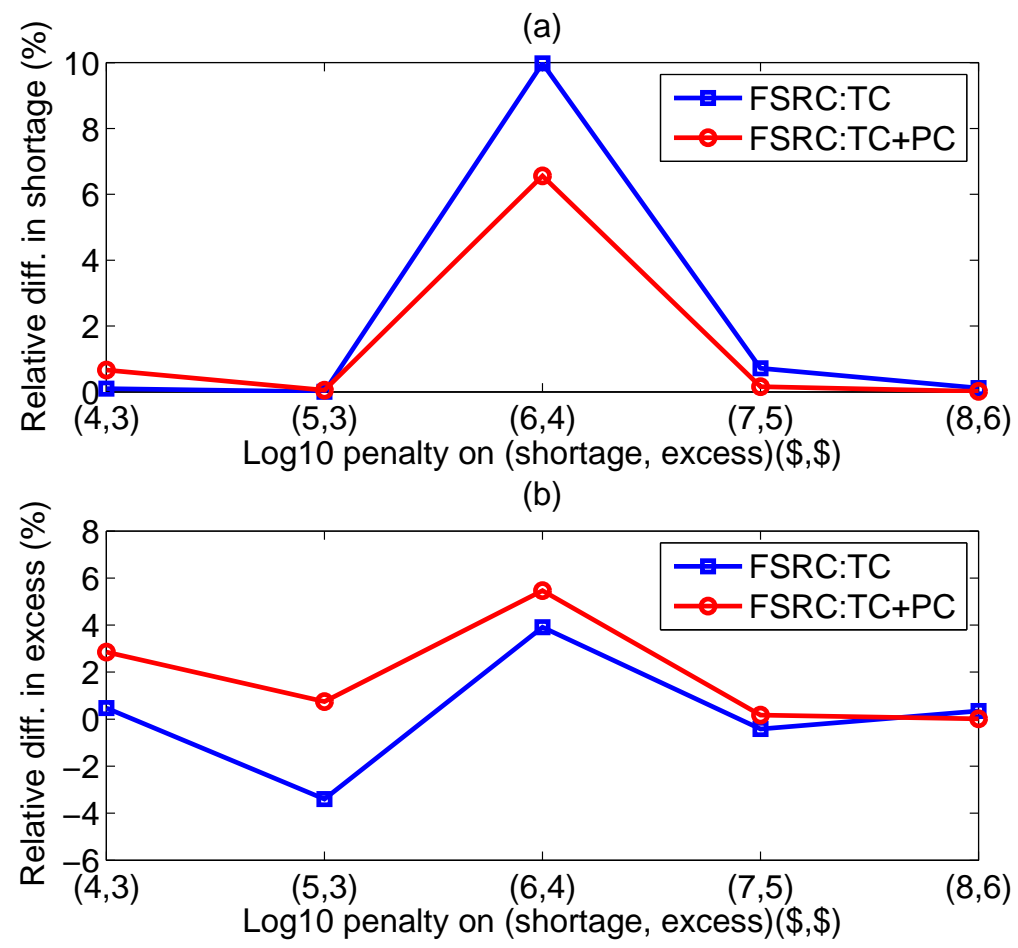

Figure 4.17 Relative differences in load imbalances through different penalty settings:
(a) $\mathscr{W}^{+}\left(\mathscr{S} \mid \mathscr{S}_{F S R C: T C}^{\prime}\right)$ vs. $\mathscr{W}^{+}\left(\mathscr{S} \mid \mathscr{S}_{F S R C: T C+P C}^{\prime}\right)$
(b) $\mathscr{W}^{-}\left(\mathscr{S} \mid \mathscr{S}_{F S R C: T C}^{\prime}\right)$ vs. $\mathscr{W}^{-}\left(\mathscr{S} \mid \mathscr{S}_{F S R C: T C+P C}^{\prime}\right)$ 


\subsection{Conclusion}

In this paper, a scenario reduction method based on solution sensitivity and its customizations for stochastic unit commitment are presented. Numerical investigations on FSRC through single-day and rolling horizon SRUC are performed for a sample of days in a case study distilled from data for an independent system operator in the U.S.. Compared to the classical scenario reduction method, FFS, the customized FSRC tracks aspects on which the decision maker focuses, and thereby leads to more reliable unit commitment schedules. In a rolling horizon study, UC schedules obtained with small subsets of scenarios selected by the customized FSRC methods are similar to those found by optimizing against the whole set of scenarios. A variant that pre-categorizes scenarios based on their qualitative impacts on solutions yields similar results with less computation time for selection. The method uses somewhat artificial penalties on load imbalances but the results are not very sensitive to the particular penalty values used. Tests in this paper were performed on the extensive forms of the two-stage stochastic program, but FSRC could also be used in conjunction with decomposition methods for more efficient solution.

FSRC can be extended easily to a stochastic unit commitment in which variable generation is considered as dispatchable resource. All variable resource generators in that case are considered as elements of $\mathscr{G}$, and viewed as "always on" units through the schedule horizon by fixing corresponding $v_{g t}=1$. The same scenario evaluation procedure, summarization process and cluster technique can be applied to select representative scenarios. We expect that in this case, the excess amounts $\alpha_{b t s}^{-}$are likely to be much smaller overall in the UC evaluation because the ability to curtail variable generation will reduce the impact of underestimating wind power on the day ahead.

The proposed scenario reduction method, FSRC, can be further extended to multi-stage scenario trees by accounting for the nested distance when selecting a representative scenario from each cluster. Similar to the version for the two-stage stochastic unit commitment, a feasible UC strategy will be applied to evaluate scenarios, and solution sensitivity indices will be derived from decisions at each

stage. To avoid overly complicated solution sensitivity indices as the number of stages increase, further research is required to identify efficient ways to summarize multi-stage solution sensitivity. 


\subsection{Remark: Extensions of FSRC}

The above sections in this chapter carefully investigates the proposed scenario reduction method, but does not cover extreme scenarios selection in the scenario reduction procedure. Because the extreme scenarios (one leads to highest load curtail, and the other leads to highest wind energy curtail), may be needed to assess power systems operations in the worst cases, FSRC is extended to include those two extreme scenarios. These extreme scenarios could be inferred in the Summarize procedure. Solution sensitivity indices, $\mathscr{F}_{1}\left(\hat{x}, y_{s}^{*}\right)$ and $\mathscr{F}_{2}\left(\hat{x}, y_{s}^{*}\right)$, the cumulative generation cost and cumulative shortage reveal the scenario with extreme high demand and low wind energy. Similarly, $\mathscr{F}_{1}\left(\hat{x}, y_{s}^{*}\right)$ and $\mathscr{F}_{3}\left(\hat{x}, y_{s}^{*}\right)$, the cumulative generation cost and cumulative excess identify the extreme scenario with extreme low demand and high wind energy. The proposed FSRC in Section 4.4 is then applied to select $n-2$ scenarios from the rest of the scenarios.

We notice that several thermal units are not set to be on/off in several periods in the beginning of planning horizon when higher/lower generation are needed. Therefore, shortage in demand or redundant generation could be resulted because of restrictions on minimum up/down time through the planning horizon. To alleviate the impacts of initial settings on evaluating the quality of FSRC, all thermal units are assumed to be on/off for sufficient time periods at the beginning of the planning horizon. Further investigations on the extended FSRC are performed to assess the power of FSRC on tracking decision makers' concern, the reliability of commitment strategy, when wind energy is considered as dispatchable resource.

Like Section 4.5, the extended FSRC were investigated on a batch of days consisting of three random days from each season. Penetration of wind energy into the power systems are set to no less than $50 \%$ of the available wind energy for each scenario. The same group of 50 generators used in Section 4.5.2 are applied to investigate the extended FSRC with dispatchable wind energy. The commitment costs from the subset of scenarios selected from extended FSRC are compared against the optimal commitment cost in Fig. 4.18. The relative difference is evaluated as following:

$$
\frac{\mathscr{U}\left(\mathscr{S}^{\prime}\right)-\mathscr{U}(\mathscr{S})}{\mathscr{U}(\mathscr{S})} \times 100 \% .
$$

Expected generation costs from the subset of scenarios are compared to the optimal generation cost, as well, displayed in Fig. 4.19. 


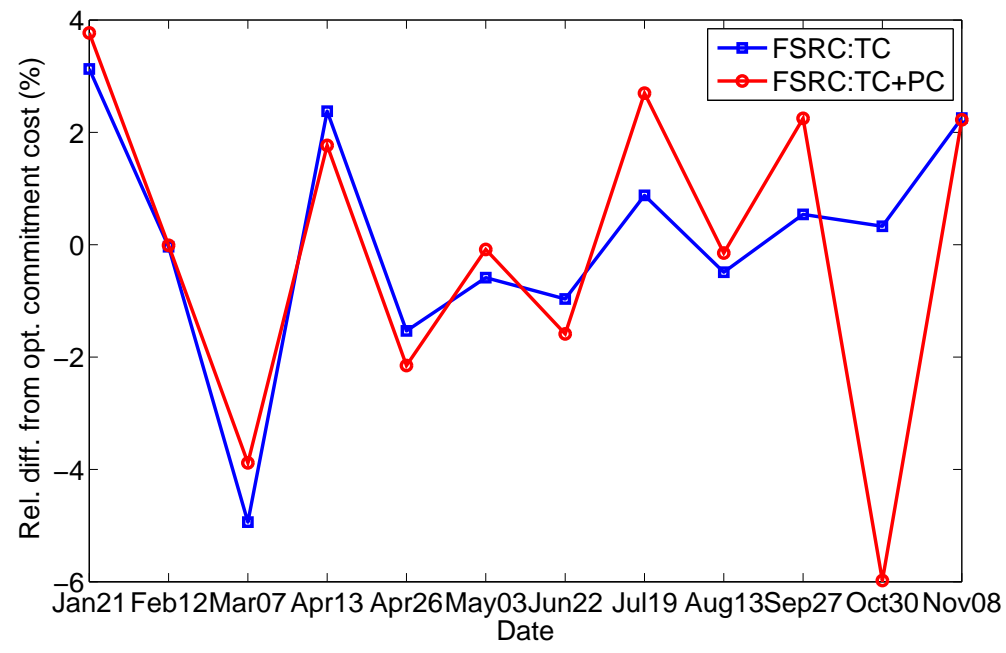

Figure 4.18 Relative differences from the optimal commitment cost

Like formula (4.31), the relative difference from the optimal generation cost is defined below:

$$
\frac{\Phi\left(\mathscr{S} \mid \mathscr{S}^{\prime}\right)-\Phi(\mathscr{S} \mid \mathscr{S})}{\Phi(\mathscr{S} \mid \mathscr{S})} \times 100 \%
$$

According to figures 4.18 and 4.19 , deviations from the optimal commitment cost complement those from the optimal dispatch cost. Therefore, it is reasonable to anticipate that there should not be significant differences between total commitment and generation cost, $\mathscr{U}\left(\mathscr{S}^{\prime}\right)+\Phi\left(\mathscr{S} \mid \mathscr{S}^{\prime}\right)$, and the optimal one, $\mathscr{U}(\mathscr{S})+\Phi(\mathscr{S} \mid \mathscr{S})$, throughout the selected days (Fig.4.20).

As we have mentioned before, expected shortage and excess of a commitment strategy could be other important metric to measure if a scenario reduction method accounts for the generation reliability of power systems. The expected shortage levels against to the whole set of scenarios from the extended FSRC are displayed in Fig. 4.21, compared to the optimal ones. Expected excess derived from extended FSRC and against the whole set of scenarios are provided in Fig. 4.22. Allowance of dispatchable wind energy, (at least 50\% penetration), decreases the redundant variable generation in this case. 


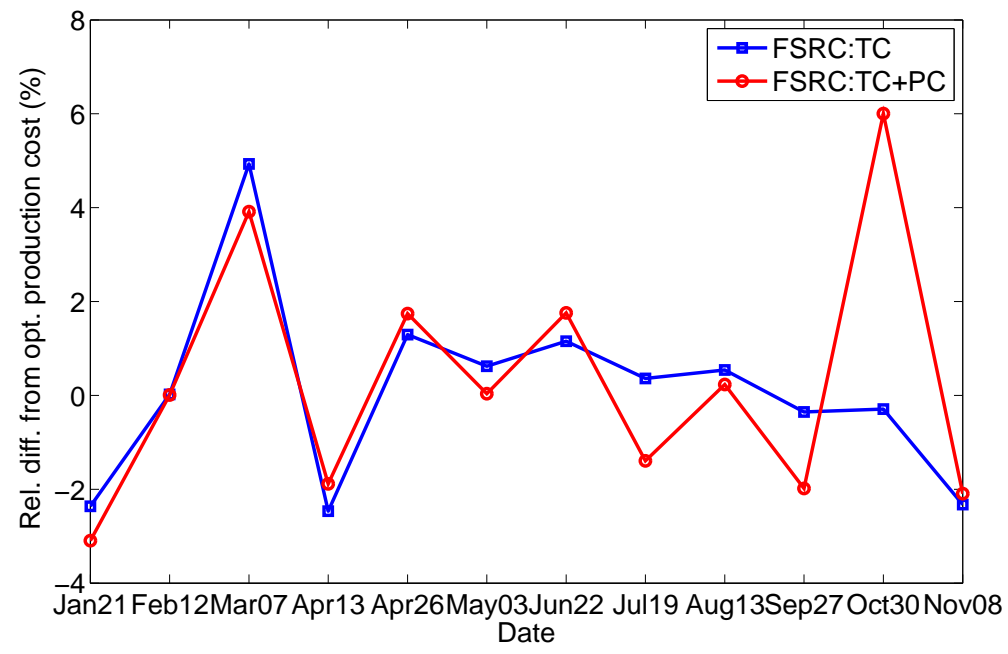

Figure 4.19 Relative differences from the optimal expected generation cost

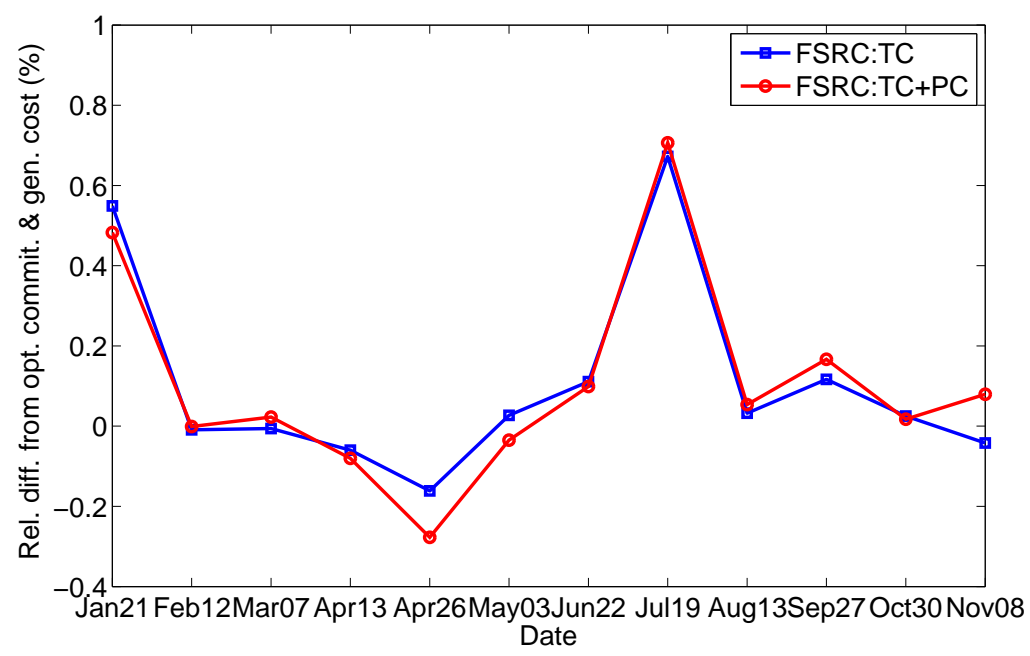

Figure 4.20 Relative differences from the optimal commitment and generation cost 


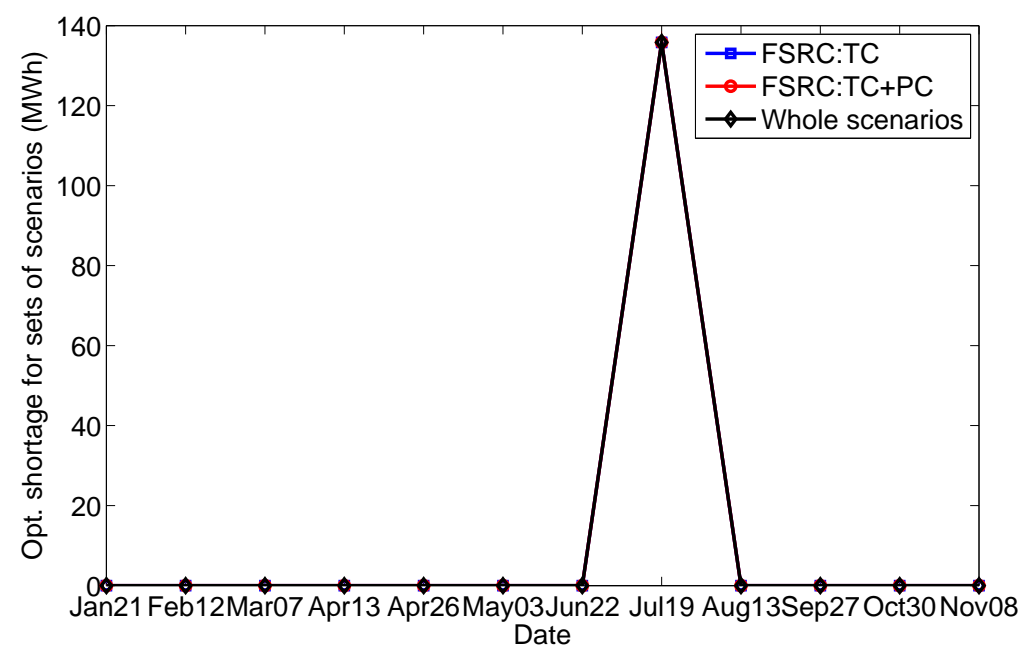

Figure 4.21 Comparisons to the optimal shortage

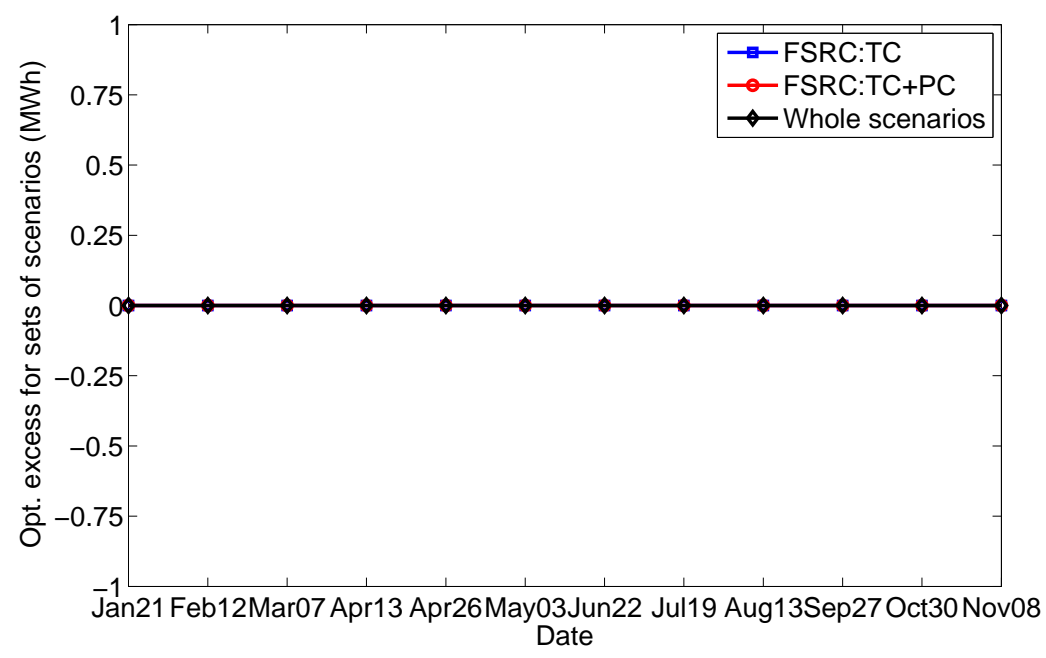

Figure 4.22 Comparisons to the optimal excess 


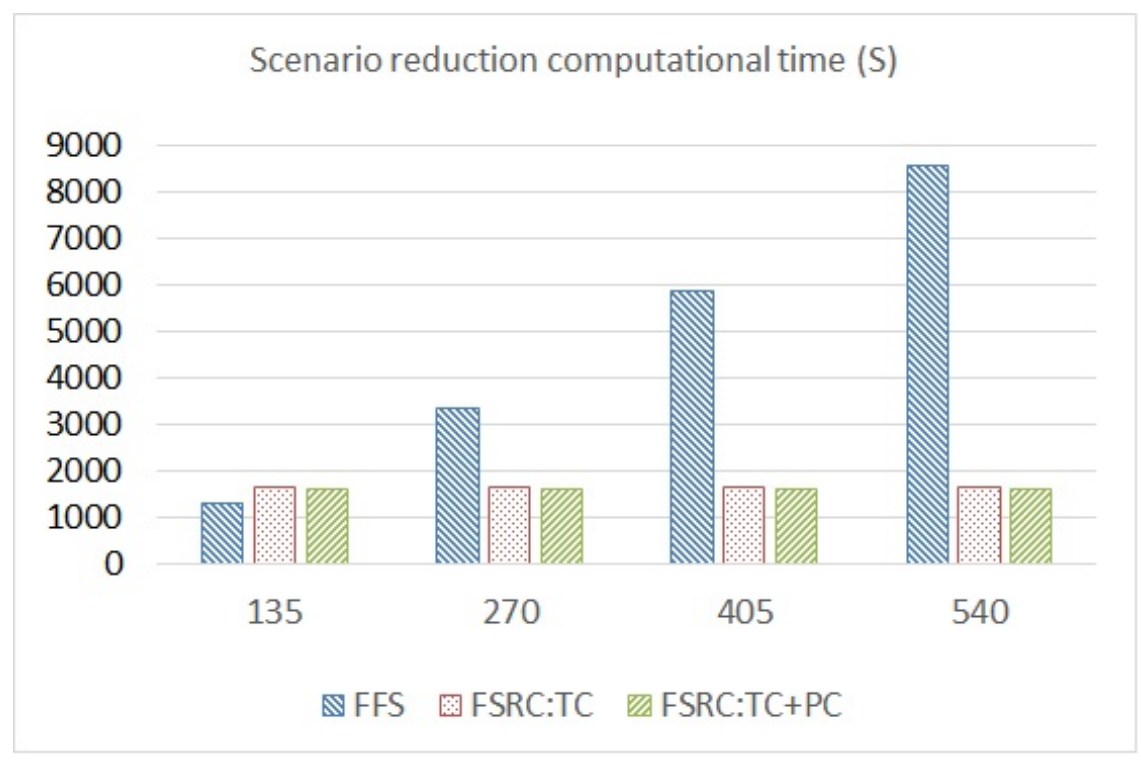

Figure 4.23 Comparisons on total scenario time

Much more pairs of probabilistic scenarios were constructed by generating more load scenarios (27 load scenarios), and then being crossed with 50 wind energy scenarios to investigate if the total computational time of the proposed scenario reduction method, FSRC, would possibly be saved. Fig. 4.23 illustrates that FSRC saves up to $80 \%$ computational time when both the size of the original scenarios and cardinality of selected scenarios are not much small. 


\subsection{Appendix: Concrete stochastic reliability unit commitment model}

\subsubsection{Notation}

\section{Sets and indices:}

$\mathscr{B}$

$\mathscr{G}$

$\mathscr{L}$

$\mathscr{L}_{I}(b)$

$\mathscr{L}_{O}(b)$

$\mathscr{K}_{g}$

$\mathscr{S}$

$\mathscr{T}$

$B F_{\ell}, B T_{\ell}$ set of buses

set of thermal units

set of transmission lines, modeled as directed arcs

set of transmission lines to bus $b$

set of transmission lines from bus $b$

set of time intervals of stairwise start-up cost function

of thermal unit $g$

set of scenarios

set of time periods

buses located at the two ends of transmission line $\ell$, representing the bus injecting power to and absorbing power from line $\ell$, respectively

probability of scenario $s$

ramping-up limit of unit $g(\mathrm{MW} / \mathrm{h})$

ramping-down limit of unit $g(\mathrm{MW} / \mathrm{h})$

start-up ramping limit of unit $g(\mathrm{MW} / \mathrm{h})$

shut-down ramping limit of unit $g(\mathrm{MW} / \mathrm{h})$

capacity of unit $g$ (MW)

minimum power output of unit $g(\mathrm{MW})$

negative susceptance of line $\ell \in \mathscr{L}$

net load at bus $b$ in period $t$ in scenario $s$ (MWh)

maximum capacity of transmission line $\ell$ (MW)

time of unit $g$ has been on, or off at the beginning of scheduling

startup cost of thermal unit $g$ for time intervals $q \in$ $\mathscr{K}_{g}(\$)$

reserve requirement in period $t(\mathrm{MWh})$

minimum up and down times of unit $g$

penalties on load imbalance ( $\$ / \mathrm{MWh}$ )

penalties on reserve requirement imbalance $(\$ / M W h)$

number of blocks of the piecewise linear generation cost function of unit $g$ 

$\delta_{j g t s} \quad$ energy generated in block $j$ of the piecewise linear generation cost function of unit $g$ in period $t$ in sce- nario $s(\mathrm{MWh})$
$\lambda_{j g} \quad$ slope of block $j$ of the piecewise linear generation cost function of unit $g(\$ / M W h)$
$\gamma_{j g} \quad$ upper limit of block $j$ of the piecewise linear genera- tion cost function (MWh)
no-load cost of unit $g(\$)$
commitment cost function of unit $g$ in period $t(\$)$
generation cost function of unit $g$ in period $t$ in sce- nario $s(\$)$

\section{Decision variables:}

$v_{g t} \in\{0,1\}$

$p_{\text {gts }} \geq 0$

$\bar{p}_{g t s} \geq 0$

$\alpha_{b t s}^{+}, \alpha_{b t s}^{-} \geq 0$

$\beta_{t s}^{+}, \beta_{t s}^{-} \geq 0$

$\theta_{b t s}$

$\omega_{\ell t s}$ first-stage decision, binary variable, equal to 1 if unit $g$ is on in period $t$, and 0 otherwise generation level of unit $g$ in period $t$ in scenario $s$ for $g \in \mathscr{G}$ (MW)

maximum available power generation for unit $g$ in period $t$ in scenario $s$ for $g \in \mathscr{G}$ (MW)

auxiliary variables, representing shortage and excess in load supply at bus $b$ in period $t$ in scenario $s$, respectively (MWh)

ancillary variables, representing shortage and excess in reserve requirement in period $t$ in scenario $s$ (MWh)

phase angle at bus $b$ in period $t$ in scenario $s$ (radians) line power of transmission line $\ell$ in period $t$ in scenarios $s$, unrestricted in sign because power can flow in either directions on a line

\subsubsection{Mathematical model}

The full concrete formulation of SRUC extends the deterministic UC model in (Carrión and Arroyo, 2006) to a two-stage stochastic program. The commitments of thermal units are considered as first-stage decisions. Second-stage decision variables include generation level of each unit, and corresponding maximum available generation level. The following presents a two-stage stochastic program by viewing each hour as a period.

\subsubsection{Objective function}

$$
\min \sum_{t \in \mathscr{T}} \sum_{g \in \mathscr{G}} c_{g t}^{u}\left(v_{g t}\right)+\sum_{s \in \mathscr{S}} \xi_{s} \zeta_{s}
$$


The objective function (4.33) consists of two parts: the cost related to commitments of units, like startup, shutdown and no-load costs; and the cost related to generation and penalties on load and reserve requirement imbalances, upon realization of a scenario in the second stage, as shown in (4.34).

$$
\zeta_{s}=\sum_{t \in \mathscr{T}} \sum_{g \in \mathscr{G}} c_{g t s}^{p}\left(p_{g t s}\right)+\sum_{t \in \mathscr{T}} \sum_{b \in \mathscr{B}}\left(\Gamma_{\alpha}^{+} \alpha_{b t s}^{+}+\Gamma_{\alpha}^{-} \alpha_{b t s}^{-}\right)+\sum_{t \in \mathscr{T}}\left(\Gamma_{\beta}^{+} \beta_{t s}^{+}+\Gamma_{\beta}^{-} \beta_{t s}^{-}\right)
$$

The goal of SRUC is to minimize total commitment cost, expected generation cost and expected penalties on imbalances in generation and reserve. The following section presents operational constraints.

\subsubsection{Constraints}

\section{Energy balance at each bus:}

$$
\begin{aligned}
\sum_{g \in \mathscr{G}(b)} p_{g t s} & +\sum_{\ell \in \mathscr{L}_{I}(b)} \omega_{\ell t s}-\sum_{\ell \in \mathscr{L}_{O}(b)} \omega_{\ell t s} \\
& +\alpha_{b t s}^{+}-\alpha_{b t s}^{-}=d_{b t s}, \forall b \in \mathscr{B}, \forall t \in \mathscr{T}, \forall s \in \mathscr{S}
\end{aligned}
$$

Formula (4.35) describes the relationship between load demand and generated and net transmitted energy for each bus.

\section{Reserve requirements:}

$$
\sum_{g \in \mathscr{G}} \bar{p}_{g t s}+\beta_{t s}^{+}-\beta_{t s}^{-}=\sum_{b \in \mathscr{B}} d_{b t s}+R_{t}, \forall t \in \mathscr{T}, \forall s \in \mathscr{S}
$$

Reserve requirements (4.36) maintain reliability if contingencies that are not modeled in scenarios occur; e.g., outages of generators or transmission lines.

\section{Line Power:}

$$
\begin{aligned}
& \omega_{\ell t s}=B_{\ell}\left(\theta_{B F_{\ell} t s}-\theta_{B T_{\ell} t s}\right), \forall t \in \mathscr{T}, \forall \ell \in \mathscr{L}, \forall s \in \mathscr{S} \\
& -F_{\ell} \leq \omega_{\ell t s} \leq F_{\ell}, \forall t \in \mathscr{T}, \forall \ell \in \mathscr{L}, \forall s \in \mathscr{S}
\end{aligned}
$$

Power flow in each transmission line is formulated and restricted in (4.37) and (4.38). Formulation (4.37) is a linear, lossless DC approximation of the relationship between phase angles and power flow on a transmission line. 


\section{Generation limits:}

$$
\underline{P}_{g} v_{g t} \leq p_{g t s} \leq \bar{p}_{g t s} \leq \bar{P}_{g} v_{g t}, \forall g \in \mathscr{G}, \forall t \in \mathscr{T}, \forall s \in \mathscr{S}
$$

Available power generation level of a thermal unit depends on its operational status, as in (4.39). The difference between maximum available generation level $\bar{p}_{g t s}$ and actual generation level $p_{g t s}$ indicates the contribution of unit $g$ to the reserve requirement in period $t$ in scenario $s$. In addition, the maximum available generation level of a thermal unit in a period is coupled by possible generation levels in preceding and succeeding periods.

\section{Ramp rate limits:}

$$
\begin{aligned}
\bar{p}_{g t s} & \leq p_{g, t-1, s}+\Delta_{g}^{r u} v_{g, t-1}+\Delta_{g}^{s u}\left(v_{g t}-v_{g, t-1}\right) \\
& +\bar{P}_{g}\left(1-v_{g t}\right), \forall g \in \mathscr{G}, \forall t \in \mathscr{T}, \forall s \in \mathscr{S} \\
\bar{p}_{g t s} & \leq \bar{P}_{g} v_{g, t+1} \\
& +\Delta_{g}^{s d}\left(v_{g t}-v_{g, t+1}\right), \forall g \in \mathscr{G}, \forall t=1, \cdots,|\mathscr{T}|-1 \\
p_{g, t-1, s}-p_{g t s} & \leq \Delta_{g}^{r d} v_{g t}+\Delta_{g}^{s d}\left(v_{g, t-1}-v_{g t}\right) \\
& +\bar{P}_{g}\left(1-v_{g, t-1}\right), \forall g \in \mathscr{G}, \forall t \in \mathscr{T}, \forall s \in \mathscr{S}
\end{aligned}
$$

Formulas (4.40) - (4.42) represent maximum available changes in generation levels of each unit between two consecutive periods.

\section{Minimum up-time constraints:}

$$
\begin{aligned}
& \sum_{t=1}^{H_{g}^{+}}\left(1-v_{g t}\right)=0, \forall g \in \mathscr{G} \\
& \sum_{i=t}^{t+T_{g}^{U}-1} v_{g i} \geq\left(v_{g t}-v_{g, t-1}\right), \forall g \in \mathscr{G}, \forall t=H_{g}^{+}+1, \cdots,|\mathscr{T}|-T_{g}^{U}+1 \\
& \sum_{i=t}^{|\mathscr{T}|}\left(v_{g i}-v_{g t}+v_{g, t-1}\right) \geq 0, \forall g \in \mathscr{G}, \forall t=|\mathscr{T}|-T_{g}^{U}+2, \cdots,|\mathscr{T}|
\end{aligned}
$$




\section{Minimum down-time constraints:}

$$
\begin{aligned}
& \sum_{t=1}^{H_{g}^{-}} v_{g t}=1, \forall g \in \mathscr{G} \\
& \sum_{i=t}^{t+T_{g}^{D}-1}\left(1-v_{g t}\right) \geq\left(v_{g, t-1}-v_{g t}\right), \forall g \in \mathscr{G}, \forall t=H_{g}^{-}+1, \cdots,|\mathscr{T}|-T_{g}^{D}+1 \\
& \sum_{i=t}^{|\mathscr{T}|}\left(1-v_{g i}-v_{g, t-1}+v_{g t}\right) \geq 0, \forall g \in \mathscr{G}, \forall t=|\mathscr{T}|-T_{g}^{D}+2, \cdots,|\mathscr{T}|
\end{aligned}
$$

Thermal units cannot be shut down (started up) immediately after being started up (shut down), because these operations can only be performed under gradual change of temperature, which translates to time periods. Formulas (4.43) - (4.48) use binary variables to describe these restrictions on thermal units.

\section{Commitment cost:}

$$
c_{g t}^{u}\left(v_{g t}\right) \geq \rho_{g k}\left(v_{g t}-\sum_{i=1}^{\min (t-1, k)} v_{g, t-i}\right)+a_{g} v_{g t}, \forall g \in \mathscr{G}, \forall t \in \mathscr{T}, \forall k \in \mathscr{K}_{g}
$$

The start-up cost function is monotonically increasing along the time of a thermal unit has been

off. Stairwise start-up cost function is adopted and formulated in the first term of (4.49), where $\mathscr{K}_{g}=$ $\left\{k_{1}, \cdots, k_{\mathscr{N}_{g}}\right\}$, and $\rho_{g k_{i}} \leq \rho_{g k_{i+1}}, i=1, \cdots, \mathscr{N}_{g}-1$. Notice that, $\rho_{g k}$ includes shutdown cost in the case study. The second term is no-load cost of unit $g$ which will occur once a unit is committed.

\section{Generation cost function:}

$$
\begin{aligned}
& c_{g t s}^{p}\left(p_{g t s}\right)=\sum_{j=1}^{J} \lambda_{j g} \delta_{j g t s}, \forall g \in \mathscr{G}, \forall t \in \mathscr{T}, \forall s \in \mathscr{S} \\
& p_{g t s}=\underline{P}_{g}+\sum_{j=1}^{J} \delta_{j g t s}, \forall g \in \mathscr{G}, \forall t \in \mathscr{T}, \forall s \in \mathscr{S} \\
& \delta_{1 g t s} \leq \gamma_{1 j}-\underline{P}_{g}, \forall g \in \mathscr{G}, \forall t \in \mathscr{T}, \forall s \in \mathscr{S} \\
& \delta_{j g t s} \leq \gamma_{j g}-\gamma_{j-1, g}, \forall g \in \mathscr{G}, \forall t \in \mathscr{T}, \forall s \in \mathscr{S}, \forall j=2, \cdots, J-1 \\
& \delta_{J g t s} \leq \bar{P}_{g}-\gamma_{J-1, g}, \forall g \in \mathscr{G}, \forall t \in \mathscr{T}, \forall s \in \mathscr{S} \\
& \delta_{j g t s} \geq 0, \forall g \in \mathscr{G}, \forall t \in \mathscr{T}, \forall s \in \mathscr{S}, \forall j=1, \cdots, J
\end{aligned}
$$

Equations (4.50)-(4.55) compute piecewise-linear generation costs. 
A mixed integer linear program (MILP) extensive form of SRUC has been formulated in (4.33) (4.58), with boundary defined for each decision variable, as follows.

\subsubsection{Bounds}

$$
\begin{aligned}
& 0 \leq p_{g t s} \leq \bar{P}_{g}, \forall g \in \mathscr{G}, \forall t \in \mathscr{T}, \forall s \in \mathscr{S} \\
& 0 \leq \bar{p}_{g t s} \leq \bar{P}_{g}, \forall g \in \mathscr{G}, \forall t \in \mathscr{T}, \forall s \in \mathscr{S} \\
& -\pi \leq \theta_{b t s} \leq \pi, \forall b \in \mathscr{B}, \forall t \in \mathscr{T}, \forall s \in \mathscr{S}
\end{aligned}
$$

This model can be easily extended to the situation in which variable generation, such as wind, is viewed as dispatchable resource. Set $\mathscr{G}$ will include variable energy generators, and $d_{b t s}$ will represent load rather than net load. In formula (4.39), we can set $v_{g t}$ to 1 for any variable energy generator $g$, and allow $\bar{P}_{g}$ to vary by scenario. Then other constraints will be suitable for variable energy generators. 


\section{CHAPTER 5. GENERAL CONCLUSIONS}

This dissertation is a combination of three papers to provide rounded insights addressing several issues of two-stage stochastic programs for long-term generation expansion planning and short-term generation planning of power systems with uncertain resources from the perspective of the system operators.

From the perspective of a centralized power market, the first paper, presented in Chapter 2, identifies a long-term expansion strategy of several generation technologies by minimizing total expected cost of a two-stage stochastic program. Future possible evolutions of annual load and natural gas price through the planning horizon are depicted by probabilistic scenario trees, which are generated by first verifying the fit of geometric Brownian motion processes to historical data and then by matching statistical moments of annual load and natural gas price, as well as their correlations. A heuristic scenario reduction method, FSWC, is developed to reduce the nearly 60,000 joint annual load and natural gas prices scenarios to a modest number of scenarios which allow solution of the corresponding stochastic mixed integer program in a manageable amount of computational time. In addition to the substantially improved computational efficiency of the scenario reduction procedure, FSWC keeps similar solutions as those from FFS when modest-sized subsets of scenarios are selected. In a hypothetical power system with six types of generators, FSWC leads to very similar expansion strategies, expected generation levels and expected costs with different cardinalities of subsets, but only takes up to $15 \%$ as much time for reduction and solution combined as FFS (Table 2.13). Therefore, FSWC seems to be appropriate to select a modest number of scenarios from a huge set of scenarios for a stochastic long-term generation expansion planning problem.

The first paper simplifies the generation expansion planning model by temporarily ignoring the topology of power systems. However, network constraints significantly increase the computational complexity. If generation expansion planning and transmission expansion planning are performed si- 
multaneously, some efforts need to be taken to identify the key first-stage decisions and accurately group scenarios by efficiently evaluating the impacts of scenarios on the decisions. In addition, the two-stage stochastic programming formulation of generation expansion planning in the first paper could be further extended to a rolling horizon decision procedure over the whole planning horizon by updating annual load and natural gas price scenarios periodically.

Tractable solution times for long term planning problems are desirable because they permit exploration of the sensitivity to parameter settings not explicitly modeled as probabilistic scenarios. Efficient computation for short-term planning is not only desirable but required. The remaining two papers focus on the computational issues of stochastic short-term generation planning, because time constraints imposed by daily market operations require that it be solved quickly.

The second paper, presented in Chapter 3, develops a day-ahead hourly load model, FWEp, based on weather forecasts in order to account for the effects from weather forecast errors on load forecasting. First, segmentation and clustering are proposed to filter seasonal calendar effects, as well as keeping a consistent relationship between load and weather conditions in each segment. A method to transform load data from different day types to a reference day type is applied to enrich the data set used for model-fitting. Finally, FWEp captures the non-weather dependent load pattern in each season, and then fits the relationship between load and weather conditions by a family of twice differentiable functions. In an ISO-wide case study, the flexible fitting procedure not only improves the accuracy of day-ahead load predictions, but also preserves the similarity of intraday hourly serial correlations to those from actual load values. This modeling fidelity implies that FWEp would be helpful to generate practical probabilistic load scenarios through a day. Because the paper only focuses on short-term load modeling, one future work can cover ways to generate load scenarios from the predictions of FWEp. The prerequisite is to estimate hourly prediction errors through a day, as in (Singham et al., 2013); then a sampling approach or an approximation approach based on conditional expectations as in (Rios et al., 2013) may need to be investigated to select prediction errors while maintaining the intraday hourly serial correlations. Another possible direction is to extend the epi-spline based fitting procedure to variable generation, i.e. wind energy and solar energy. Because the trajectory of wind or solar energy through a day would be more volatile than that of hourly load, the traditional linear regression method may lack sufficient flexibility to capture the pattern of wind or solar energy. In addition, some data transformation 
techniques might be investigated first for the variable generation before the fitting procedure.

The contributions of the last paper, presented in Chapter 4, include the development and customization of a heuristic scenario reduction method, FSRC, which not only considers probabilities and distances among scenarios, but also follows the decision maker's major concerns about reliability and cost. Instead of evaluating influence from scenarios on decisions by solving a mixed integer program (MIP) for each scenario, FSRC only requires solving a MIP for a single deterministic expected-value problem, and the resulted commitment strategy is applied to evaluate generation levels, shortage (load curtailment) and excess (wind energy curtailment) by solving a continuous linear program for each scenario so that the similarities among scenarios can be revealed. With the help of pre-categorizing scenarios before clustering, the computational complexity of FSRC is further alleviated. In downsampled instances from an ISO, we find that more reliable commitment of units, from the perspective of less shortage and lower scheduling cost, can be obtained by optimizing against a subset of scenarios selected by FSRC rather than by FFS. In addition, numerical results for a rolling horizon SRUC show that FSRC keeps the solution time manageable while maintaining solution quality even when the reduction of the scenario set is substantial. Although tests of FSRC were performed by solving the extensive forms of two-stage stochastic programs for unit commitment, it could be directly combined with decomposition techniques to improve the computational efficiency.

The dissertation discusses problem-dependent scenario reduction methods, FSWC and FSRC, for two-stage stochastic mixed integer programs (Feng and Ryan, 2013, 2014a,b). Customizations of these two methods to reveal the influences of scenarios on decisions are crucial for the scenario reduction process. However, FSRC should be more computationally efficient than FSWC when the problem has difficult constraints, e.g. unit commitment problem, because FSRC only solves MIP once and one sequence of LPs, but FSWC solves MIP for every scenario. According to the computational time comparisons provided in Section 4.7, if there are a lot of scenarios and the computational complexity of the problem is less than that of the unit commitment problem, FSRC provides quicker scenario reduction procedure than FFS in general. Although FSRC is designed for two-stage stochastic programs, it could be extended to multi-stage scenario trees by using nested distance (Pflug and Pichler, 2012; Timonina, 2013). Similar to the two-stage version, a given unit commitment would be employed to measure the relative differences among scenarios. However, an efficient way needs to be investigated to avoid overly 
complicated solution sensitivity indices as the number of stages increases.

Moreover, scenario reduction techniques should not be restricted for the purpose of obtaining suboptimal solutions for a two-stage stochastic program within manageable time. Indeed, scenario reduction could also be applied to find good feasible initial points for several decomposition techniques, e.g. progressive hedging algorithm (Rockafellar and Wets, 1991; Watson and Woodruff, 2011) and column generation algorithm (Shiina and Birge, 2004). As an example, a two-stage stochastic program could be solved for a reduced subset of scenarios (e.g. $20 \%$ or $30 \%$ of the whole set of scenarios) to get a suboptimal and feasible first-stage decision, and then the obtained feasible first-stage decision would be input into the progressive hedging algorithm as an initial solution. The total number of iterations of progressive hedging algorithm before convergence is expected to be significantly decreased. For the column generation algorithm, several subsets of scenarios need to be selected first, and a sequence of suboptimal and feasible solutions could be obtained by solving the two-stage stochastic program on these subsets. The sequence of suboptimal and feasible solutions will form the initial restricted set of the column generation algorithm. Once these feasible solutions are identified from crucial subsets of scenarios, the computational time of column generation based approach would be significantly decreased. 


\section{BIBLIOGRAPHY}

3TIER Inc. (2012). Private communication. http://www.3tier.com/en/.

Amjady, N. (2001). Short-term hourly load forecasting using time-series modeling with peak load estimation capability. IEEE Transactions on Power Systems, 16(3):498-505.

Anton, H. (1999). Calculus: A New Horizon. Wiley, New York.

Beltratti, A., Consiglio, A., and Zenios, S. A. (1999). Scenario modeling for the management ofinternational bond portfolios. Annals of Operations Research, 85:227-247.

Bertocchi, M., Moriggia, V., and Dupačová, J. (2000). Sensitivity of bond portfolio's behavior with respect to random movements in yield curve: A simulation study. Annals of Operations Research, 99(1-4):267-286.

Bertsimas, D., Litvinov, E., Sun, X. A., Zhao, J., and Zheng, T. (2013). Adaptive robust optimization for the security constrained unit commitment problem. IEEE Transactions on Power Systems, 28(1):5263.

Black, J. D. (2011). Load hindcasting: A retrospective regional load prediction method using reanalysis weather data. Master's thesis, University of Massachusetts Amherst.

Bloom, J. A. (1982). Long-range generation planning using decomposition and probabilistic simulation. IEEE Transactions on Power Apparatus and Systems, (4):797-802.

Botterud, A., Ilic, M. D., and Wangensteen, I. (2005). Optimal investments in power generation under centralized and decentralized decision making. IEEE Transactions on Power Systems, 20(1):254263. 
Botterud, A., Zhou, Z., Wang, J., Valenzuela, J., Sumaili, J., Bessa, R. J., Keko, H., and Miranda, V. (2011). Unit commitment and operating reserves with probabilistic wind power forecasts. In 2011 IEEE Trondheim PowerTech, pages 1-7. IEEE.

Bouffard, F. and Galiana, F. (2008). Stochastic security for operations planning with significant wind power generation. IEEE Transactions on Power Systems, 23(2):306-316.

Bouffard, F., Galiana, F., and Conejo, A. (2005). Market-clearing with stochastic security-part I: Formulation. IEEE Transactions on Power Systems, 20(4):1818-1826.

Carino, D., Myers, D., and Ziemba, W. (1998). Concepts, technical issues, and uses of the russellyasuda kasai financial planning model. Operations Research, 46(4):450-462.

Carøe, C. and Schultz, R. (1998). A two-stage stochastic program for unit commitment under uncertainty in a hydro-thermal power system. Technical report, Konrad-Zuse-Zentrum für Informationstechnik, Berlin.

Carpentier, P., Gohen, G., Culioli, J.-C., and Renaud, A. (1996). Stochastic optimization of unit commitment: A new decomposition framework. IEEE Transactions on Power Systems, 11(2):1067-1073.

Carrión, M. and Arroyo, J. M. (2006). A computationally efficient mixed-integer linear formulation for the thermal unit commitment problem. IEEE Transactions on Power Systems, 21(3):1371-1378.

Charlton, N. and Singleton, C. (2014). A refined parametric model for short term load forecasting. International Journal of Forecasting, 30(2):364-368.

Charytoniuk, W., Chen, M.-S., and Van Olinda, P. (1998). Nonparametric regression based short-term load forecasting. IEEE Transactions on Power Systems, 13(3):725-730.

Che, J. and Wang, J. (2014). Short-term load forecasting using a kernel-based support vector regression combination model. Applied Energy, 132:602-609.

Chen, B., Wang, J., Wang, L., He, Y., and Wang, Z. (2014). Robust optimization for transmission expansion planning: Minimax cost vs. minimax regret. IEEE Transactions on Power Systems, 29(6):3069_ 3077. 
Chen, Y., Luh, P. B., Guan, C., Zhao, Y., Michel, L. D., Coolbeth, M. A., Friedland, P. B., and Rourke, S. J. (2010). Short-term load forecasting: Similar day-based wavelet neural networks. IEEE Transactions on Power Systems, 25(1):322-330.

Cheung, K., Gade, D., Monroy, C. S., Ryan, S. M., Watson, J.-P., Wets, R. J.-B., and Woodruff, D. L. (2014). Toward scalable stochastic unit commitment - Part 2: Assessing solver performance. Energy Systems, Forthcoming.

Chuang, A. S., Wu, F., and Varaiya, P. (2001). A game-theoretic model for generation expansion planning: Problem formulation and numerical comparisons. IEEE Transactions on Power Systems, 16(4):885-891.

Constantinescu, E., Zavala, V., Rocklin, M., Lee, S., and Anitescu, M. (2011). A computational framework for uncertainty quantification and stochastic optimization in unit commitment with wind power generation. IEEE Transactions on Power Systems, 26(1):431-441.

Corbus, D., King, J., Mousseau, T., Zavadil, R., Heath, B., Hecker, L., Lawhorn, J., Osborn, D., Smit, J., and Hunt, R. (2011). Eastern wind integration and transmission study. http://www. nrel. gov/docs/fy09osti/46505.pdf.

Dantzig, G. B., Glynn, P. W., Avriel, M., Stone, J. C., Entriken, R., and Nakayama, M. (1989). Decomposition techniques for multi-area generation and transmission planning under uncertainty. Technical report, Electric Power Research Institution, Palo Alto, CA (USA).

Dapklus, W. D. and Bowe, T. R. (1984). Planning for new electric generation technologies: A stochastic dynamic programming approach. IEEE Transactions on Power Apparatus and Systems, (6):14471453.

Dempster, M. and Thompson, R. (1999). EPVI-based importance sampling solution proceduresfor multistage stochastic linear programmes on parallel MIMD architectures. Annals of Operations Research, 90:161-184.

Dupačová, J., Consigli, G., and Wallace, S. W. (2000). Scenarios for multistage stochastic programs. Annals of Operations Research, 100(1):25-53. 
Dupačová, J., Gröwe-Kuska, N., and Römisch, W. (2003). Scenario reduction in stochastic programming: An approach using probability metrics. Mathematical Programming, 95(3):493-511.

Engle, R. F., Mustafa, C., and Rice, J. (1992). Modelling peak electricity demand. Journal of Forecasting, 11(3):241-251.

European Federation of Energy Traders (2013). EU renewable energy policy. http://www.euractiv.com/energy/eu-renewable-energy-policy-linksdossier-188269.

Fan, S. and Chen, L. (2006). Short-term load forecasting based on an adaptive hybrid method. IEEE Transactions on Power Systems, 21(1):392-401.

Fan, S., Chen, L., and Lee, W.-J. (2009). Short-term load forecasting using comprehensive combination based on multimeteorological information. IEEE Transactions on Industry Applications, 45(4):14601466.

Fan, S. and Hyndman, R. J. (2012). Short-term load forecasting based on a semi-parametric additive model. IEEE Transactions on Power Systems, 27(1):134-141.

Feinberg, E. A. and Genethliou, D. (2005). Load forecasting. In Applied Mathematics for Restructured Electric Power Systems, pages 269-285. Springer.

Feng, Y., Gade, D., Ryan, S. M., Watson, J.-P., Wets, R. J.-B., and Woodruff, D. L. (2013). A new approximation method for generating day-ahead load scenarios. In 2013 IEEE Power and Energy Society General Meeting, pages 1-5. IEEE.

Feng, Y., Rios, I., Ryan, S. M., Spürkel, K., Watson, J.-P., Wets, R. J.-B., and Woodruff, D. L. (2014). Toward scalable stochastic unit commitment - Part 1: Load scenario generation. Energy Systems, Forthcoming.

Feng, Y. and Ryan, S. M. (2012). Application of scenario reduction to LDC and risk based generation expansion planning. In 2012 IEEE Power and Energy Society General Meeting, pages 1-8. IEEE.

Feng, Y. and Ryan, S. M. (2013). Scenario construction and reduction applied to stochastic power generation expansion planning. Computers \& Operations Research, 40(1):9-23. 
Feng, Y. and Ryan, S. M. (2014a). Scenario reduction for stochastic unit commitment with wind penetration. In 2014 IEEE Power and Energy Society General Meeting, pages 1-5. IEEE.

Feng, Y. and Ryan, S. M. (2014b). Solution sensitivity-based scenario reduction for stochastic unit commitment. Computational Management Science, pages 1-34. DOI: 10.1007/s10287-014-0220-z.

Fleten, S.-E. and Pettersen, E. (2005). Constructing bidding curves for a price-taking retailer in the Norwegian electricity market. IEEE Transactions on Power Systems, 20(2):701-708.

Fleten, S.-E. and Wallace, S. W. (2009). Delta-hedging a hydropower plant using stochastic programming. In Optimization in the Energy Industry, pages 507-524. Springer.

Gonen, T. (1988). Modern Power System Analysis. John Wiley \& Sons, New York.

Gorenstin, B. G., Campodónico, N. M., da Costa, J. P., and Pereira, M. V. F. (1993). Power system expansion planning under uncertainty. IEEE Transactions on Power Systems, 8(1):129-136.

Growe-Kuska, N., Heitsch, H., and Romisch, W. (2003). Scenario reduction and scenario tree construction for power management problems. In IEEE Bologna Power Tech Conference Proceedings. IEEE.

Guan, Y. and Wang, J. (2014). Uncertainty sets for robust unit commitment. IEEE Transactions on Power Systems, 29(3):1439-1440.

Hagan, M. T. and Behr, S. M. (1987). The time series approach to short term load forecasting. IEEE Transactions on Power Systems, 2(3):785-791.

Haida, T. and Muto, S. (1994). Regression based peak load forecasting using a transformation technique. IEEE Transactions on Power Systems, 9(4):1788-1794.

Heitsch, H. and Römisch, W. (2003). Scenario reduction algorithms in stochastic programming. Computational Optimization and Applications, 24(2):187-206.

Heitsch, H. and Römisch, W. (2007). A note on scenario reduction for two-stage stochastic programs. Operations Research Letters, 35(6):731-738. 
Heitsch, H. and Römisch, W. (2009a). Scenario tree modeling for multistage stochastic programs. Mathematical Programming, 118(2):371-406.

Heitsch, H. and Römisch, W. (2009b). Scenario tree reduction for multistage stochastic programs. Computational Management Science, 6(2):117-133.

Heitsch, H. and Römisch, W. (2011). Stability and scenario trees for multistage stochastic programs. In Stochastic Programming, volume 150 of International Series in Operations Research \& Management Science, pages 139-164. Springer.

Heitsch, H., Römisch, W., and Strugarek, C. (2006). Stability of multistage stochastic programs. SIAM Journal on Optimization, 17(2):511-525.

Henrion, R., Küchler, C., and Römisch, W. (2008). Discrepancy distances and scenario reduction in two-stage stochastic mixed-integer programming. Journal of Industrial and Management Optimization, 4(2):363-384.

Henrion, R., Küchler, C., and Römisch, W. (2009). Scenario reduction in stochastic programming with respect to discrepancy distances. Computational Optimization and Applications, 43(1):67-93.

Hong, T. (2011). Short term electric load forecasting. PhD thesis, North Carolina State University.

Hong, T., Wang, P., and Willis, H. L. (2011). A naïve multiple linear regression benchmark for short term load forecasting. In 2011 IEEE Power and Energy Society General Meeting, pages 1-6. IEEE.

Høyland, K., Kaut, M., and Wallace, S. W. (2003). A heuristic for moment-matching scenario generation. Computational Optimization and Applications, 24(2):169-185.

Høyland, K. and Wallace, S. W. (2001). Generating scenario trees for multistage decision problems. Management Science, 47(2):295-307.

Huang, S.-J. and Shih, K.-R. (2003). Short-term load forecasting via ARMA model identification including non-Gaussian process considerations. IEEE Transactions on Power Systems, 18(2):673679. 
ISO New England (2013a). Create demand forecast. http://www.isone.com/rules_proceds/operating/sysop/out_sched/sop_outsch_0040_0010.pdf.

ISO New England (2013b). Hourly zonal information. http://www.isone.com/markets/hstdata/znl_info/hourly/index.html.

Jaccard, M. (2004). Renewable portfolio standard. http://www.emrg.sfu.ca/media/publications /2004/RPSEncyclopedia.pdf.

JCSP (2012). Joint coordinated system plan $08 . \quad$ https://www.midwestiso.org/Library/Repository/Study/JCSP/JCSP_Report_Volume_1.pdf.

Jiang, R., Wang, J., and Guan, Y. (2012). Robust unit commitment with wind power and pumped storage hydro. IEEE Transactions on Power Systems, 27(2):800-810.

Jin, S. and Ryan, S. M. (2011). Capacity expansion in the integrated supply network for an electricity market. IEEE Transactions on Power Systems, 26(4):2275-2284.

Jin, S., Ryan, S. M., Watson, J., and Woodruff, D. L. (2011). Modeling and solving a large-scale generation expansion planning problem under uncertainty. Energy Systems, 2(3-4):209-242.

Kagiannas, A. G., Askounis, D. T., and Psarras, J. (2004). Power generation planning: A survey from monopoly to competition. International Journal of Electrical Power \& Energy Systems, 26(6):413421.

Kall, P. and Wallace, S. W. (1994). Stochastic Programming. John Wiley \& Sons, New York.

Kaut, M. and Wallace, S. W. (2007). Evaluation of scenario-generation methods for stochastic programming. Pacific Journal of Optimization, 3(2):257-271.

Kleywegt, A. J., Shapiro, A., and Homem-de Mello, T. (2002). The sample average approximation method for stochastic discrete optimization. SIAM Journal on Optimization, 12(2):479-502.

Kouwenberg, R. (2001). Scenario generation and stochastic programming models for asset liability management. European Journal of Operational Research, 134(2):279-292. 
Küchler, C. and Vigerske, S. (2007). Decomposition of multistage stochastic programs with recombining scenario trees. Stochastic Programming E-Print Series (SPEPS).

Kyriakides, E. and Polycarpou, M. (2007). Short term electric load forecasting: A tutorial. In Trends in Neural Computation, pages 391-418. Springer.

Latorre, J. M., Cerisola, S., and Ramos, A. (2007). Clustering algorithms for scenario tree generation: Application to natural hydro inflows. European Journal of Operational Research, 181(3):1339-1353.

Liu, J. M., Chen, R., Liu, L.-M., and Harris, J. L. (2006). A semi-parametric time series approach in modeling hourly electricity loads. Journal of Forecasting, 25(8):537-559.

Liu, Y., Ferris, M., and Zhao, F. (2014). Computational study of security constrained economic dispatch with multi-stage rescheduling. IEEE Transactions on Power Systems, pages 1-10. DOI: 10.1109/TPWRS.2014.2336667.

Lurie, P. and Goldberg, M. (1998). An approximate method for sampling correlated random variables from partially-specified distributions. Management Science, 44(2):203-218.

Mahoney, W., Parks, K., Wiener, G., Liu, Y., Myers, W., Sun, J., Delle Monache, L., Hopson, T., Johnson, D., and Haupt, S. E. (2011). A wind power forecasting system to optimize grid integration. IEEE Transactions on Sustainable Energy, 3(4):670 - 682.

Mahoney, W. P., Parks, K., Wiener, G., Liu, Y., Myers, W. L., Sun, J., Delle Monache, L., Hopson, T., Johnson, D., and Haupt, S. E. (2012). A wind power forecasting system to optimize grid integration. IEEE Transactions on Sustainable Energy, 3(4):670-682.

Manning, C., Raghavan, P., and Schütze, H. (2008). Introduction to Information Retrieval. Cambridge University Press, Cambridge, 1st edition.

Marathe, R. R. and Ryan, S. M. (2005). On the validity of the Geometric Brownian motion assumption. The Engineering Economist, 50(2):159-192.

Mathworks (2011). R 2011a statistics tool box: Kmeans documentation. http://www.mathworks.com/help/toolbox/stats/kmeans.html. 
Mo, B., Hegge, J., and Wangensteen, I. (1991). Stochastic generation expansion planning by means of stochastic dynamic programming. IEEE Transactions on Power Systems, 6(2):662-668.

Mohandes, M. (2002). Support vector machines for short-term electrical load forecasting. International Journal of Energy Research, 26(4):335-345.

Moral-Carcedo, J. and Vicens-Otero, J. (2005). Modelling the non-linear response of spanish electricity demand to temperature variations. Energy Economics, 27(3):477-494.

Morales, J., Conejo, A. J., Liu, K., and Zhong, J. (2012). Pricing electricity in pools with wind producers. IEEE Transactions on Power Systems, 27(3):1366-1376.

Morales, J. M., Pineda, S., Conejo, A. J., and Carrion, M. (2009). Scenario reduction for futures market trading in electricity markets. IEEE Transactions on Power Systems, 24(2):878-888.

Nowak, M. and Römisch, W. (2000). Stochastic Lagrangian relaxation applied to power scheduling in a hydro-thermal system under uncertainty. Annals of Operations Research, 100(1-4):251-272.

Papalexopoulos, A. D. and Hesterberg, T. C. (1990). A regression-based approach to short-term system load forecasting. IEEE Transactions on Power Systems, 5(4):1535-1547.

Papavasiliou, A. and Oren, S. (2013). Multiarea stochastic unit commitment for high wind penetration in a transmission constrained network. Operations Research, 61(3):578-592.

Papavasiliou, A., Oren, S., and O'Neill, R. (2011). Reserve requirements for wind power integration: A scenario-based stochastic programming framework. IEEE Transactions on Power Systems, 26(4):2197-2206.

Park, J., Park, Y., Won, J., and Lee, K. Y. (2000). An improved genetic algorithm for generation expansion planning. IEEE Transactions on Power Systems, 15(3):916-922.

Park, Y., Won, J., Park, J., and Kim, D. (1999). Generation expansion planning based on an advanced evolutionary programming. IEEE Transactions on Power Systems, 14(1):299-305.

Pflug, G. C. (2001). Scenario tree generation for multiperiod financial optimization by optimal discretization. Mathematical Programming, 89(2):251-271. 
Pflug, G. C. and Pichler, A. (2012). A distance for multistage stochastic optimization models. SIAM Journal on Optimization, 22(1):1-23.

Philpott, A., Craddock, M., and Waterer, H. (2000). Hydro-electric unit commitment subject to uncertain demand. European Journal of Operational Research, 125(2):410-424.

Rios, I., Wets, R. J.-B., and Woodruff, D. L. (2013). Multi-period forecasting and scenario generation with limited data. Technical report, University of California Davis, Davis CA 95616.

Rockafellar, R. T. and Wets, R. J.-B. (1991). Scenarios and policy aggregation in optimization under uncertainty. Mathematics of operations research, 16(1):119-147.

Roh, J. H., Shahidehpour, M., and Fu, Y. (2007). Market-based coordination of transmission and generation capacity planning. IEEE Transactions on Power Systems, 22(4):1406-1419.

Römisch, W. and Vigerske, S. (2010). Recent progress in two-stage mixed-integer stochastic programming with applications to power production planning. In Handbook of Power Systems I, pages 177-208. Springer.

Ross, S. M. (2010). Brownian motion and stationary process. In Introduction to Probability Models, pages 636-637. Academic Press, New York, 10th edition.

Royset, J. O. and Wets, R. J.-B. (2014). From data to assessments and decisions: Epi-spline technology. In Tutorials in Operations Research: Bridging Data and Decision, pages 27-53. INFORMS.

Ruiz, P. A., Philbrick, C. R., Zak, E., Cheung, K. W., and Sauer, P. W. (2009). Uncertainty management in the unit commitment problem. IEEE Transactions on Power Systems, 24(2):642-651.

Sandia National Laboratories (2013a). Pyomo. https://software.sandia.gov/trac/coopr/wiki/Pyomo.

Sandia National Laboratories (2013b). PySP. https://software.sandia.gov/trac/coopr/wiki/PySP.

Shiina, T. and Birge, J. R. (2004). Stochastic unit commitment problem. International Transactions in Operational Research, 11(1):19-32.

Sigman, K. (2006). Geometric Brownian motion. http://www.columbia.edu/ks20/FE-Notes/4700-07Notes-GBM.pdfS. 
Singham, D. I., Royset, J. O., and Wets, R. J.-B. (2013). Density estimation of simulation output using exponential epi-splines. In 2013 Winter Simulation Conference, pages 755-765. IEEE.

Smith, J. E. (1993). Moment methods for decision analysis. Management Science, 39(3):340-358.

Takriti, S., Birge, J. R., and Long, E. (1996). A stochastic model for the unit commitment problem. IEEE Transactions on Power Systems, 11(3):1497-1508.

Takriti, S., Krasenbrink, B., and Wu, L. S.-Y. (2000). Incorporating fuel constraints and electricity spot prices into the stochastic unit commitment problem. Operations Research, 48(2):268-280.

Taylor, J. W. (2003). Short-term electricity demand forecasting using double seasonal exponential smoothing. Journal of the Operational Research Society, 54(8):799-805.

Taylor, J. W. (2010). Triple seasonal methods for short-term electricity demand forecasting. European Journal of Operational Research, 204(1):139-152.

Tidball, R., Bluestein, J., Rodriguez, N., and Knoke, S. (2010). Cost and performance assumptions for modeling electricity generation technologies. http://www.nrel.gov/docs/fy11osti/48595.pdf.

Timonina, A. V. (2013). Multi-stage stochastic optimization: The distance between stochastic scenario processes. Computational Management Science, pages 1-25. DOI: 10.1007/s10287-013-0185-3.

Tuohy, A., Meibom, P., Denny, E., and O'Malley, M. (2009). Unit commitment for systems with significant wind penetration. IEEE Transactions on Power Systems, 24(2):592-601.

U.S. Energy Information Administration (2008). Annual electric generator report. http://www.eia.doe.gov/cneaf/electricity/page/capacity/ existingunitsbs2008.xls.

U.S. Energy Information Administration (2010a). Annual energy outlook 2010 with projections to 2035. ftp://ftp.eia.doe.gov/forecast ing/0554(2010).pdf.

U.S. Energy Information Administration (2010b). Annual energy review: electricity overview. http://www.eia.doe.gov/totalenergy/data/monthly/index.cfm\#electricity.

U.S. Energy Information Administration (2010c). Coal prices in selected years. http://www.eia.gov/totalenergy/data/annual/pdf/sec7_19.pdf. 
U.S. Energy Information Administration (2010d). Electric power annual 2009. http://www.eia.gov/cneaf/electricity/epa/epa.pdf.

U.S. Energy Information Administration (2010e). Electric power industry 2009: Year in review. http://www.eia.gov/cneaf/electricity/epa/epa_sum.html.

U.S. Energy Information Administration (2011). Natural gas data. http://www.eia.doe.gov/dnav/ng/hist/n9190us3a.htm.

U.S. Energy Information Administration (2012a). AEO 2012 early release report. http://www.eia.gov/forecasts/aeo/er/.

U.S. Energy Information Administration (2012b). Use of electricity. http://www.eia.gov/energyexplained/index.cfm?page=electricity_use.

U.S. Energy Information Administration (2013a). Annual Energy Outlook 2013 with Projections to 2040. http://www.eia.gov/forecasts/aeo/pdf/0383(2013).pdf.

U.S. Energy Information Administration (2013b). Electricity end use. http://www.eia.gov/beta/MER/index.cfm?tbl=T07.06\#/?f=M.

U.S. Energy Information Administration (2013c). How much of our electricity is generated from renewable energy? http://www.eia.gov/energy_in_brief/article/renewable_electricity.cfm.

Wang, J., Shahidehpour, M., and Li, Z. (2008). Security-constrained unit commitment with volatile wind power generation. IEEE Transactions on Power Systems, 23(3):1319-1327.

Wang, Y. (2010). Scenario reduction heuristics for a rolling stochastic programming simulation of bulk energy flows with uncertain fuel costs. PhD thesis, Iowa State University, Ames, IA.

Wang, Y., Xia, Q., and Kang, C. (2011). Secondary forecasting based on deviation analysis for shortterm load forecasting. IEEE Transactions on Power Systems, 26(2):500-507.

Watson, J.-P. and Woodruff, D. (2011). Progressive hedging innovations for a class of stochastic mixedinteger resource allocation problems. Computational Management Science, 8(4):355-370. 
Watson, J.-P., Woodruff, D., and Hart, W. (2012). PySP: Modeling and solving stochastic programs in python. Mathematical Programming Computation, 4(2):109-149.

Wets, R. J.-B. and Bianchi, S. W. (2006). Term and volatility structures. In Handbook of Asset and Liability Management: Theory and Methodology, pages 26-28. Elsevier.

Wu, L., Shahidehpour, M., and Li, T. (2007). Stochastic security-constrained unit commitment. IEEE Transactions on Power Systems, 22(2):800-811.

Zheng, Q., Wang, J., Pardalos, P., and Guan, Y. (2013). A decomposition approach to the two-stage stochastic unit commitment problem. Annals of Operations Research, 210(1):387-410. 